


\title{
Aqueous-Phase Reforming Multiphase reaction engineering at the microscale
}

\author{
Renée Ripken
}


The work in this thesis was carried out at the Applied Microfluidics for BioEngineering Research and Mesoscale Chemical Systems departments, at the TechMed Centre and MESA+ Institute for Nanotechnology at the University of Twente, The Netherlands, and at the Guenther Laboratory at the Mechanical and Industrial Engineering department at the University of Toronto, Canada.

This research was financially support by the Netherlands Centre for Multiscale Catalytic Energy Conversion (MCEC), an NWO Gravitation program funded by the Ministry of Education, Culture and Science of the government of The Netherlands.
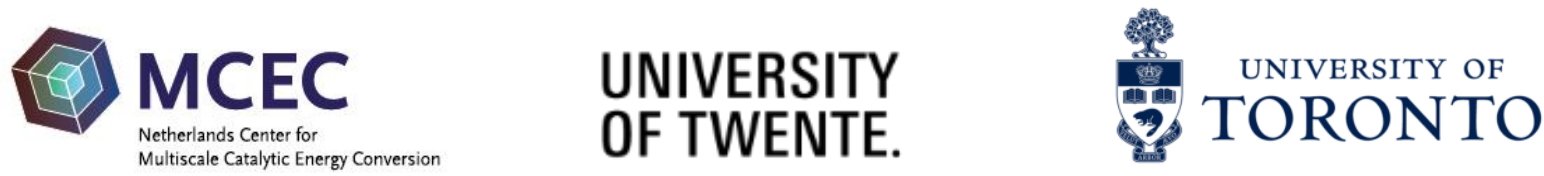

Title: Aqueous-Phase Reforming Multiphase reaction engineering at the microscale Author: Renée Ripken

Cover design: antart/Renée Ripken

Printed by: Ipskamp Printing

ISBN: 978-90-365-4784-0

DOI: $10.3990 / 1.9789036547840$

URL: https://doi.org/10.3990/1.9789036547840

(C) 2019 Enschede, The Netherlands. All rights reserved. No parts of this thesis may be reproduced, stored in a retrieval system or transmitted in any form or by any means without permission of the author.

Alle rechten voorbehouden. Niets uit deze uitgave mag worden vermenigvuldigd, in enige vorm of op enige wijze, zonder voorafgaande schriftelijke toestemming van de auteur. 
This thesis has been approved by:

Promotor:

Prof. Dr. J.G.E. Gardeniers

Co-promotor:

Prof. Dr. Ir. S. Le Gac

\section{Committee members}

Prof. J. N. Kok (chairman)

Prof. Dr. J.G.E. Gardeniers (promotor)

Prof. Dr. Ir. S. Le Gac (co-promotor)

Prof. Dr. Ir. H.J.M. ter Brake

Prof. Dr. R.M. van der Meer

Dr. J.A. Wood

Prof. Dr. Ir. H. J. Heeres

Prof. Dr. Ir. W. de Malsche

Dr. A. Guenther
University of Twente

University of Twente

University of Twente

University of Twente

University of Twente

University of Twente

Rijksuniversiteit Groningen

Vrije Universiteit Brussel

University of Toronto 



\section{AQUEOUS-PHASE REFORMING}

\section{MULTIPHASE REACTION ENGINEERING AT THE MICROSCALE}

\section{PROEFSCHRIFT}

ter verkrijging van

de graad van doctor aan de Universiteit Twente,

op gezag van de rector magnificus,

Prof. Dr. T.T.M. Palstra,

volgens besluit van het College voor Promoties

in het openbaar te verdedigen

op vrijdag 28 juni 2019 om 14:45 uur

door

Renée Maria Ripken

geboren op 14 januari 1991

te Waalwijk, Nederland 



\section{Table of content}

1. General introduction 1

1.1 Background and motivation 2

1.2 Hydrogen production from biomass 5

1.3 Aqueous-Phase Reforming (APR) 9

1.4 Microfluidics as a tool to study and optimize chemical reactions 13

$\begin{array}{lll}1.5 & \text { Multiphase catalytic microreactors } & 15\end{array}$

$\begin{array}{lll}1.6 & \text { Thesis outline } & 17\end{array}$

$\begin{array}{ll}\text { References } & 19\end{array}$

$\begin{array}{ll}\text { 2. Thermodynamics of APR } & 27\end{array}$

$\begin{array}{lll}2.1 & \text { Introduction } & 28\end{array}$

2.2 Materials and methods 29

2.3 Results and discussion $\quad 30$

2.4 Conclusion 38

$\begin{array}{ll}\text { Acknowledgements } & 38\end{array}$

References 38

A2.1 Vapor-liquid equilibrium $\quad 41$

A2.2 Saturated vapor pressure $\quad 45$

A2.3 Equations $\quad 46$

$\begin{array}{ll}\text { A2.4 Data comparison } & 49\end{array}$

3. High-pressure high-temperature microfluidics 5

3.1 Introduction $\quad 52$

3.2 Materials and methods 53

3.3 Results and discussion 56

3.4 Conclusion 63

$\begin{array}{ll}\text { References } & 63\end{array}$ 

bubbles

$\begin{array}{lll}4.1 & \text { Introduction } & 68\end{array}$

4.2 Materials and methods $\quad 69$

$\begin{array}{lll}4.3 & \text { Results and discussion } & 74\end{array}$

4.4 Conclusion $\quad 81$

References $\quad 81$

A4.1 Temperature dependency physical properties 85

A4.2 Reaction data $\quad 86$

\section{Towards controlled bubble nucleation in microreactors for} enhanced mass transport

5.1 Introduction

5.2 Materials and methods $\quad 88$

5.3 Results and discussion $\quad 90$

5.4 Conclusion $\quad 95$

$\begin{array}{ll}\text { Acknowledgements } & 104\end{array}$

$\begin{array}{ll}\text { References } & 105\end{array}$

105

\section{Controlled catalyst deposition}

$\begin{array}{lll}6.1 & \text { Introduction } & 109\end{array}$

6.2 Materials and methods $\quad 110$

6.3 Results and discussion 112

6.4 Conclusion 116

Acknowledgements $\quad 121$

References $\quad 121$

A6.1 SEM analysis of washcoated silicon substrates 122 
7. Fabrication of a gas/liquid microseparator with ultra-low dead volume

7.1 Introduction

7.2 Design and fabrication

7.3 Result and discussion 126

7.4 Conclusion

134

Acknowledgements

138

References

138

A7.1 Process flow

8. Electrochemical study of photocatalytic reforming of biomass

8.1 Introduction

8.2 Materials and methods

8.3 Results and discussion

8.4 Conclusion

Acknowledgements 175

References

\section{Conclusion and outlook}

9.1 Conclusion

9.2 Outlook

References

\section{Samenvatting}

Acknowledgements

About the author 



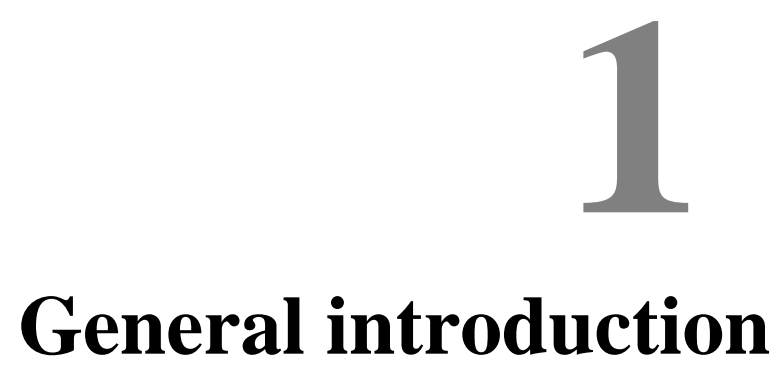

Part of this chapter is adapted from:

Ripken, R.M. et al, The hydrogen economy: can APR contribute? In preparation 


\subsection{Background and motivation}

Continuous burning of fossil fuels, emitting $\mathrm{CO}_{2}$ and other greenhouse gases, are acknowledged to cause many environmental problems, of which global warming is the most prominent. Currently, the earth's atmosphere consists of $408 \mathrm{ppm} \mathrm{CO} 2$ compared to $280 \mathrm{ppm}$ before the industrial revolution, which caused the average global temperature to increase by $1 \mathrm{~K}$ due to human action since $1880 .^{3-5}$ This temperature increase is so severe, that it seriously affects ecosystems and the weather: extreme drought ${ }^{8}$ and/or rainfall ${ }^{9}$ are becoming more common and significantly influence life on earth. For these reasons, the United Nations have now declared climate change as one of the major issues of modern times. ${ }^{10}$

If action is not taken now, the $\mathrm{CO}_{2}$ emission will continue to increase even further. As the world's population is growing, so is also its energy demand. Almost 10 billion people are expected to inhabit earth by 2050 , compared to 7.55 billion in $2017 .{ }^{11}$ Not only will there be more of us, but we will also be living longer; projections predict an average global life expectancy close to 75 years in 2050 compared to 68 years today. ${ }^{11}$ In addition, we are becoming wealthier, so that energy is now affordable for more people. ${ }^{12-13}$ As a result, the US Energy Information Administration predicts that the worldwide energy needs will increase by $28 \%$ from 2015 to $2040 .{ }^{14}$

This intensified energy demand is not without consequences: higher energy consumption at faster rates will further aggravate climate change, with all its devastating effects. Furthermore, our current energy economy is almost exclusively based on fossil fuels, such as petroleum, coal, and natural gas. For instance, $80 \%$ of the energy in the U.S. was generated from such resources in 2017. ${ }^{15}$ If the energy consumption continues to increase at the same rate as it is today, these limited resources will be depleted in 50 to 100 years' time. ${ }^{16}$ As the economic effects of dwindling fossil fuel sources become more pronounced, oil prices will likely increase dramatically.

Politics have now finally picked up on this dire matter, which is reflected by the number of meetings on climate change and energy, such as in Paris in 2015 and its follow-up in Katowice in 2018. The importance to act now is supported by a great number of scientific reports, issued for instance by the Intergovernmental Panel on Climate Change (IPCC), concluding that global warming must be limited to $1.5 \mathrm{~K}$ to avoid severe consequences for both nature and economy. Altogether, these facts demonstrate that the world can no longer rely on fossil fuels only for its 
energy supply, and that there must be a switch to renewable and clean energy alternatives. Although this energy transition is still slow, the use of renewables has increased since 2015, and is predicted to continue to grow to a consumption level close to that of coal by $2040 .{ }^{14}$

\subsubsection{Defining 'renewable'}

However, one could wonder when an energy resource is sustainable, as many definitions exist of the word 'renewable'. In 2014, the International Energy Agency (IEA) defined renewable energy as "Energy derived from natural processes (e.g., sunlight and wind) that are replenished at a faster rate than they are consumed". ${ }^{17}$ Others describe it as "energy produced by wind, sun, and other sources that will never run out" ${ }^{18}$ Even in the scientific literature, the definition differs from publication to publication. In this thesis, an energy resource is considered renewable when consuming the energy source neither leads to depletion, nor forms harmful products during its use that would negatively affect the environment. Importantly, this definition includes evaluation of the production method and/or energy storage, which is currently very often overlooked when classifying energy sources.

Additionally, a clear distinction must be made between an 'energy source' and an 'energy carrier', which is sometimes also called an 'energy vector'. To classify as an energy source, the energy form has to exist in nature, as for example sunlight, wind or tidal energy. In any other case, for instance when the considered energy is generated by light, it must be named an energy carrier. ${ }^{19}$ For example, electricity produced by a solar panel falls in that last category.

Preferably, the energy is used directly from the source, circumventing the cycle of energy harvesting, storage, and transport. However, energy sources are not always readily available and can fluctuate over time: ${ }^{20-21}$ solar energy can only be harvested during the day, and wind is weather-dependent. Hydropower does not have this intermittence problem, but is geographically limited: rapid waters only exist in places with significant height differences and not all countries have coastal shores. Therefore, energy carriers are almost unavoidable, not only to supply energy at any time, but also to transport it to the location where it is needed. 


\subsubsection{Hydrogen as a renewable energy carrier: opportunities and challenges}

Many renewable energy carriers exist, of which hydrogen is a particularly attractive one. Hydrogen, found either as $\mathrm{H}_{2}$ or as part of a molecule, only forms harmless water during combustion. ${ }^{22}$ Its wide flammability limit (4 - $75 \mathrm{vol} \%$, compared to $1-6 \mathrm{vol} \%$ in air for gasoline ${ }^{22}$ ) and detonability limit range (18.3 - 59 vol\% compared to $1.1-1.3$ vol $\%$ for gasoline ${ }^{23}$ ) make hydrogen also highly interesting as a fuel, which provides hydrogen with another advantage over fossil resources. Furthermore, hydrogen has a very high energy density per weight (J.kg-1) compared to fossil fuels, so that less hydrogen in mass is required to produce the same amount of energy. ${ }^{23}$

Although the hydrogen energy weight density is high, its energy density per volume $\left(\mathrm{J} . \mathrm{L}^{-1}\right)$ is rather low compared to that of gasoline or diesel, which comes from the fact that fossil fuels are liquids and hydrogen a gas under atmospheric conditions. ${ }^{23-25}$ This gaseous phase state gives rise to the technological challenge on how to store hydrogen. A possible solution would be liquefaction, but condensing hydrogen requires temperatures as low as $20 \mathrm{~K}$ or pressures up to $700 \mathrm{bar},{ }^{26}$ which is incredibly impractical on a large scale. Furthermore, storage tanks and pipelines through which the gas is transported are prone to leaking at such high pressures. ${ }^{27}$ Pipeline leakage could not only lead to a loss of hydrogen, but also imposes a safety hazard as a result of the high pressure and the flammability of hydrogen.

Storage and transport techniques under development are based on the reversible binding of hydrogen to a solid chemical carrier. ${ }^{26}$ The binding reversibility, hydrogen capacity, carrier lifetime and robustness to oxidation and temperature and, not in the last place, cost reduction, are still challenges that need solving before this approach can become commercially available. ${ }^{24,} 26$ Overall, more research into the storage and transport of hydrogen is necessary before hydrogen as a fuel is commercially viable. ${ }^{23}$

Nevertheless, the hydrogen market is already sufficiently large with definite economical potential. The global hydrogen market is expected to increase from 43 Mtons in 2010 to 50 Mtons in 2025. ${ }^{28}$ Industry, mainly petrochemical refineries and chemical production such ammonia and methanol, is the largest hydrogen consumer, with 7 Mtons in the EU alone. ${ }^{28}$ According to the 2015 market analysis of the EU, the mobility sector will be the most important driving force for investment in renewable hydrogen, as the automobile industry, passenger cars in particular, is responsible for 
$32 \%$ of the European greenhouse gas emission. Concretely, the automobile industry comprises a hydrogen market potential of 3 to 4.8 Mtons. $y^{-1}$, depending on the scenario considered to predict fuel cell market penetration. ${ }^{28}$

Transformation of this significant fossil fuel market to a sustainable, hydrogen-based one, requires a tremendous amount of effort. Arguably, political and societal-economical motivators are the most important to stimulate this energy transition. The end-user must be able to see the economic and environmental benefits of using hydrogen, otherwise, a hydrogen economy remains unachievable.

\subsection{Hydrogen production from biomass}

\subsubsection{Hydrogen production methods and feedstocks}

An important aspect of the fossil fuel-to-hydrogen transition, is the hydrogen production method and the feedstock. A wide range of hydrogen production methods is already available and used for commercial purposes. These methods can be roughly divided into three categories, depending on the external energy source, i.e., thermal, electrolytic, or photolytic processes ${ }^{29-30}$ (Scheme 1.1). Hydrogen production can also include a biological component, such as bacteria or algae that produce hydrogen.

Thermal or thermochemical processes use heat in the presence or absence of a catalyst to break down feedstock, ${ }^{29-30}$ such as gasification, pyrolysis and steam reforming. ${ }^{31-32}$ Currently, $96 \%$ of the hydrogen originates from natural gas, oil and $\mathrm{coal}^{32}$ and is produced using a thermal process. ${ }^{33}$ Characteristic for these methods are the elevated temperatures ( $473 \mathrm{~K}$ and above) and in some cases high pressures (up to 200 bar). ${ }^{34-35}$ Thermal processing often suffers from high operational and maintenance costs, catalyst deactivation and energy losses. ${ }^{30}$ At the same time, steam reforming is a well-established process, which currently has the highest efficiency compared to the other methods. ${ }^{29-30}$ Although steam reforming has the highest emission, ${ }^{29-30,36}$ it seems the most suitable $\mathrm{H}_{2}$-production method on the short-term.

A long-term option is to produce hydrogen using solar light, ${ }^{29,}{ }^{37-38}$ for instance using photocatalytic water splitting ${ }^{32}$ or photofermentation in the presence of bacteria. ${ }^{30}$ While greenhouse gas emissions with these methods are very low, unfortunately so is the hydrogen 
efficiency. ${ }^{29,32}$ Photofermentation depends on organisms and the hydrogen production rate is typically low. ${ }^{30}$ However, the reactor design is very simple, which therefore reduces material costs. $^{30,32}$ In contrast, photovoltaic water-splitting presents higher hydrogen yields, but it also requires more expensive reactors. ${ }^{36}$

Next to thermal and photolytic processes, hydrogen can be produced using electrolytic methods, of which electrolysis of water is probably the most well-known example. During water electrolysis, water molecules are split into hydrogen and oxygen by means of electricity. Water can be seen as the best possible hydrogen feedstock, as it is widely available ${ }^{39}$ and therefore comes at a low cost price. ${ }^{37}$ Electrolysis, however, is probably one of the more expensive hydrogen production methods, ${ }^{38}$ as it requires highly specialized and complex devices, with a membrane to separate the gas products. ${ }^{32,35}$ At the same time, it is also one of the most promising technologies for renewable hydrogen production, as no by-products are formed in the reaction. ${ }^{29}$ However, the production efficiency is still low compared to thermochemical processes, since large electricity inputs are needed. ${ }^{30}$ Currently, electrolysis is only applied on a small scale,${ }^{24}$ so even though hydrogen produced from water is highly promising, this process is not viable yet for large scale production. An exception is the chlor-alkali process, in which $\mathrm{H}_{2}, \mathrm{Cl}$, and $\mathrm{NaOH}$ are produced from $\mathrm{NaCl}$ and $\mathrm{H}_{2} \mathrm{O}$ using electricity. ${ }^{40}$

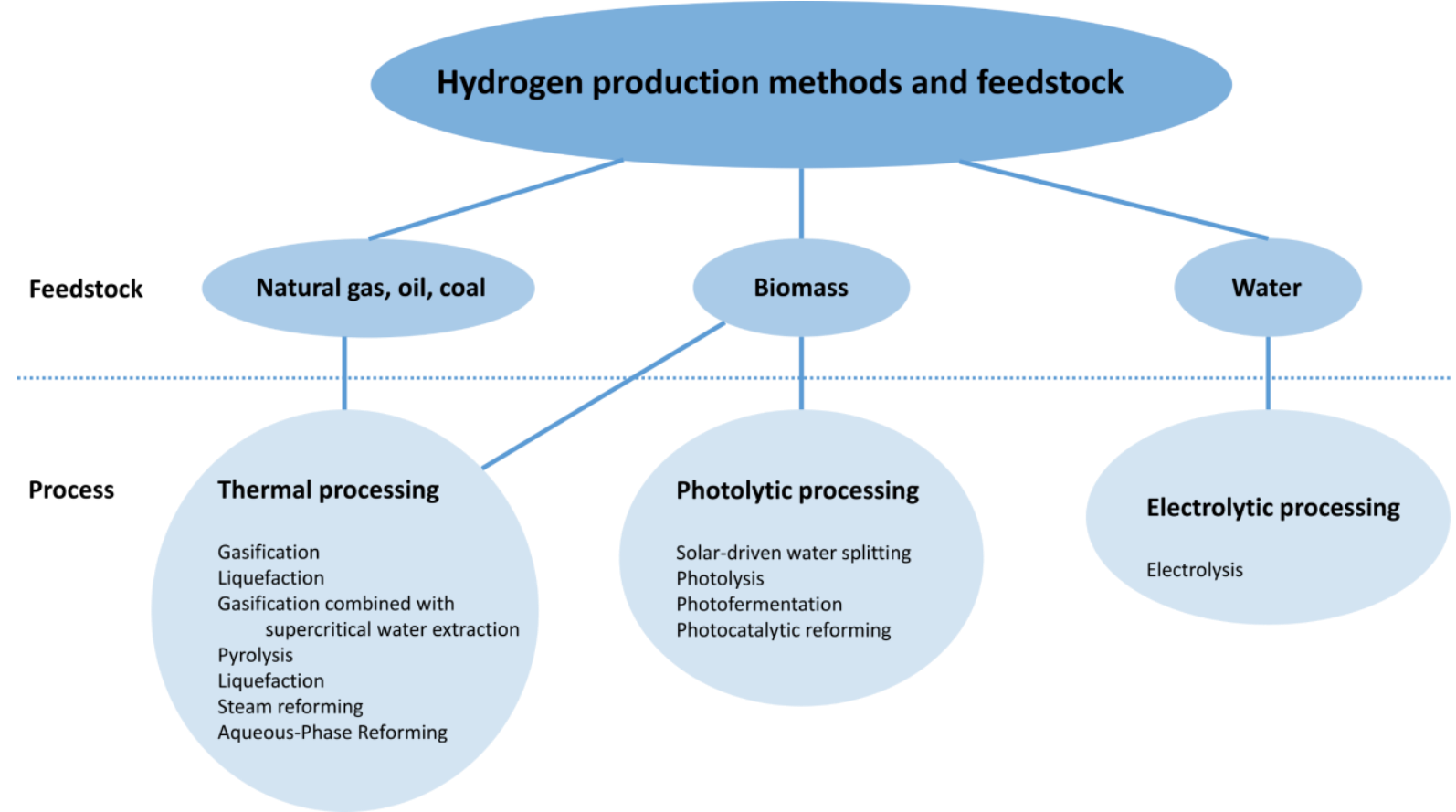

Scheme 1.1 Concise overview of hydrogen production methods and feedstock. 


\subsubsection{Biomass as hydrogen feedstock}

On the shorter term, biomass appears as an alternative and sustainable feedstock for hydrogen production. ${ }^{41}$ Biomass can originate from a wide range of organic materials, such as crops specifically grown for energy production, as well as industrial, forestry, or even municipal waste streams. $^{35,37,42}$ Valorization of these otherwise discarded waste streams provides an interesting opportunity: materials that otherwise would be burnt are now recycled to yield useful hydrogen. ${ }^{41}$

Growing biomass specifically for hydrogen production would aid carbon capture from the atmosphere. ${ }^{41}$ Unlike most organisms, plants containing chlorophyll convert $\mathrm{CO}_{2}$ and water into sugars. When more biomass is grown than used to produce hydrogen, more $\mathrm{CO}_{2}$ is captured from the atmosphere and carbon levels should not continue to increase further. ${ }^{43}$ According to the literature, hydrogen production from biomass could improve the $\mathrm{CO}_{2}$ balance ${ }^{44}$ by up to $30 \% .{ }^{45}$ In addition, biomass feedstock also has a favorable socio-economical effect, as uncultivated lands could be used to produce biomass, boosting the local economy, as well as increasing soil fertility and water retention. 37,45

Using biomass as feedstock for energy generation has also received criticism. Probably the major concerns focus on the competition with growing biomass for food, ${ }^{36}$ as a result of the limited land availability. ${ }^{46}$ This assumption, however, is based on the idea that the biomass has to be grown specifically and solely for hydrogen production, which is not necessarily the case: inedible parts of the crop or, as mentioned earlier, bio-waste streams also represent potential hydrogen feedstock. ${ }^{37}$ When biomass is dedicatedly grown as fuel feedstock, the crop itself has to be selected wisely, as the amount of useful material that can be converted into hydrogen differs from plant to plant. ${ }^{45,47}$ In addition, in a number of countries, crops are burnt for cooking or heating purposes, which is not the most energy efficient use of the biomass. ${ }^{43}$ When the same piece of land would be used to grow crops for hydrogen production, the energy yield per square meter could be increased, leaving thus more acres for food. ${ }^{43}$ Altogether, when well thought-out, biomass as a fuel does not per definition competes with food chains. 


\subsubsection{Biomass reforming processes}

Hydrogen production from biomass always requires an external energy source, ${ }^{36}$ independently of the feedstock and process, which has to be renewable as well for the resulting hydrogen to be considered as a green energy carrier. As other reviews $35,41-42,44-45,48$ have already extensively discussed the different biomass-to-hydrogen production methods, only a brief and broad overview is provided here.

Hydrogen is often produced from biomass using a thermochemical approach. When (fast) pyrolysis is selected, the biomass is converted at a high temperature range of 700 to $950 \mathrm{~K}$ and at a pressure of 1 to 5 bar, forming not only hydrogen, but also $\mathrm{CO}, \mathrm{CH}_{4}$, char and liquid fractions. ${ }^{41}$, 49 To increase the percentage of gas products as well as the hydrogen yield, the reaction temperature can be increased even further to up to $1250 \mathrm{~K}$. Reforming at this temperature is called gasification, ${ }^{49}$ which takes place either in an oxygen or steam atmosphere, where the biomass is partially oxidized, and in some cases reformed. A combination of hydrogen, carbon oxides, methane, carbohydrates and char is produced in this method. ${ }^{41}$

The selectivity towards gas formation, while reducing char and tar, can be promoted using a catalyst. ${ }^{50}$ This procedure is only possible when the water content in the biomass is $35 \%$ or lower. $^{42}$ To process wet biomass streams, gasification can be combined with supercritical water extraction in the absence of oxygen. ${ }^{49}$ Another advantage of this latter method comes from the use of lower reaction temperatures $(647 \mathrm{~K})$, combined with a pressure of $221 \mathrm{bar},{ }^{41,}{ }^{49}$ so that hydrogen is already pressurized and can be stored directly. ${ }^{49}$ Thermodynamic calculations of the equilibrium composition predict that gas products should form at these reaction conditions. ${ }^{41}$

By far more environmentally friendly is hydrogen production using light, which is performed at ambient conditions. ${ }^{41}$ Several bio-organisms, such as cyanobacteria or algae, express an enzyme capable of breaking down the biomass, while releasing hydrogen in the process, for example by biophotolysis and photofermentation. ${ }^{49}$ In biophotolysis, microalgae convert water under the influence of light into hydrogen and oxygen. ${ }^{42}$ Some algae and bacteria can also conduct photosynthesis, in which water and $\mathrm{CO}_{2}$ are reacted into carbohydrates, oxygen and hydrogen. ${ }^{46}$ Photofermentation, however, is performed by bacteria that use organic acids in combination with biomass to produce hydrogen. ${ }^{42}$ 
Still at its infancy, while showing high potential, is photocatalytic biomass reforming. In this process, a semi-conductor catalyst, for example $\mathrm{Pt} / \mathrm{TiO}_{2}$, reduces $\mathrm{H}^{+}$to $\mathrm{H}_{2}$ while (partially) oxidizing an oxygenated carbohydrate, which is a biomass derivate. ${ }^{51-52}$ Photocatalysis is favored compared to conventional water splitting in terms of the Gibbs free energy of reaction. ${ }^{51}$ Recently, hydrogen has been produced from crude glycerol ${ }^{53}$ and ethylene glycol ${ }^{54}$ using this novel method. To cope with the intermittence of solar light, it is also possible to perform the same reaction using electricity, which is collected using wind or another renewable energy source.

Although photo (-chemical) hydrogen production methods are environmentally friendly, they do suffer from low hydrogen production rates and yields. ${ }^{32,} 46$ Therefore thermochemical routes, which are the most established processes, are currently more viable for renewable hydrogen production, provided that the sustainable external energy source is optimally used. Currently, hydrogen production through biomass gasification is found to be the most promising method, due to its high hydrogen yield. ${ }^{30,49,55}$

\subsection{Aqueous-Phase Reforming (APR)}

A relatively new method to potentially produce hydrogen from biomass is Aqueous-Phase Reforming (APR) ${ }^{56-57}$ In this process, which was introduced in 2002, oxygenated carbohydrates with a carbon to oxygen ratio of 1:1, which are biomass derivatives, are converted into $\mathrm{H}_{2}$ and $\mathrm{CO}_{2}$ in water in the liquid state using a heterogeneous catalyst. ${ }^{56}$ Typically, this process occurs at temperatures up to $550 \mathrm{~K}$ and a pressure up to 55 bar to maintain the reaction mixture in the liquid phase. ${ }^{56-57}$ Although APR consists of many reactions, it can be simplified in two main steps:

\section{Cracking}

$$
\mathrm{C}_{\mathrm{n}} \mathrm{H}_{2 \mathrm{y}} \mathrm{O}_{\mathrm{n}} \underset{\mathrm{P}, \mathrm{T}}{\stackrel{\text { catalyst }}{\longrightarrow}} \mathrm{nCO}+\mathrm{yH}_{2}
$$

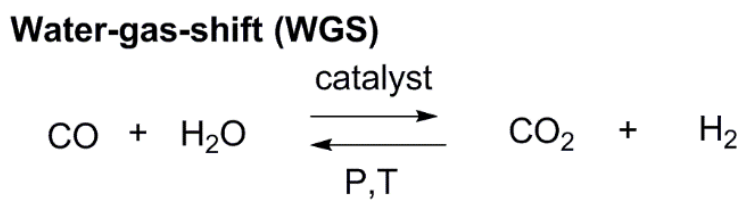

Scheme 1.2 APR can be summarized in two main reactions. First, the substrate is cracked into CO and hydrogen, followed by the water-gas-shift which produces additional hydrogen. 
cracking of the substrate into $\mathrm{CO}$ and $\mathrm{H}_{2}$, followed by the water-gas-shift reaction, where $\mathrm{CO}$ reacts with water to form $\mathrm{CO}_{2}$ and additional $\mathrm{H}_{2}$ (Scheme 1.2). Compared to other thermochemical reforming methods, APR requires a significantly lower temperature, which translates into a lower energy demand. Furthermore, as APR per definition takes place in the liquid phase, the feedstock is much easier to handle, ${ }^{58}$ so that drying the feedstock is no longer required and coking risks are reduced. ${ }^{57,59-61}$

Since APR was first reported by Cortright, Davda and Dumesic, ${ }^{56}$ many scientific reports have been published. They mostly focus on APR catalyst development, elucidation of the complex reaction mechanism, kinetics and to lesser extent on reaction thermodynamics. Usually simple biomass model substrates are considered in these studies, such as ethylene glycol, ${ }^{1-2,62}$ glycerol, ${ }^{62-}$ ${ }^{64}$ and sorbitol. ${ }^{62,65}$<smiles>OCCO</smiles>

Ethylene glycol<smiles>OCC(O)CO</smiles>

Glycerol<smiles>OC[C@@H](O)[C@@H](O)[C@H](O)[C@H](O)CO</smiles>

Sorbitol

Figure 1.1 Biomass model substrates used in APR studies.

Catalytic studies have revealed that noble metals, in particular Pt on oxide supports such as $\mathrm{Al}_{2} \mathrm{O}_{3}$, gave the highest hydrogen yield as well as the highest hydrogen selectivity (up to $100 \%$ ). ${ }^{1-2,57}$ Luo et al. ${ }^{66}$ and Seretis and co-workers ${ }^{63}$ studied the effect of metal loading, temperature and pressure, feedstock concentration and time-on-stream on the reforming of glycerol on Pt-catalysts. Callison et al. found that larger Pt-nanoparticles of $3.5 \mathrm{~nm}$ improved the glycerol conversion to 34\%, and can even influence the product stoichiometry. ${ }^{64}$ As an alternative for catalysts based on noble metals, hybrid catalysts just as Ni-B and Raney Ni-catalysts have been investigated. ${ }^{62}$

The exact APR reaction mechanism (Scheme 1.3) is still under investigation. Huber and Dumesic identified scission of $\mathrm{C}-\mathrm{C}, \mathrm{O}-\mathrm{H}$ and $\mathrm{C}-\mathrm{H}$ bonds as the major steps of the process. ${ }^{67}$ Shabaker et al. ${ }^{2}$ proposed dehydrogenation of ethylene glycol, followed by C-C cleavage to yield gas phase products. Liquid phase products are supposedly formed via either dehydration or isomerization. Apart from coking, both methanation and Fisher-Tropsch synthesis are competing pathways with APR. ${ }^{57,61,67}$ While investigating the influence of the molecular structure of the substrate, Kirilin 


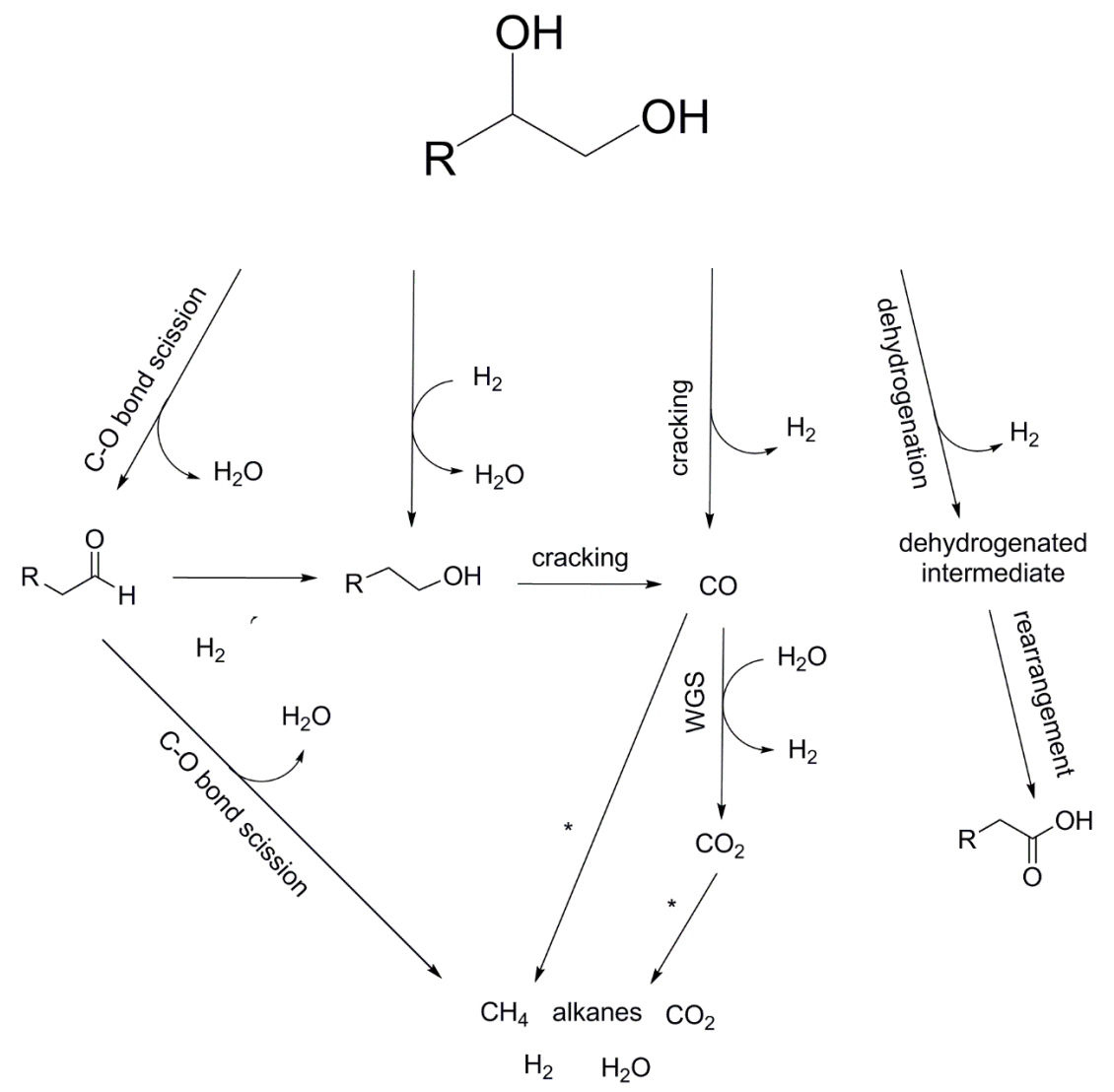

Scheme 1.3 Proposed APR reforming pathways for oxygenated carbohydrates. ${ }^{1-2}$ The arrows indicated with * correspond to methanation and Fisher-Tropsch synthesis, which are unwanted side reactions for hydrogen production using APR.

et al. ${ }^{65}$ showed that APR proceeds from a terminal hydroxyl group. Chiral substrates, such as galacitol and sorbitol, are reformed via comparable pathways with similar hydrogen yields. ${ }^{68}$

To determine the APR reaction conditions at which the highest hydrogen yield and selectivity can be achieved, thermodynamic studies were performed by Seretis et al. ${ }^{69}$ and Davda, ${ }^{1}$ amongst others, where the substrate/water ratio in the inlet stream was taken into account.

Engineering the reactors and process flow has received little attention so far, even though these aspects are vital to eventually design an efficient plant. Formation of gaseous products in a liquid phase, as occurs during APR, gives rise to many challenges, such as local pressure increases, flow instabilities, blocking of the catalyst surface, and unwanted temperature gradients in the reactor. In a properly designed reactor not the catalyst is optimally used, but gas/liquid separation is also performed in-situ. In-situ removal of hydrogen is particularly interesting for APR, as it pulls the 
thermodynamic equilibrium towards the formation of the desired product. Furthermore, smart reactor design allows efficient heat transport while minimizing energy losses. Therefore, the APR reactor system has to be developed in parallel with catalyst optimization: otherwise, even with the most active catalyst APR will not reach its full potential.
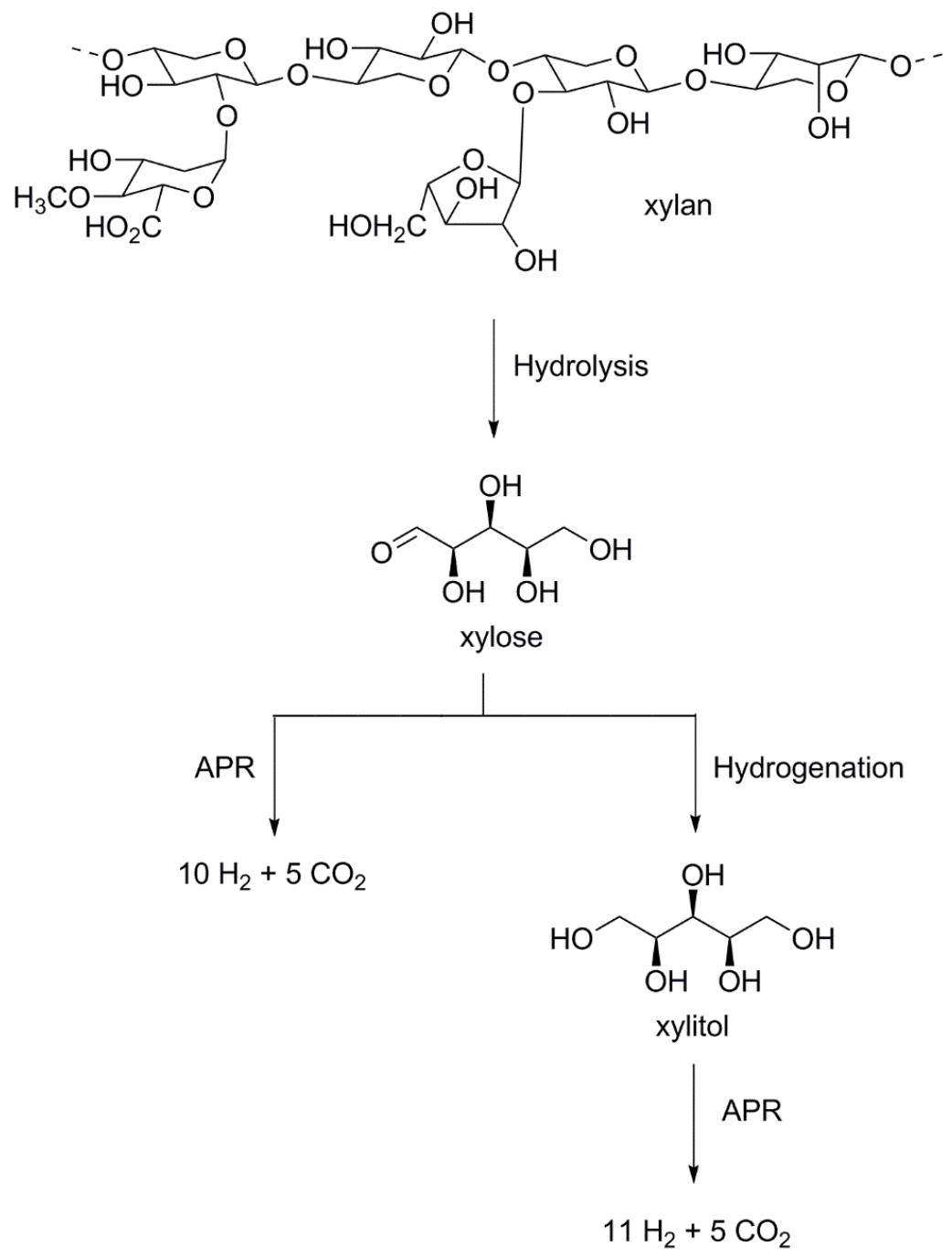

Scheme 1.3 Steps involved in biomass reforming using APR, starting from the hemicellulose (xylan) polymer. The polymer is first hydrolyzed to yield the sugar monomer xylose, followed either by APR or hydrogenation to xylitol. This polyol would then undergo APR in a later stage. Adapted from ${ }^{7}$.

Davda et al. were among the first to present a potential process design, starting from sugars and polyols. ${ }^{57}$ In the first reactor, sugar compounds are hydrogenated to their polyol derivatives, followed by APR in the second reactor. In the third reactor, the levels of CO can be reduced when 
needed. Lastly, the gas separation was performed in a fourth reactor to yield pure hydrogen. A simplified process design was considered in the techno-economical evaluation of APR by Sladkovskiy et al. in $2018,{ }^{70}$ with three reactors. In the first reactor APR is performed, and in the second one the gas and liquid fractions are separated. In the third reactor the gas stream undergoes the WGS. With xylitol as a model feedstock, Murzin and co-workers proposed a process flow including recycling of reactor streams. ${ }^{71}$ In the first reactor, new feedstock, unreacted components and water are mixed and fed to a reactor containing pressurized steam to heat the mixture. In the third reactor, APR takes place. Part of the outlet stream is separated into a gas and a liquid fraction and subsequently the gas fraction was purified by pressure-swing-adsorption (PSA).

On the reactor level, Coronado et al. list several possible configurations. ${ }^{59}$ Batch-scale packed tubular reactors or autoclaves are convenient for lab scale experiments, but these are unsuitable for commercial production. Currently, a continuous packed-bed reactor is now often selected as reactor type to perform APR. For research purposes, however, microreactors provide and excellent platform to investigate several aspects of APR, including thermodynamic properties and the multiphase behavior.

\subsection{Microfluidics as a tool to study and optimize chemical reactions}

The above illustrated that only little research has been conducted from a process technological point of view. Furthermore, the physical aspects of APR, such as transport phenomena, have not yet been investigated in detail. Microreactors would be ideal platform to study these facets of APR. In microfluidic systems that have evolved from microelectronic technology and microelectromechanical systems (MEMS) in the 1980's, ${ }^{72-73}$ small volumes of fluids are manipulated at the micro- or even nanoscale. ${ }^{74-75}$ Typically, fluid channels with micrometer dimensions $^{76}$ are fabricated in silicon or glass substrates using etching processes in a cleanroom environment. ${ }^{72}$ System miniaturization offers several practical advantages: the systems are portable and can integrate several functionalities in a single microdevice. ${ }^{74}$ Furthermore, only small sample volumes are required ${ }^{74}$ so that safety is increased ${ }^{75}$ and operational costs are reduced while working with hazardous and/or expensive chemicals. Furthermore, transport phenomena benefit from the large surface-to-volume ratio of microreactors. ${ }^{75}$ 


\subsubsection{Navier-Stokes equation and the laminar flow regime}

Handling fluids at such a small scale also affects the fluid dynamics, since at the microscale viscous forces typically dominate over gravitational and inertial forces. The fluid flow can be described by the Navier-Stokes equation, assuming an incompressible fluid and the continuity equation.

$$
\begin{aligned}
& \rho(\vec{u} \cdot \nabla) \vec{u}=-\nabla P+\nabla \cdot \mu\left[(\nabla \vec{u})+(\nabla \vec{u})^{T}\right] \\
& \nabla \cdot(\rho \vec{u})=0
\end{aligned}
$$

where $\rho$ is the density of the fluid, $\vec{u}$ the average mean velocity, $P$ the pressure, and $\mu$ the viscosity of the fluid. From Equation 1, the Reynolds number $(R e)$, which is the ratio between the inertial and viscous forces can be derived. In microfluidic systems, $R e$ is typically lower than $2000,{ }^{76}$ corresponding to a laminar flow regime, whereas macrosystems, or systems with fluids of low viscosity often exhibit turbulent flow.

\subsubsection{Heat and mass transport at the microscale}

While the presence of a laminar flow provides better control on the fluid flows, mixing is compromised at the microscale. In general, mixing occurs through two main mechanisms: convection and diffusion. In microfluidics, convection between the fluidic "layers" is non-existing, and therefore diffusion, which scales with $L^{2}$, (with $L$ the characteristic length of the device) is the only driving force for mixing. ${ }^{75}$ Still, mass transport is enhanced compared to large scale systems, as the typical diffusion length scale is several orders of magnitude smaller in microsystems. ${ }^{73}$

Besides mass transport, heat transfer is also essential for chemical processes: heat is either produced or used for reaction. Overall, thermal energy has to be transferred to the fluid relatively fast, to prevent thermal runaway or supply energy to heat-limited reaction rates. In microfluidics, heat transfer is more efficient than in large scale systems as a result of the large surface-to-volumeratio. ${ }^{75}$ In other words, the surface through which heat has to be withdrawn or applied is sufficiently large compared to the reactor volume, so that the time scale of heat transport is usually shorter than the heat production/consumption rate. 


\subsection{Multiphase catalytic microreactors}

It is for these fluid and transport characteristics that microreactors are particularly interesting to study APR: external mass transfer limitations that exist in continuous packed-bed reactors are circumvented and the efficient heat transfer should accommodate the endothermic APR reaction. Another advantage of miniaturization is the control of the gas/liquid/solid multiphase system, which is formed during APR.

The catalyst can be incorporated in a similar fashion as for large scale reactors: solid catalysts can be introduced as a packed bed, ${ }^{77-78}$ a monolith (foam), ${ }^{79-80}$ or as a thin layer on the microchannel wall. ${ }^{81-82}$ For microfluidic applications, especially for multiphase systems, a thin-layer configuration is the preferred option. In this case, the reactor can be operated at relatively high flow rates, without a significant increase in the pressure drop, and the catalyst can easily be separated from the reactants, products, and solvents. ${ }^{83}$ Such a thin-film microreactor for APR has been developed by D'Angelo et al. ${ }^{82}$, who reported a hydrogen selectivity three times higher than for a fixed bed reactor.

In APR, next to the solid catalyst and the liquid reaction mixture, a third gaseous phase is formed by APR products (including $\mathrm{H}_{2}, \mathrm{CO}, \mathrm{CO}_{2}$ and $\mathrm{CH}_{4}$ ). The resulting gas/liquid flow can be characterized by, for example, the chordal void fraction $\alpha_{\text {chordal }}$ :

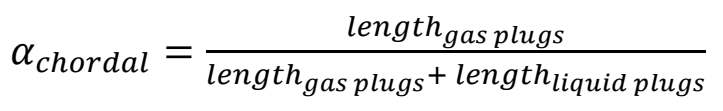

which is the ratio between the gas plug length and the total fluid length. Depending on the chordal void fraction, several gas/liquid flow regimes can be distinguished, such as bubbly flow, Taylor flow, annular flow and a dry flow for increasing void fractions. ${ }^{84}$

\subsubsection{Velocity profile and boundary conditions}

The presence of a gas phase besides a liquid and a solid phase can affect the fluid dynamics in the microreactor. Usually the fluid flow in a microreactor is pressure driven, resulting in a parabolic velocity profile, also known as Hagen-Poiseuille flow with the maximum velocity at the center of the channel. 


$$
u_{z}=-\frac{1}{4 \mu} \frac{\partial p}{\partial z}\left(R^{2}-r^{2}\right)
$$

where $u_{z}$ is the velocity at position $z$ in the microchannel, $R$ the radius of the microchannel and $r$ the radial position in the channel at which the velocity is calculated. This equation is valid assuming a no-slip boundary condition, i.e., no velocity at the microchannel wall. A no-slip boundary condition exists for a wetted-wall, for example water flowing through a silicon microchannel. The shear force at the channel wall is relatively large, due to the drag of the wall on the liquid.

In a gas/liquid system, in particular when bubbles are trapped in or pinned to small surface defects, the fluid dynamics are different. As gas is hydrophobic, and water hydrophilic, the liquid has a different contact angle on the gaseous surfaces than on the bare oxidized silicon wall. When the contact angle becomes equal to or larger than $150^{\circ}$, the surface is considered "superhydrophobic". A well-known example of a superhydrophic surface is a lotus leaf, where a water droplet either sits or slides off when it contacts the leaf surface.

The viscous friction of the liquid on these superhydrophic surfaces is reduced compared to wetted surfaces, and so is the drag at the channel wall. ${ }^{6,85-86}$ Instead of a no-slip velocity boundary

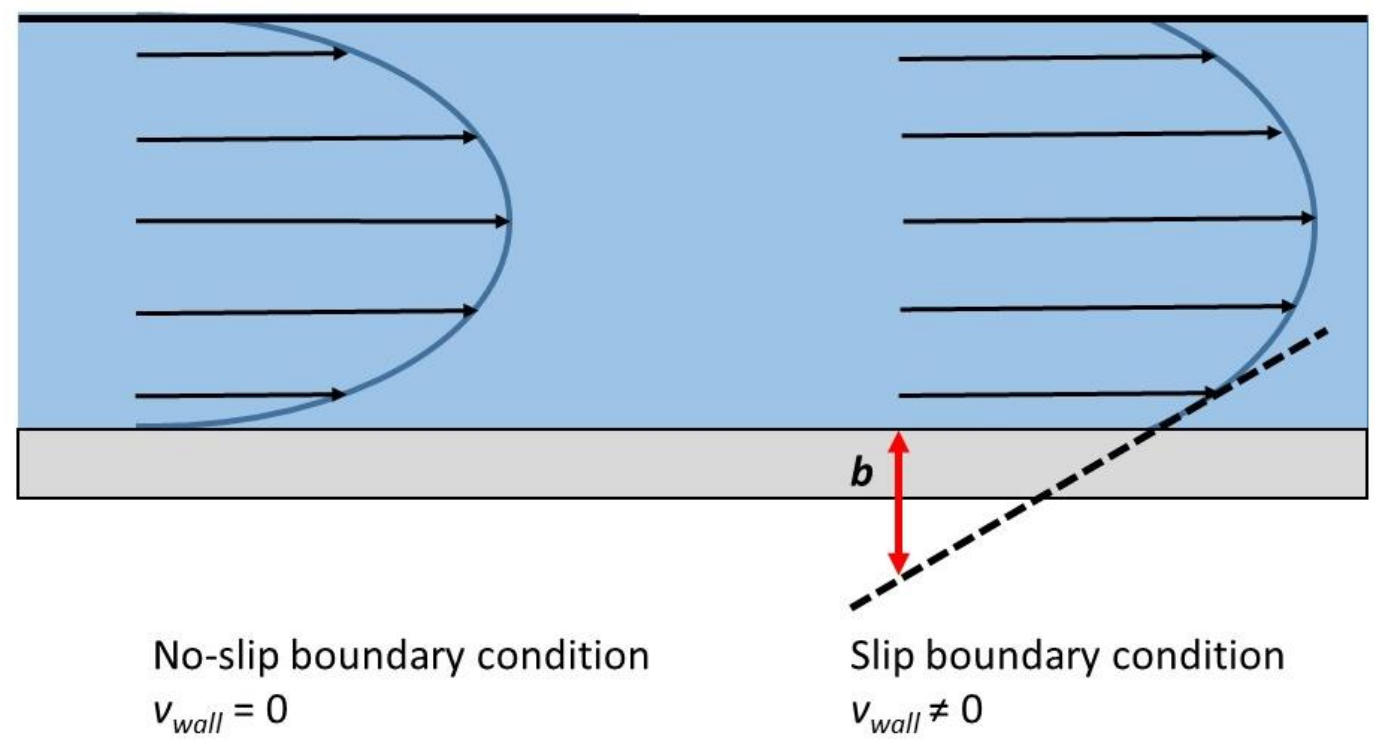

Figure 6.2 A no-slip and a slip boundary condition in a completely wetted hydrophilic microchannel with a laminar flow and a parabolic velocity profile. ${ }^{6}$ 
condition, a slip condition now exists at the superhydrophobic gas/liquid interface, with a net velocity at the wall, ${ }^{85}$ and a reduced shear force..$^{6,87}$

The slip is quantified by the slip length $b$ :

$$
b=\delta\left(\frac{\mu_{l}}{\mu_{g}}-1\right) \cong \delta \frac{\mu_{l}}{\mu_{g}}
$$

with $\delta$ is the gas layer thickness, and $\mu$ the viscosity of the liquid (l) and the gas $(\mathrm{g}){ }^{88-89}$ This expression clearly dictates that in order to create a slip length in the order of magnitude as that of the microreactor, the viscosity difference must be sufficiently large, as is often the case for a gas and a liquid. ${ }^{90}$

\subsubsection{Influence of the slip velocity on transport phenomena}

One of the consequences of the slip velocity is a reduction in the boundary layer thickness. As discussed earlier, viscous forces dominate inertial and gravitational forces in a microsystem, for $R e<2000$. The high shear force at the microfluidic wall due to the no-slip boundary condition then results in a zero velocity at the wall. Closer to the center of the channel, however, the velocity has a much higher value, resulting in a sharp velocity gradient close the microchannel wall. ${ }^{91-92}$ The region where this sharp gradient takes place is called the boundary layer. A similar boundary exists for the heat and mass transfer.

In contrast, on a no-shear wall with a slip boundary condition, the velocity at the interface is unequal to 0 . Therefore, the gradient between the velocity at the wall and closer to the center of the fluidic channel is less steep and the thickness of the boundary layer is reduced. Furthermore, the value for the momentum, heat and mass transfer at the channel wall has a closer resemblance to that of the bulk properties than for a no-slip boundary condition. As a result, the interfacial mass transfer is enhanced in a system with a slip velocity greater than 0 .

\subsection{Thesis outline}

This thesis aims to investigate the hydrogen production from oxygenated carbohydrates using Aqueous-Phase Reforming. In particular, the thermodynamic and engineering aspects involved to 
optimally design the APR process are investigated at the microscale. Throughout this work, four biomass model solutions are considered, consisting of ethylene glycol, glycerol, xylose and xylitol. An introduction to the topic is given in this chapter.

In Chapter 2 the APR energy requirement is calculated in terms of the enthalpy and Gibbs free energy of reaction using data from both the literature and ASPEN Plus. To properly determine these properties, the phase state of the different biomass model solutions and products are evaluated at APR reaction conditions.

To validate the phase behavior of the biomass model solutions, a high-pressure and hightemperature microfluidic set-up is presented in Chapter 3. Furthermore, the multiple gas/liquid flow regimes that form after boiling has occurred are characterized as a function of the chordal void fraction. The void fraction at which one flow regime transits to another, can be used as an prediction of the flow regime that can occur as a result of gas formation during APR.

In Chapter 4 the momentum and the heat and mass transport are numerically investigated using a 2D-representation of the microfluidic device described in Chapter 2. In COMSOL Multiphysics 5.3a, APR of a $10 \mathrm{wt} \%$ glycerol/water solutions is studied, assuming the gaseous products form a bubble mattress at the catalytic layer.

This model is extended to a 3D-model in Chapter 5. Furthermore, a second generation microfluidic device is developed in which the location of bubble nucleation can be controlled experimentally with the help of hydrophobic micropits. As bubbles seem to affect the transport phenomena, this microfluidic design is a first step to control the bubble nucleation and thus to optimize the heat and mass transfer.

To perform APR in a microfluidic device, a heterogeneous catalyst must be incorporated in the fluidic channel. In Chapter 6 both washcoating and spark ablation, a wet chemical and a physical deposition technique, are investigated to deposit a $\mathrm{TiO}_{2}$ support and $\mathrm{Cu} / \mathrm{CuO}$ active phase respectively in the microfluidic channel.

When APR is performed, the gas and liquid phases must be separated before hydrogen is purified. In Chapter 7 a modular gas/liquid microseparator based on micropillars is presented for the downstream processing of APR reactor outlet streams. Special attention is given to the minimization of the dead volume in the separation module. 
Although APR is less energy demanding than the conventional hydrogen producing methods, the energy demand, which is supplied to the system as heat, is still significant. A more sustainable production method would be based on, for example, solar energy. In Chapter 8 first steps towards the photocatalytic reforming of biomass are reported. In particular, the required bandgap of the photocatalyst and the reaction mechanism are investigated. A microfluidic device is proposed in which photocatalytic reforming can be studied electrochemically and in which the products of the half-reactions can be analyzed separately.

The multiphase reaction engineering aspects of APR are concluded in Chapter 9, followed by a short perspective on how APR could eventually be implemented for industrial hydrogen production.

\section{References}

1. Davda, R. R.; Shabaker, J. W.; Huber, G. W.; Cortright, R. D.; Dumesic, J. A., Aqueousphase reforming of ethylene glycol on silica-supported metal catalysts. Applied Catalysis B: Environmental 2003, 43 (1), 13-26.

2. Shabaker, J. W.; Dumesic, J. A., Kinetics of Aqueous-Phase Reforming of Oxygenated Hydrocarbons: Pt/A12O3 and Sn-Modified Ni Catalysts. Industrial \& Engineering Chemistry Research 2004, 43 (12), 3105-3112.

3. NASA Global Climate Change. https://climate.nasa.gov/ (accessed 27-7-2018).

4. Allen, M. R.; Dube, O. P.; Solecki, W.; Aragon-Durand, F.; Cramer, W.; Humphreys, S.; Kainuma, M.; Kala, J.; Mahowald, N.; Mulugetta, Y.; Perez, R.; Wairiu, M.; Zickfeld, K. Framing and Context; IPCC: 2018.

5. Laboratory, E. S. R. CO2 at NOAA's Mauna Loa Observatory reaches new milestone: Tops 400 ppm. https://www.esrl.noaa.gov/gmd/news/7074.html.

6. Ou, J.; Perot, B.; Rothstein, J. P., Laminar drag reduction in microchannels using ultrahydrophobic surfaces. Physics of Fluids 2004, 16 (12), 4635-4643.

7. $\quad$ Kirilin, A. V.; Hasse, B.; Tokarev, A. V.; Kustov, L. M.; Baeva, G. N.; Bragina, G. O.; Stakheev, A. Y.; Rautio, A.-R.; Salmi, T.; Etzold, B. J. M.; Mikkola, J.-P.; Murzin, D. Y., Aqueous-phase reforming of xylitol over Pt/C and Pt/TiC-CDC catalysts: catalyst characterization and catalytic performance. Catalysis Science \& Technology 2014, 4 (2), 387-401. 
8. Schwalm, C. R.; Anderegg, W. R. L.; Michalak, A. M.; Fisher, J. B.; Biondi, F.; Koch, G.; Litvak, M.; Ogle, K.; Shaw, J. D.; Wolf, A.; Huntzinger, D. N.; Schaefer, K.; Cook, R.; Wei, Y. X.; Fang, Y. Y.; Hayes, D.; Huang, M. Y.; Jain, A.; Tian, H. Q., Global patterns of drought recovery. Nature 2017, 548 (7666), 202-+.

9. Amrith, S. S., Risk and the South Asian monsoon. Climatic Change 2018, 151 (1), 17-28.

10. Nations, U. Global Issues Overview. http://www.un.org/en/sections/issues-depth/globalissues-overview/.

11. Nations, U., World Population Prospects: The 2017 Revision. Affairs, D. o. E. a. S., Ed. New York, 2017.

12. Sorrell, S., Reducing energy demand: A review of issues, challenges and approaches. Renewable \& Sustainable Energy Reviews 2015, 47, 74-82.

13. Brounen, D.; Kok, N.; Quigley, J. M., Residential energy use and conservation: Economics and demographics. European Economic Review 2012, 56 (5), 931-945.

14. Administration, U. S. E. I., International Energy Outlook 2017. 2017.

15. Administration, U. S. E. I., U.S. energy consumption by energy source. Monthyl Energy Review, 2018.

16. Stephens, E.; Ross, I. L.; Mussgnug, J. H.; Wagner, L. D.; Borowitzka, M. A.; Posten, C.; Kruse, O.; Hankamer, B., Future prospects of microalgal biofuel production systems. Trends in Plant Science 2010, 15 (10), 554-564.

17. Agency, I. E. Glossary. https://www.iea.org/about/glossary/r/ (accessed 12-12-2018).

18. dictionary, C., Collins dictionary. 2018.

19. Orecchini, F., The era of energy vectors. Int. J. Hydrogen Energy 2006, 31 (14), 19511954.

20. Suberu, M. Y.; Mustafa, M. W.; Bashir, N., Energy storage systems for renewable energy power sector integration and mitigation of intermittency. Renewable \& Sustainable Energy Reviews 2014, 35, 499-514.

21. Blanco, H.; Faaij, A., A review at the role of storage in energy systems with a focus on Power to Gas and long-term storage. Renewable \& Sustainable Energy Reviews 2018, 81, 10491086.

22. Armaroli, N.; Balzani, V., The Hydrogen Issue. Chemsuschem 2011, 4 (1), 21-36. 
23. Balat, M., Potential importance of hydrogen as a future solution to environmental and transportation problems. Int. J. Hydrogen Energy 2008, 33 (15), 4013-4029.

24. Sharma, S.; Ghoshal, S. K., Hydrogen the future transportation fuel: From production to applications. Renewable \& Sustainable Energy Reviews 2015, 43, 1151-1158.

25. Martens, J. A.; Bogaerts, A.; De Kimpe, N.; Jacobs, P. A.; Marin, G. B.; Rabaey, K.; Saeys, M.; Verhelst, S., The Chemical Route to a Carbon Dioxide Neutral World. Chemsuschem 2017, 10 (6), 1039-1055.

26. Rusman, N. A. A.; Dahari, M., A review on the current progress of metal hydrides material for solid-state hydrogen storage applications. Int. J. Hydrogen Energy 2016, 41 (28), 12108-12126. 27. Ball, M.; Weeda, M., The hydrogen economy - Vision or reality? Int. J. Hydrogen Energy 2015, 40 (25), 7903-7919.

28. CertifHy Overview of the market segmentation for hydrogen across potential customer groups, based on key application areas; 2015.

29. Dincer, I.; Acar, C., Innovation in hydrogen production. Int. J. Hydrogen Energy 2017, 42 (22), 14843-14864.

30. Veras, T. D.; Mozer, T. S.; dos Santos, D.; Cesar, A. D., Hydrogen: Trends, production and characterization of the main process worldwide. Int. J. Hydrogen Energy 2017, 42 (4), 20182033.

31. Besancon, B. M.; Hasanov, V.; Imbault-Lastapis, R.; Benesch, R.; Barrio, M.; Mølnvik, M. J., Hydrogen quality from decarbonized fossil fuels to fuel cells. Int. J. Hydrogen Energy 2009, 34 (5), 2350-2360.

32. Nikolaidis, P.; Poullikkas, A., A comparative overview of hydrogen production processes. Renewable \& Sustainable Energy Reviews 2017, 67, 597-611.

33. Shahid, M.; Bidin, N.; Mat, Y.; Ullah, M. I., Production and Enhancement of Hydrogen From Water: A Review. Journal of Energy Resources Technology-Transactions of the Asme 2012, $134(3)$.

34. Udengaard, N. R., Hydrogen production by steam reforming of hydrocarbons. Abstracts of Papers of the American Chemical Society 2004, 228, U682-U682.

35. Holladay, J. D.; Hu, J.; King, D. L.; Wang, Y., An overview of hydrogen production technologies. Catal. Today 2009, 139 (4), 244-260. 
36. Bartels, J. R.; Pate, M. B.; Olson, N. K., An economic survey of hydrogen production from conventional and alternative energy sources. Int. J. Hydrogen Energy 2010, 35 (16), 8371-8384.

37. Hosseini, S. E.; Wahid, M. A., Hydrogen production from renewable and sustainable energy resources: Promising green energy carrier for clean development. Renewable and Sustainable Energy Reviews 2016, 57, 850-866.

38. Chaubey, R.; Sahu, S.; James, O. O.; Maity, S., A review on development of industrial processes and emerging techniques for production of hydrogen from renewable and sustainable sources. Renewable and Sustainable Energy Reviews 2013, 23, 443-462.

39. Acar, C.; Dincer, I.; Naterer, G. F., Review of photocatalytic water-splitting methods for sustainable hydrogen production. International Journal of Energy Research 2016, 40 (11), 14491473.

40. Crook, J.; Mousavi, A., The chlor-alkali process: A review of history and pollution. Environmental Forensics 2016, 17 (3), 211-217.

41. Navarro, R. M.; Sánchez-Sánchez, M. C.; Alvarez-Galvan, M. C.; Valle, F. d.; Fierro, J. L. G., Hydrogen production from renewable sources: biomass and photocatalytic opportunities. Energy \& Environmental Science 2009, 2 (1), 35-54.

42. Ni, M.; Leung, D. Y. C.; Leung, M. K. H.; Sumathy, K., An overview of hydrogen production from biomass. Fuel Process. Technol. 2006, 87 (5), 461-472.

43. Fresco, L. O., Biomass for food or fuel. 2006.

44. Tanksale, A.; Beltramini, J. N.; Lu, G. M., A review of catalytic hydrogen production processes from biomass. Renewable \& Sustainable Energy Reviews 2010, 14 (1), 166-182.

45. Balat, H.; Kirtay, E., Hydrogen from biomass - Present scenario and future prospects. Int. J. Hydrogen Energy 2010, 35 (14), 7416-7426.

46. Saxena, R. C.; Adhikari, D. K.; Goyal, H. B., Biomass-based energy fuel through biochemical routes: A review. Renewable and Sustainable Energy Reviews 2009, 13 (1), 167-178. 47. Vassilev, S. V.; Baxter, D.; Andersen, L. K.; Vassileva, C. G., An overview of the chemical composition of biomass. Fuel 2010, 89 (5), 913-933.

48. Levin, D. B.; Chahine, R., Challenges for renewable hydrogen production from biomass. Int. J. Hydrogen Energy 2010, 35 (10), 4962-4969.

49. Kirtay, E., Recent advances in production of hydrogen from biomass. Energy Convers. Manage. 2011, 52 (4), 1778-1789. 
50. Elliott, D. C., Catalytic hydrothermal gasification of biomass. Biofuels Bioproducts \& Biorefining-Biofpr 2008, 2 (3), 254-265.

51. Melo, M. D.; Silva, L. A., Photocatalytic Production of Hydrogen: an Innovative Use for Biomass Derivatives. Journal of the Brazilian Chemical Society 2011, 22 (8), 1399-1406.

52. Lianos, P., Review of recent trends in photoelectrocatalytic conversion of solar energy to electricity and hydrogen. Applied Catalysis B-Environmental 2017, 210, 235-254.

53. Reddy, N. L.; Cheralathan, K. K.; Kumari, V. D.; Neppolian, B.; Venkatakrishnan, S. M., Photocatalytic Reforming of Biomass Derived Crude Glycerol in Water: A Sustainable Approach for Improved Hydrogen Generation Using $\mathrm{Ni}(\mathrm{OH})(2)$ Decorated $\mathrm{TiO} 2$ Nanotubes under Solar Light Irradiation. Acs Sustainable Chemistry \& Engineering 2018, 6 (3), 3754-3764.

54. Li, F. Y.; Gu, Q.; Niu, Y.; Wang, R. Z.; Tong, Y. C.; Zhu, S. Y.; Zhang, H. L.; Zhang, Z. Z.; Wang, X. X., Hydrogen evolution from aqueous-phase photocatalytic reforming of ethylene glycol over Pt/TiO2 catalysts: Role of Pt and product distribution. Appl. Surf. Sci. 2017, 391, 251258.

55. Balat, M.; Balat, M., Political, economic and environmental impacts of biomass-based hydrogen. Int. J. Hydrogen Energy 2009, 34 (9), 3589-3603.

56. Cortright, R. D.; Davda, R. R.; Dumesic, J. A., Hydrogen from catalytic reforming of biomass-derived hydrocarbons in liquid water. Nature 2002, 418 (6901), 964-967.

57. Davda, R. R.; Shabaker, J. W.; Huber, G. W.; Cortright, R. D.; Dumesic, J. A., A review of catalytic issues and process conditions for renewable hydrogen and alkanes by aqueous-phase reforming of oxygenated hydrocarbons over supported metal catalysts. Applied Catalysis BEnvironmental 2005, 56 (1-2), 171-186.

58. Lin, Y. C., Catalytic valorization of glycerol to hydrogen and syngas. Int. J. Hydrogen Energy 2013, 38 (6), 2678-2700.

59. Coronado, I.; Stekrova, M.; Reinikainen, M.; Simell, P.; Lefferts, L.; Lehtonen, J., A review of catalytic aqueous-phase reforming of oxygenated hydrocarbons derived from biorefinery water fractions. Int. J. Hydrogen Energy 2016, 41 (26), 11003-11032.

60. Mohanty, P.; Pant, K. K.; Mittal, R., Hydrogen generation from biomass materials: challenges and opportunities. Wiley Interdisciplinary Reviews-Energy and Environment 2015, 4 (2), 139-155. 
61. Chheda, J. N.; Huber, G. W.; Dumesic, J. A., Liquid-phase catalytic processing of biomassderived oxygenated hydrocarbons to fuels and chemicals. Angewandte Chemie-International Edition 2007, 46 (38), 7164-7183.

62. Guo, Y.; Liu, X. H.; Azmat, M. U.; Xu, W. J.; Ren, J. W.; Wang, Y. Q.; Lu, G. Z., Hydrogen production by aqueous-phase reforming of glycerol over Ni-B catalysts. Int. J. Hydrogen Energy 2012, 37 (1), 227-234.

63. Seretis, A.; Tsiakaras, P., Aqueous phase reforming (APR) of glycerol over platinum supported on A12O3 catalyst. Renewable Energy 2016, 85, 1116-1126.

64. Callison, J.; Subramanian, N. D.; Rogers, S. M.; Chutia, A.; Gianolio, D.; Catlow, C. R. A.; Wells, P. P.; Dimitratos, N., Directed aqueous-phase reforming of glycerol through tailored platinum nanoparticles. Applied Catalysis B-Environmental 2018, 238, 618-628.

65. Kirilin, A. V.; Tokarev, A. V.; Kustov, L. M.; Salmi, T.; Mikkola, J. P.; Murzin, D. Y., Aqueous phase reforming of xylitol and sorbitol: Comparison and influence of substrate structure. Applied Catalysis a-General 2012, 435, 172-180.

66. Luo, N. J.; Fu, X. W.; Cao, F. H.; Xiao, T. C.; Edwards, P. P., Glycerol aqueous phase reforming for hydrogen generation over Pt catalyst - Effect of catalyst composition and reaction conditions. Fuel 2008, 87 (17-18), 3483-3489.

67. Huber, G. W.; Dumesic, J. A., An overview of aqueous-phase catalytic processes for production of hydrogen and alkanes in a biorefinery. Catal. Today 2006, 111 (1), 119-132.

68. Godina, L. I.; Kirilin, A. V.; Tokarev, A. V.; Murzin, D. Y., Aqueous Phase Reforming of Industrially Relevant Sugar Alcohols with Different Chiralities. Acs Catalysis 2015, 5 (5), 29893005 .

69. Seretis, A.; Tsiakaras, P., A thermodynamic analysis of hydrogen production via aqueous phase reforming of glycerol. Fuel Process. Technol. 2015, 134, 107-115.

70. Sladkovskiy, D. A.; Godina, L. I.; Semikin, K. V.; Sladkovskaya, E. V.; Smirnova, D. A.; Murzin, D. Y., Process design and techno-economical analysis of hydrogen production by aqueous phase reforming of sorbitol. Chemical Engineering Research \& Design 2018, 134, 104-116.

71. Murzin, D. Y.; Garcia, S.; Russo, V.; Kilpio, T.; Godina, L. I.; Tokarev, A. V.; Kirilin, A. V.; Simakova, I. L.; Poulston, S.; Sladkovskiy, D. A.; Warna, J., Kinetics, Modeling, and Process Design of Hydrogen Production by Aqueous Phase Reforming of Xylitol. Industrial \& Engineering Chemistry Research 2017, 56 (45), 13241-13254. 
72. Temiz, Y.; Lovchik, R. D.; Kaigala, G. V.; Delamarche, E., Lab-on-a-chip devices: How to close and plug the lab? Microelectron. Eng. 2015, 132, 156-175.

73. Vladisavljević, G. T.; Khalid, N.; Neves, M. A.; Kuroiwa, T.; Nakajima, M.; Uemura, K.; Ichikawa, S.; Kobayashi, I., Industrial lab-on-a-chip: Design, applications and scale-up for drug discovery and delivery. Advanced Drug Delivery Reviews 2013, 65 (11), 1626-1663.

74. Zhang, J.; Yan, S.; Yuan, D.; Alici, G.; Nguyen, N. T.; Warkiani, M. E.; Li, W. H., Fundamentals and applications of inertial microfluidics: a review. Lab on a Chip 2016, 16 (1), 1034.

75. Wiles, C.; Watts, P., Continuous flow reactors, a tool for the modern synthetic chemist. Eur. J. Org. Chem. 2008, (10), 1655-1671.

76. Worner, M., Numerical modeling of multiphase flows in microfluidics and micro process engineering: a review of methods and applications. Microfluidics and Nanofluidics 2012, 12 (6), 841-886.

77. Iliuta, I.; Hamidipour, M.; Schweich, D.; Larachi, F., Two-phase flow in packed-bed microreactors: Experiments, model and simulations. Chem. Eng. Sci. 2012, 73, 299-313.

78. He, W.; Fang, Z.; Zhang, K.; Tu, T.; Lv, N.; Qiu, C.; Guo, K., A novel micro-flow system under microwave irradiation for continuous synthesis of 1,4-dihydropyridines in the absence of solvents via Hantzsch reaction. Chem. Eng. J. 2018, 331, 161-168.

79. Zhu, L.; Fu Tan, C.; Gao, M.; Ho, G. W., Design of a Metal Oxide-Organic Framework (MoOF) Foam Microreactor: Solar-Induced Direct Pollutant Degradation and Hydrogen Generation. Adv. Mater. 2015, 27 (47), 7713-7719.

80. Chen, G.; Zhu, X.; Chen, R.; Liao, Q.; Ye, D.; Feng, H.; Liu, J.; Liu, M., Gas-liquid-solid monolithic microreactor with Pd nanocatalyst coated on polydopamine modified nickel foam for nitrobenzene hydrogenation. Chem. Eng. J. 2018, 334, 1897-1904.

81. Tanimu, A.; Jaenicke, S.; Alhooshani, K., Heterogeneous catalysis in continuous flow microreactors: A review of methods and applications. Chem. Eng. J. 2017, 327, 792-821.

82. D'Angelo, M. F. N.; Ordomsky, V.; Paunovic, V.; van der Schaaf, J.; Schouten, J. C.; Nijhuis, T. A., Hydrogen Production through Aqueous-Phase Reforming of Ethylene Glycol in a Washcoated Microchannel. ChemSusChem 2013, 6 (9), 1708-1716.

83. Kapteijn, F.; Nijhuis, T. A.; Heiszwolf, J. J.; Moulijn, J. A., New non-traditional multiphase catalytic reactors based on monolithic structures. Catal. Today 2001, 66 (2), 133-144. 
84. Zhao, C. X.; Middelberg, A. P. J., Two-phase microfluidic flows. Chem. Eng. Sci. 2011, 66 (7), 1394-1411.

85. Bocquet, L.; Lauga, E., A smooth future? Nature Materials 2011, 10, 334.

86. Bocquet, L.; Barrat, J. L., Flow boundary conditions from nano- to micro-scales. Soft Matter 2007, 3 (6), 685-693.

87. Rao, I. J.; Rajagopal, K. R., The effect of the slip boundary condition on the flow of fluids in a channel. Acta Mechanica 1999, 135 (3), 113-126.

88. Vinogradova, O. I.; Dubov, A. L., Superhydrophobic textures for microfluidics. Mendeleev Commun. 2012, 22 (5), 229-236.

89. Hendy, S. C.; Lund, N. J., Effective slip lengths for flows over surfaces with nanobubbles: the effects of finite slip. Journal of Physics-Condensed Matter 2009, 21 (14).

90. Ou, J.; Rothstein, J. P., Direct velocity measurements of the flow past drag-reducing ultrahydrophobic surfaces. Physics of Fluids 2005, 17 (10).

91. Burr, K. P., Akylas, T.R., Mei, C.C CHAPTER TWO TWO-DIMENSIONAL LAMINAR BOUNDARY LAYERS. http://web.mit.edu/fluidsmodules/www/highspeed_flows/ver2/bl_Chap2.pdf.

92. Boundary layers. http://www.cns.gatech.edu/ predrag/GTcourses/PHYS-442104/lautrup/7.6/boundaries.pdf. 


\title{
2 \\ Thermodynamics of APR
}

\begin{abstract}
Hydrogen is a promising renewable energy source that can be produced from biomass using aqueous-phase reforming (APR). Here, using data obtained from AspenPlus and the literature, we evaluated the phase state, temperature-dependent enthalpy, and Gibbs free energy for the APR of small biomass model substrates. Phase equilibrium studies reveal that, under typical APR reaction conditions, the reaction mixture is in the liquid phase. Therefore, for the first time we show that the water-gas shift reaction (WGS), which is the second main reaction of APR, must be modeled in the liquid phase, resulting in an endothermic instead of an exothermic enthalpy of reaction. A significant implication of this finding is that, although APR has been introduced as more energy saving than conventional reforming methods, the WGS in APR has a comparable energy demand to the WGS in steam reforming (SR).
\end{abstract}

Adapted from:

Ripken, R. M.; Meuldijk, J.; Gardeniers, J. G. E.; Le Gac, S., Influence of the Water Phase State on the Thermodynamics of Aqueous-Phase Reforming for Hydrogen Production. Chemsuschem 2017, 10 (24), 4909-4913 


\subsection{Introduction}

As the world population and its energy demands continue to increase, and as conventional fossil fuel sources are depleting rapidly, research into alternative and renewable energy resources is gaining increasing attention. Hydrogen is both an attractive and clean energy vector: it has a high energy density and does not produce harmful products during combustion. ${ }^{1-3}$ Hydrogen can be produced from natural gas or coal, ${ }^{4-5}$ but these sources are neither renewable, nor are their processing methods sustainable. Using biomass waste streams, however, as a resource would valorize otherwise discarded materials. ${ }^{6-7}$ Current hydrogen production methods include liquefaction, pyrolysis, and steam reforming (SR). All these methods require temperatures above $473 \mathrm{~K}$ and, for liquefaction and pyrolysis only, pressures up to $200 \mathrm{bar}^{5, \text { 8-11 }^{-1}}$ In 2002 , AqueousPhase Reforming (APR) was introduced by the Dumesic research group as an alternative method to reform oxygenated carbohydrates with a $\mathrm{C}: \mathrm{O}$ ratio of $1: 1$ into $\mathrm{H}_{2}$ and $\mathrm{CO}_{2}$ using milder reaction conditions. ${ }^{12}$ Although many gaseous and liquid phase intermediates and products are formed by complicated and not yet fully understood reaction mechanisms, APR proceeds in two main reactions (Scheme 2.1). The substrate in the aqueous phase is first cracked into $\mathrm{CO}$ and $\mathrm{H}_{2}$. Next, $\mathrm{CO}$ is converted into $\mathrm{CO}_{2}$ in the water-gas shift reaction (WGS), while forming an additional amount of $\mathrm{H}_{2}$.

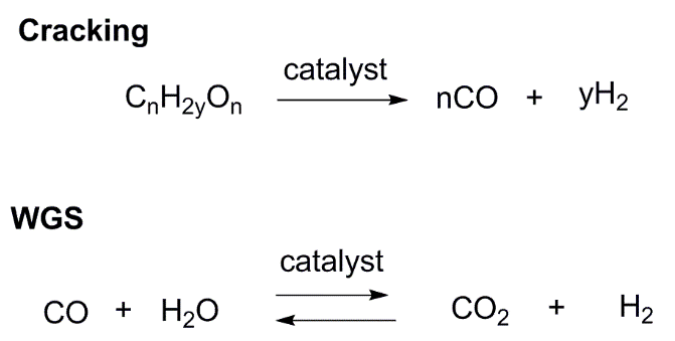

Scheme 2.1. Two main steps in Aqueous-Phase Reforming: the substrate is first cracked into $\mathrm{CO}$ and $\mathrm{H}_{2}$. Next, the formed $\mathrm{CO}$ is converted into $\mathrm{CO}_{2}$ and an additional amount of $\mathrm{H}_{2}$ is formed in the water-gas-shift (WGS).

To prevent evaporation of the reaction mixture, an elevated pressure must be applied, as determined by the vapor-liquid equilibrium (VLE) of the system. APR is typically performed at temperatures up to $550 \mathrm{~K}$ and pressures up to 55 bar. ${ }^{11}$ Previous thermodynamic studies have shown that the WGS is favorable for hydrogen production at temperatures approaching room temperature. ${ }^{13-14}$ Water has a dual role: it acts both as a solvent for the whole process and as a 
reactant for the second main reaction of APR. Here, we only considered water as a reactant in the WGS, unless specified otherwise.

Although the APR reaction conditions are a great improvement over the previously mentioned conventional hydrogen production methods, elevated temperatures and pressures are still needed. Herein, we evaluated the reaction thermodynamics of APR of a selection of low-molecular-mass substrates to explore the feasibility to perform APR closer to ambient conditions to make the process even more sustainable. In the ideal case, elevated temperatures and pressure would no longer be necessary to execute the process, saving a considerable amount of energy. The enthalpy and Gibbs free energy of reaction were evaluated as a function of the temperature at atmospheric pressure using data obtained from the literature and from the AspenPlus database. Next, to determine the lowest pressure required to maintain the reaction mixture in the liquid phase, the VLE and the saturated pressure $\left(P^{s a t}\right)$ were modeled as a function of both the temperature and the substrate mole fraction using AspenPlus.

\subsection{Materials and methods}

Phase studies were conducted using AspenPlus V8.4 software while applying the Redlich-KwongSoave equation of state with an alpha Boston-Mathias extrapolation (RKS-BM). Parameters were derived from the critical temperature and pressure of the components and a quadratic mixing rule was used. For the phase diagrams, a binary analysis was performed to obtain both Txy and Pxy graphs with varying water mass fraction. To study the influence of the substrate mole fraction, a sensitivity analysis was performed in which the temperature, pressure, and substrate mole fraction were varied in a Flash 2 separator. Thermodynamic trends for ethylene glycol, glycerol, xylose, and xylitol were calculated using the standard enthalpy of formation and the Gibbs free energy of formation data as reported in the literature. For these compounds, the specific heat capacity was maintained independent of the temperature while calculating the temperature-dependent enthalpy. The thermodynamics for the WGS was modelled in AspenPlus V8.4, which allowed inclusion of the temperature-dependent specific heat capacity. The temperature-dependent Gibbs free energy was obtained by applying the Gibbs-Helmholtz relation. 


\subsection{Results and discussion}

Ethylene glycol, glycerol, xylose, and xylitol were selected as biomass model substrates to study the APR reaction thermodynamics (Figure 2.1). Ethylene glycol and glycerol have been widely reported as APR substrates in the literature ${ }^{15-18}$ and act therefore as benchmark substrates. Xylose (the main component of hemicellulose) and xylitol were considered to study the use of hemicellulose derivatives as potential future APR feedstock. ${ }^{19}$

Although in APR many side reactions can take place, of which methanation is the most prominent, ${ }^{18,20}$ we limit our study to the two main reactions as presented in Scheme 2.1. Scheme 2.2 indicates if the enthalpy of these reactions is positive and if the reaction is therefore endothermic $(+\Delta)$ or if the enthalpy is negative and the reaction exothermic $(-\Delta)$ under standard conditions $(T=278.15 \mathrm{~K} ; 1$ bar $)$.

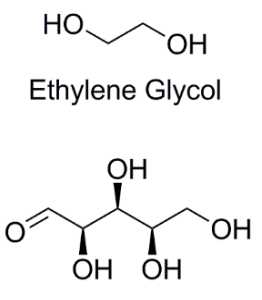

Xylose

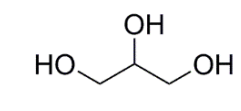

Glycerol

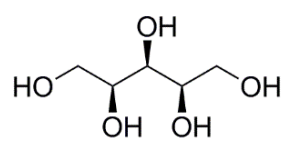

Xylitol

Figure 2.1. Biomass model substrates used in this study. Ethylene glycol and glycerol act as benchmark substrates, whereas xylose and xylitol were selected to study the potential of hemicellulose derivatives as APR feedstock.

\subsubsection{Phase studies}

As indicated in Scheme 2.2, the phase state of water during the WGS is of utmost importance, as it defines whether the WGS is an endothermic or an exothermic process. Furthermore, the energy released in the WGS with water in the gas liquid phase can be used for the cracking reaction, resulting in a lower total energy demand. Therefore, a thorough evaluation of the phase equilibrium of the model substrate/water solutions was performed to determine the phase of water under typical APR reaction conditions. For the small substrates studied here, solvation effects were not taken 


\section{Cracking}

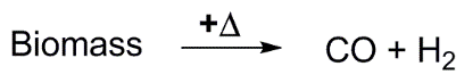

WGS (g)

$$
\begin{array}{lll}
\mathrm{CO}+\mathrm{H}_{2} \mathrm{O} \stackrel{-\Delta}{\longrightarrow} \mathrm{CO}_{2}+\mathrm{H}_{2} \\
\mathrm{CO}_{2}+\mathrm{H}_{2} \stackrel{+\Delta}{\longrightarrow} \mathrm{CO}+\mathrm{H}_{2} \mathrm{O}
\end{array}
$$

WGS (I)

$$
\begin{array}{lll}
\mathrm{CO}+\mathrm{H}_{2} \mathrm{O} \stackrel{+\Delta}{\longrightarrow} \mathrm{CO}_{2}+\mathrm{H}_{2} \\
\mathrm{CO}_{2}+\mathrm{H}_{2} \stackrel{-\Delta}{\longrightarrow} \mathrm{CO}+\mathrm{H}_{2} \mathrm{O}
\end{array}
$$

Scheme 2.2. Main APR reactions: cracking and water-gas-shift where water as a reagent is either in the gas (g) or liquid phase (1). The endothermic or exothermic character is indicated by $+\Delta$ and $-\Delta$ respectively.

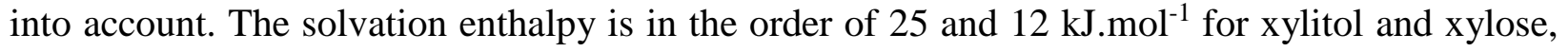
respectively, which is negligible compared to the overall reaction enthalpy for APR. ${ }^{21-22}$

Phase equilibrium studies were conducted for pressures of 1, 22, 30, and 56 bar and temperatures of 298, 498, 538, and $623 \mathrm{~K}$. These APR operating conditions are in accordance with those reported in the literature, as summarized in Table 2.1, for the substrates considered in this study. Xylose could not be included in this table as, to the best of our knowledge, it has not been used yet as an APR substrate in experimental work. Glucose was added to the table instead to show typical reaction conditions used for APR of sugars.

The Txy and Pxy VLE-diagrams were obtained using AspenPlus with a Redlich-Kwong-Soave Boston-Mathias (RKS-BM) model (see Appendix, A2.1). This model allows calculating the heat duty and is recommended for hydrocarbon processing by AspenPlus. Furthermore, this model has been successfully applied to study biomass reforming, including the reforming of polar compounds. ${ }^{23-24}$ The VLE-diagrams, indicate that the substrate/ $\mathrm{H}_{2} \mathrm{O}$ binary mixtures are in the liquid phase under APR reaction conditions. In these calculations the mole fraction of the model substrate in water could not be accounted for, even though it can influence the phase equilibrium significantly. Therefore, the saturated-vapor pressure as a function of both the temperature and the 
Table 2.1. APR operating conditions reported in the literature and phase state of water at these conditions determined using the VLE-diagrams or the saturated vapor pressure $P^{s a t}$ at $T=498 \mathrm{~K}$ and a substrate mole fraction of 0.2 .

\begin{tabular}{llcccc}
\hline & & Ethylene Glycol & Glycerol & Xylitol & Glucose \\
\hline Literature & Reference & Cortright $^{12}$ & Cortright $^{12}$ & Kirilin $^{19}$ & Cortright $^{12}$ \\
& $T[\mathrm{~K}]$ & 498 & 498 & 498 & 538 \\
& $P_{\text {applied }}[\mathrm{bar}]$ & 29 & 29 & 29.3 & 56 \\
Calculated & VLE- & Liquid & Liquid & Liquid & - \\
phase state & diagrams & & & & \\
& $P^{\text {sat }}[\mathrm{bar}]$ & 21.9 & 21.1 & 22.4 & - \\
\hline
\end{tabular}

mole fraction was also evaluated using the same software and model while performing a sensitivity analysis. The results obtained from these calculations confirm that the reaction mixture is in the liquid phase state under APR process conditions (see Appendices, A2.2). The applied pressure is higher than the calculated vapor pressure at the operating temperature for a substrate mole fraction of 0.2. Altogether, the substrate $/ \mathrm{H}_{2} \mathrm{O}$ mixture is entirely in the liquid phase under APR. Therefore, the WGS should be considered in the liquid phase instead of the gas phase as currently reported in the literature. ${ }^{11,20}$ To the best of our knowledge, this is the first time that a study of the reaction thermodynamics of the WGS is performed with water in the liquid phase, resulting in an endothermic process.

\subsubsection{Reaction thermodynamics}

To prove the endothermic character of the WGS in the liquid phase and to evaluate the reaction thermodynamics, the enthalpy and the Gibbs free energy of reaction were calculated from both the enthalpy of formation and Gibbs free energy of formation under standard conditions. Equation $2.1^{25}$ was derived for the temperature-dependent enthalpy of reaction (Appendices, A2.3.3):

$$
d H=c_{p} d T+[1-\alpha T] V d P
$$

Where $H$ is the enthalpy or reaction, $c_{p}$ the specific heat capacity, $\alpha$ the thermal expansion coefficient, and $T, V$ and $P$ the temperature, volume and pressure, respectively. 
The gases formed during APR $\left(\mathrm{CO}, \mathrm{CO}_{2}, \mathrm{H}_{2}\right)$ were considered as ideal. Therefore, the pressure dependency of the enthalpy was neglected. The non-ideal gas behavior is expected to have only a small influence on the reaction enthalpy. Both the reaction enthalpy and the Gibbs free energy of reaction were calculated at atmospheric pressure. At 1 bar phase transitions occur while increasing the temperature, which was included in the reaction thermodynamics. In this way, the difference in energy between the liquid phase and the gas phase reaction is more clearly illustrated. Furthermore, our end goal was to explore APR at ambient conditions, which implies to conduct the reaction at atmospheric pressure. The phase transition temperature of the substrates was chosen as the upper temperature limit. The specific heat capacity $\left(c_{p}\right)$ values for the model substrates were kept constant for the temperature range studied, as to the best of our knowledge, the values of $c_{p}(T)$ for these temperatures are not available. For the compounds involved in the WGS, the temperature dependency of the specific heat capacity was accounted for. The Gibbs free energy of reaction as a function of the temperature was calculated using the Gibbs-Helmholtz relation. The thermodynamic constants for the substrates were collected from several sources, whereas only one source was used for each substrate (Table 2.2). Unfortunately, no data was found for the Gibbs free energy of xylitol. The temperature-dependent enthalpy values for $\mathrm{CO}, \mathrm{H}_{2} \mathrm{O}, \mathrm{CO}_{2}$, and $\mathrm{H}_{2}$ were obtained from AspenPlus by applying the RKS-BM model. Although only one source was used to acquire data for a single substrate, one should be cautious when comparing the results for different substrates, as there could be inconsistencies between the different sources.

Table 2.2. Thermodynamic data for ethylene glycol, glycerol, xylose and xylitol. The phase of the reactants at $T=298.15 \mathrm{~K}$, liquid (1) or solid (s) is indicated.

\begin{tabular}{lcccc}
\hline Thermodynamic value & $\begin{array}{c}\text { Ethylene Glycol } \\
\text { (l) }\end{array}$ & $\begin{array}{c}\text { Glycerol } \\
\text { (l) }\end{array}$ & $\begin{array}{c}\text { Xylose } \\
\text { (s) }\end{array}$ & $\begin{array}{c}\text { Xylitol } \\
\text { (s) }\end{array}$ \\
\hline Reference & Murphy $^{26}$ & Verevkin $^{27}$ & Goldberg $^{22}$ & AspenPlus \\
$\begin{array}{l}\text { Standard enthalpy of } \\
\text { formation [kJ.mol }\end{array}{ }^{-1}$ of & -451.5 & -669.3 & -1057.84 & -1118.5 \\
$\begin{array}{l}\text { Standard Gibbs free energy } \\
\left.\text { of formation [kJ.mol }{ }^{-1}\right]\end{array}$ & -319.6 & -478.3 & -744.59 & - \\
$\begin{array}{l}\text { Specific heat capacity } \\
{\left[\mathrm{J}_{\mathrm{mol}}{ }^{-1} \mathrm{~K}^{-1} \text { ] }\right.}\end{array}$ & 149.8 & 218.9 & 184 & - \\
\hline
\end{tabular}




\subsubsection{Enthalpy and Gibbs free energy of reaction}

Figures 2.2 and 2.3 show the temperature-dependent enthalpy and the Gibbs free energy of reaction for APR of all four biomass model substrates and for the WGS. To establish these graphs, only the water molecules that are acting as a reactant were included in the calculations. These results are in accordance with data previously published for the APR of ethylene glycol and the WGS (Appendix A2.4). ${ }^{20}$ The sharp drop in the reaction enthalpy is indicative for the heat of evaporation of the reacting water molecules. In the liquid phase, both the cracking and the WGS are endothermic, whereas at atmospheric pressure and temperatures above the boiling point of water, the WGS is exothermic. As the enthalpy does not change much within the temperature range studied, reforming in the liquid phase seems to be highly unfavorable. Furthermore, the reaction is exergonic at temperatures above $310 \mathrm{~K}$ not only for the APR of all substrates, but also for the WGS. The highly positive enthalpy and the negative Gibbs free energy indicate that the entropy has to increase to such an extent that it compensates for the high, positive enthalpy. A gain in entropy can also explain the decreasing Gibbs free energy for higher temperatures. Moreover, a higher temperature would not only be beneficial for the entropy, but it would also increase the rate

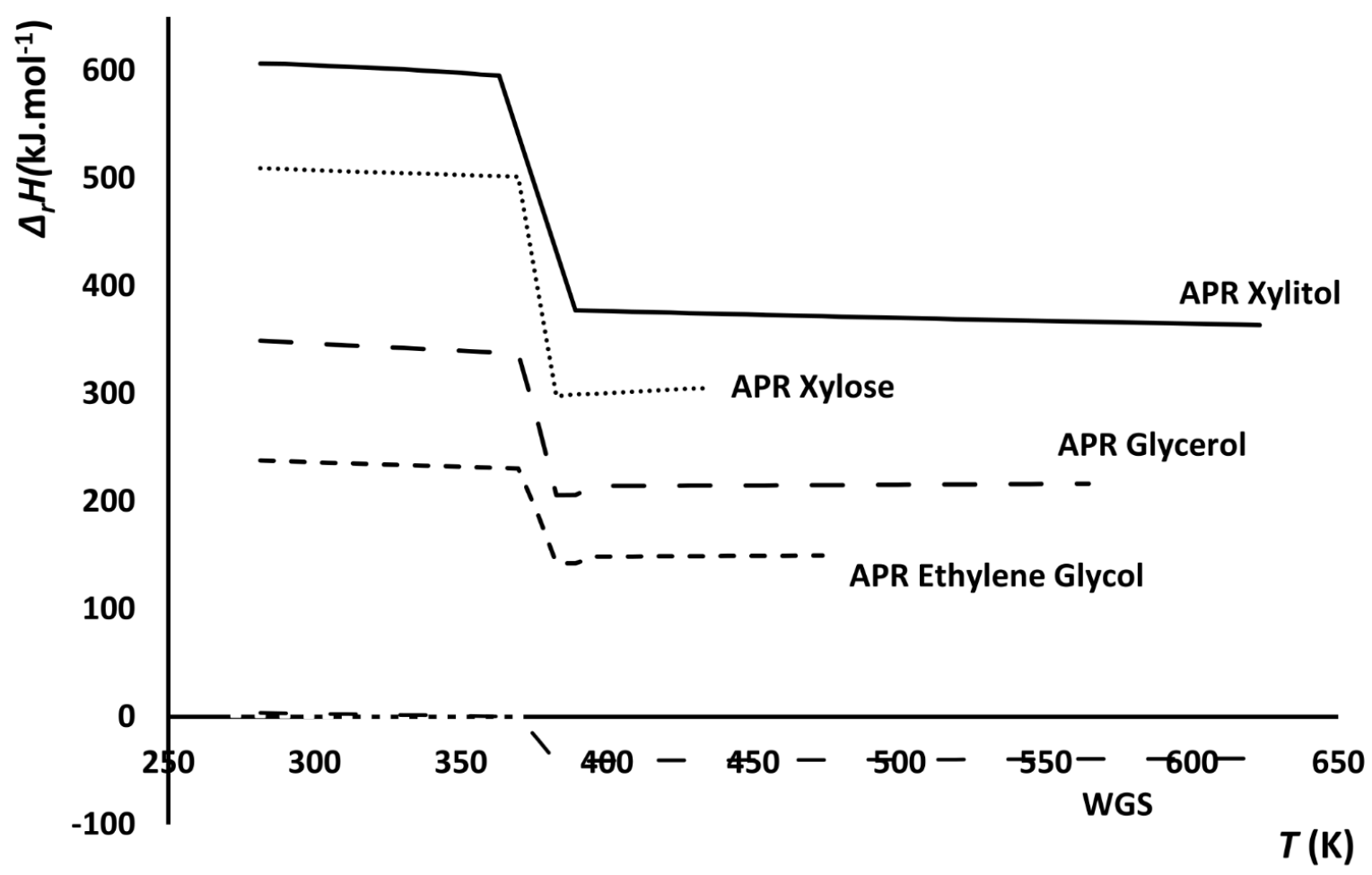

Figure 2.2. Enthalpy of reaction for APR and the WGS as a function of the temperature at standard pressure. 


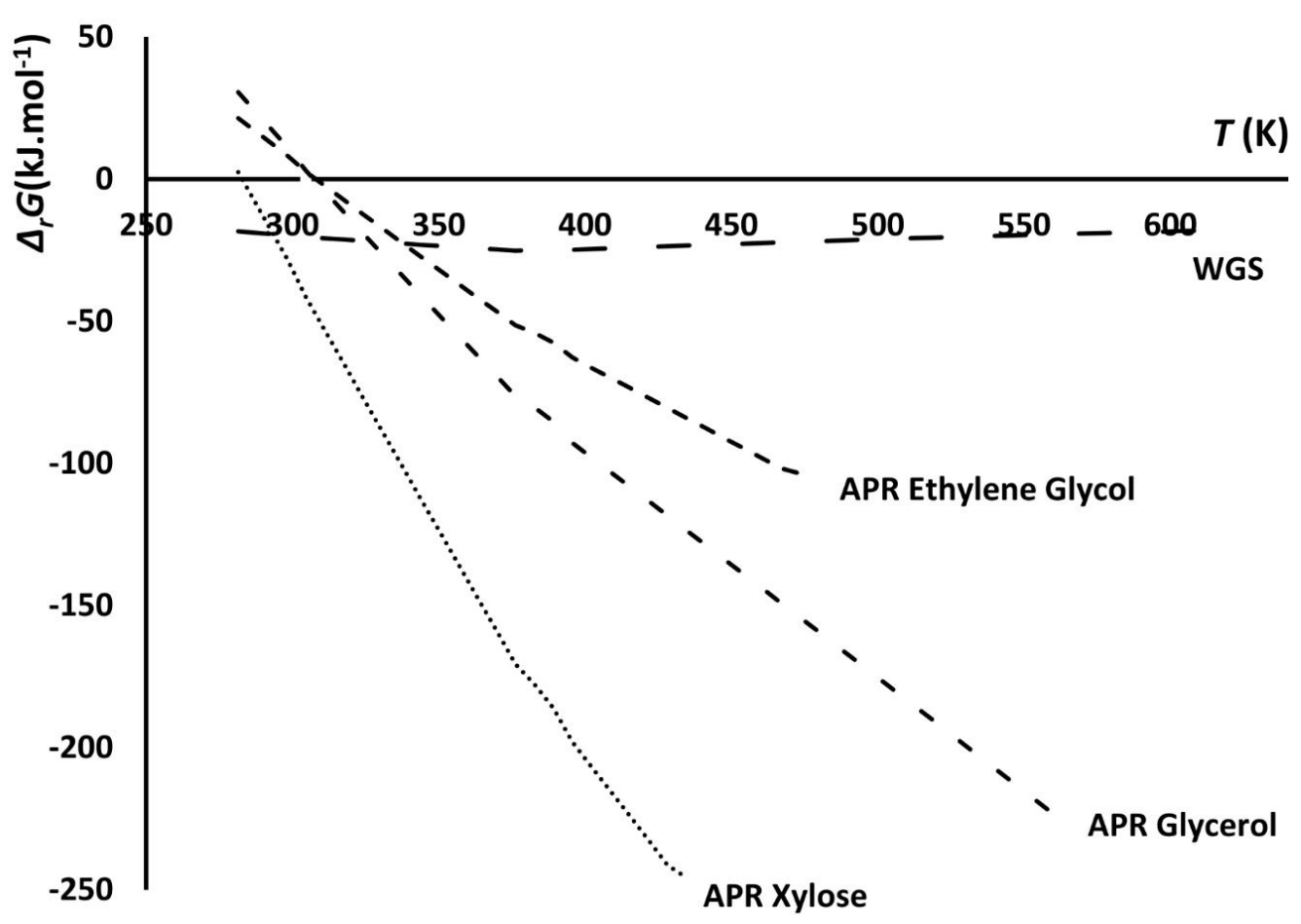

Figure 2.3. Gibbs free energy of reaction for APR and the WGS as a function of the temperature at standard pressure.

of reaction. The differences between the substrates can be explained in terms of carbon number. A higher carbon number means a larger APR enthalpy, as more chemical bonds need to be broken. As a result, more $\mathrm{H}_{2}$ and $\mathrm{CO}_{2}$ are formed, and a favorable entropy is found for larger substrates. Therefore, APR of larger biomass substrates is preferred in terms of Gibbs free energy.

\subsubsection{Discussion}

First, based on the calculated Gibbs free energy, APR can be performed at a lower temperature starting from $310 \mathrm{~K}$. The literature APR reaction conditions summarized in Table 2.1 are all above this minimum temperature needed to perform APR. Similarly, at this temperature, no external pressure has to be applied to maintain the system in the liquid phase state. Still, the process is expected to run more efficiently at a higher temperature as a result of the increased entropy and a more negative Gibbs free energy. However, it is unlikely that thermodynamic equilibrium is reached in an experiment. Kinetic parameters such as conversion, reaction rate, and selectivity also 
depend on the pressure and temperature, which affect the hydrogen yield. Applying a higher pressure might still be beneficial for kinetic reasons, even when it is not required from a thermodynamic point of view: This would be the case when the volume of activation for the specific reaction is positive. Therefore, an additional thorough analysis of the kinetics is also essential to eventually optimize the reaction parameters to maximize hydrogen production. This kinetic study is however beyond the scope of the present work.

Furthermore, based on these findings, the WGS in the liquid phase is not as energetically favorable as in the gas phase, which is the phase in which the WGS is currently considered to take place in both APR and SR. This is particularly the case when the reaction enthalpy and Gibbs free energy of reaction are considered. When the energy demand of the WGS in SR is calculated from steam and gaseous $\mathrm{CO}$, the enthalpy of reaction is lower than for APR as a result of the exothermic WGS in SR. However, in SR and in the gas-phase WGS, the reacting water molecules have to be vaporized first and this heat of evaporation has to be added to the energy balance. The heat of evaporation of water, as reactant, is exactly the same as the energy later gained in the exothermic WGS. In other words, the reaction energy demand for the WGS in APR and SR is the same (Figure 2.4).

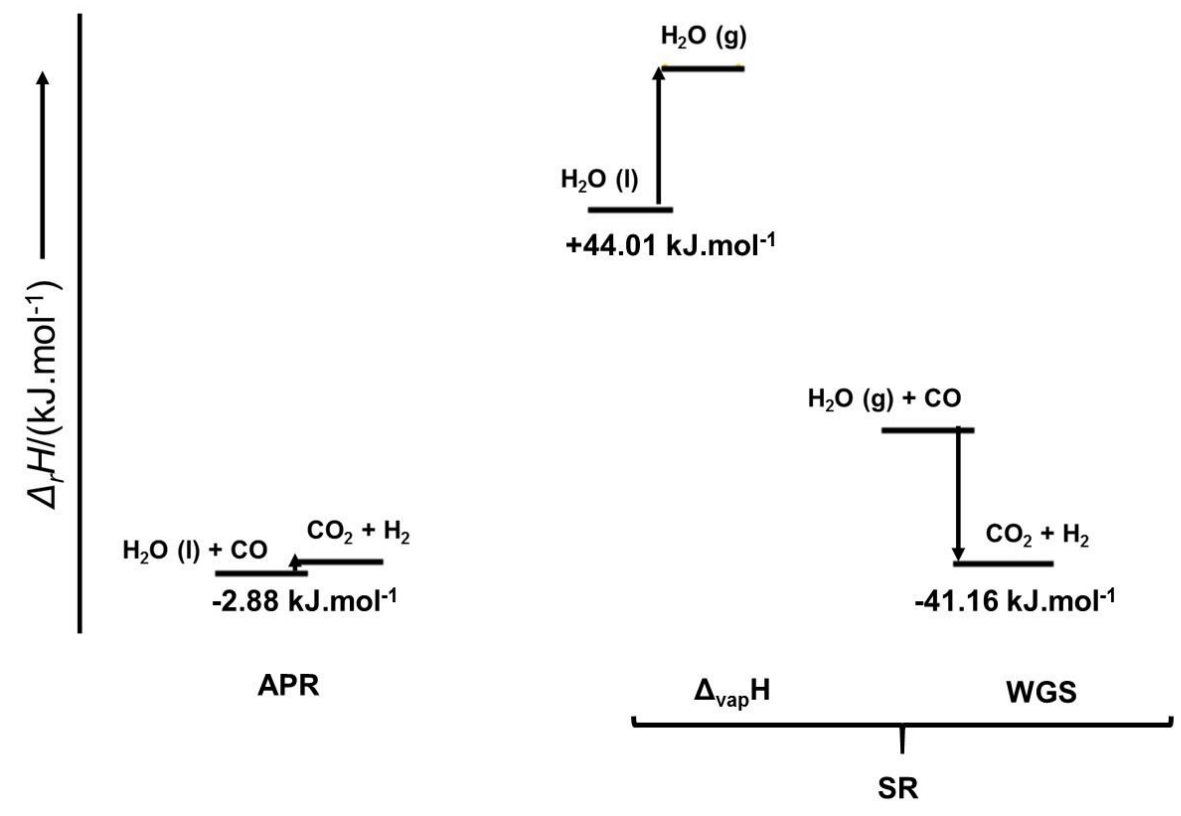

Figure 2.4. Schematic comparison of the WGS reaction enthalpy between APR and SR when including the heat of evaporation of water as a solvent. 
There is still one significant difference between the two reforming approaches; the state of the reaction mixture. For SR, the reaction mixture must be vaporized, including water that is acting as solvent, which has not been included in this study. This additional energy requirement for the evaporation of water as a solvent results in an overall higher energy demand for SR than for APR.

Finally, we propose that the phase state of the reactants would have implications for the reaction mechanism. Both SR and APR comprise the same main reactions: cracking of the substrate followed by the WGS (Scheme 2.1), but the phase state of the reactants is different for SR and APR. In the case of SR, all the reactants are in the gas phase and both the cracking and the WGS are two-phase reactions between the gaseous compounds and the heterogeneous catalyst. In APR, the cracking reaction is again a two-phase reaction, but this time between the substrate in the aqueous phase and the solid catalyst. The WGS, however, is a three-phase reaction, as concluded from our thermodynamic study, with water as a reactant in the liquid phase, $\mathrm{CO}$ in the gas phase, and the solid catalyst. Therefore, water has to come in contact with both the solid catalyst and the gaseous $\mathrm{CO}$ and the reaction takes place at the gas/liquid/solid interface (Figure 2.5). Further research is still however needed to clarify the exact reaction mechanism of the WGS.

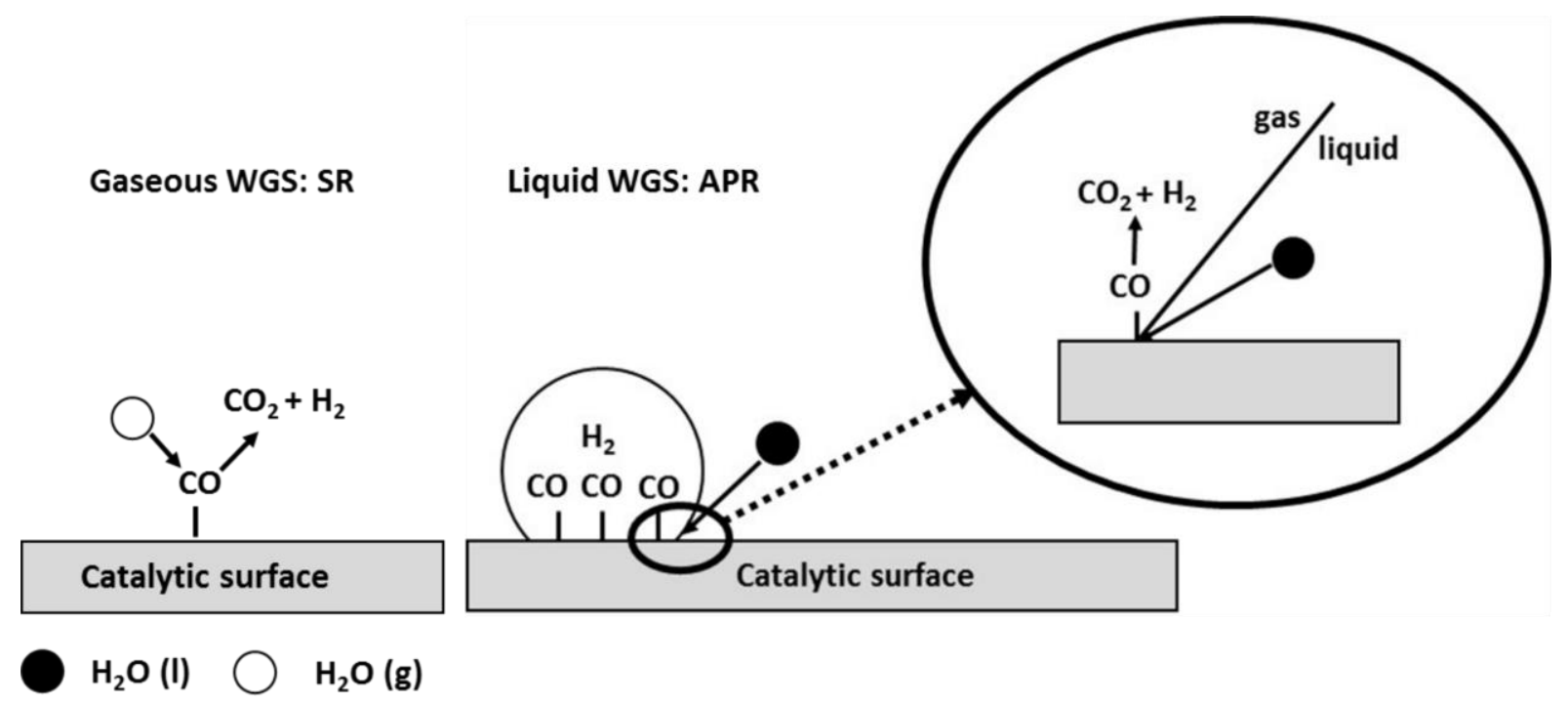

Figure 2.5. Schematic interpretations of the WGS reaction mechanisms for SR and APR showing the phase state of the reactants. For SR, the WGS is a two-phase process as all compounds are in the gas phase and a heterogeneous catalyst is used. However, in APR water in the liquid phase reacts at the solid/gas/liquid interface with $\mathrm{CO}$ in the gas phase present in bubbles formed during the cracking step. 


\subsection{Conclusion}

Here, the reaction thermodynamics for aqueous-phase reforming (APR) of ethylene glycol, glycerol, xylose, and xylitol was studied to investigate the possibility of performing APR closer to ambient conditions. Based only on thermodynamic considerations, APR of the studied substrates could be performed at a temperature as low as $310 \mathrm{~K}$ without additional external pressure. For the first time, we have demonstrated that the water-gas shift reaction (WGS) should be modeled in the liquid phase when calculating the reaction enthalpy and the Gibbs free energy for APR. The enthalpy shows little variation with the temperature and the WGS proves to be endothermic in the liquid state, which, at first sight, makes APR thermodynamically unfavorable compared to steam reforming (SR). However, when including the heat of evaporation of water as a reagent to calculate the energy demand of the WGS in SR, the energy requirement of this reaction in APR and SR is exactly the same. The phase state of water as a reactant also has implications for the mechanism of the WGS; the WGS in SR is a two-phase reaction, compared to a reaction at a gas-liquid-solid interface in APR.

\section{Acknowledgements}

The authors thank Prof. Dr. P.C.A. Bruijnincx from Utrecht University, The Netherlands, for the fruitful discussions.

\section{References}

1. He, T.; Pachfule, P.; Wu, H.; Xu, Q.; Chen, P., Hydrogen carriers. Nature Reviews Materials 2016, 1 (12).

2. Balat, H.; Kirtay, E., Hydrogen from biomass - Present scenario and future prospects. Int. J. Hydrogen Energy 2010, 35 (14), 7416-7426.

3. Ni, M.; Leung, D. Y. C.; Leung, M. K. H.; Sumathy, K., An overview of hydrogen production from biomass. Fuel Process. Technol. 2006, 87 (5), 461-472. 
4. Chen, G.-y.; Li, W.-q.; Chen, H.; Yan, B.-b., Progress in the aqueous-phase reforming of different biomass-derived alcohols for hydrogen production. Journal of Zhejiang UniversitySCIENCE A 2015, 16 (6), 491-506.

5. Udengaard, N. R., Hydrogen production by steam reforming of hydrocarbons. Abstracts of Papers of the American Chemical Society 2004, 228, U682-U682.

6. Navarro, R. M.; Sánchez-Sánchez, M. C.; Alvarez-Galvan, M. C.; Valle, F. d.; Fierro, J. L. G., Hydrogen production from renewable sources: biomass and photocatalytic opportunities. Energy \& Environmental Science 2009, 2 (1), 35-54.

7. Tanksale, A.; Beltramini, J. N.; Lu, G. M., A review of catalytic hydrogen production processes from biomass. Renewable \& Sustainable Energy Reviews 2010, 14 (1), 166-182.

8. Holladay, J. D.; Hu, J.; King, D. L.; Wang, Y., An overview of hydrogen production technologies. Catal. Today 2009, 139 (4), 244-260.

9. Huber, G. W.; Dumesic, J. A., An overview of aqueous-phase catalytic processes for production of hydrogen and alkanes in a biorefinery. Catal. Today 2006, 111 (1), 119-132.

10. Elliott, D. C., Catalytic hydrothermal gasification of biomass. Biofuels Bioproducts \& Biorefining-Biofpr 2008, 2 (3), 254-265.

11. Chheda, J. N.; Huber, G. W.; Dumesic, J. A., Liquid-phase catalytic processing of biomassderived oxygenated hydrocarbons to fuels and chemicals. Angewandte Chemie-International Edition 2007, 46 (38), 7164-7183.

12. Cortright, R. D.; Davda, R. R.; Dumesic, J. A., Hydrogen from catalytic reforming of biomass-derived hydrocarbons in liquid water. Nature 2002, 418 (6901), 964-967.

13. Giwa, A.; Raji, Y. O.; Giwa, S. O., Water-Gas Shift Process for Hydrogen Production Effects of Operating Parameters. IJSER 2014, (5), 1474-1483.

14. Demirel, E.; Azcan, N.; Iaeng, Thermodynamic Modeling of Water-Gas Shift Reaction in Supercritical Water. In World Congress on Engineering and Computer Science, Wcecs 2012, Vol Ii, 2012; pp 804-807.

15. Vaidya, P. D.; Rodrigues, A. E., Glycerol Reforming for Hydrogen Production: A Review. Chemical Engineering \& Technology 2009, 32 (10), 1463-1469.

16. Seretis, A.; Tsiakaras, P., Aqueous phase reforming (APR) of glycerol over platinum supported on Al2O3 catalyst. Renewable Energy 2016, 85, 1116-1126. 
17. Guo, Y.; Liu, X. H.; Azmat, M. U.; Xu, W. J.; Ren, J. W.; Wang, Y. Q.; Lu, G. Z., Hydrogen production by aqueous-phase reforming of glycerol over Ni-B catalysts. Int. J. Hydrogen Energy 2012, 37 (1), 227-234.

18. Davda, R. R.; Shabaker, J. W.; Huber, G. W.; Cortright, R. D.; Dumesic, J. A., Aqueousphase reforming of ethylene glycol on silica-supported metal catalysts. Applied Catalysis B: Environmental 2003, 43 (1), 13-26.

19. Kirilin, A. V.; Tokarev, A. V.; Kustov, L. M.; Salmi, T.; Mikkola, J. P.; Murzin, D. Y., Aqueous phase reforming of xylitol and sorbitol: Comparison and influence of substrate structure. Applied Catalysis a-General 2012, 435, 172-180.

20. Davda, R. R.; Shabaker, J. W.; Huber, G. W.; Cortright, R. D.; Dumesic, J. A., A review of catalytic issues and process conditions for renewable hydrogen and alkanes by aqueous-phase reforming of oxygenated hydrocarbons over supported metal catalysts. Applied Catalysis BEnvironmental 2005, 56 (1-2), 171-186.

21. Ali, A.; Bidhuri, P., Solvation thermodynamics of xylitol in water and in aqueous amino acids at 298.15 K. J. Phys. Org. Chem. 2013, 26 (1), 54-58.

22. Goldberg, R. N.; Tewari, Y. B., THERMODYNAMIC AND TRANSPORTPROPERTIES OF CARBOHYDRATES AND THEIR MONOPHOSPHATES - THE PENTOSES AND HEXOSES. J. Phys. Chem. Ref. Data 1989, 18 (2), 809-880.

23. Paviet, F.; Chazarenc, F.; Tazerout, M., Thermo Chemical Equilibrium Modelling of a Biomass Gasifying Process Using ASPEN PLUS. International Journal of Chemical Reactor Engineering 2009, 7.

24. De Kam, M. J.; Morey, R. V.; Tiffany, D. G., Biomass Integrated Gasification Combined Cycle for heat and power at ethanol plants. Energy Convers. Manage. 2009, 50 (7), 1682-1690.

25. Enthalpy.

26. Murphy, R. M., Introduction to chemical processes: principles, analysis, synthesis. Mc Graw-Hill: 2007.

27. Verevkin, S. P.; Zaitsau, D. H.; Emel'yanenko, V. N.; Zhabina, A. A., Thermodynamic properties of glycerol: Experimental and theoretical study. Fluid Phase Equilib. 2015, 397, 87-94. 


\section{A2.1. Vapor-liquid-equilibrium}

The VLE-diagrams were obtained by AspenPlus V8.4 using the binary analysis tool with a RKS-

BM model. Both the Txy and the Pxy-graphs were investigated for each of the four model substrates, ethylene glycol, glycerol, xylose and xylitol, when dissolved in water. Typical APR reaction conditions are indicated in the graphs. All substrate-water mixtures are present in the liquid phase at the APR reaction conditions.

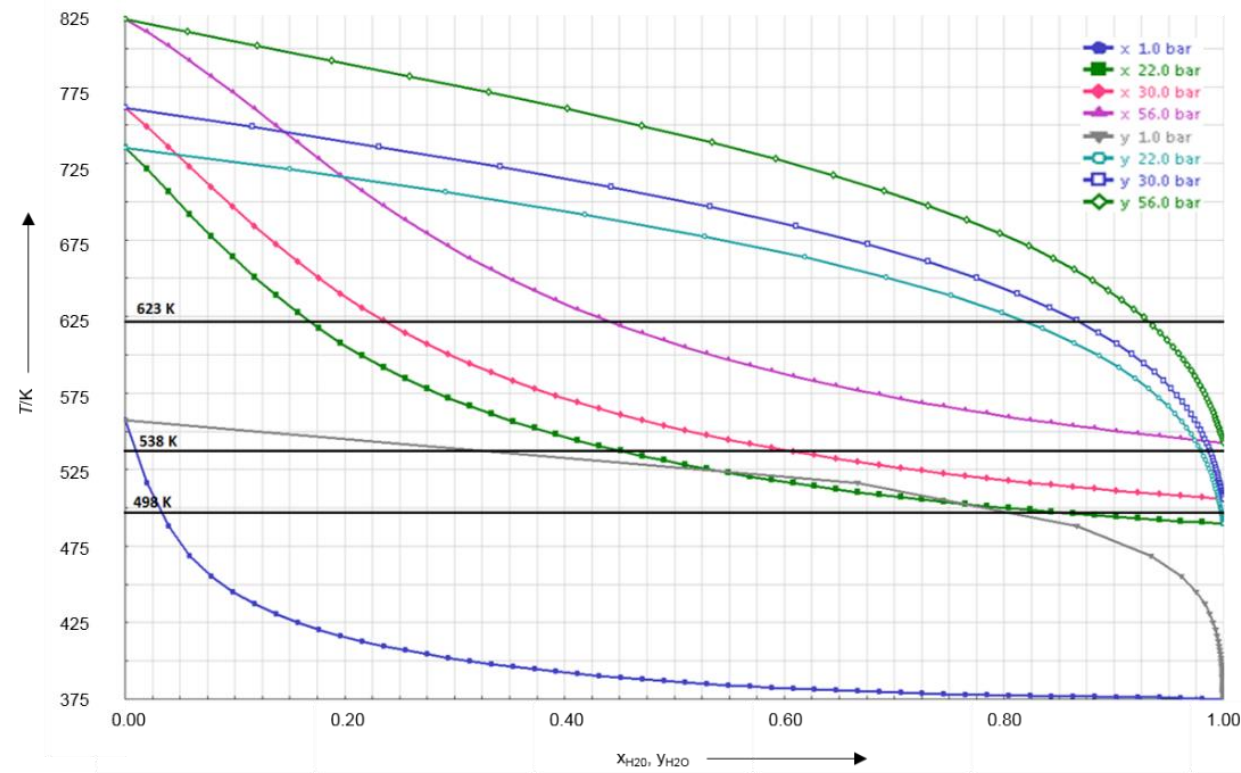

Figure A2.1.1. Txy-diagram glycerol

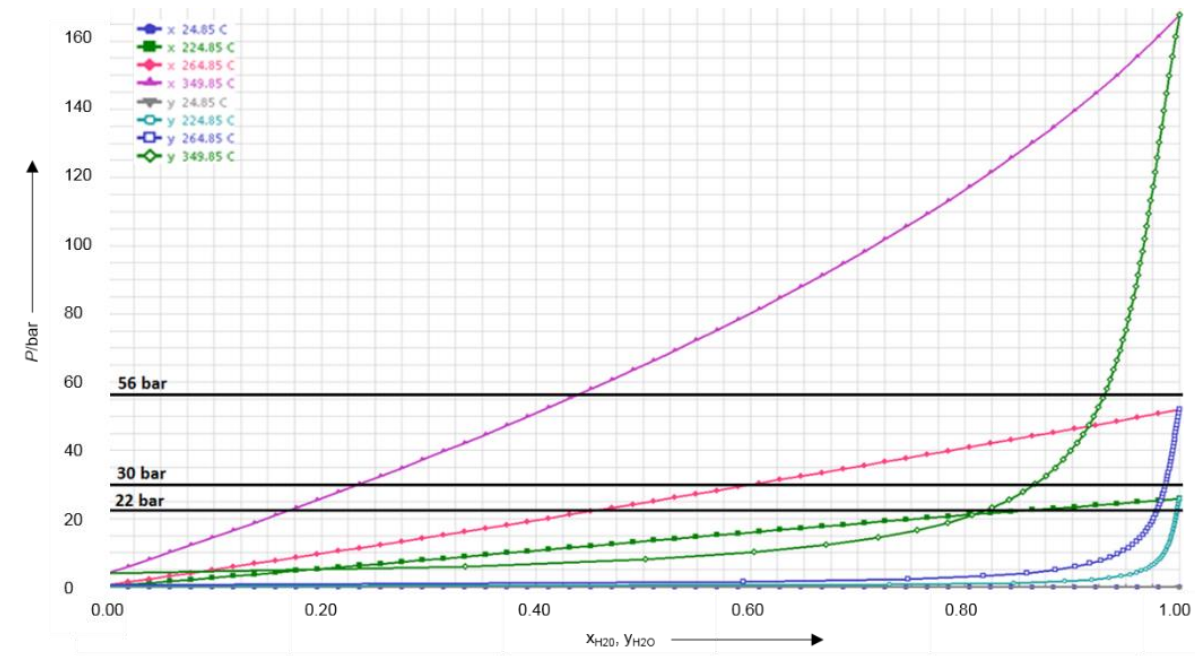

Figure A2.1.2. Pxy-diagram glycerol 


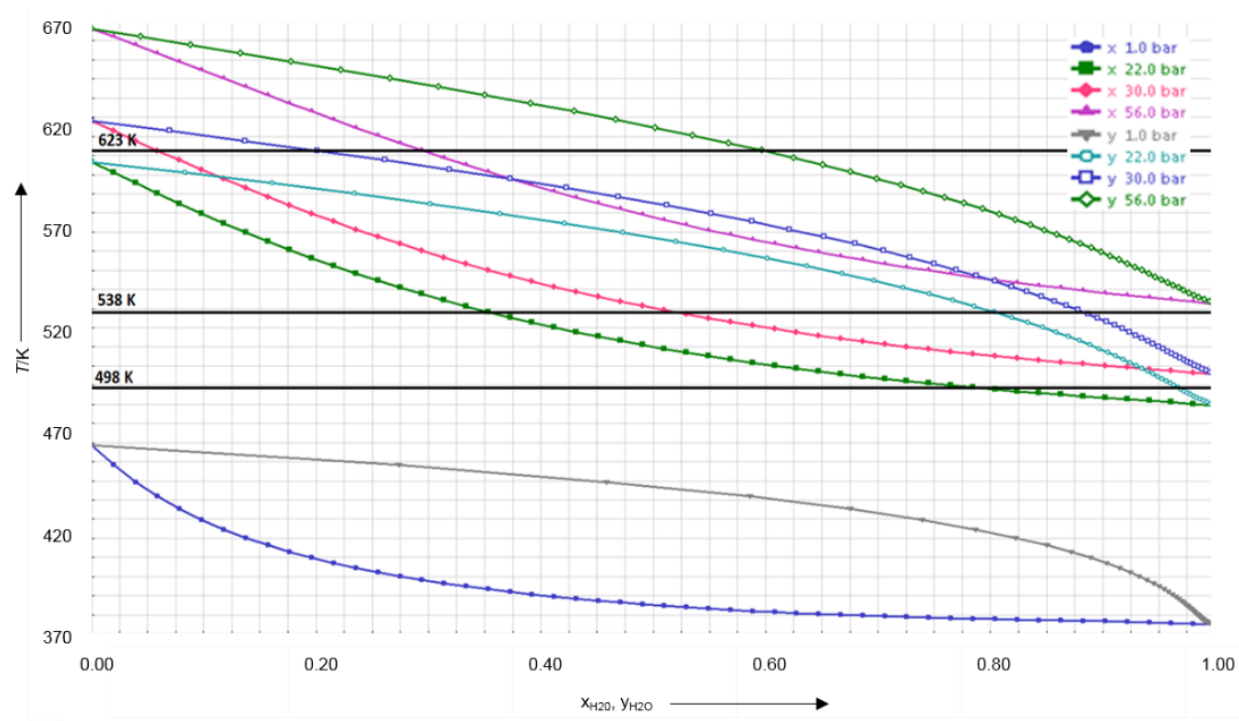

Figure A2.1.3. $T x y$-diagram ethylene glycol

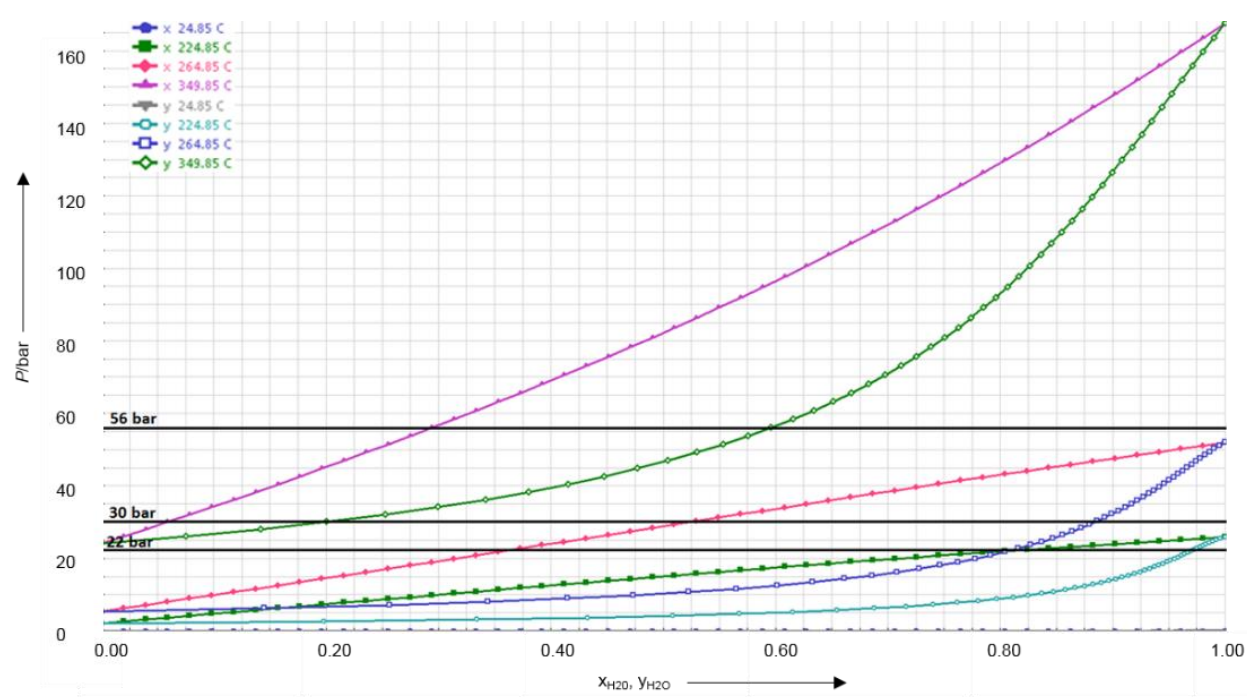

Figure A2.1.4. Pxy-diagram ethylene glycol 


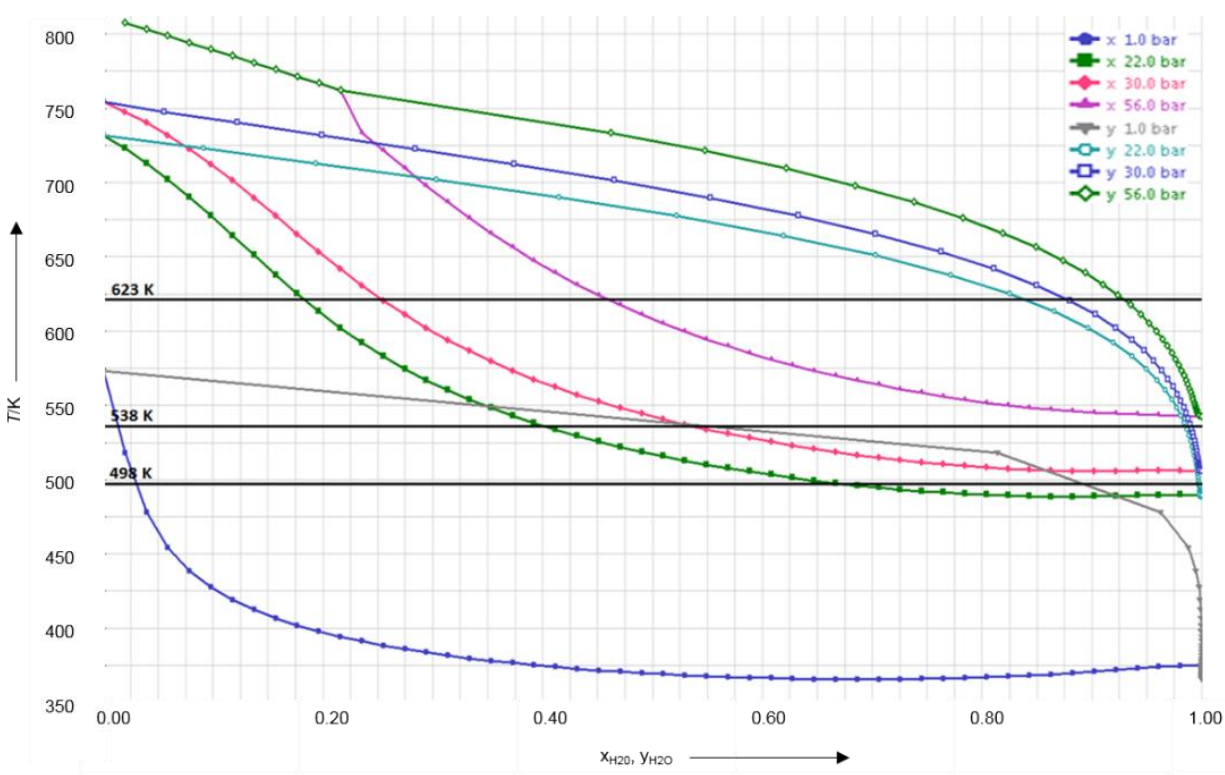

Figure A2.1.5. Txy-diagram xylose

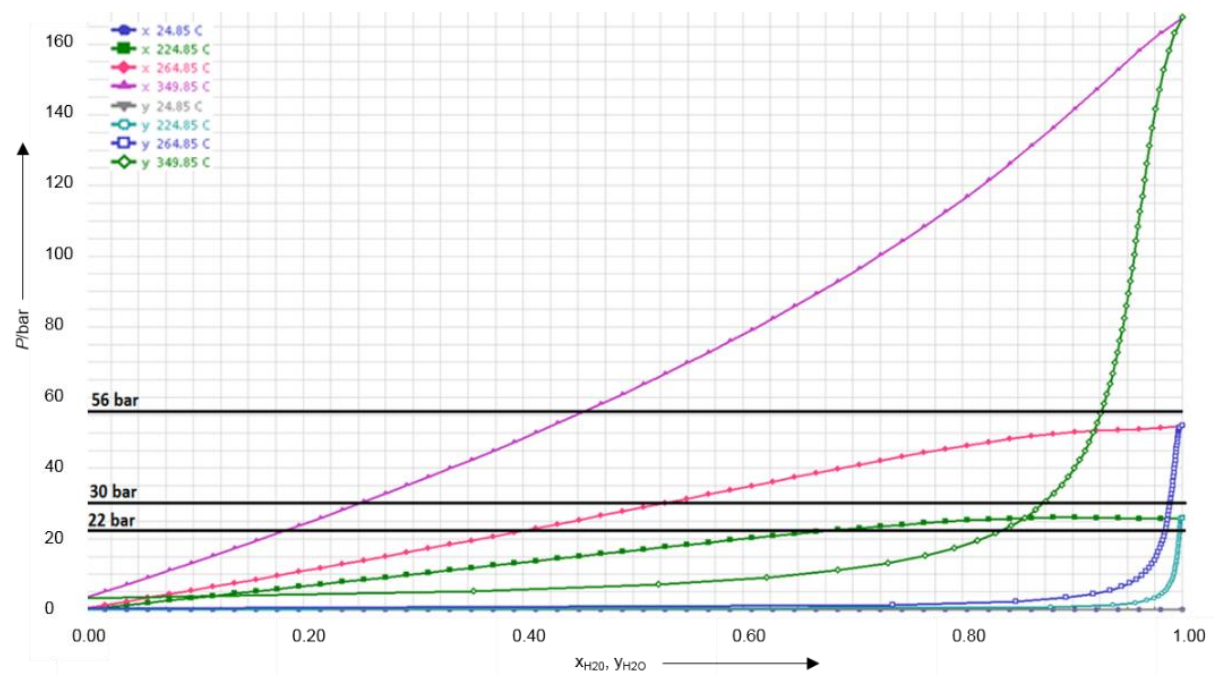

Figure A2.1.6. Pxy-diagram xylose 


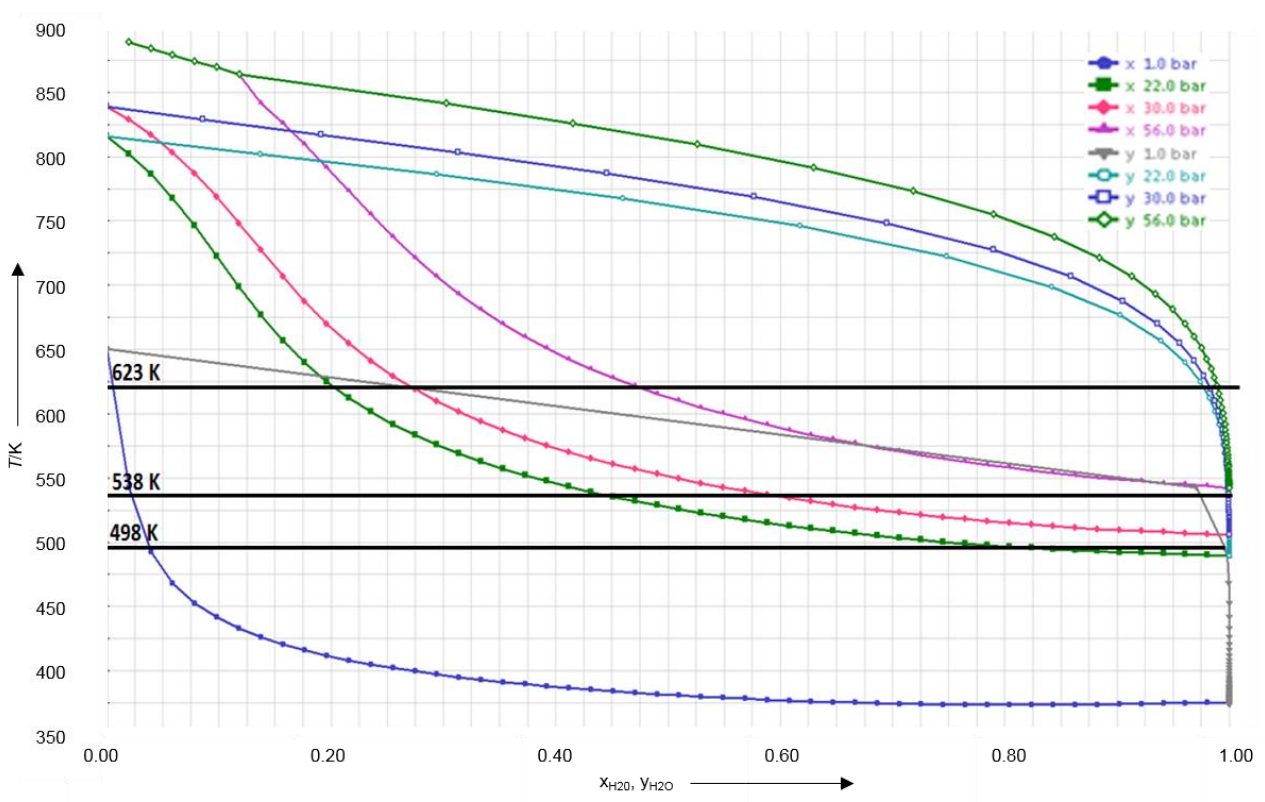

Figure A2.1.7. $T x y$-diagram xylitol

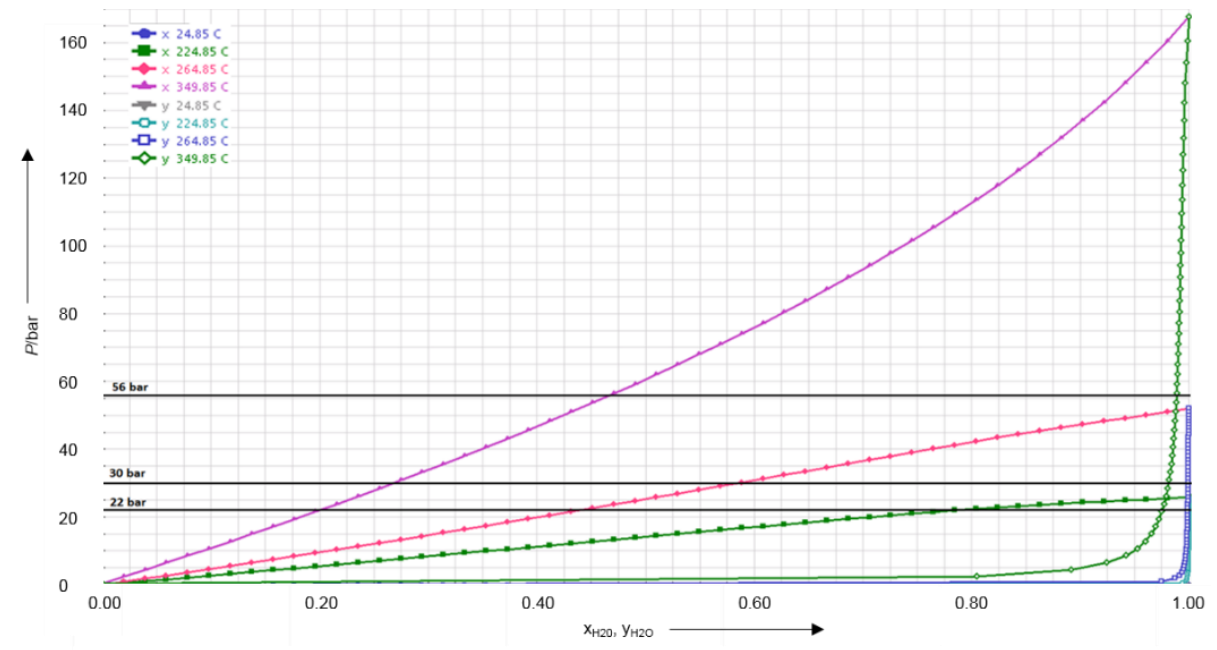

Figure A2.1.8. Pxy-diagram xylitol 


\section{A2.2. Saturated vapor pressure}

To investigate the phase equilibria while including the mole fraction of the substrate in the substrate-water mixture, a sensitivity analysis was performed in AspenPlus V8.4 with a Flash 2 separator and a RKS-BM model. The resulting saturated vapor pressure graphs as a function of the temperature confirm the findings from the VLE-diagrams that the reaction mixture is in the liquid phase at APR operating conditions.

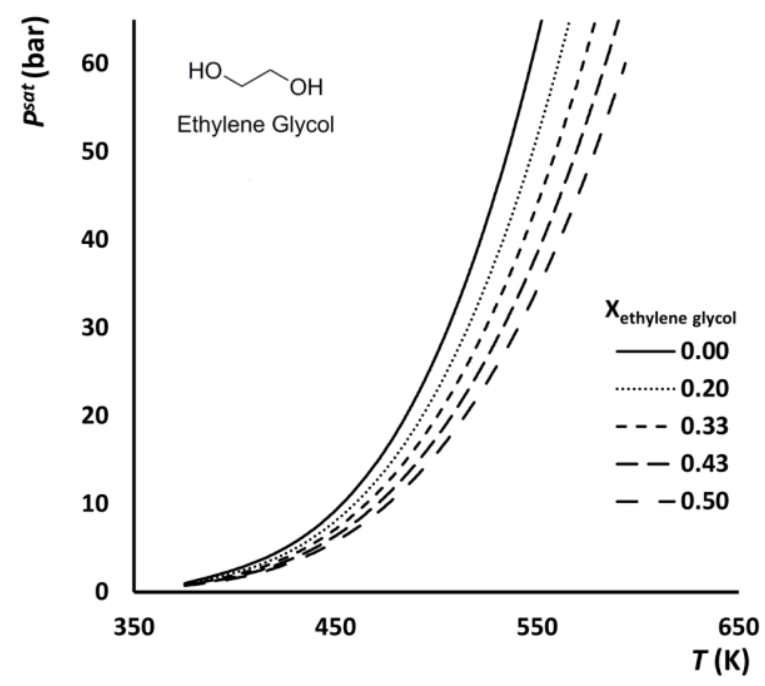

Figure A2.2.1 Saturated vapor pressure ethylene glycol

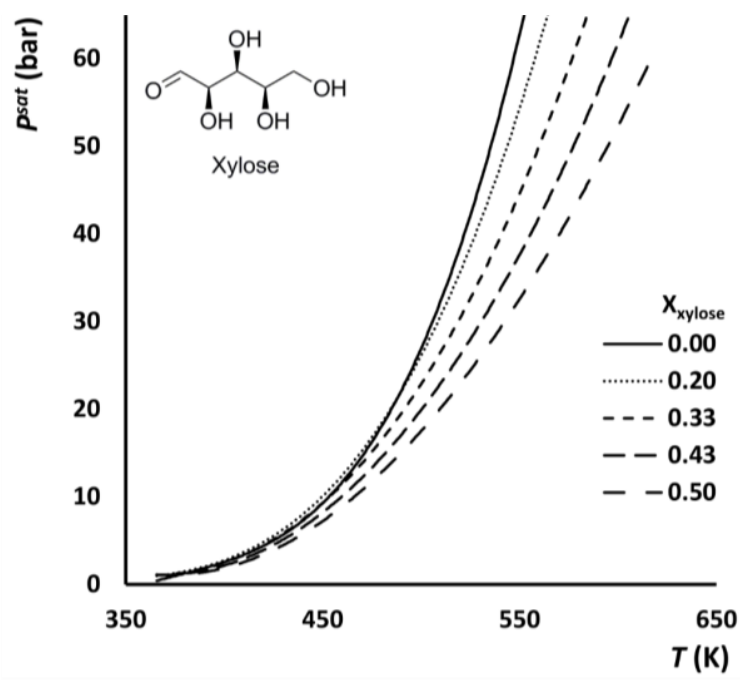

Figure A2.2.3 Saturated vapor pressure xylose

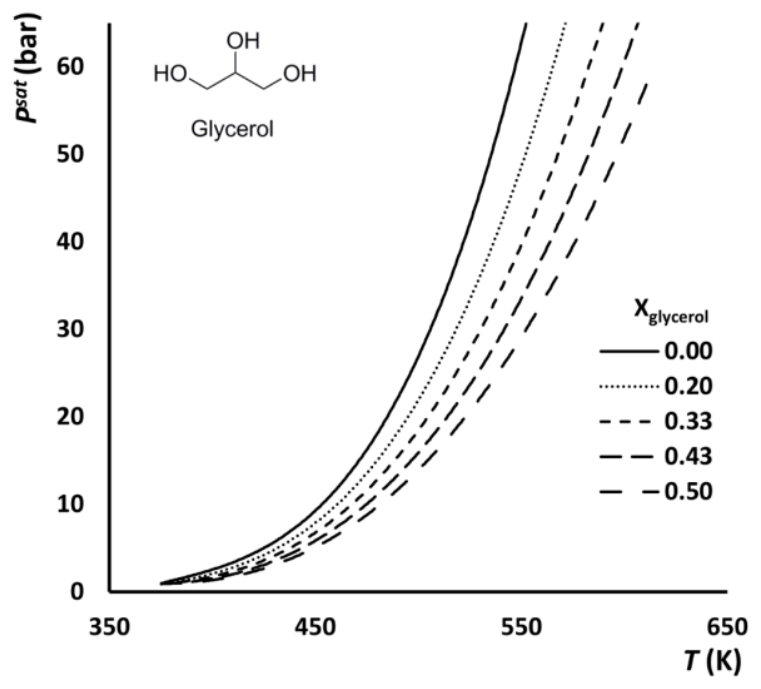

Figure A2.2.2. Saturated vapor pressure glycerol

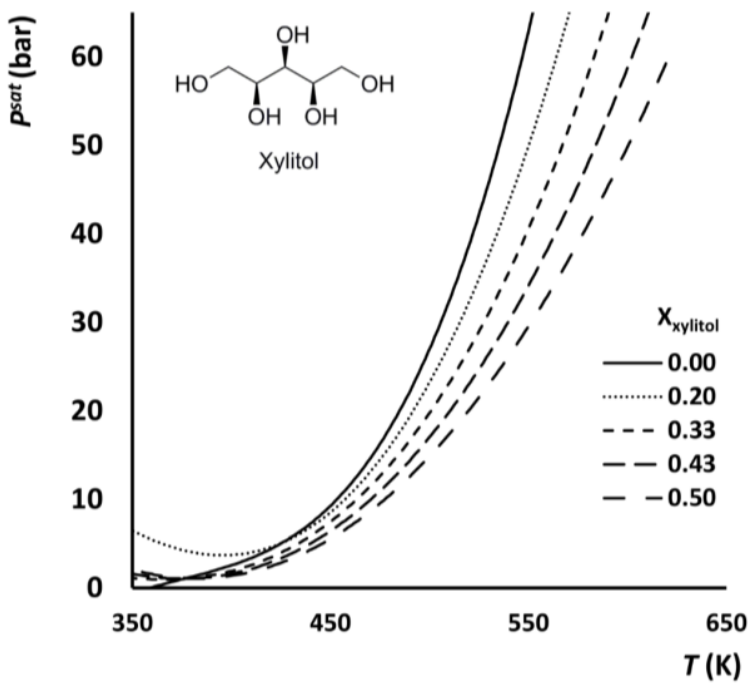

Figure A2.2.4. Saturated vapor pressure xylitol 


\section{A2.3. Equations}

\section{A2.3.1. Enthalpy}

$\Delta_{r} H^{\theta}=\sum_{\text {products }} v H_{f}^{\theta}-\sum_{\text {reactants }} v H_{f}^{\theta}$

A2.3.2. Enthalpy of reaction as a function of the temperature

$\Delta_{r} H(T)=\Delta_{r} H^{\theta}+\int_{T_{r e f}}^{T} \Delta_{r} c_{p}^{\theta} d T$

With the specific heat capacity, independent of temperature:

$\Delta_{r} c_{p}=\sum_{\text {products }} v C_{p, m}^{\theta}-\sum_{\text {reactants }} v c_{p, m}^{\theta}$

Specific heat capacity as a function of temperature

$c_{p}=A+B T+C T^{2}+D T^{-2}$

\section{A2.3.3. Enthalpy of reaction as a function of the temperature and the pressure}

Derived from ref $^{25}$

1. Total differential of the enthalpy

$$
\begin{aligned}
d H & =\left(\frac{\partial H}{\partial T}\right)_{P} d T+\left(\frac{\partial H}{\partial P}\right)_{T} d P \\
& =c_{p} d T+\left(\frac{\partial H}{\partial P}\right)_{T} d P
\end{aligned}
$$

$H=U+P V$

$\rightarrow d H=d U+V d P+P d V$

2. From the first law of thermodynamics

$$
\begin{aligned}
d U & =Q_{r e v}+W_{r e v} \\
& =Q_{r e v}-P d V
\end{aligned}
$$

with:

$Q_{r e v}=T d S$ 
results in:

$d U=T d S-P d V$

Inserting this in the total differential of the enthalpy (7) yields:

$$
\begin{aligned}
d H & =T d S-P d V+V d P+P d V \\
& =T d S+V d P
\end{aligned}
$$

3. From the differential of entropy for temperature and pressure:

$d S=\left(\frac{\partial S}{\partial T}\right)_{P} d T+\left(\frac{\partial S}{\partial P}\right)_{T} d P$

Substituting (12) into (11):

$$
\begin{aligned}
d H & =T\left[\left(\frac{\partial S}{\partial T}\right)_{P} d T+\left(\frac{\partial S}{\partial P}\right)_{T} d P\right]+V d P \\
& =T\left(\frac{\partial S}{\partial T}\right)_{P} d T+T\left(\frac{\partial S}{\partial P}\right)_{T} d P+V d P \\
& =T\left(\frac{\partial S}{\partial T}\right)_{P} d T+\left[T\left(\frac{\partial S}{\partial P}\right)_{T}+V\right] d P
\end{aligned}
$$

4. Equation (5) and (13)

$c_{p} d T+\left(\frac{\partial H}{\partial P}\right)_{T} d P=T\left(\frac{\partial S}{\partial T}\right)_{P} d T+\left[T\left(\frac{\partial S}{\partial P}\right)_{T}+V\right] d P$

So:

$c_{p}=T\left(\frac{\partial S}{\partial T}\right)_{P}$

And

$$
\left(\frac{\partial H}{\partial P}\right)_{T}=T\left(\frac{\partial S}{\partial P}\right)_{T}+V
$$

5. From the total differential for the Gibbs free energy:

$$
\begin{aligned}
& d G=V d P-S d T \\
& \left(\frac{\partial V}{\partial T}\right)_{P} d T=-\left(\frac{\partial S}{\partial P}\right)_{T}
\end{aligned}
$$


Substituting in (16):

$\left(\frac{\partial H}{\partial P}\right)_{T}=-T\left(\frac{\partial V}{\partial T}\right)_{P}+V$

6. Obtain final expression

$d H=c_{p} d T+\left[-T\left(\frac{\partial V}{\partial T}\right)_{P}+V\right] d P$

7. This can be simplified using the thermal expansion coefficient $\alpha$

$\alpha=\frac{1}{V}\left(\frac{\partial V}{\partial T}\right)_{P}$

$\rightarrow V \alpha=\left(\frac{\partial V}{\partial T}\right)_{P}$

Substituting:

$d H=c_{p} d T+\left[V-T\left(\frac{\partial V}{\partial T}\right)_{P}\right] d P$

$\rightarrow d H=c_{p} d T+[V-T V \alpha] d P$

$\rightarrow d H=c_{p} d T+[1-T \alpha] V d P$

\section{A2.3.4. Standard Gibbs free energy of reaction}

$\Delta_{r} G^{\theta}=\sum_{\text {product }} v G_{m}^{\theta}-\sum_{\text {reactants }} v G_{m}^{\theta}$

\section{A2.3.5. Gibbs free energy of reaction as function of temperature}

Gibbs-Helmholtz equation:

$\left(\frac{\partial}{\partial T} \frac{G}{T}\right)_{P}=-\frac{H}{T^{2}}$

Van 't Hoff equation:

$\ln K=-\frac{\Delta_{r} H}{R} \frac{1}{T}+\frac{\Delta_{r} S}{R}$

$\Delta_{r} G=-R T \ln K$ 


\section{A2.4. Data comparison}

The data from this work was compared with the data reported by Davda in 2003 to validate the proposed model. ${ }^{18}$ For this, the results were normalized to the number of $\mathrm{CO}$ molecules that are produced. The obtained trends are comparable for the Davda paper and our work. The deviation for ethylene glycol is $1 \mathrm{~kJ}^{\mathrm{mol}}{ }^{-1}$ which is considered to be acceptable for this type of study. The data for the water-gas-shift are in even better accordance with what has been published previously. The deviation for the liquid water-gas-shift in our model is caused by not taking into account the applied pressure to maintain in the liquid phase.

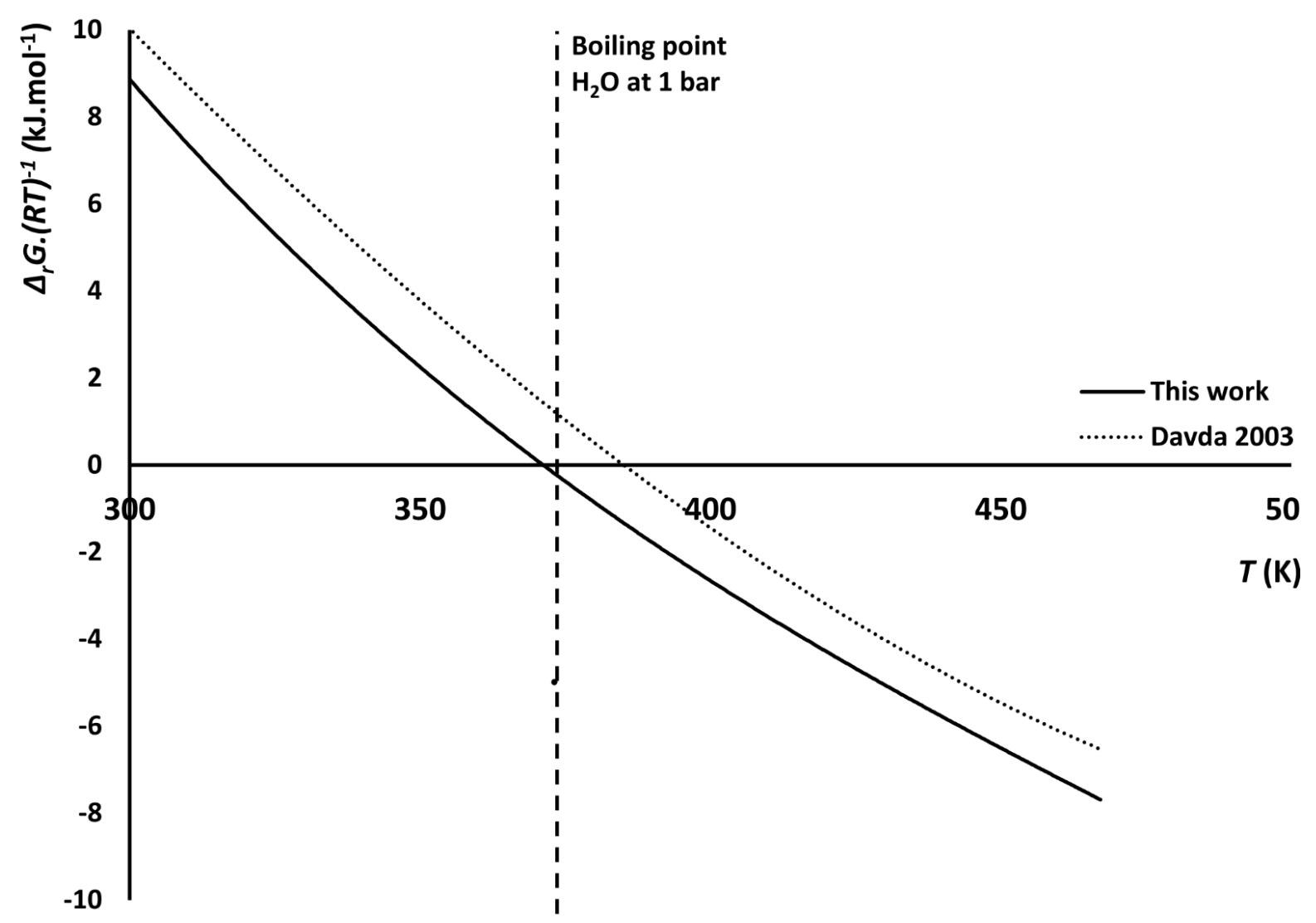

Figure A2.4.1 Gibbs free energy of APR of liquid ethylene glycol as a function of the temperature; our work and Davda 2003. The boiling point of water at 1 bar has been indicated by the vertical dotted line. 


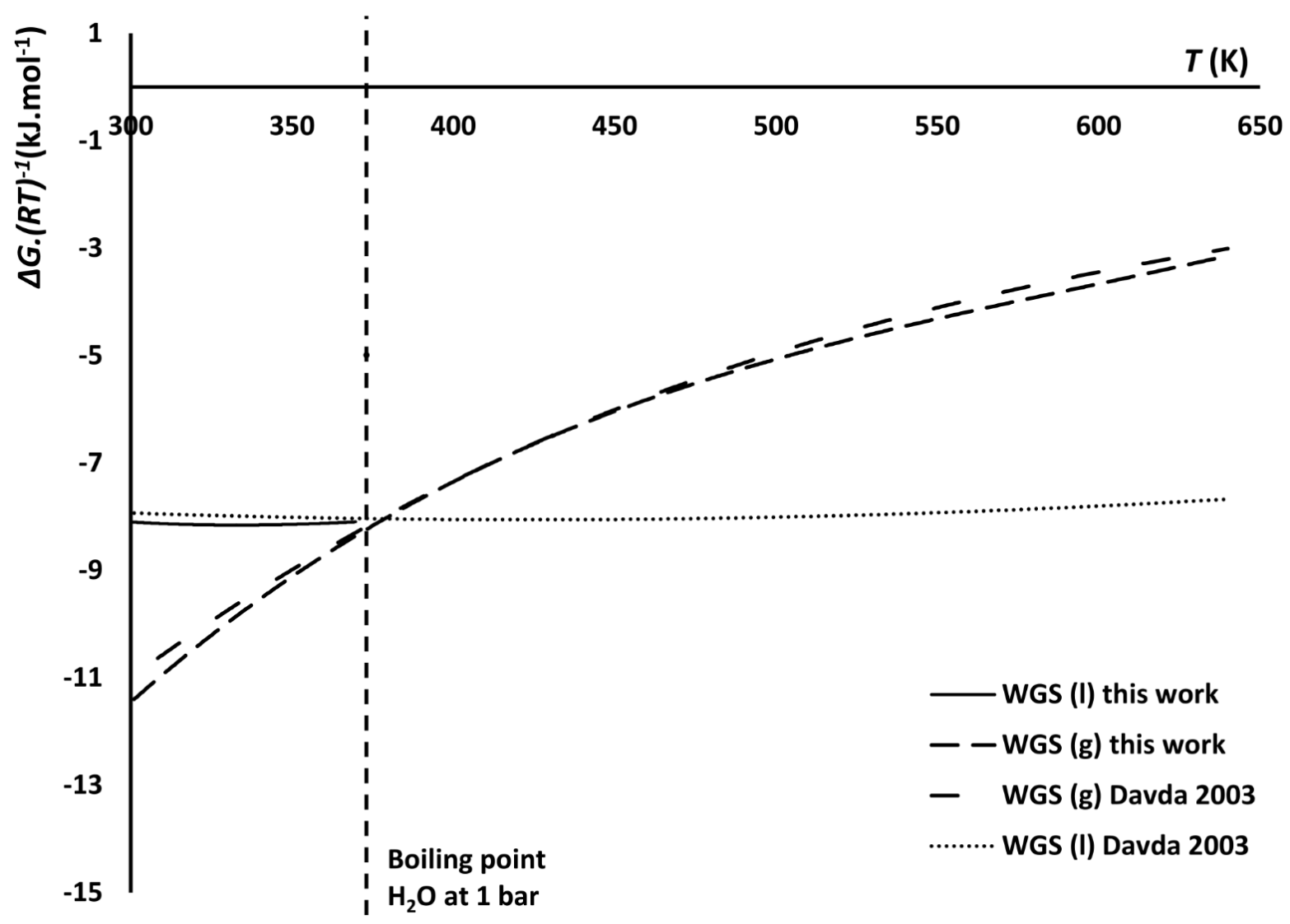

Figure A2.4.2 Gibbs free energy of the liquid and gas phase WGS as a function of the temperature; our work and Davda 2003. The boiling point of water at 1 bar has been indicated by the vertical dotted line. As the WGS in this work has been calculated at atmospheric pressure, the graph of the Gibbs free energy of reaction with water in the liquid phase is only shown for temperature below $373.15 \mathrm{~K}$. 


\title{
3 \\ High-pressure high-temperature microfluidics
}

\begin{abstract}
Aqueous-Phase Reforming (APR) is a promising hydrogen production method, where biomass is catalytically reformed under high-pressure and-high temperature reaction conditions. To eventually study APR, in this paper, we report a high-pressure and high-temperature microfluidic platform that can withstand temperatures up to $473 \mathrm{~K}$ and pressures up to 30 bar. As a first step, we studied the phase transition of four typical APR biomass model solutions, consisting of $10 \mathrm{wt} \%$ of ethylene glycol, glycerol, xylose or xylitol in MilliQ water. After calibration of the set-up using pure MilliQ water, a small increase in boiling point was observed for the ethylene glycol, xylitol and xylose solutions compared to pure water. Phase transition occurred through either explosive or nucleate boiling mechanisms, which was monitored in real-time in our microfluidic device. In case of nucleate boiling, the nucleation site could be controlled by exploiting the pressure drop along the microfluidic channel. Depending on the void fraction, various multiphase flow patterns were observed simultaneously. Altogether, this study will not only help to distinguish between bubbles resulting from a phase transition or APR product formation, but are also important from a heat and mass transport perspective.
\end{abstract}

Adapted from:

Ripken, R. M.; Schlautmann, S.; Sanders, R. G. P.; Gardeniers, J. G. E.; Le Gac, S., Monitoring phase transition of aqueous biomass model substrates by high-pressure and high-temperature microfluidics. ELECTROPHORESIS 2019, $40(4), 563-570$

Ripken, R. M.; Gardeniers, J. G. E.; Le Gac, S., Real-time monitoring of complex multiphase behavior in a high pressure and high temperature microfluidic chip. MicroTAS, Proceedings 2017 


\subsection{Introduction}

Hydrogen has been identified as a promising renewable energy vector to replace fossil fuels in the future, ${ }^{1-3}$ in particular when it is produced from biomass ${ }^{4}$ using Aqueous-Phase Reforming (APR). In APR, oxygenated carbohydrates that make up the biomass are reformed into hydrogen in aqueous solution. ${ }^{5}$ Typically, APR requires elevated temperatures and an external pressure, depending on the exact composition of the reaction mixture, to keep all reagents in the liquid phase. ${ }^{4}$

To determine the pressure that is required to maintain the APR reaction mixture in the liquid state, the phase transition of this solution must be known. For a single component the boiling point can easily be calculated as a function of the pressure using the Clapeyron equation. For multicomponent systems, however, the calculation is much more complex, as the components in the solution may interact with each other, which can lead to an increase or decrease in the boiling point. In Chapter 2, we have theoretically determined the boiling point of four APR model reaction mixtures, consisting of aqueous solutions of biomass model substrates (ethylene glycol, glycerol, xylose and xylitol), each as a function of the pressure for mole fractions up to 0.5 using the Redlich-Soave-Kwong Boston-Mathias equation of state ${ }^{6}$. We now aim to validate this theoretical model before studying APR experimentally.

To study the phase transition as well as to conduct chemical reactions such as biomass conversion, a microfluidic platform presents numerous advantages: as a result of their high surface-to-volume ratio, microfluidic devices provide more efficient heat and mass transfer compared to conventional reactors, excellent flow control, and require only small amounts of samples. ${ }^{7-11}$ Several microfluidic set-ups have been developed to cope with extreme reaction conditions. For example, Tiggelaar et al. ${ }^{12}$ proposed a glass-based microfluidic device with glued-in capillaries that could withstand pressures up to 690 bar. The Jensen group reported a system to perform catalytic reactions that can withstand both elevated temperatures and pressures, up to $673 \mathrm{~K}$ and $300 \mathrm{bar}$, respectively. ${ }^{13}$ In the latter device the pressurized part of the system was thermally insulated from the heated part to prevent the O-rings used in the connections from melting.

Before studying APR with a similar configuration, we first aim to evaluate the phase transition of model APR reaction mixtures. A number of microfluidic set-ups have specifically been described for studying phase transitions and for degassing processes. ${ }^{14-19}$ Some microfluidic devices contain 
a constriction in the microchannel, behind which vortices are created. Gas is trapped by these vortices, and bubbles are formed. ${ }^{14-15}$ Other systems make use of a local temperature shock ${ }^{16}$ or controlled decompression ${ }^{17}$ to induce nucleation or a stop-flow approach to ensure good control over the multiphase flow. ${ }^{18}$ In general, a microfluidic format has been proven to be up to 3 times as efficient compared to conventional set-ups in determining phase transition, ${ }^{14}$ notably by reducing the time for analysis from hours to minutes ${ }^{15}$ as thermodynamic equilibrium is reached quickly.

In this chapter, we present a versatile yet simple high-pressure and high-temperature (HPHT) microfluidic set-up, able to withstand temperatures up to $473 \mathrm{~K}$ and in which fluids (gas and/or liquid) can be pressurized up to 30 bar. As a first step, we experimentally determined the boiling points of typical APR reaction mixtures, consisting of $10 \mathrm{wt} \%$ aqueous biomass model solutions based on ethylene glycol, glycerol, xylose and xylitol. Knowing the phase transition of these solutions would allow distinguishing between gas product formation and a phase change when conducting APR in these devices. Furthermore, we studied the boiling mechanisms occurring at the microscale and examined how the gas-liquid flow pattern was evolving depending on the gas fraction in the microchannel. This study will be instrumental in predicting the multiphase flow pattern that is generated from hydrogen gas formation in APR, and how it influences the heat and mass transport in the channel.

\subsection{Materials and methods}

\subsubsection{Microfluidic device}

The core of our HPHT set-up consists of a glass/silicon microfluidic device (Figure 3.1). The transparency of the glass top layer allows monitoring the bubble nucleation and the gas/liquid flow regimes during the experiment, whereas silicon ensures good heat transfer to the fluidic channel. The design includes a 500- $\mu \mathrm{m}$ wide, $250-\mu \mathrm{m}$ deep and $20-\mathrm{cm}$ long meandering channel with a rectangular

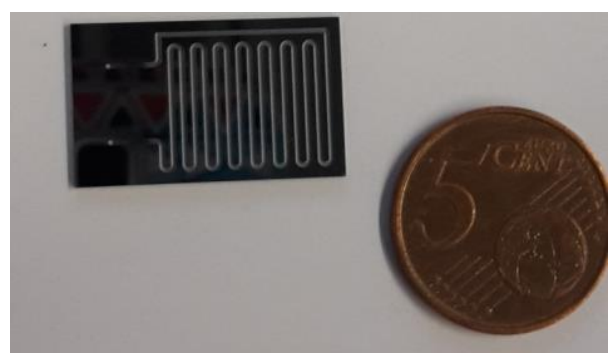

Figure 3.1. Glass/silicon microfluidic device containing a $0.2-\mathrm{m}$ long meandering channel, which is $500-\mu \mathrm{m}$ wide and $250-\mu \mathrm{m}$ deep. 
cross-section. The bonding area was optimized, with at least one time the channel width between the meanders, so that the device can withstand pressures up to 30 bar. The device was fabricated using standard microfabrication techniques. Briefly, after a photolithographic step, the channel was dry-etched $\left(-313 \mathrm{~K}, 10\right.$ mbar He pressure, $\mathrm{SF}_{6}$ flow rate $500 \mathrm{sscm}, \mathrm{C}_{4} \mathrm{~F}_{8}$ deposition $175 \mathrm{sscm}$, Adixen, AMS 100) into the silicon substrate (10-cm diameter, <100>, p/boron-doped, 525- $\mu \mathrm{m}$ thick, Okmetic). Using the same etching technique the inlet and outlet were machined from the backside of the silicon substrate. Subsequently, a 500-nm $\mathrm{SiO}_{2}$ layer was grown on the silicon substrate by wet oxidation at $1423 \mathrm{~K}$ for $26 \mathrm{~min}$ before it was anodically bonded to the glass substrate (MEMPax, 10-cm diameter, 500- $\mu \mathrm{m}$ thick, Schott AG).

\subsubsection{High-pressure and high-temperature set-up}

The entire HPHT set-up consists of two parts: a liquid handling system and a dedicated chip holder for the above described microfluidic device (Figure 3.2). The chip holder, which includes both the temperature control and the fluidic connections, was CNC-machined from PEEK, a durable and chemically inert material with good thermal insulation properties, which therefore minimizes heat loss to the surroundings. The material properties of PEEK determine the maximum working temperature of the set-up, which is $523 \mathrm{~K}$ in this case. The bottom plate of the chip holder contained an opening, acting as an optical window for real-time monitoring of the multiphase flow pattern. A ceramic heater (HT24S 24W, Thorlabs) was placed underneath the microdevice at the silicon side and connected to a temperature feedback loop. The temperature was regulated and monitored by two K-type thermocouples (CHAL-002, OMEGA) placed between the heater and the silicon side of the device: one for the feedback to the heater and one for the temperature read-out. The thermocouples were first calibrated and the homogeneity of the temperature field in the microfluidic device was investigated using a thermal camera (FLIR ONE Gen 3 Pro - IOS, resolution $0.1 \mathrm{~K})$.

The pressure was regulated by back pressure regulators (BPR) of 75 (5.2 bar) or 100 psi (6.9 bar) maximum back pressure (IDEX Health \& Science) that were connected at the outlet of the chip holder. A pressure meter was included between the outlet of the microfluidic device and the BPR to monitor pressure fluctuations during the experiments. 
The fluidic connections between the inlet and outlet of the device and the top plate of the chip holder consisted of silicone O-rings (ID $1.07 \mathrm{~mm}$; OD $1.27 \mathrm{~mm}$, ERIKS) and PEEK ferrules (F126H, Upchurch). PEEK tubing (ID $125 \mu \mathrm{m}$; OD 1/32 inch, Inacom) was used to transport the fluids in and out of the system. The liquid was infused by a syringe pump (Chemyx Inc. Fusion 100 ) and a gastight $250-\mu \mathrm{L}$ glass syringe (Hamilton).

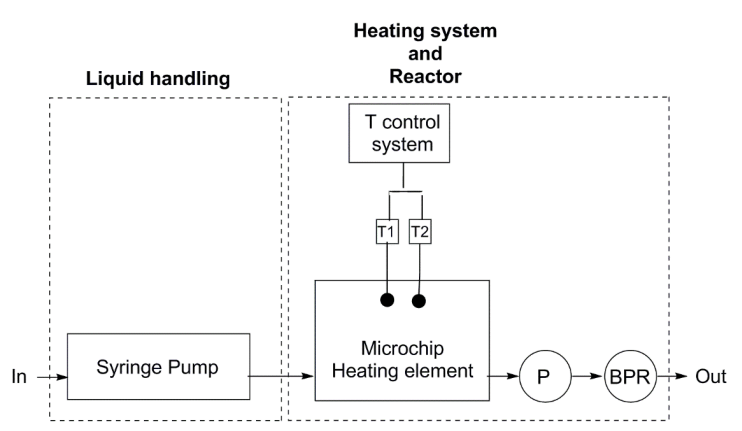

A.

Figure 3.2. A. Schematic representation of the complete set-up consisting of a liquid handling part and a chip holder, integrating the microfluidic device and the heater. $\mathrm{P}=$ pressure meter; $\mathrm{BPR}=\mathrm{Back}$ Pressure Regulator. $\mathrm{B}$. PEEK chip holder with 1. Bottom plate with optical window, 2. Microfluidic device, 3. Ceramic heater, 4. Top plate with fluidic connections.

\subsubsection{Phase transition studies and multiphase flow regimes}

Four solutions of $10 \mathrm{wt} \%$ biomass in MilliQ water were prepared, $10 \mathrm{wt} \%$ being the concentration typically used in APR. ${ }^{4,20}$ Ethylene glycol, glycerol, D-xylose and xylitol (Sigma-Aldrich) were used as received. MilliQ water without any substrate acted as a reference to calibrate the system. The solutions were introduced into the system at a flow rate of $10 \mu \mathrm{L} \cdot \mathrm{min}^{-1}$. For each measurement the pressure was fixed by the BPR, while the temperature was increased by $5 \mathrm{~K} \cdot \mathrm{min}^{-1}$ starting from room temperature until boiling was observed. The BPR was omitted in the experiments in which the boiling point at atmospheric pressure was determined. The temperature and pressure were monitored during the complete duration of the experiment. A small video camera (DNT, DigiMicro Mobile, maximum 30 frames. $\mathrm{s}^{-1}$ ) was used to determine when boiling occurred and to visualize the resulting multiphase flow in the microchannel. Data for each biomass solution were recorded in triplicates. 


\subsection{Results and discussion}

A straightforward, relatively simple system was developed for high-temperature high-pressure experiments up to $523 \mathrm{~K}$ and at least 30 bar. Our plug-and-play set-up allows quickly changing microfluidic devices, so that the microreactor can be easily adapted to the requirements of the experiment. The microfluidic device in this work can not only be used to determine thermodynamic properties, and to determine the phase transition of mixtures, but also for conducting chemical reactions, such as APR, at real operation conditions, as used in industrially relevant processes. While our set-up based on PEEK is suitable to conduct experiments at temperatures up to $523 \mathrm{~K}$, the same chip holder design could be realized in, for instance stainless steel, for experiments at even higher temperatures.

\subsubsection{Temperature calibration}

According to previous work ${ }^{21-25}$, the temperature measured outside the microfluidic device can significantly differ from the temperature inside the fluidic channels, which is typically caused by a difference in thermal conductivity between the bulk material and the fluid ${ }^{25}$. Therefore, the HPHT set-up was first calibrated with pure MilliQ water. At a flow rate of $10 \mu \mathrm{L} \cdot \mathrm{min}^{-1}$ the thermal entrance length was found to be negligible $(<1 \mathrm{~mm})$, so that the fluid reached almost instantaneously the temperature of the heater. Comparison of the experimental boiling points (Figure 3.3, dashed line) with theoretical values (Figure 3.3, bold line) revealed a systematic offset

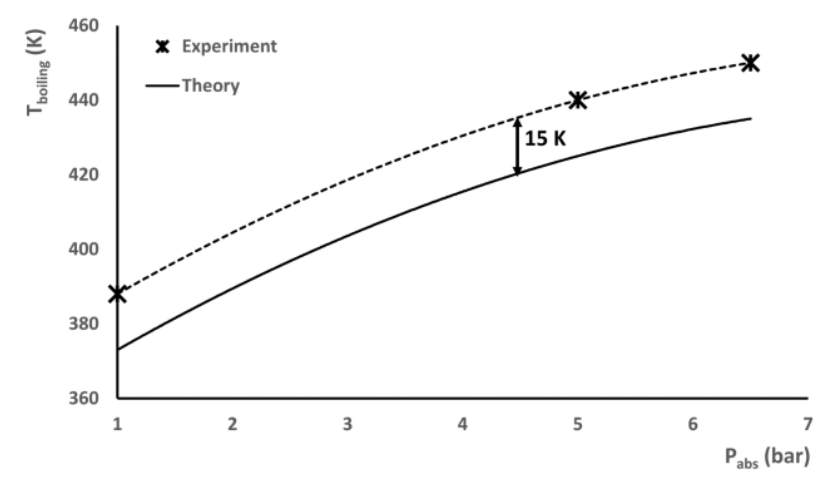

Figure 3.3.Calibration of the HPHT set-up with pure MilliQ water. The dashed line corresponds to the experimental results from this work, while the solid line represents theoretical data.

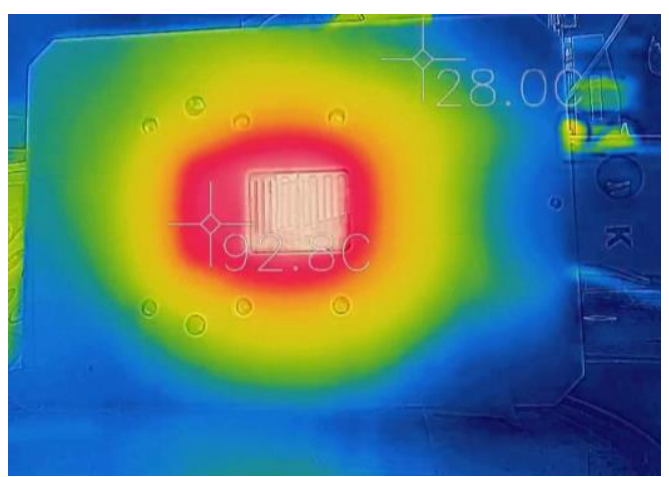

Figure 3.4. Temperature distribution in the setup (bottom plate of the chip holder with microfluidic device) visualized using a thermal camera. Here, temperatures are ranging from 301 (green) to $373 \mathrm{~K}$ (white). 
of $15 \mathrm{~K}$, which is in the same range as previously reported by Dodge et al. for a similar set-up comprising an ITO heater ${ }^{24}$. All experiments described in this paper have been corrected for this offset. Next, we evaluated the temperature distribution in the device using a thermal camera: a homogeneous temperature field was found (Figure 3.4), and within a matter of seconds the desired temperature was reached in the entire microfluidic device.

\subsubsection{Experimental boiling points and comparison with theoretical data}

After calibration of the HPHT set-up, the liquid to gas phase transition for the four $10 \mathrm{wt} \%$ model substrate/water solutions was studied. The results are presented in Figure 3.5, where the dashed orange bar represents the theoretical boiling point of pure MilliQ water. For each substrate solution, the phase transition temperature was measured in triplicate. Addition of $10 \mathrm{wt} \%$ of ethylene glycol, xylose and xylitol to water systematically increased the boiling point by $5 \mathrm{KC}$ compared to pure MilliQ water, whereas our theoretical model predicted an increase of only 1-2 K. ${ }^{6}$ Surprisingly, the addition of glycerol did result in an even higher boiling point than expected with less reproducibility than for the other three substrates.

This small yet unexpected difference of $5 \mathrm{~K}$ is unlikely to be caused by a pressure drop in the microchannel, which can be calculated by the Darcy-Weisbach equation:

$$
\frac{\Delta P}{L}=\frac{128}{\pi} \frac{\mu}{D^{4}} \Phi_{V}
$$

With $P$ the pressure, $L$ the length of the channel, $D$ its hydraulic diameter, $\mu$ the dynamic viscosity and $\Phi_{V}$ the volumetric flow rate. When using MilliQ at room temperature the pressure drop over our $20 \mathrm{~cm}$ channel is $38.6 \mathrm{~Pa}$, which is negligible. As the viscosity decreases when increasing the temperature, the pressure drop is even lower at higher temperatures.

Another possible explanation could be the differences in the physical properties between the biomass solutions and pure water, since these properties affect the heat transfer to the fluid. The heat transferred is described by:

$$
Q=h A\left(T_{\text {wall }}-T_{\text {bulk }}\right)
$$




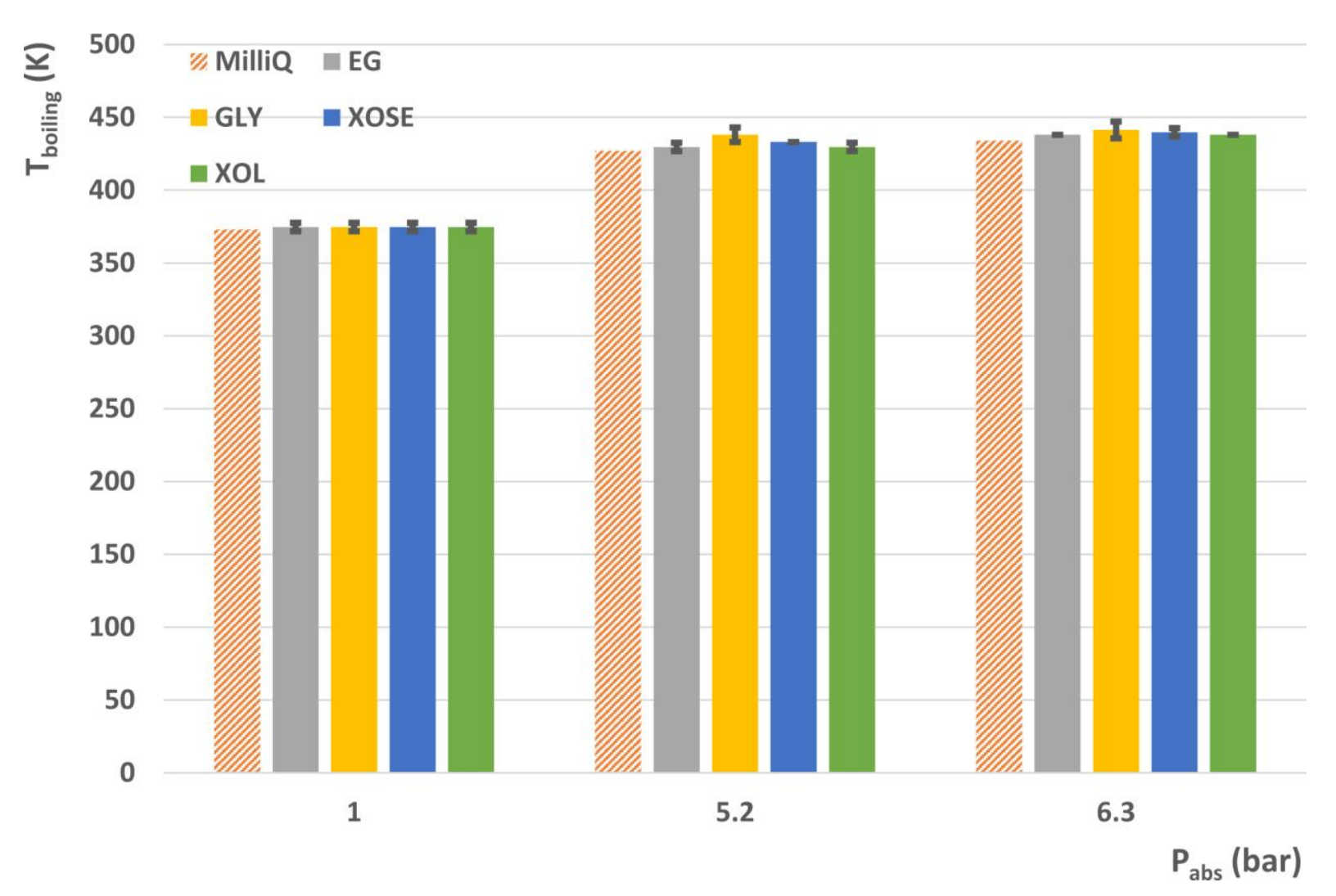

Figure 3.5. Experimental boiling points of $10 \mathrm{wt} \%$ ethylene glycol (EG, grey), glycerol (GLY, yellow), xylose (XOSE, blue) and xylitol (XOL, green) in MilliQ water as a function of absolute pressure. The theoretical boiling point of pure MilliQ water is indicated in the dashed orange bar. The boiling point of each substrate was measured in triplicate.

With $Q$ being the total heat transferred and $A$ the area through which heat is transported. For a system operating under laminar flow conditions, the heat transfer coefficient $h$ at a given location $x$ in the channel can be expressed as:

$$
h=\frac{N u_{x} k}{x}=0.332 \operatorname{Re}_{x}^{1 / 2} \operatorname{Pr}^{1 / 3}
$$

With $N u_{x}$ the dimensionless Nusselt number at location $x$ along the channel, $k$ the thermal conductivity of the fluid and $\operatorname{Re}$ and $\operatorname{Pr}$ the Reynolds and Prandtl numbers, respectively. As our APR model solutions contain very low substrate concentrations, $R e$ is not expected to change significantly compared to pure water. $P r$, however, depends not only on the viscosity $\mu$ and the specific heat capacity $c_{p}$, but also on the thermal conductivity $k$ according to:

$$
\operatorname{Pr}=\frac{\mu c_{p}}{k}
$$


Consequently, $\operatorname{Pr}$ varies more significantly with the solution composition. Therefore, the heat transfer coefficient of the biomass solutions is mainly changing as a result of the change in thermal conductivity of the solution. For example, a $10 \mathrm{wt} \%$ solution of ethylene glycol in water has a thermal conductivity of $393 \mathrm{~mW} \cdot \mathrm{m}^{-1} \cdot \mathrm{K}^{-1}$ at 1 bar and $372.8 \mathrm{~K},{ }^{26}$ compared to $677 \mathrm{~mW} \cdot \mathrm{m}^{-1} \cdot \mathrm{K}^{-1}$ for pure water under the same conditions. ${ }^{27}$ As a result, the heat transfer coefficient of the model solutions is lower than that of pure water, which explains the slightly higher offset value we observed for the biomass solutions, which is in the order of the observed $5 \mathrm{~K}$. However, this difference in heat transfer can still not account for the higher boiling point of glycerol, as the thermal conductivity of pure glycerol is comparable to that of pure ethylene glycol $\left(285 \mathrm{~mW} . \mathrm{m}^{-}\right.$ ${ }^{1} \cdot \mathrm{K}^{-1}$ for ethylene glycol ${ }^{26}$ against $258 \mathrm{~mW} \cdot \mathrm{m}^{-1} \cdot \mathrm{K}^{-1}$ for glycerol. ${ }^{28}$ More research is thus required to explain these differences. Taking into account the additional offset as a result of the difference in thermal conductivity, and correcting the experimental boiling points accordingly, it can be concluded that for APR experiments with ethylene glycol, xylose and xylitol at a concentration of $10 \mathrm{wt} \%$ the required pressure to keep the system in the liquid phase can be approximated by that required for pure water.

It should be noted that a $10 \mathrm{wt} \%$ substrate solution, depending on the molecular weight of the substrate, corresponds to only a very small mole fraction between 0.01 and 0.03 , which is caused by the large difference between the molecular weight of water and the substrate molecule. Based on our previous work, ${ }^{6}$ such a small mole fraction of biomass would not significantly affect the boiling point compared to pure water, which is in agreement with the experimental results presented here for ethylene glycol, xylose and xylitol.

To further validate the theoretical model a wider range of substrate mole fractions or higher weight percentages should be considered, as a higher biomass content would increase the boiling temperature, according to our theoretical model ${ }^{6}$. Specifically, a biomass mole fraction of 0.5 would increase the boiling point by 8 to $24 \mathrm{~K}$, depending on the substrate, which corresponds to solutions with weight percentages up to $90 \%$. Using such high concentrations of biomass would, however, not only change the boiling point, but also other physical properties of the solution, such as the aforementioned thermal conductivity and viscosity, which would significantly affect the transport phenomena in our microfluidic system. At the same time, such high biomass concentrations are less relevant to study, since APR solutions typically contain $10 \mathrm{wt} \%$ of the 
substrate. At higher weight percentages, carbon deposition becomes significant in APR, as well as other side reactions such as methanation, which are detrimental for the hydrogen yield ${ }^{20}$.

\subsubsection{On-chip boiling mechanisms: explosive and nucleate boiling}

When studying the phase transition of these biomass model solutions, two boiling mechanisms were observed in the microfluidic device: eruption or explosive boiling (Figure 3.6A) and nucleate boiling (Figure 3.6B). Explosive boiling was most often observed in our experiments, in good agreement with Zhang et al., who reported that this boiling mechanism is predominant in microchannels, ${ }^{29}$ in particular for systems with a small Péclet number. ${ }^{30}$

Typically, in the case of explosive boiling, the liquid was pushed away from the nucleation site both in and against the liquid flow direction. After boiling was achieved, the flow direction was reversed and the channel was partly cleared from any liquid. We also witnessed a fluctuating gas/liquid front, as already described in the literature. ${ }^{30}$

The resulting gas/liquid multiphase system was highly uncontrollable in terms of flow rate and pressure. Every bubble nucleation caused a local pressure increase of several bar, as a result of the Laplace pressure that has to be overcome before a bubble could be formed. The pressure in the system, which initially increased just before bubble nucleation, subsequently dropped by up to 4 bar and kept fluctuating during boiling. Furthermore, when the gas fraction in the fluidic channel increased, the liquid plug velocity accelerated to fulfill the law of mass conservation. Altogether, the flow rate and pressure continuously fluctuated when saturation was reached.

The second boiling mechanism, nucleate boiling, is typically promoted by the presence of contamination on the microchannel wall or solid particles in the bulk of the liquid. Defects on the microchannel walls also promote this boiling mechanism, which explains the observed nucleate boiling in our set-up. The method of fabrication of our microchannels, deep reactive ion etching, is a cyclic process that forms small "scallops" at the inner walls of the etched structures, which can act as nucleation sites. In theory, after nucleation, the bubble grows until the buoyancy forces become greater than the interfacial tension forces at the microchannel wall. ${ }^{31}$ In our experiments, however, the bubble seemed to grow until it reached a size equal to that of the microchannel width, i.e., $500 \mu \mathrm{m}$. The bubble subsequently detached when the liquid pressure behind the bubble was 
A.

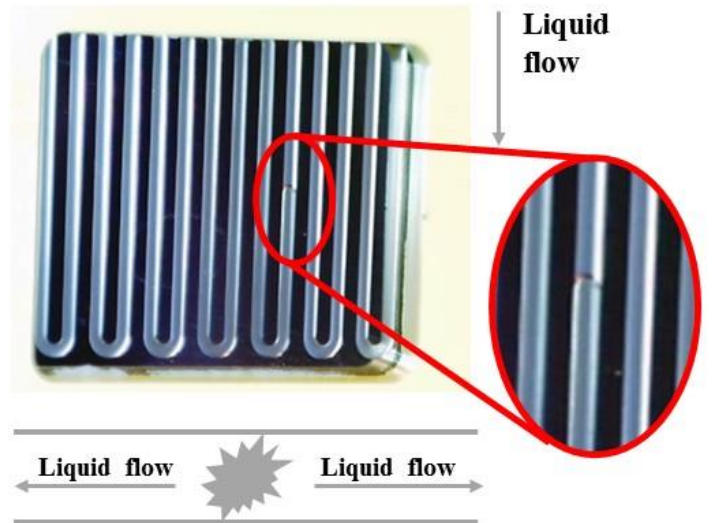

B.

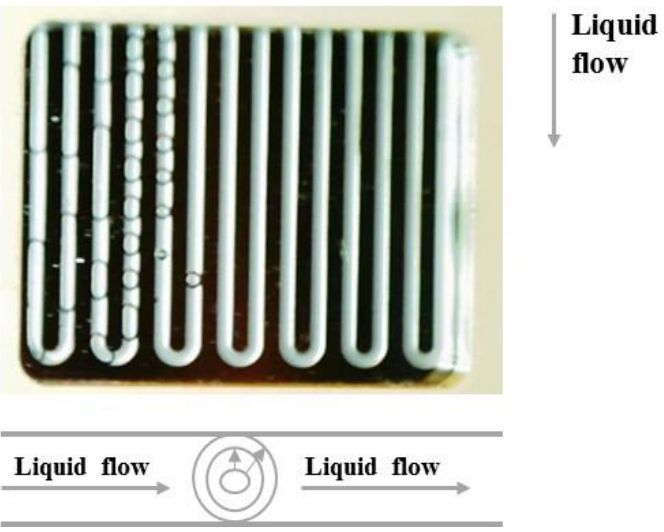

Figure 3.6. Observed boiling mechanisms. In eruption or explosive boiling (A) the channel is completely filled with gas behind the nucleation site. In nucleate boiling (B) gas bubbles are formed.

high enough to expel it from its nucleation site. Only when the temperature in the microchannel was far above the boiling temperature, the bubble was released at a smaller size. In the case of nucleate boiling, the pressure in the system increased by up to 2 bar before it dropped below the pressure set by the back pressure regulator after the first bubble was formed. In contrast to eruption boiling, the gas/liquid multiphase system was stable and yielded an alternating gas-liquid flow pattern, as discussed in the next section.

\subsubsection{Controlling the bubble nucleation site and multiphase flow patterns}

When nucleate boiling occurred, the location in the microchannel where the bubble formed could be controlled by taking advantage of the pressure drop along the channel, resulting from the flowing liquid. Concretely, when the temperature was slowly increased, the nucleation site shifted further upstream towards the inlet of the microchannel, where the pressure was higher. In other words, for a bubble to nucleate closer to the inlet, a temperature increase of only tenths of degrees was required to overcome the higher pressure closer to the inlet (Figure 3.7).

The transition from a homogeneous liquid phase to a gas-liquid system, as takes place during boiling, can be translated to the APR process where gaseous products form in a liquid phase. The formation of bubbles and multiphase flow patterns highly influence heat and mass transport and, in turn, the efficiency of the reaction. Therefore, we also studied the evolution of the two-phase flow and the transition between the different multiphase flow regimes. The resulting gas/liquid 
flow after saturation was found to be strongly dependent on the gas fraction. In this work, we define the gas or void fraction as the chordal void fraction, which is the ratio between the lengths of the gas plugs divided by the total considered flow length:

$$
\alpha_{\text {chordal }}=\frac{\text { length }_{\text {gas }}}{\text { length }_{\text {gas plugs }}+\text { length }_{\text {liquid } \text { plugs }}}
$$

Three different flow regimes were observed depending on the void fraction: (i) bubbly flow, (ii) Taylor flow and (iii) a high void fraction flow regime, which could correspond to an annular, a mist and/or a dry-out regime (Figure 3.7). The flow pattern is considered to be bubbly until the size of the gas bubble equals the hydraulic diameter of the microchannel. A Taylor flow is characterized by gas plugs, whose length is greater than the channel width.

The void fraction directly related to the observed flow regimes, as well as the transition from one flow pattern to another one (Figure 3.8). Multiple flow regimes were observed simultaneously in a single device over the entire void fraction range. The transition from a bubbly flow to a Taylor flow occurred at a void fraction of circa 0.05 , whereas the transition from a Taylor regime to the high void fraction flow took place at a void fraction of 0.8 , which is in accordance with the literature ${ }^{17}$. In the Taylor flow regime, the gas plugs expand as a result of the pressure decrease along the channel. Furthermore, boiling also occurred further downstream from the nucleation site, creating additional gas, which is taken up by the already existing gas plugs. At the same time, the velocity of the fluid plugs increases downstream to fulfill the conservation of mass. In non-boiling gas-liquid systems in a bubbly or Taylor flow regime, a thin liquid film of $1-10-\mu \mathrm{m}$ thick ${ }^{17}$ exists around the gas phase in the case of hydrophilic microchannel walls. However, in boiling systems it is highly likely that film boiling occurs in this layer, breaking up that thin liquid film. ${ }^{32}$

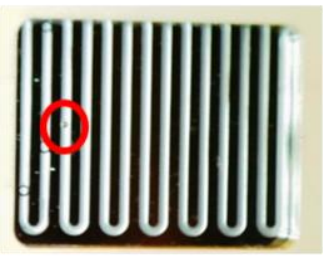

6 bar; $433 \mathrm{~K}$

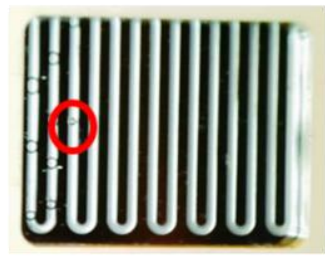

6 bar; $433 \mathrm{~K}$

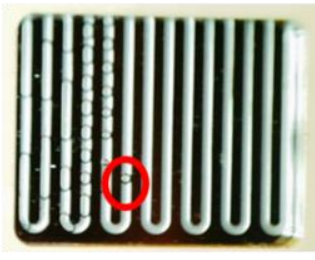

6 bar; $434 \mathrm{~K}$

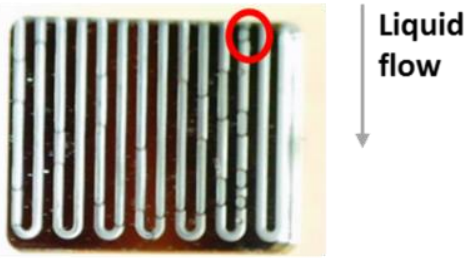

6 bar; $435 \mathrm{~K}$

Figure 3.7 Nucleation site shifting towards the inlet when the temperature is increased from $433 \mathrm{~K}$ to $435 \mathrm{~K}$ under a constant pressure of 6 bar. 


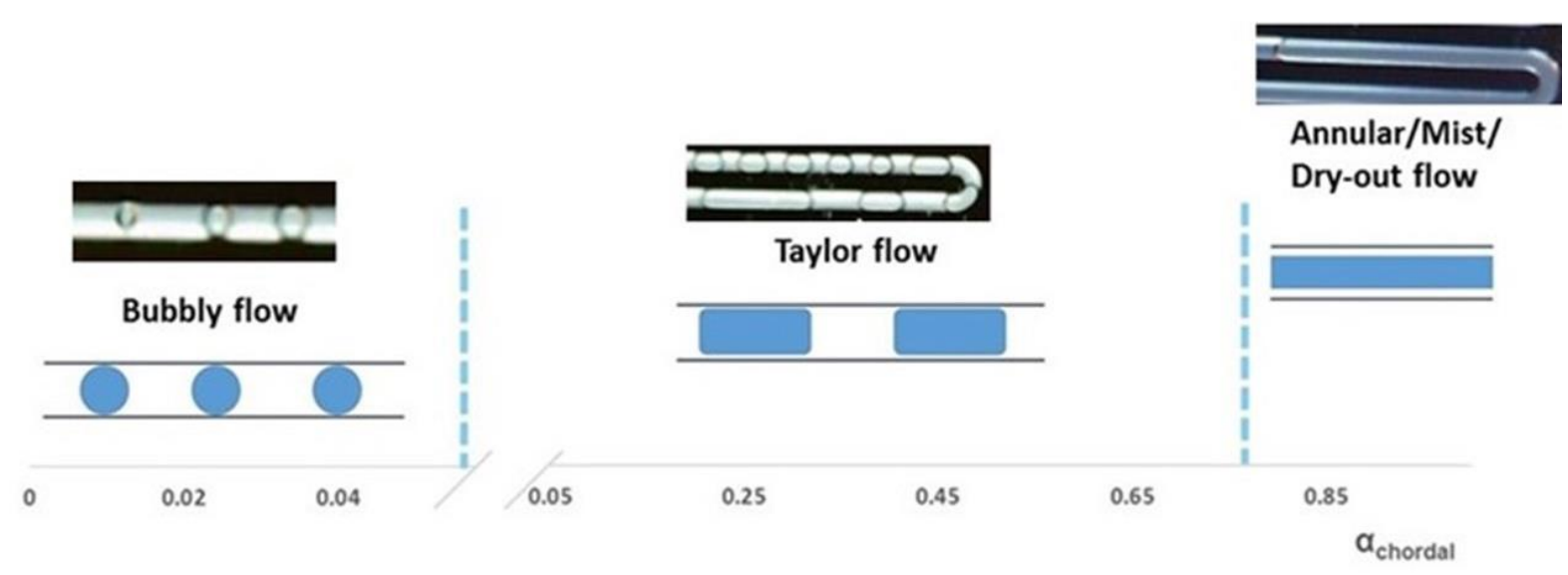

Figure 3.8. Observed flow regimes as a function of the chordal void fraction. From left to right bubbly flow, Taylor flow and high void fraction flow regime, which may consist of an annular, a mist and/or a dry-out flow.

\subsection{Conclusion}

In this work, we report a versatile high-pressure and high-temperature microfluidic set-up suitable towards studying APR, which can be operated at temperatures up to $473 \mathrm{~K}$ and pressures up to 30 bar. As a first step, we evaluated the phase transition of typical $10 \mathrm{wt} \%$ APR biomass model solutions of ethylene glycol, glycerol, xylose and xylitol in MilliQ. after calibrating the system with pure MilliQ water. The addition of three of these substrates in small amounts did not significantly affect the boiling point of pure water. Knowing the phase behavior of the model substrate solutions will allow us in the future to distinguish between a phase transition and the formation of gaseous products during APR. We also characterized the flow regimes resulting from gas generation by either boiling or reaction products, by their chordal void fraction. Several flow patterns, from a bubbly flow to combined high void fraction flow regimes were observed simultaneously in a single microfluidic device.

\section{References}

1. Balat, H.; Kirtay, E., Hydrogen from biomass - Present scenario and future prospects. International Journal of Hydrogen Energy 2010, 35 (14), 7416-7426.

2. Ball, M.; Weeda, M., The hydrogen economy - Vision or reality? International Journal of Hydrogen Energy 2015, 40 (25), 7903-7919. 
3. Hanley, E. S.; Deane, J. P.; Gallachoir, B. P. O., The role of hydrogen in low carbon energy futures-A review of existing perspectives. Renewable \& Sustainable Energy Reviews 2018, 82, 3027-3045.

4. Chheda, J. N.; Huber, G. W.; Dumesic, J. A., Liquid-phase catalytic processing of biomassderived oxygenated hydrocarbons to fuels and chemicals. Angewandte Chemie-International Edition 2007, 46 (38), 7164-7183.

5. Cortright, R. D.; Davda, R. R.; Dumesic, J. A., Hydrogen from catalytic reforming of biomass-derived hydrocarbons in liquid water. Nature 2002, 418 (6901), 964-967.

6. Ripken, R. M.; Meuldijk, J.; Gardeniers, J. G. E.; Le Gac, S., Influence of the Water Phase State on the Thermodynamics of Aqueous-Phase Reforming for Hydrogen Production. Chemsuschem 2017, 10 (24), 4909-4913.

7. Song, Y. J.; Hormes, J.; Kumar, C., Microfluidic synthesis of nanomaterials. Small 2008, 4 (6), 698-711.

8. Sackmann, E. K.; Fulton, A. L.; Beebe, D. J., The present and future role of microfluidics in biomedical research. Nature 2014, 507 (7491), 181-189.

9. Theberge, A. B.; Courtois, F.; Schaerli, Y.; Fischlechner, M.; Abell, C.; Hollfelder, F.; Huck, W. T. S., Microdroplets in Microfluidics: An Evolving Platform for Discoveries in Chemistry and Biology. Angewandte Chemie-International Edition 2010, 49 (34), 5846-5868.

10. Mark, D.; Haeberle, S.; Roth, G.; von Stetten, F.; Zengerle, R., Microfluidic lab-on-a-chip platforms: requirements, characteristics and applications. Chemical Society Reviews 2010, 39 (3), 1153-1182.

11. Cheng, X.; Ooms, M. D.; Sinton, D., Biomass-to-biocrude on a chip via hydrothermal liquefaction of algae. Lab on a Chip 2016, 16 (2), 256-260.

12. Tiggelaar, R. M.; Benito-Lopez, F.; Hermes, D. C.; Rathgen, H.; Egberink, R. J. M.; Mugele, F. G.; Reinhoudt, D. N.; van den Berg, A.; Verboom, W.; Gardeniers, H., Fabrication, mechanical testing and application of high-pressure glass microreactor chips. Chemical Engineering Journal 2007, 131 (1-3), 163-170.

13. Keybl, J.; Jensen, K. F., Microreactor System for High-Pressure Continuous Flow Homogeneous Catalysis Measurements. Industrial \& Engineering Chemistry Research 2011, 50 (19), 11013-11022. 
14. Song, W.; Fadaei, H.; Sinton, D., Determination of Dew Point Conditions for CO2 with Impurities Using Microfluidics. Environmental Science \& Technology 2014, 48 (6), 3567-3574.

15. Mostowfi, F.; Molla, S.; Tabeling, P., Determining phase diagrams of gas-liquid systems using a microfluidic PVT. Lab on a Chip 2012, 12 (21), 4381-4387.

16. Sullivan, M. T.; Angelescu, D. E., Microfluidic Bubble Point Measurement Using Thermal Nucleation. Energy \& Fuels 2016, 30 (4), 2655-2661.

17. Fisher, R.; Shah, M. K.; Eskin, D.; Schmidt, K.; Singh, A.; Molla, S.; Mostowfi, F., Equilibrium gas-oil ratio measurements using a microfluidic technique. Lab on a Chip 2013, 13 (13), 2623-2633.

18. Pinho, B.; Girardon, S.; Bazer-Bachi, F.; Bergeot, G.; Marre, S.; Aymonier, C., A microfluidic approach for investigating multicomponent system thermodynamics at high pressures and temperatures. Lab on a Chip 2014, 14 (19), 3843-3849.

19. Bao, B.; Riordon, J.; Mostowfi, F.; Sinton, D., Microfluidic and nanofluidic phase behaviour characterization for industrial CO2, oil and gas. Lab on a Chip 2017, 17 (16), 27402759.

20. Davda, R. R.; Shabaker, J. W.; Huber, G. W.; Cortright, R. D.; Dumesic, J. A., A review of catalytic issues and process conditions for renewable hydrogen and alkanes by aqueous-phase reforming of oxygenated hydrocarbons over supported metal catalysts. Applied Catalysis BEnvironmental 2005, 56 (1-2), 171-186.

21. Graham, E. M.; Iwai, K.; Uchiyama, S.; de Silva, A. P.; Magennis, S. W.; Jones, A. C., Quantitative mapping of aqueous microfluidic temperature with sub-degree resolution using fluorescence lifetime imaging microscopy. Lab on a Chip 2010, 10 (10), 1267-1273.

22. Sadler, D. J.; Changrani, R.; Roberts, P.; Chou, C. F.; Zenhausern, F., Thermal management of BioMEMS: Temperature control for ceramic-based PCR and DNA detection devices. Ieee Transactions on Components and Packaging Technologies 2003, 26 (2), 309-316.

23. Guijt, R. M.; Dodge, A.; van Dedem, G. W. K.; de Rooij, N. F.; Verpoorte, E., Chemical and physical processes for integrated temperature control in microfluidic devices. Lab on a Chip 2003, $3(1), 1-4$.

24. Dodge, A.; Turcatti, G.; Lawrence, I.; de Rooij, N. F.; Verpoorte, E., A microfluidic platform using molecular beacon-based temperature calibration for thermal dehybridization of surface-bound DNA. Analytical Chemistry 2004, 76 (6), 1778-1787. 
25. Iles, A.; Fortt, R.; de Mello, A. J., Thermal optimisation of the Reimer-Tiemann reaction using thermochromic liquid crystals on a microfluidic reactor. Lab on a Chip 2005, 5 (5), 540-544. 26. MEGlobal, Ethylene Glycol: Product Guide. 2008.

27. Toolbox, E. Water - Thermal Conductivity. https://www.engineeringtoolbox.com/waterliquid-gas-thermal-conductivity-temperature-pressure-d_2012.html.

28. Toolbox, E., Thermal Conductivities for some common Liquids. 2008.

29. Zhang, L. A.; Wang, E. N.; Goodson, K. E.; Kenny, T. W., Phase change phenomena in silicon microchannels. International Journal of Heat and Mass Transfer 2005, 48 (8), 1572-1582. 30. Peles, Y. P.; Yarin, L. P.; Hetsroni, G., Evaporating two-phase flow mechanism in microchannels. In Design, Test, and Microfabrication of Mems and Moems, Pts 1 and 2, Courtois, B.; Crary, S. B.; Ehrfeld, W.; Fujita, H.; Karam, J. M.; Markus, K., Eds. 1999; Vol. 3680, pp 226-236. 31. Xu, J. J.; Chen, B. D.; Xie, T. Z. Z., Experimental and theoretical analysis of bubble departure behavior in narrow rectangular channel. Progress in Nuclear Energy 2014, 77, 1-10.

32. Gambaryan-Roisman, T., Liquids on porous layers: wetting, imbibition and transport processes. Current Opinion in Colloid \& Interface Science 2014, 19 (4), 320-335. 


\title{
4
}

\section{Aqueous-Phase Reforming in a microreactor: the role of surface bubbles}

\begin{abstract}
In heterogeneous catalysis, the creation of gaseous products as bubbles in a liquid phase on the catalytic surface is associated with slip phenomena. In a microreactor, the slip length at the gas/liquid interface is in the same order of magnitude as the reactor dimensions, which can affect fluid dynamics and transport phenomena. Here, we studied the interplay of heat, mass and momentum transfer in a microreactor when bubbles form on the catalytic surface using 2Dsimulations. The effect of bubbles on the endothermic process of Aqueous-Phase Reforming of a $10 \mathrm{wt} \%$ glycerol solution was evaluated in terms of conversion and temperature profile in the reactor. This study highlights the impact of bubbles not only on transport phenomena, but also, and ultimately, on the reactor performance.
\end{abstract}

Adapted from:

Ripken, R. M.; Wood, J.A.; Gardeniers, J. G. E.; Le Gac, S., Aqueous-Phase Reforming in a microreactor: the role of surface bubbles Submitted 2019 


\subsection{Introduction}

Many industrial chemical processes comprise one or multiple catalytic reactions. For large scale production, heterogeneous catalysis is preferred, in which a solid phase catalyst is used while reactants, products and solvent are in the gaseous and or/liquid phase. In some catalytic reactions, such as Aqueous-Phase Reforming (APR), ${ }^{1}$ gaseous products $\left(\mathrm{CO}, \mathrm{CO}_{2}\right.$, and $\left.\mathrm{H}_{2}\right)$ form as bubbles on the catalytic surface in the liquid reaction mixture, resulting in a three-phase system. The interplay of the different transport phenomena in such multiphase systems is relatively complex due to the differences in the physical properties of the fluids and the competing effects in transport phenomena, which altogether impacts the resulting chemical conversion and reactor performance.

Microfluidics is an ideal platform to study heat and mass transfer in such multiphase systems..$^{2-5}$ Not only offer these miniaturized systems accurate fluid control, but also they exhibit excellent heat and mass transfer properties as a result of their large surface-to-volume ratio. ${ }^{6-8}$ In microfluidic systems, the solid catalyst can be introduced as a packed bed, ${ }^{9-10}$ a monolithic phase (foam), ${ }^{11-12}$ or a thin-layer on the microchannel wall. ${ }^{13-14}$ Furthermore, and particularly, catalytic reactions suffering from slow kinetics benefit from the large catalytic surface area offered by microreactors.

However, the presence of bubbles on the catalytic surface can be highly undesirable, particularly in a microreactor, as they block the catalytic surface, preventing further reaction, obstruct liquid flow and also possibly negatively impact the temperature profile. At the same time, bubbles are no-shear interfaces, so that they can promote mixing and/or reduce drag forces (if no surfactant is added to the system) by providing effective slip. ${ }^{15-16}$ Typically, slip is quantified by a slip length, $b$, expressed as:

$$
b=\delta\left(\frac{\mu_{l}}{\mu_{g}}-1\right) \cong \delta \frac{\mu_{l}}{\mu_{g}}
$$

where $\delta$ is the thickness of the gas layer, and $\mu$ the viscosity of the liquid $(l)$ or gas $(g) .{ }^{17}$ Due to the large viscosity difference between gases and liquids, the slip length for bubbles can be in the order of micrometers. As microreactors have dimensions in the same order of magnitude, such a slippery interface near or on a catalytic surface cannot be neglected. ${ }^{18}$ 
As a result of the slip velocity, convection is locally increased, which is also influencing transport phenomena. ${ }^{19}$ In previous work, Karatay et al. studied the dissolution of protruding gas bubbles in liquid in a microreactor operating under laminar flow conditions. ${ }^{20}$ In that work, an array of elliptical or hemispherical bubbles was considered, forming a bubble mattress, which shares similarities with the case where bubbles are formed as products of a reactions such as AqueousPhase Reforming (APR). Depending on the exact bubble geometry, mass transport was enhanced in the channel due to the slippage at the gas/liquid interface.

Building on that work, ${ }^{20}$ here, we investigated the effect of a bubble mattress on the momentum, heat and mass transport to and from a catalytic surface, using COMSOL Multiphysics $5.3 \mathrm{a}^{21}$ as a 2D-numerical simulation. Specifically, APR of glycerol was considered as a model reaction. APR is an endothermic reaction, so that bubbles can play an important role in terms of the thermal properties, potential slip and loss of catalytic surface area. The transport phenomena (momentum, thermal and mass) and resulting reactor conversion were evaluated as a function of the bubble characteristics (length, protrusion angle, and catalytic surface coverage) at two temperatures to explore the possible impact of bubbles on the microreactor performance.

\subsection{Materials and methods}

\subsubsection{D microreactor geometry}

A 2D-model was considered to evaluate the momentum, heat and mass transfer in a catalytic microreactor, in which bubbles grow on a solid catalytic surface (Figure 4.1). This 2D-geometry is derived from the silicon/glass microfluidic device described in Chapter 3. ${ }^{22}$ Briefly, a 250- $\mu \mathrm{m}$ deep fluidic channel was created into a silicon substrate and covered by a glass top-layer. This microchannel was considered as infinitely wide. A $10-\mu$ m thick $\mathrm{TiO}_{2}$ layer on the silicon acted as a model catalyst. The catalytic reaction occurred at this boundary, where only external mass transport was taken into account. The device was heated from the silicon side using an external $\mathrm{MgO}$ ceramic heater. Heat was allowed to dissipate either through the glass cover or via the inand outlets; the remainder of the geometry was considered thermally insulated. 


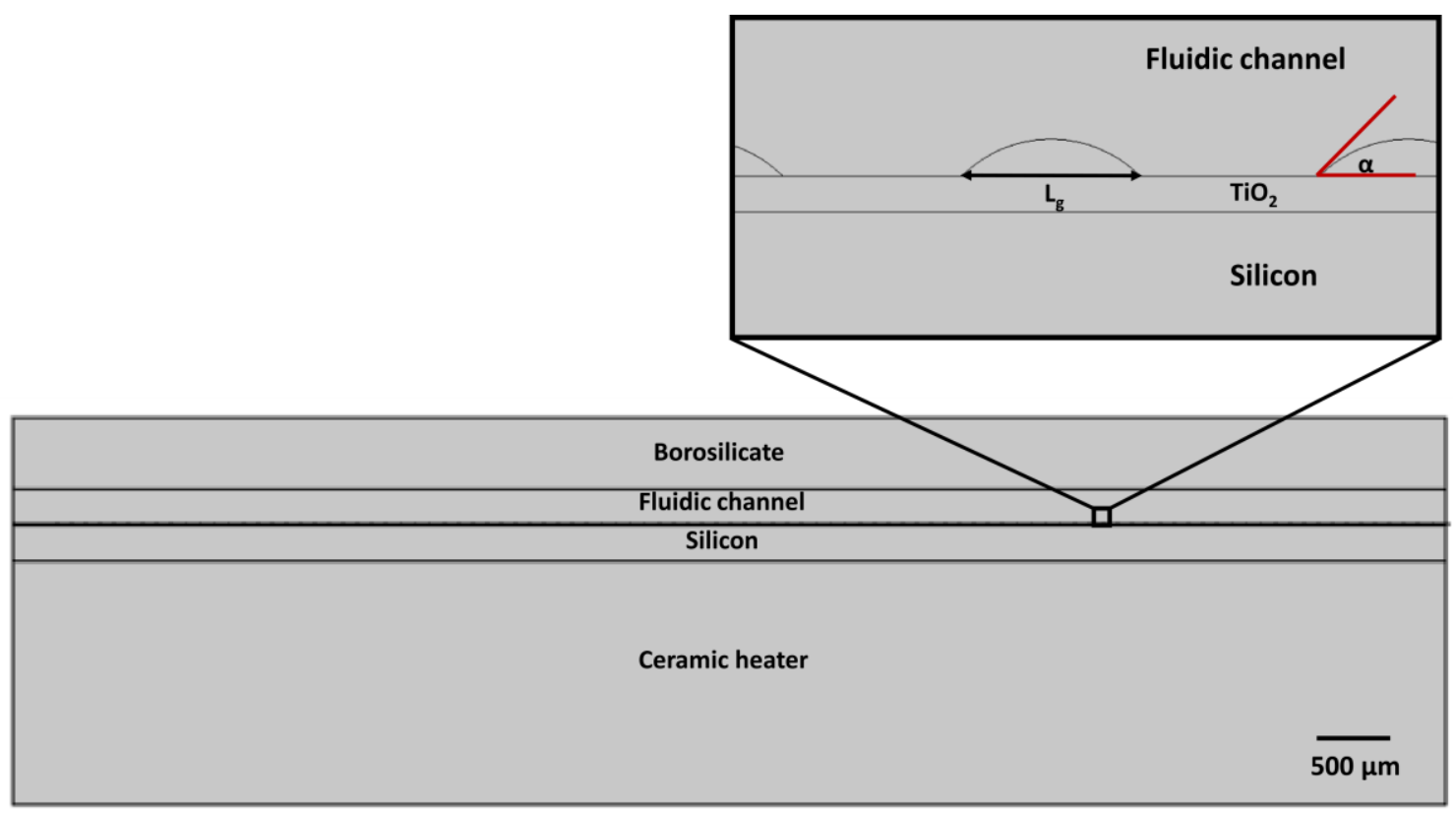

Figure 4.1 Schematic representation of the 2D-microreactor geometry considered for the COMSOL simulations, the different materials for each layer being indicated. The temperature is regulated from the silicon side using an external ceramic heater. The characteristic bubble length and protrusion angle are indicated with $L_{g}$, and $\alpha$, respectively.

A bubble mattress with hemispherical/elliptical $\mathrm{H}_{2}$ bubbles, depending on the protrusion angle, was modeled on the $\mathrm{TiO}_{2}$ surface as products of the catalytic reaction. These bubbles were described by their characteristic length $\left(L_{g}\right)$, their protrusion angle into the liquid $(\alpha)$, and the fraction of the catalytic surface covered by the bubbles $\left(\varepsilon_{B}\right)$, and all these parameters were varied in the simulations (See Table 4.1). A slip boundary condition was imposed on the bubble/liquid interface, and a no-slip boundary condition on the channel walls. The bubbles were treated as nondeformable and at a fixed position in the microchannel.

\subsubsection{Catalytic model reaction}

Aqueous-Phase Reforming (APR) was considered as a model reaction. In APR, oxygenated carbohydrates, such as alcohols and sugars, with a 1:1 C:O ratio, herein referred to as biomass substrates, are reformed into $\mathrm{CO}_{2}$ and $\mathrm{H}_{2}$ in two main steps. ${ }^{1,23}$ First, the biomass substrate is cracked into $\mathrm{CO}$ and $\mathrm{H}_{2}$, of which $\mathrm{CO}$ then reacts in the water-gas-shift reaction to form $\mathrm{CO}_{2}$ and additional $\mathrm{H}_{2}$. Per definition, both reactions take place in the liquid phase on the same catalytic surface, which commonly consists of a noble metal on an oxide. ${ }^{23}$ Typically, APR is performed at 
$550 \mathrm{~K}$, as the reaction is endothermic, so that a 30 bar pressure must be applied to keep the reaction mixture in the liquid phase. ${ }^{23}$ Here, a $10 \mathrm{wt} \%$ glycerol aqueous solution was considered as the biomass model substrate, and APR was modeled with first order reaction kinetics (See Appendix A4.2).

\subsubsection{Governing equations}

The fluid dynamics was evaluated by solving the steady-state Navier-Stokes equation for compressible fluids (Eq. 4.2), and the continuity equation (Eq. 4.3).

$$
\begin{aligned}
& \rho(\vec{u} \cdot \nabla) \vec{u}=-\nabla P+\nabla \cdot \mu\left[(\nabla \vec{u})+(\nabla \vec{u})^{T}\right]+\overrightarrow{f_{g}} \\
& \nabla \cdot(\rho \vec{u})=0
\end{aligned}
$$

with $\rho$ the density and $\mu$ the viscosity of the liquid phase, $\vec{u}$ the average mean velocity, $P$ the pressure, and $\overrightarrow{f_{g}}$ the volume force due to gravity (buoyancy).

The heat transfer, as described by equations 4.4 and 4.5, was scaled to the inlet and heater temperature, which were $293.15 \mathrm{~K}$ and $523.15 \mathrm{~K}$ or $725 \mathrm{~K}$, respectively.

$$
\rho C_{p} \vec{u} \cdot \nabla T+\nabla \cdot \vec{q}=0
$$

with:

$$
\vec{q}=-k \nabla T
$$

where $C_{p}$ is the heat capacity, $T$ the temperature, $\vec{q}$ the heat flux and $k$ the thermal conductivity.

The concentration profile in the bulk of the microchannel was computed by solving the convectiondiffusion equation, while neglecting thermodiffusion effects:

$$
\nabla \cdot\left(-D_{G l y} \nabla c_{G l y}+\vec{u} c_{G l y}\right)=0
$$

were $D_{G l y}$ and $c_{G l y}$ indicate the diffusivity constant and concentration of glycerol. During the simulations, the bubble length, protrusion angle and the catalytic surface coverage were varied to study their influence on the momentum and the heat and mass transfer, as summarized in Table 4.1, considering two temperature values (525 and $725 \mathrm{~K}$ ). Temperature-dependent physical 
properties were included, as those can have a large impact in this system. Appendix A4.1 provides a list of the expressions and corresponding references.

Table 4.1: Parametric sweep values: the bubble characteristics and heater temperature were varied to study their influence on the momentum, heat and mass transfer.

\begin{tabular}{ll}
\hline Parameter & Values \\
\hline Protrusion angle $\alpha\left[^{\circ}\right]$ & $10 ; 45 ; 90$ \\
Characteristic gas bubble length $L_{g}[\mu \mathrm{m}]$ & $20 ; 100$ \\
Fraction of the catalytic surface covered by bubbles $\varepsilon_{B}$ & $0.25 ; 0.5 ; 0.75$ \\
Heater temperature $T_{H}[\mathrm{~K}]$ & $525 ; 725$ \\
\hline
\end{tabular}

At low protrusion angles (below $45^{\circ}$ ) positive slip lengths are expected, and negative slip at higher angles. ${ }^{20}$ The low temperature value $(525 \mathrm{~K})$ corresponds to a typical APR reaction temperature, ${ }^{1}$ whereas the upper temperature limit $(725 \mathrm{~K})$ refers to the maximum temperature, at which our microreactor can operate. ${ }^{22}$

\section{Dimensionless Variables}

The system geometry was scaled to the channel height, as well as other domain variables, for the purpose of numerical stability and to understand the relative contribution of various transport mechanisms. The dimensionless forms of the domain variables are as follows:

$$
\bar{u}=\frac{\vec{u}}{u_{0}} \quad \bar{T}=\frac{T-T_{0}}{T_{H}-T_{0}} \quad \bar{c}=\frac{c_{\mathrm{Gly}}}{c_{0}}
$$

The dimensionless value of pressure is arbitrary, since all liquid properties are pressureindependent. $u_{0}$ was $1.25 \mathrm{~mm} . \mathrm{s}^{-1}, T_{0}$ was $293.15 \mathrm{~K}$, and $\mathrm{c}_{0}$ was $1.09 \mathrm{~mol} . \mathrm{L}^{-1}$. 


\subsubsection{Boundary conditions}

\section{Momentum transport (velocity/pressure)}

The dimensionless inlet fluid velocity was set at a constant value of 1 , so that the inlet velocity profile was fully developed before entering the fluidic channel at the inlet with an average velocity of $u_{0}$. The bubble/liquid interface was treated as no-shear, since there were no surfactants or other effects expected to reduce the interface to no-slip, ${ }^{17,20}$ which was considered as the best-case scenario for a microsystem with bubbles. The top wall of the microchannel and the catalytic surface were treated as no-slip and the outlet was set as a constant pressure outflow condition.

\section{Energy transport (temperature)}

The bottom of the ceramic heater was fixed at a specific temperature, assuming that the maximum power output of the heater was sufficient to maintain this temperature despite any loss to the environment or from the endothermic reaction. The inlet fluid temperature was set at $293.15 \mathrm{~K}$, and the heat of reaction taken into account at the interface of the fluid and the catalytic surface. The top of the glass layer was treated as a heat flux at a constant ambient temperature of 293.15 $\mathrm{K}$, using a heat transfer coefficient of $10 \mathrm{~W} \cdot \mathrm{m}^{-2} \cdot \mathrm{K}^{-1}$, which is a typical value for natural convection. ${ }^{24-25}$ Continuity of temperature was assumed for all other internal boundaries.

\section{Mass transport (concentration)}

The concentration of glycerol in the microchannel was fixed at an arbitrary constant concentration (dimensionless value of 1) at the channel inlet, and assumed to be sufficiently low to apply Fick's law. At the catalyst surface, a first order reaction rate model was applied with a temperaturedependent rate constant introduced via an Arrhenius expression (Appendix A4.2). ${ }^{26}$ A dimensionless rate constant (Damköhler number) could be calculated based on the characteristic dimensions of the reactor and rate constant at the reference temperature. For the bubble/liquid interface and top wall of the microchannel a no-flux boundary condition was prescribed. The outlet of the system was treated as a Neumann condition (zero normal gradient in concentration). 


\section{Simulation details}

The aforementioned equations were solved numerically for steady-state profiles using the finite element method in COMSOL Multiphysics 5.3a ${ }^{\text {TM}} .{ }^{21}$ For the temperature and the concentration, $2^{\text {nd }}$ order Lagrange polynomials were used and for the velocity/pressure a P2-P1 basis was used. Mesh independency was confirmed based on successive refinement and resolution for the smallest bubble size and coverage considered.

\subsection{Results and discussion}

\subsubsection{Velocity, temperature and concentration profiles}

First, the velocity and temperature profiles, and the conversion were studied in a bubble-free microfluidic channel. The velocity profile was found to be consistent with a laminar flow profile and a no-slip boundary condition at the microchannel wall. Figure 4.2 depicts the temperature (A.) and glycerol concentration profiles (B.) in the 2D-microreactor for the endothermic APR reaction of glycerol at $525 \mathrm{~K}$. Further downstream (increasing x.L $\mathrm{L}^{-1}$ ), the glycerol concentration decreased in the channel, as a result of the APR reaction. As the reaction is endothermic, the temperature in the channel decreased in the flow direction. The average dimensionless temperature at the outlet

A.

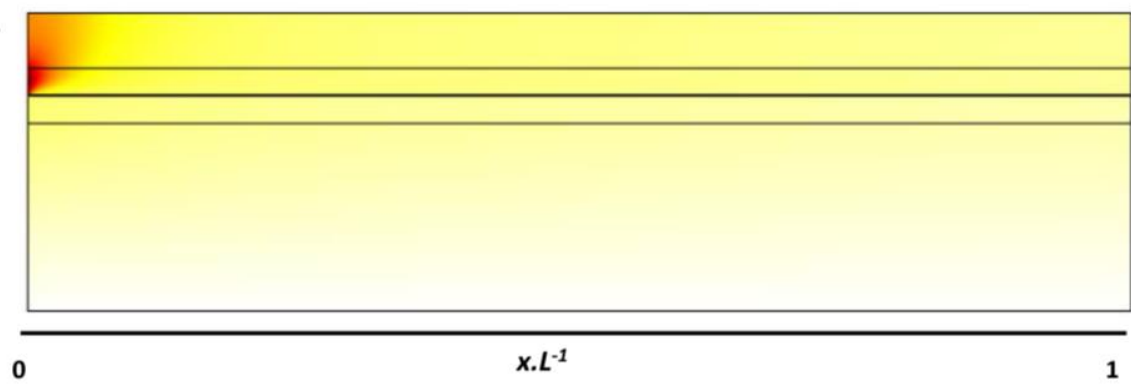

$\bar{T}$
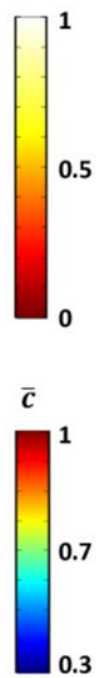

Figure 4.2 Dimensionless temperature profile over the entire microfluidic device (A.) and dimensionless glycerol concentration profile from the inlet to half-way in the fluidic channel (B.) in the absence of bubbles and at a heater temperature of $525 \mathrm{~K}$. 


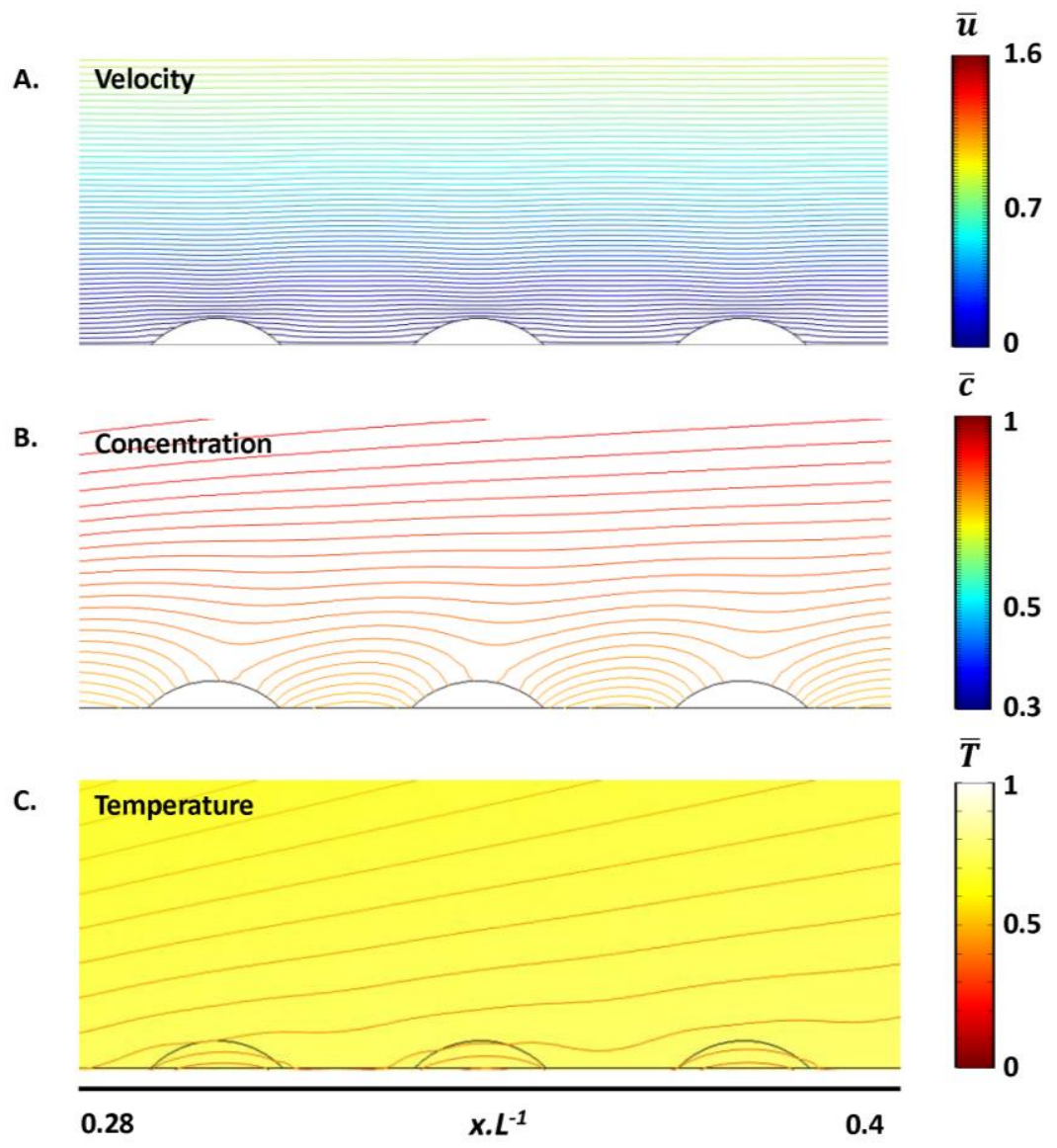

Figure 4.3 Dimensionless velocity (A.), glycerol concentration (B.) and temperature profiles (C.) at the inlet of the microfluidic channel around the first three bubbles ( $L_{g}=20 \mu \mathrm{m} ; \alpha=45^{\circ}$ and $\left.\varepsilon_{B}=0.5\right)$ when a temperature of $525 \mathrm{~K}$ was applied.

of the device was 0.84 , which corresponds to a physical temperature of $488 \mathrm{~K}$, whereas the average outlet conversion reached 0.56 .

The same analysis was performed with a bubble mattress $\left(L_{g}=20 \mu \mathrm{m} ; \alpha=45^{\circ}\right.$ and $\left.\varepsilon_{B}=0.5\right)$ at the same heater temperature. Figure 4.3 depicts the velocity, concentration and temperature profiles around the first three bubbles at the inlet of the fluidic channel. The average conversion determined at the outlet of the microreactor in the presence of bubbles was 0.46 , which is lower than in the absence of bubbles.

From a global perspective, the velocity, temperature, and concentration profiles seemed to be very similar to the bubble-free case. However, when considering the liquid/bubble interface, the velocity contour lines were much closer at the top of the bubble than near the channel wall, which 
indicates a steeper velocity gradient at the gas/liquid interface, due to the no-shear surface of the bubbles (Figure 4.3A.). The concentration profile (Figure 4.3B.) can be related to the position of the bubbles, since no reaction can occur either on the bubble or on the catalytic surface covered by bubbles.

The temperature profile (Figure 4.3C.) was found to be almost uniform across the channel height, with only a small temperature gradient near the bubble/liquid interface. The lateral temperature gradient was very small, which can be explained by the relatively high thermal conductivity of hydrogen compared to other gases, which are more insulating. ${ }^{27}$ The slightly higher temperature in the bubble than in the fluid may be counter-intuitive. However, since APR is an endothermic reaction, heat is only extracted at the catalytic surface area not covered by bubbles. Therefore, the temperature was lower at the catalytic surface than in the bubbles. Furthermore, the presence of a slip velocity at the bubble/liquid interface could not compensate for the temperature gradient resulting from the reaction. Altogether, while the temperature profile in the microchannel was found to be determined by the endothermic character of the reaction, heat transfer through the catalytic layer was most critical for the temperature profile.

\subsubsection{Effect of the bubble parameters on the conversion}

To study how the presence of bubbles affects the chemical conversion, a parametric sweep was run by varying the bubble length, protrusion angle and catalytic surface coverage. The conversion was evaluated at four positions in the fluidic channel. As illustrated on Figure 4.4, bubbles negatively affected the conversion, both at 525 and $725 \mathrm{~K}$, and the higher the temperature, the higher the glycerol conversion, as predicted by the Arrhenius equation.

The effect of the bubble characteristics on the conversion was investigated in more detail. The conversion was normalized to the available catalytic surface area $\left(1-\varepsilon_{B}\right)$ and plotted as a function of the same parameter, to determine how effectively the catalytic surface was used.

Figure 4.5 compares two specific scenarios, with bubbles of $20 \mu \mathrm{m}$ in length and a protrusion angle of $10^{\circ}$ (circle symbols), and bubbles of $100 \mu \mathrm{m}$ in length and a protrusion angle of $90^{\circ}$ (square symbols). In general, the bubble characteristics (length and protrusion angle) were found to only influence the conversion rate at a high catalytic surface coverage by the bubbles (high $\varepsilon_{B}$ ). For 1- 


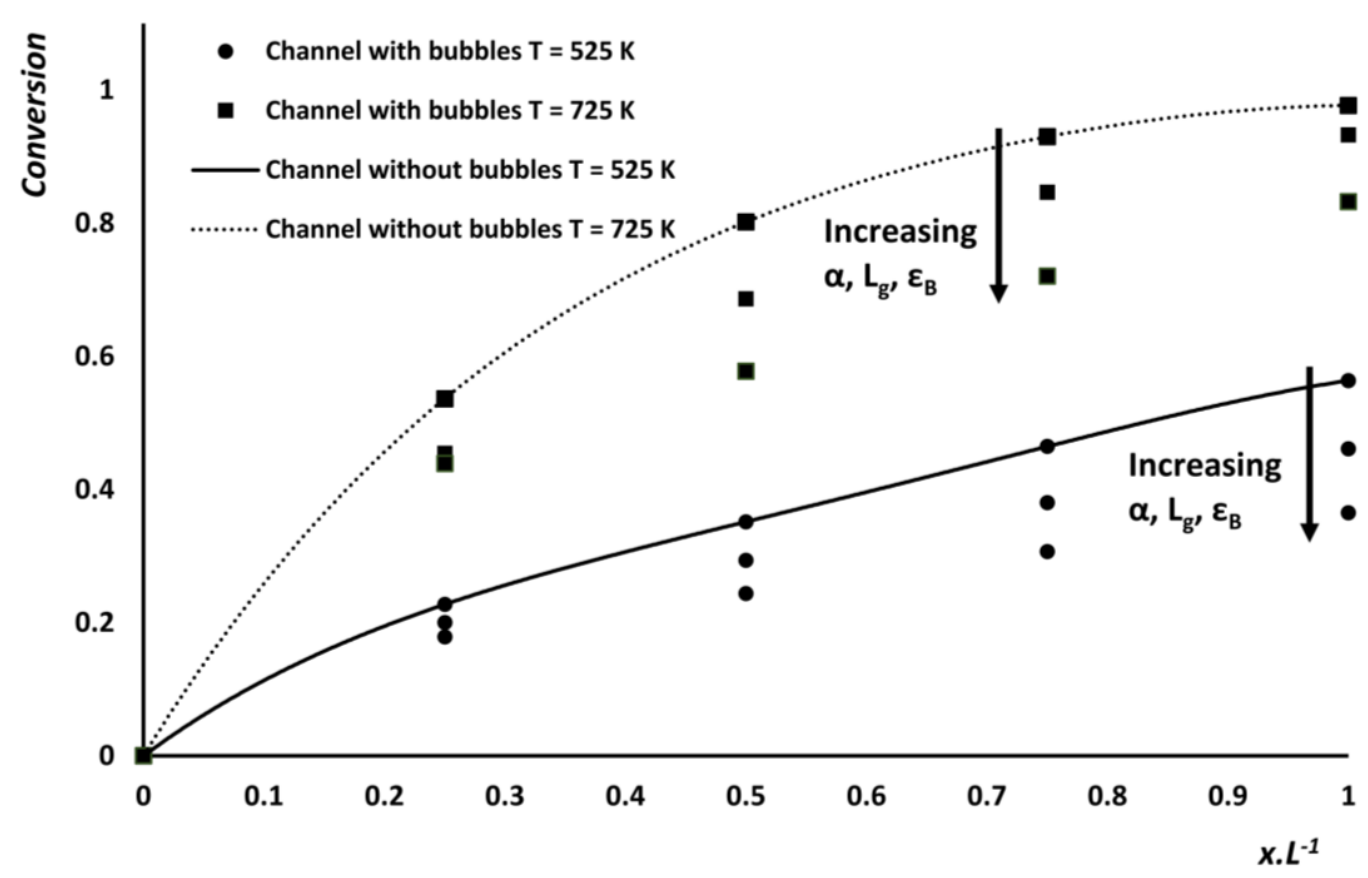

Figure 4.4 Chemical conversion as a function of the position in the fluidic channel for different bubble mattress characteristics. Squares ( $\boldsymbol{(})$ and triangles ( $\mathbf{A})$ indicate heater temperatures of 725 and $525 \mathrm{~K}$, respectively. The curves depict the conversion in absence of bubbles.

$\varepsilon_{B}=0.25$, the highest conversion rate was achieved for shorter and flatter bubbles $\left(L_{g}=20 \mu \mathrm{m} ; \alpha\right.$ $=10^{\circ}$ ) for both considered temperatures. For such bubbles, a higher degree of slip is expected, so that they are less detrimental for the conversion than larger bubbles.

As shown in both Figures 4.5A. and 4.5B., the conversion per available unit of catalytic surface area decreased when increasing the available catalytic surface area, which is likely due to a temperature effect: when a larger catalytic surface area is available to the liquid, more heat was utilized for the reaction, which in turn results in a lower temperature in the channel, and in a lower conversion.

\subsubsection{Temperature}

Finally, the temperature profile was evaluated in the microchannel for different bubble characteristics and applied temperatures. Figure 4.6 depicts the temperature as a function of the $x$ position in the channel, for both heater temperatures, measured at half-height of the fluidic channel. For sake of clarity, only non-overlapping data are shown in Figure 4.6. Surprisingly, the 

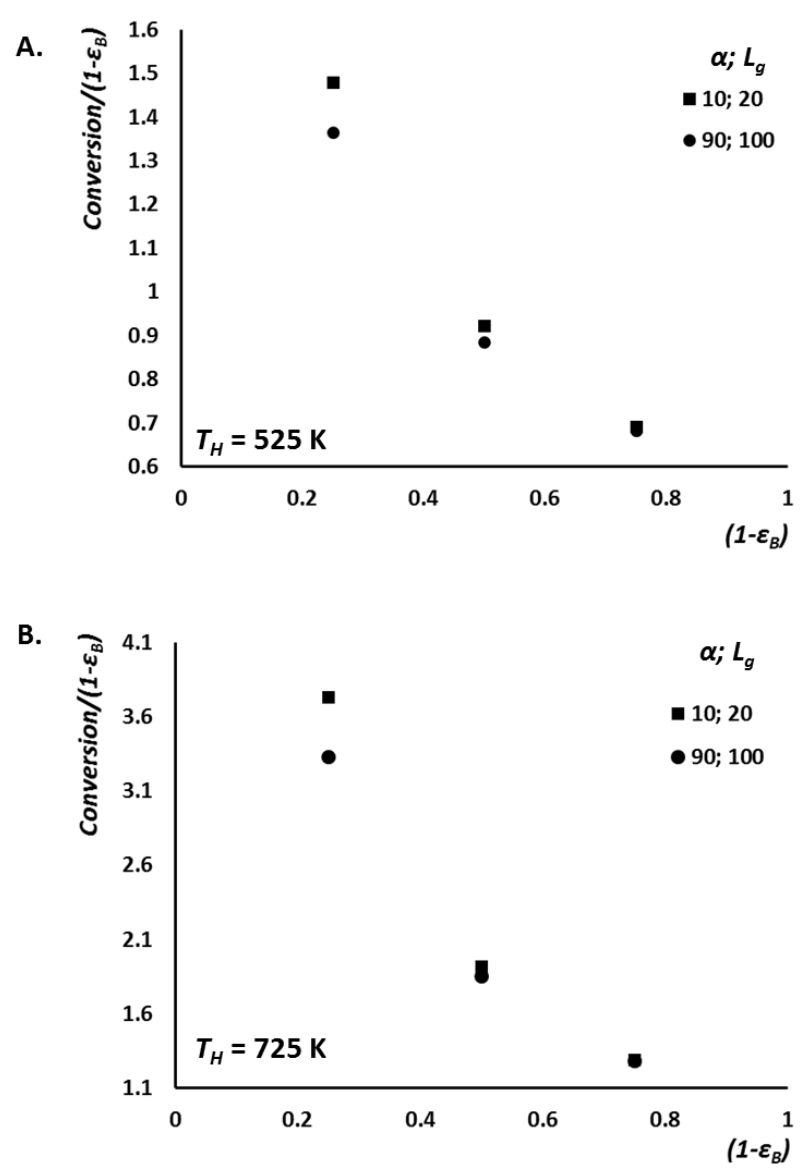

Figure 4.5 Conversion normalized to the available catalytic surface $\left(1-\varepsilon_{B}\right)$ as a function of the available catalytic surface $\left(1-\varepsilon_{B}\right)$, for a heater temperature of $525 \mathrm{~K}$ (A.) and $725 \mathrm{~K}$ (B.). Bubbles of $20 \mu \mathrm{m}$ in length with a protrusion angle of $10^{\circ}$ and bubbles of $100 \mu \mathrm{m}$ in length and a protrusion angle of $90^{\circ}$ are depicted by $(\mathbf{})$ or $(\bullet)$ symbols, respectively.

temperature was higher in the presence of bubbles, for all considered bubble configurations compared to a bubble-free situation. The highest temperature was obtained for bubbles characterized by $\alpha=90^{\circ}$ and $L_{g}=100 \mu \mathrm{m}$, and a catalytic surface coverage $\varepsilon_{B}$ of 0.75 .

These results are counter-intuitive, since a higher catalytic surface coverage $\varepsilon_{B}$ would be expected to correspond to a larger insulating layer, which should therefore prevent heat transfer. However, the temperature profile as depicted in Figure 4.6 supports the trends observed for the reaction conversion (Figure 4.4). For a lower conversion rate, less heat was used for the reaction, which overall resulted in a higher temperature in the channel. Altogether, the temperature in the fluid was mainly dictated by the chemical conversion, for which the heat transfer through the catalytic layer was limiting. Furthermore, in this work, we considered hydrogen bubbles, which have a relatively 
A
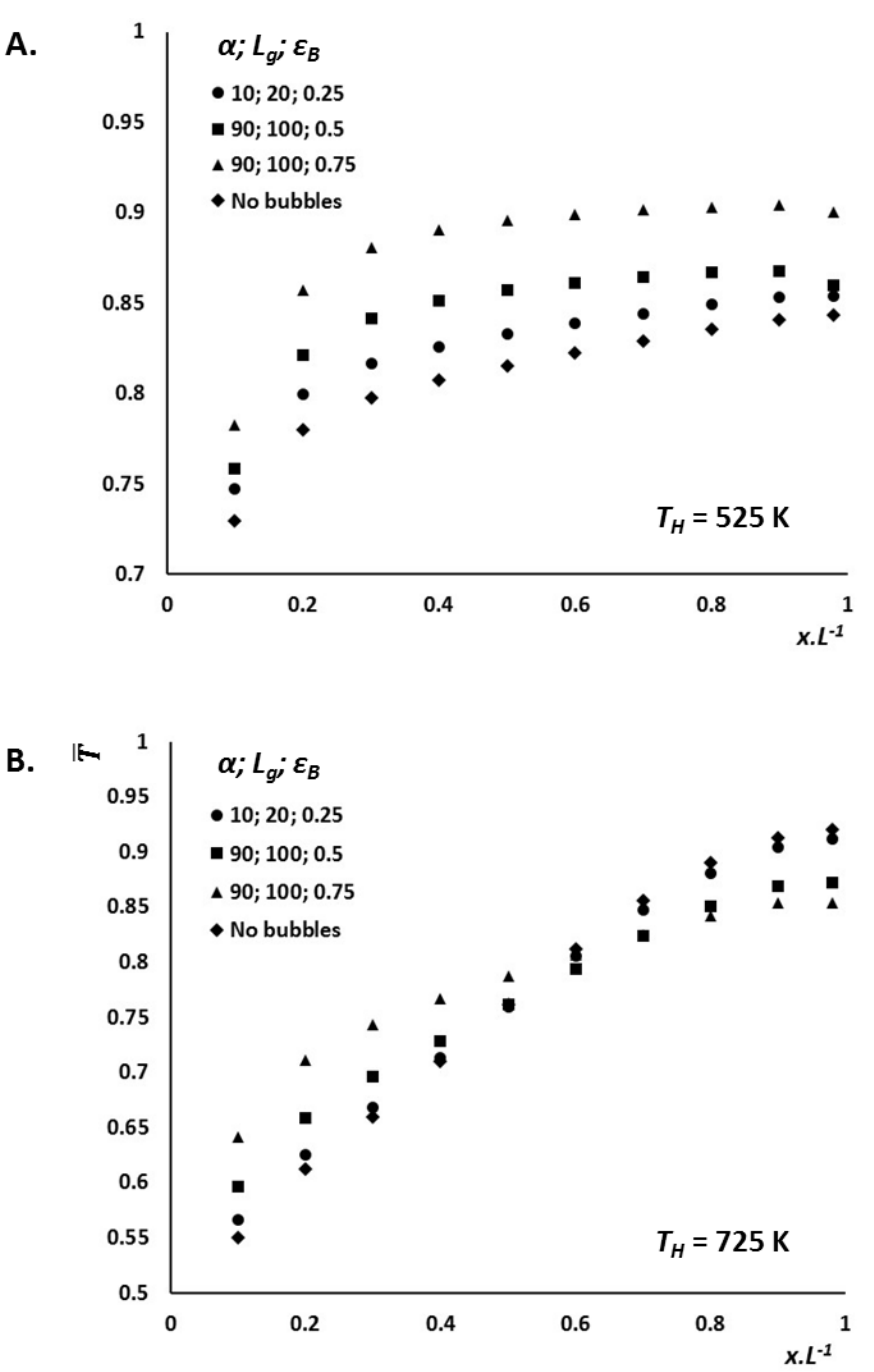

Figure 4.6 Dimensionless temperature profile at the half-height of the fluidic channel as a function of the position in the channel for various bubble characteristics and for a heater temperature of $525 \mathrm{~K}$ (A.) and $725 \mathrm{~K}$ (B.).

high thermal conductivity compared to other gases. ${ }^{27}$ Thus, the effect of $\mathrm{H}_{2}$ bubbles on the heat transfer was relatively smaller than for more insulating gases.

Next, three different temperature regions could be distinguished, which were mainly determined by the available catalytic surface coverage $\left(1-\varepsilon_{B}\right)$, since the bubble protrusion angle and length did not significantly affect the temperature at the half-height of the fluidic channel. At an applied temperature of $525 \mathrm{~K}$, the relation between the temperature and the bubble mattress characteristics $\left(L_{g}, \alpha\right.$, and $\left.\varepsilon_{B}\right)$ was straightforward: the temperature in the channel increased with the catalytic surface coverage $\varepsilon_{B}$. 
A similar trend was observed for $x . L^{-1}$ smaller than 0.6, and an applied temperature of $725 \mathrm{~K}$. Further downstream in the channel, this situation was reversed, and higher temperatures were found in bubble-free channels. In absence of bubbles, full chemical conversion was achieved at this specific location in the channel, so that no more heat was used for the endothermic APR reaction, and all the energy was transferred to the fluid. As full conversion was achieved at a later location in the presence of bubbles, heat was still used for the chemical reaction, so that the temperature became lower in the channel.

Overall, heat and mass transfer were found to depend on the degree of conversion, which was in turn determined by the catalytic surface coverage $\varepsilon_{B}$. As the herein considered reaction is endothermic, the conversion and, more indirectly, the catalytic surface coverage dominate the temperature profile. Overall, the slip velocity on the no-shear bubble/liquid interface was found to not play a significant role in the transport phenomena. In systems where gas bubbles inevitably form on the catalytic surface, applying a higher heater temperature can aid to counter act the negative effects of the bubbles. At higher temperature, diffusion constants also become higher, which promotes mass transfer towards and from the catalytic surface.

\subsubsection{Model relevance}

Several aspects must be considered when translating the results from this study to an actual catalytic microreactor. First of all, in this work, a 2D-model was used, with channels having an infinite width, while in an experimental microreactor, the fluid is also confined by side walls. As the microchannel width is very comparable to the channel depth, gradients caused by the presence of the side-walls cannot be neglected.

The model could also be extended to include the bubble nucleation and growth. Product gases are expected to dissolve into the liquid until the maximum solubility is reached, as described by Henry's Law. Once the liquid is saturated, bubbles are more likely to nucleate on rough surfaces, such as the catalytic layer or on channel defects. During the bubble growth, a gas depletion layer forms around the bubble, which increases the gas flux from the bulk liquid towards the bubble. ${ }^{28}$ In addition, the presence of temperature gradients at the bubble/liquid interface can lead to 
variations in the surface tension at this interface, which may in turn result in additional surface flows, also known as the Marangoni effect. ${ }^{29}$

Furthermore, the geometry and contact area of the bubble on the catalytic surface depend on the surface properties of the catalyst. In this study, we considered bubbles with a protrusion angle $\alpha$ up to $90^{\circ}$. However, bubbles can also adopt more spherical shapes, have much smaller contact areas with the catalyst, and therefore do not cover the catalytic surface as much as hypothesized in this work. Furthermore, spherical bubbles also possibly give rise to corner flows, which would enhance mixing. Altogether, spherical bubbles are expected to limit the conversion to a lesser extent than bubbles considered in this study, which will be the topic of Chapter 5.

\subsection{Conclusion}

Transport phenomena in multiphase catalytic microreactors, where bubbles grow on a catalytic surface are relatively complex. Here, we studied the momentum, heat and mass transfer in a multiphase microreactor using a 2D-COMSOL simulation for different bubble characteristics (length, protrusion angle and overall catalytic surface coverage), and APR as a model reaction. As expected, the presence of bubbles decreased the overall chemical conversion, as they partially blocked the catalytic surface: the longer the bubbles, the lower the conversion. Although bubbles have a no-shear surface, the existence of a slip velocity was not sufficient to compensate for the loss of catalytic surface. For the considered endothermic APR reaction, the temperature in the fluidic channel was reduced, which in turn affected the diffusivity of the reactants to the catalytic surface. Altogether, this work highlights the importance of understanding the effect of bubbles on the interconnected transport phenomena in microreactors.

\section{References}

1. Cortright, R. D.; Davda, R. R.; Dumesic, J. A., Hydrogen from catalytic reforming of biomass-derived hydrocarbons in liquid water. Nature 2002, 418 (6901), 964-967.

2. Worner, M., Numerical modeling of multiphase flows in microfluidics and micro process engineering: a review of methods and applications. Microfluidics and Nanofluidics 2012, 12 (6), 841-886. 
3. Gomez-Pastora, J.; Gonzalez-Fernandez, C.; Fallanza, M.; Bringas, E.; Ortiz, I., Flow patterns and mass transfer performance of miscible liquid-liquid flows in various microchannels: Numerical and experimental studies. Chem. Eng. J. 2018, 344, 487-497.

4. Stafford, J., Principle-based design of distributed multiphase segmented flow. Int. J. Heat Mass Transfer 2016, 100, 508-521.

5. Jimenez-Martinez, J.; Porter, M. L.; Hyman, J. D.; Carey, J. W.; Viswanathan, H. S., Mixing in a three-phase system: Enhanced production of oil-wet reservoirs by CO2 injection. Geophysical Research Letters 2016, 43 (1), 196-205.

6. Xu, B.-B.; Zhang, Y.-L.; Wei, S.; Ding, H.; Sun, H.-B., On-Chip Catalytic Microreactors for Modern Catalysis Research. ChemCatChem 2013, 5 (8), 2091-2099.

7. Munirathinam, R.; Huskens, J.; Verboom, W., Supported Catalysis in Continuous-Flow Microreactors. Adv. Synth. Catal. 2015, 357 (6), 1093-1123.

8. Porta, R.; Benaglia, M.; Puglisi, A., Flow Chemistry: Recent Developments in the Synthesis of Pharmaceutical Products. Organic Process Research \& Development 2016, 20 (1), 225.

9. Iliuta, I.; Hamidipour, M.; Schweich, D.; Larachi, F., Two-phase flow in packed-bed microreactors: Experiments, model and simulations. Chem. Eng. Sci. 2012, 73, 299-313.

10. He, W.; Fang, Z.; Zhang, K.; Tu, T.; Lv, N.; Qiu, C.; Guo, K., A novel micro-flow system under microwave irradiation for continuous synthesis of 1,4-dihydropyridines in the absence of solvents via Hantzsch reaction. Chem. Eng. J. 2018, 331, 161-168.

11. Zhu, L.; Fu Tan, C.; Gao, M.; Ho, G. W., Design of a Metal Oxide-Organic Framework (MoOF) Foam Microreactor: Solar-Induced Direct Pollutant Degradation and Hydrogen Generation. Adv. Mater. 2015, 27 (47), 7713-7719.

12. Chen, G.; Zhu, X.; Chen, R.; Liao, Q.; Ye, D.; Feng, H.; Liu, J.; Liu, M., Gas-liquid-solid monolithic microreactor with Pd nanocatalyst coated on polydopamine modified nickel foam for nitrobenzene hydrogenation. Chem. Eng. J. 2018, 334, 1897-1904.

13. Tanimu, A.; Jaenicke, S.; Alhooshani, K., Heterogeneous catalysis in continuous flow microreactors: A review of methods and applications. Chem. Eng. J. 2017, 327, 792-821.

14. D'Angelo, M. F. N.; Ordomsky, V.; Paunovic, V.; van der Schaaf, J.; Schouten, J. C.; Nijhuis, T. A., Hydrogen Production through Aqueous-Phase Reforming of Ethylene Glycol in a Washcoated Microchannel. ChemSusChem 2013, 6 (9), 1708-1716. 
15. Ou, J.; Perot, B.; Rothstein, J. P., Laminar drag reduction in microchannels using ultrahydrophobic surfaces. Physics of Fluids 2004, 16 (12), 4635-4643.

16. Bocquet, L.; Barrat, J. L., Flow boundary conditions from nano- to micro-scales. Soft Matter 2007, 3 (6), 685-693.

17. Vinogradova, O. I.; Dubov, A. L., Superhydrophobic textures for microfluidics. Mendeleev Commun. 2012, 22 (5), 229-236.

18. Ou, J.; Rothstein, J. P., Direct velocity measurements of the flow past drag-reducing ultrahydrophobic surfaces. Physics of Fluids 2005, 17 (10).

19. Bocquet, L.; Lauga, E., A smooth future? Nature Materials 2011, 10, 334.

20. Karatay, E.; Tsai, P. A.; Lammertink, R. G. H., Rate of gas absorption on a slippery bubble mattress. Soft Matter 2013, 9 (46), 11098-11106.

21. COMSOL AB COMSOL Multiphysics 5.3a, AB, COMSOL: Stockholm, Sweden, 2018.

22. Ripken, R. M.; Schlautmann, S.; Sanders, R. G. P.; Gardeniers, J. G. E.; Le Gac, S., Monitoring phase transition of aqueous biomass model substrates by high-pressure and hightemperature microfluidics. ELECTROPHORESIS 2019, 40 (4), 563-570.

23. Davda, R. R.; Shabaker, J. W.; Huber, G. W.; Cortright, R. D.; Dumesic, J. A., A review of catalytic issues and process conditions for renewable hydrogen and alkanes by aqueous-phase reforming of oxygenated hydrocarbons over supported metal catalysts. Applied Catalysis BEnvironmental 2005, 56 (1-2), 171-186.

24. Engineering ToolBox, Convective Heat Transfer. https://www.engineeringtoolbox.com/convective-heat-transfer-d_430.html.

25. Edge, E., Convective heat transfer coefficients table chart. 2019.

26. Adhikari, S.; Fernando, S. D.; Haryanto, A., Kinetics and Reactor Modeling of Hydrogen Production from Glycerol via Steam Reforming Process over Ni/CeO2 Catalysts. Chemical Engineering \& Technology 2009, 32 (4), 541-547.

27. Rumble, J. R., Handbook of Chemistry and physics. 99th ed.; CRC Press.

28. Lv, P.; Le The, H.; Eijkel, J.; Van den Berg, A.; Zhang, X. H.; Lohse, D., Growth and Detachment of Oxygen Bubbles Induced by Gold-Catalyzed Decomposition of Hydrogen Peroxide. Journal of Physical Chemistry C 2017, 121 (38), 20769-20776. 
29. Faghri, A.; Zhang, Y., 5 - SOLID-LIQUID-VAPOR PHENOMENA AND INTERFACIAL HEAT AND MASS TRANSFER. In Transport Phenomena in Multiphase Systems, Faghri, A.; Zhang, Y., Eds. Academic Press: Boston, 2006; pp 331-420.

30. Association, G. P. Physical properties of glycerine and its solutions. https://www.aciscience.org/docs/Physical_properties_of_glycerine_and_its_solutions.pdf.

31. Schott BOROFLOAT® 33 - 33 Thermal Properties. https://www.schott.com/d/borofloat/4d03b8f4-0583-4139-9841-952114e60dee/schott-data-sheetthermal-properties-borofloat-english-23112018.pdf.

32. Slifka, A. J.; Filla, B. J.; Phelps, J. M., Thermal Conductivity of Magnesium Oxide From Absolute, Steady-State Measurements. Journal of research of the National Institute of Standards and Technology 1998, 103 (4), 357-363.

33. Coquil, T.; Reitz, C.; Brezesinski, T.; Nemanick, E. J.; Tolbert, S. H.; Pilon, L., Thermal Conductivity of Ordered Mesoporous Titania Films Made from Nanocrystalline Building Blocks and Sol-Gel Reagents. The Journal of Physical Chemistry C 2010, 114 (29), 12451-12458.

34. te Riele, M. J. M.; Snijder, E. D.; van Swaaij, W. P. M., Diffusion Coefficients at Infinite Dilution in Water and in N-Methylpyrrolidone. Journal of Chemical \& Engineering Data 1995, 40 (1), 34-36.

35. Ripken, R. M.; Meuldijk, J.; Gardeniers, J. G. E.; Le Gac, S., Influence of the Water Phase State on the Thermodynamics of Aqueous-Phase Reforming for Hydrogen Production. Chemsuschem 2017, 10 (24), 4909-4913.

36. Iliuta, I.; Iliuta, M. C., Biosyngas Production in an Integrated Aqueous-Phase Glycerol Reforming/Chemical Looping Combustion Process. Industrial \& Engineering Chemistry Research 2013, 52 (46), 16142-16161. 


\section{A 4.1 Temperature dependency of physical properties}

Density

$$
\begin{array}{ll} 
& \text { Data obtained from: } \\
\rho=\frac{\rho_{H 2 O \_G L Y}\left(T *\left(T_{H}-T_{0}\right)+T_{0}\right)}{\rho_{H 2 O_{-} G L Y} T_{0}} & \operatorname{Ref}^{30}
\end{array}
$$

Thermal conductivity

The dimensionless temperature-dependent thermal conductivity was implemented according to:

$$
\bar{k}_{\text {material }}=\frac{k_{\text {material }}\left(T *\left(T_{H}-T_{0}\right)+T_{0}\right)}{k_{\text {borosilicate glass }}}
$$

with $1.12 \mathrm{~W} \cdot \mathrm{m}^{-1} \mathrm{~K}^{-1}$ for the thermal conductivity of borosilicate. ${ }^{31}$ The thermal conductivity of the other materials was extracted from the reference indicated in the table below, and, depending on the available data, either interpolated for temperature or linearly extrapolated.

\section{Data obtained from:}

$\begin{array}{ll}k_{\text {silicon }} & \operatorname{Ref}^{27} \\ \mathrm{k}_{\mathrm{MgO}} & \operatorname{Ref}^{32} \\ \mathrm{k}_{\mathrm{TiO} 2} & \operatorname{Ref}^{33} \\ \mathrm{k}_{\mathrm{H} 2 \mathrm{O} / \mathrm{Gly}} & \operatorname{Ref}^{30} \\ \mathrm{k}_{\mathrm{H} 2} & \operatorname{Ref}^{27}\end{array}$

Viscosity

$$
\eta=\frac{\eta_{H 2 O_{-} G L Y}\left(T *\left(T_{H}-T_{0}\right)+T_{0}\right)}{\eta_{H 2 O_{-} G L Y} T_{0}} \quad \quad \operatorname{Ref}^{30}
$$

Diffusivity

$$
D=\frac{D_{H 2 O_{-} G L Y}\left(T *\left(T_{H}-T_{0}\right)+T_{0}\right)}{D_{H 2 O_{-} G L Y} T_{0}} \quad \operatorname{Ref}^{34}
$$

\section{Data obtained from:}




\section{A4.2 Reaction data}

\section{Parameter}

Heat of reaction

Kinetic rate constant

Activation energy
Value $337.71\left[\mathrm{~kJ}^{\mathrm{mol}}{ }^{-1}\right.$ ]

$8135.5\left[\mathrm{~s}^{-1}\right]$

$60\left[\mathrm{~kJ} \cdot \mathrm{mol}^{-1}\right]$
Data obtained from:

$$
\begin{aligned}
& \operatorname{Ref}^{35} \\
& \operatorname{Ref}^{26} \\
& \operatorname{Ref}^{36}
\end{aligned}
$$




\title{
Towards controlled bubble nucleation in microreactors for enhanced mass transport
}

\begin{abstract}
The interplay of heat and mass transfer in a catalytic microreactor in which bubbles grow on the surface is highly complex. Depending on the contact angle and the location of the bubbles in the microchannel, transport phenomena can be enhanced, and, as a consequence, the conversion and the reactor efficiency are expected to increase. A promising approach to ultimately control transport phenomena in a catalytic gas/liquid/solid microreactor, is to direct the bubble size and nucleation. For this purpose, a microfluidic device was developed to direct the bubble nucleation with the help of hydrophobic micropits placed at well-defined locations along the microchannel. The nucleation of bubbles using either boiling or $\mathrm{CO}_{2}$ outgassing was studied in this device. It was observed that, instead of on the predefined positions, bubbles were formed on the defects on the side wall of the microchannel. In addition to experimental work, a 3D-numerical model in COMSOL Multiphysics 5.3a was developed to study the effect of these spherical bubbles on concentration gradients and chemical conversion in the microchannel, in the presence or absence of a catalytic layer.
\end{abstract}

Part of this chapter was adapted from:

Ripken, R. M.; Wood, J.A.; Schlautmann, S.; Guenther, A.; Gardeniers, J. G. E.; Le Gac, S., Controlled bubble nucleation in gas-liquid-solid catalytic microsystems for enhanced mass transport MicroTAS, Proceedings 2018

Ripken, R. M.; Schlautmann, S.; Wood, J.A.; Guenther, A.; Gardeniers, J. G. E.; Le Gac, S., Towards controlled bubble nucleation in microreactors for enhanced mass transport In preparation 


\subsection{Introduction}

In some heterogeneous catalytic reactions, gaseous products form as bubbles on the catalytic surface in a liquid reaction mixture. For example, in Aqueous-Phase Reforming (APR), $\mathrm{CO}, \mathrm{CO}_{2}$ and $\mathrm{H}_{2}$ are formed from oxygenated carbohydrates with a $\mathrm{C}: \mathrm{O}$ 1:1 ratio, such as sugars and polyalcohols. $^{1-3}$

At a first glance, the bubbles formed in APR may have an undesired effect, as they would block the catalytic surface for further reaction. In Chapter 4, the influence of bubbles on the momentum, heat and mass transfer in a microreactor was numerically investigated for APR of a $10 \mathrm{wt} \%$ glycerol aqueous solution. In that study, bubbles with hemispherical or elliptical shapes were considered with contact angles up to $90^{\circ}$. There, the conversion and the temperature profile in the microreactor were mainly determined by the catalytic surface coverage by the bubbles: the bubble protrusion angle did neither affect the conversion, nor the temperature profile.

However, depending on the (catalytic) surface properties, bubbles can adopt higher contact angles

and more spherical geometries to reduce their surface energy in an aqueous phase. ${ }^{4-5}$ Bubbles with a higher contact angle have a smaller contact area with the catalytic surface. Therefore, these bubbles have a higher tendency to be released from the surface due to convection, which is beneficial for the reaction at the catalytic surface.

The bubble contact angle can be manipulated by, for instance, an electric field in electrowetting on dielectrics (EWOD). ${ }^{6-7}$ Another possibility is to introduce roughness in the form of (hydrophobic) nano- or microstructures on a surface..$^{8-9}$ Gas is trapped in these small structures, which renders the surface more hydrophobic. For this purpose, hierarchically wrinkled $\mathrm{MoS}_{2}$ surface were fabricated by Jung et al, ${ }^{10}$ who reported that the amount of wrinkles affected the surface wettability and therefore the bubble contact angle: the more the wrinkling on the surface, the higher the bubble contact angle. ${ }^{11-12}$

During heterogeneous catalysis, such as APR, bubbles with high contact angles $\left(>90^{\circ}\right)$ are pinned either to the rough catalytic surface or on a defect on the microchannel wall. In this case, bubbles have a very small contact area with the catalytic surface. As a result of the no-shear bubble/liquid interface, a slip velocity occurs at this boundary. ${ }^{13-15}$ Consequently, both mixing ${ }^{16}$ and transport phenomena in the microreactor can be enhanced in the presence of this slip velocity, resulting in concentration gradients in the microchannel. Furthermore, corner flows near the spherical 
bubble/surface interface can also positively affect transport phenomena, as was numerically demonstrated for heat transport in a system in which the bubbles were pinned to a microcavity. ${ }^{17}$ To study, and ultimately control, transport phenomena in multiphase systems such as APR, controlling the contact angle and location of the bubble, as well as the bubble departure radius, is an interesting approach. It is important that the bubble departure radius, $R_{d}$, remains smaller than the microchannel diameter, otherwise the bubble would block the fluid flow. ${ }^{18-19}$ The bubble departure radius $\left(R_{d}\right)$ is derived from the ratio between buoyancy forces and surface tensional forces: ${ }^{20}$

$$
R_{d}=\left(\frac{3 R_{p} \sigma}{2 \Delta \rho g}\right)^{1 / 3}
$$

where $R_{p}$ is the radius of the microstructure on which the bubble is pinned, $\sigma$ the surface tension, $\Delta \rho$ the density difference between the liquid and the gas phase, and $g$ the gravitational constant. Therefore, by playing with the microstructure size, one can potentially control the bubble departure radius. Interestingly, the ratio between the buoyancy and surface tensional forces can also be influenced by the microstructure shape. This aspect has been investigated by Yamamoto et al, ${ }^{21}$ who demonstrated that by using saw-tooth shaped micropits the surface tensional forces can deliberately be increased compared to randomly shaped defects, so that the bubble can depart at smaller buoyancy forces.

By fabricating specific microstructures at defined spots in a microfluidic channel, the location for bubble nucleation can be controlled. ${ }^{22}$ However, to minimize the bubble departure radius, the microstructure size must be very small. Furthermore, the surface tension must be accurately tuned, for example by fluorocarbon deposition to render the surface more hydrophobic. ${ }^{22}$

In this chapter, a microfluidic device was designed and fabricated to include hydrophobic micropits to control the bubble nucleation in microreactors for gas/liquid/solid reactions, as used for APR. The performance of these micropits was tested using both boiling of water and $\mathrm{CO}_{2}$ outgassing and the resulting bubble patterns and pinning of the bubbles were evaluated. Furthermore, a 3D numerical model was built in COMSOL Multiphysics 5.3a, to qualitatively study the bubble influence on the momentum, substrate concentration gradients, and conversion of an arbitrary reaction in the microchannel in the presence or absence of a catalytic layer. Finally, the effect of the location of the catalytic layer with respect to the bubbles on the conversion was investigated. 


\subsection{Materials and methods}

\subsubsection{Microfluidic device}

In Chapter 3, a microfluidic device was fabricated to perform APR at high-pressure and-high temperature reaction conditions. This device consisted of silicon and glass, which is not suitable to conduct high resolution optical microscopy imaging. Therefore, to image the bubble nucleation, a microfluidic device with fully transparent fluidic channels was designed and fabricated (Figure 5.1). To direct bubble nucleation at well-defined locations in the microchannel, hydrophobic micropits were included in the bottom glass layer of the device (Figure 5.1B.).

A.

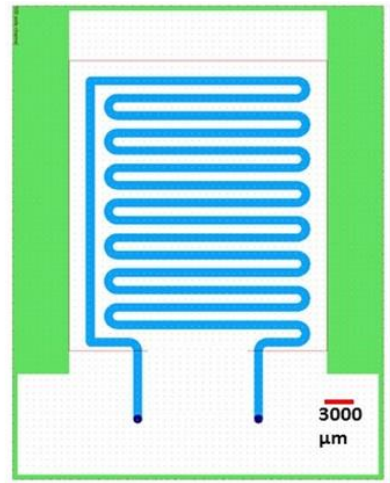

B.

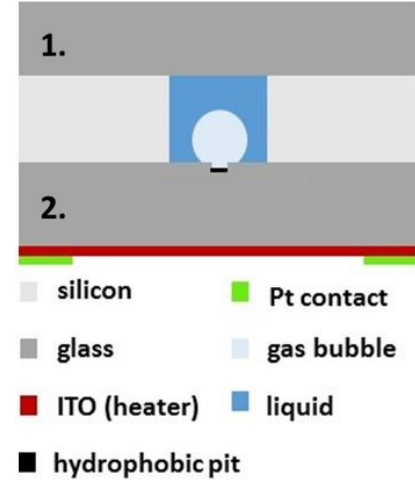

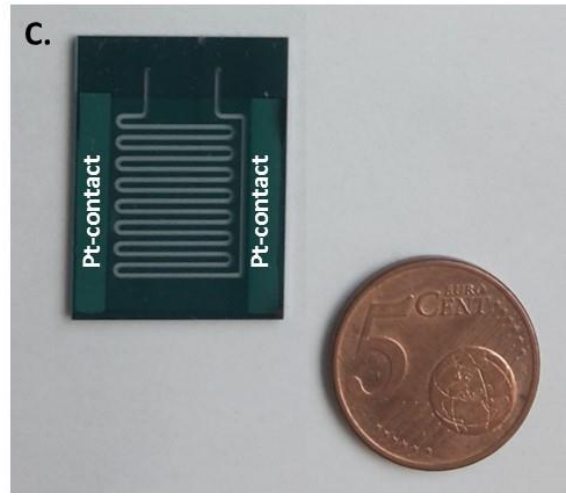

Figure 5.1 Microfluidic device for bubble nucleation experiments A. Design of the device (top view), with in light blue the fluidic channel, the Pt-contacts in green, and the in- and outlets in dark blue; B. Schematic representation of the cross-section of the device (not to scale), with the two glass layers numbered 1. and 2., the intermediate silicon layer in light gray, the fluidic channel with a bubble in blue and a hydrophobic micropit in black, C. Picture of the actual device with the Pt-contacts.

The device consisted of a glass-silicon-glass stack, with a 525- $\mu \mathrm{m}$ deep and $500-\mu \mathrm{m}$ wide meandering channel etched through the intermediate silicon substrate. On the back side of the bottom glass layer (numbered 2 in Figure 5.1B.), a 65-nm thick indium tin oxide (ITO) layer, as well as Pt-contacts were sputtered to create an integrated heater. In the same glass substrate, but on its other side, micropits of $2-\mu \mathrm{m}$ in diameter and with an 1:1 aspect ratio were dry-etched, after which fluorocarbon was deposited to render the micropits hydrophobic. A bubble departure radius of $223 \mu \mathrm{m}$ was predicted for a $1-\mu \mathrm{m}$ radius micropit using Equation 5.1, with $1.324 \mathrm{~kg} \cdot \mathrm{m}^{-3}$ and $998.20 \mathrm{~kg} . \mathrm{m}^{-3}$ the density of air and water at $293 \mathrm{~K},{ }^{23-24}$ respectively, and $7.28 \mathrm{e}^{-2} \mathrm{~N} \cdot \mathrm{m}^{-1}$ the surface tension of air/water at $293 \mathrm{~K},{ }^{25}$ and $9.81 \mathrm{~m} . \mathrm{s}^{-2}$ the gravitational constant. This bubble size is smaller than the hydraulic diameter of the channel $(512 \mu \mathrm{m})$, so that bubbles should not block fluid flow. 
Silicon wafer processing

The microfluidic device was manufactured by standard microfabrication techniques using commercially available silicon $(100 \mathrm{~mm}$ diameter, <100>, p/boron-doped, $525 \mu \mathrm{m}$ thick, Okmetic). Firstly, a 500-nm thick $\mathrm{SiO}_{2}$ layer was grown (thermal wet oxidation at $1423 \mathrm{~K}$ ), followed by photolithography to transfer the fluidic channel design onto the wafer. The pattern was then etched into the silicon oxide layer by reactive ion etching (Adixen AMS-100). After removing the photoresist, the channels were etched into the silicon by deep reactive ion etching (SPTS Pegasus, Bosch type process with a high rate etch process at $273 \mathrm{~K}$ ) using the patterned silicon oxide layer as a hard mask. Since the channels were etched completely though the wafer, an unprocessed silicon wafer was attached to the backside of the processed wafer to avoid leakage of the backside cooling gas during etching. Finally, the silicon wafer was cleaned $\left(\mathrm{O}_{2}\right.$ plasma, 60 min) and the remaining oxide layer stripped in hydrofluoric acid (50\% HF solution).

\section{Glass wafer processing}

Glass wafers (MEMPax, $100 \mathrm{~mm}$ diameter, $500 \mu \mathrm{m}$ thick, Schott AG) were used for the bottom and top layers of the microfluidic device. A photolithography step using a mask containing the micropit pattern was performed on the bottom glass wafer (indicated as 2. in Figure 5.1), followed by continuous reactive ion etching (Adixen AMS 100, 10min, $21 \mathrm{sccm} \mathrm{C}_{4} \mathrm{~F}_{8}, 200 \mathrm{sccm} \mathrm{He}, 10$ sccm Ar, $12 \mathrm{sccm} \mathrm{CH}_{4}$, ICP $2800 \mathrm{~W}$, CCP 350W, Valve: 15\%) to fabricate the micropits. Subsequently, a fluorocarbon film was deposited by plasma enhanced chemical vapor deposition, using an in-house built reactive ion etching machine ("TEtske", $293 \mathrm{~K} ; 11 \mathrm{~W} ; 25$ sccm CHF flow; 130 mTorr; $5 \mathrm{~min}$ ). Following the deposition, the photoresist and the excess fluorocarbon on top of the photoresist were lifted off in an ultrasonic bath (15 min) containing acetone. To produce the fluidic in- and outlets, the other side of the glass wafer was laminated with Harke i-HE dry film photoresist and exposed to a mask featuring the in- and outlet design. After development in water, the glass wafer side with the micropits was protected with an adhesive foil (Nitto SWT 10) and the in- and outlets were powder-blasted into the glass (in-house built micro-powder blaster; 25$\mu \mathrm{m}$ diameter $\mathrm{Al}_{2} \mathrm{O}_{3}$ particles; 5 bar). Afterwards, the foils on both sides were removed. 
Stack assembly and post-processing

After cleaning the silicon wafer in Piranha $\left(\mathrm{H}_{2} \mathrm{SO}_{4}: \mathrm{H}_{2} \mathrm{O}_{2}, 3: 1 \mathrm{vol} / \mathrm{vol}\right)$ and the glass wafer in acetone and isopropanol, the silicon and processed glass wafers were aligned and anodically bonded (EVG EV-501; $673 \mathrm{~K} ; 15 \mathrm{~min} ; 1000 \mathrm{~V}$ ). A second, unprocessed, glass wafer (indicated with 1. in Figure 5.1 B.) was anodically bonded to the other side of the silicon wafer to create the 3-layered stack.

The stack was diced into separate devices, which were next individually coated with ITO (in-house built reactive sputter tool "TCOater") followed by an annealing step to enhance the ITO conductivity (30 min; $673 \mathrm{~K} ; \mathrm{N}_{2}$ atmosphere). Finally, a 100-nm thick platinum layer (in-house sputter tool "TCOathy"; 10 min; $200 \mathrm{~W}$ ) was sputtered on a 10-nm thin tantalum adhesion layer ( 1 min; 200W) on the devices using a Kapton foil shadow mask to create the contact pads for the heater.

\subsubsection{Microfluidic set-up}

The microfluidic device was placed into a dedicated home-made polyether ether ketone (PEEK) chip holder containing an optical window, allowing microscopic imaging of the fluidic channel (Figure 5.2). A power supply was connected to pushpin connections in the top plate of the chip holder, which touched the Ptcontacts on the microfluidic device. A K-type thermocouple was taped to the glass top layer (non-coated glass layer) using yellow Kapton tape to monitor the temperature during the experiments.

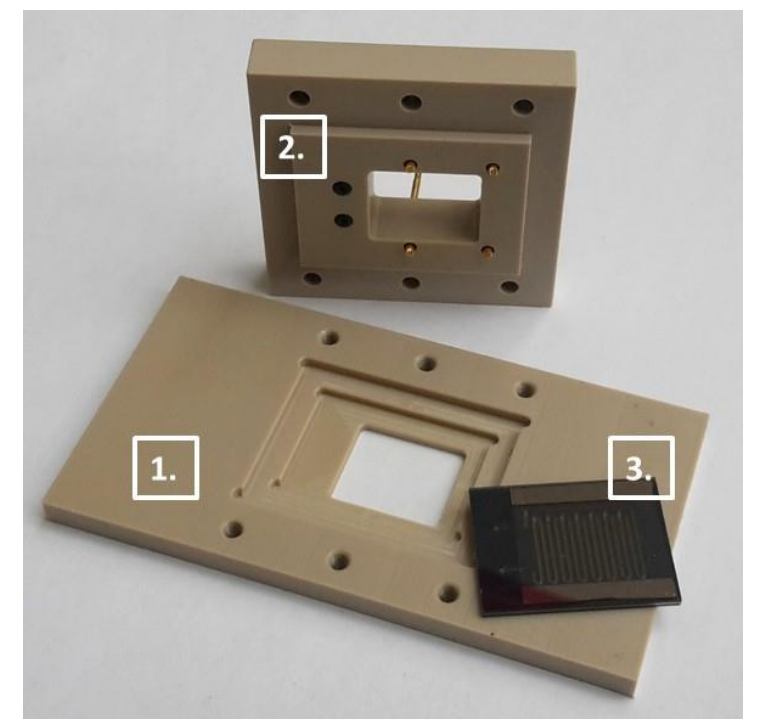

Figure 5.2 PEEK chip holder and microfluidic device with the bottom plate with an optical window (1.), the top plate, also with an optical window, and fluidic connections and pushpins (2.), and the device (3.).

\subsubsection{Bubble formation and confocal microscopy imaging}

As the microfluidic device did not contain any catalyst, bubbles were generated either through boiling of pure MilliQ water or $\mathrm{CO}_{2}$ outgassing from household sparkling water. In both cases, the 
device was heated at atmospheric pressure until gas formation was observed, and operated either with a flow rate of $10 \mu \mathrm{L} \cdot \mathrm{min}^{-1}$ or without any flow.

For confocal microscopy imaging, bubbles were formed using $\mathrm{CO}_{2}$ outgassing, followed by infusing a $0.001 \mathrm{wt} \%$ Fluoroscein isothiocyanate dextran (FITC dextran, average molecular weight 50,000 ) solution in MilliQ water at a flow rate of $10 \mu \mathrm{L} \cdot \mathrm{min}^{-1}$. Images were recorded using an inverted Nikon A1 confocal microscope.

\subsubsection{D-COMSOL Multiphysics model}

\section{Geometry}

A 3D-model was considered to study the momentum, concentration gradient and the conversion of a model reaction at steady-state in the microreactor described above using a finite element model in COMSOL Multiphysics $5.3 \mathrm{a} .{ }^{26}$ Specifically, the simulations aimed at qualitatively studying the effect of bubbles on the convection and the directionality of the liquid flow. To study the concentration gradient resulting from an arbitrary model reaction, a solution containing an arbitrary substrate $\mathrm{C}$ was considered at an initial concentration of $1 \mathrm{~mol} . \mathrm{m}^{-3}$ at the inlet of the microreactor.

A small channel section of $2 \mathrm{~mm}$ in length, $500-\mu \mathrm{m}$ in width and $525-\mu \mathrm{m}$ in height was considered. Two spherical bubbles were considered pinned at the silicon side wall with a radius of a $180-\mu \mathrm{m}$

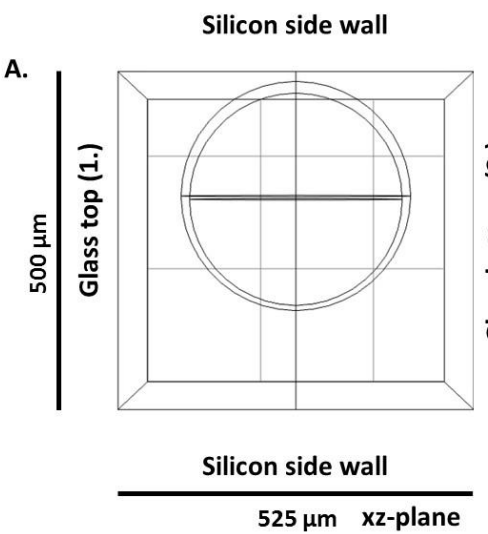

B.

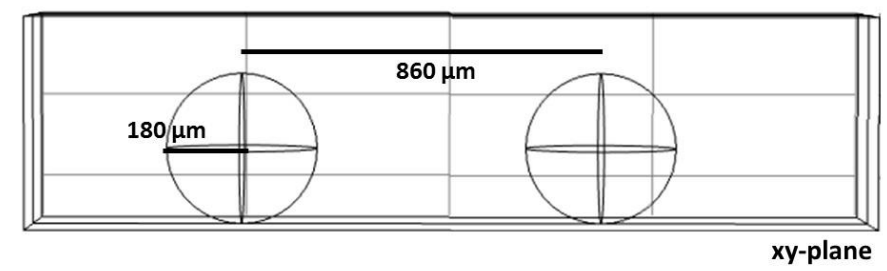

C.

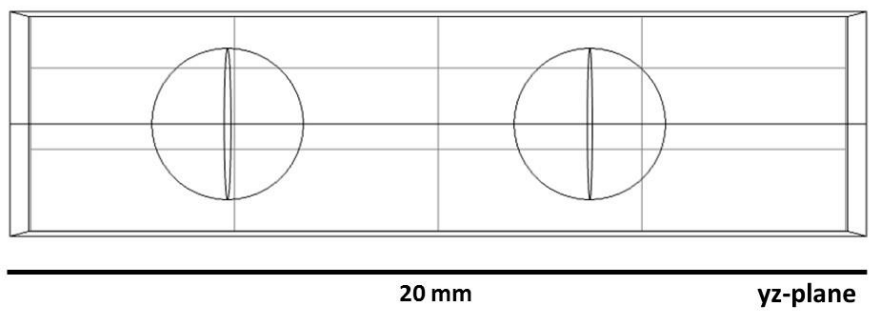

Figure 5.3 Geometry considered in the 3D-COMSOL simulations from 3 different perspectives, xz-plane (A.), xy-plane (B.) and yz-plane (C.) 
and spaced by $860-\mu \mathrm{m}$. The location of the catalytic surface on which a model reaction took place was varied with respect to the wall on which the bubbles were pinned.

The microchannel was treated as a no-slip interface, since no surfactant was added to the solution, with the exception of the no-shear bubble/liquid interface, to which a slip-boundary condition was applied. ${ }^{15-16}$ An entrance length of $1 \mathrm{~m}$ was considered to ensure a fully developed velocity profile and a constant pressure condition was applied to the outlet. The volumetric flow rate was set at 1 $\mu \mathrm{L} . \mathrm{min}^{-1}$. The substrate C concentration of $1 \mathrm{~mol} . \mathrm{m}^{-3}$ was fixed at the microchannel inlet, which is sufficiently diluted to apply Fick's law. An arbitrary first-order reaction with a kinetic constant of $0.001 \mathrm{~s}^{-1}$ was considered at the catalytic surface, whereas a no flux boundary condition was applied to all other interfaces. The Damköhler number, which is the ratio between the reaction time scale and the diffusion mass transfer time scale, was 525 in this particular case, indicating that the microreactor operated in the diffusion-limited regime. Therefore, kinetic limitations of the concentration gradient were not present in this particular system, so that any change in substrate

conversion would only be caused by changes in the mass transport. A $2^{\text {nd }}$ order Lagrange polynomial was used to calculate the concentration profile and for the velocity/pressure was constrained according to a P2-P1 discretization basis.

\section{Governing equations}

The momentum was evaluated by solving the Navier-Stokes equation for compressible fluids and the continuity equation:

$$
\begin{aligned}
& \rho(\vec{u} \cdot \nabla) \vec{u}=-\nabla P+\nabla \cdot \mu\left[(\nabla \vec{u})+(\nabla \vec{u})^{T}\right] \\
& \nabla \cdot(\rho \vec{u})=0
\end{aligned}
$$

with $\rho$ being the density and $\mu$ the viscosity of the liquid phase, $\vec{u}$ the average mean velocity, $P$ the pressure.

The bulk concentration in the microchannel was studied by solving the convection-diffusion equation:

$$
\nabla \cdot\left(-D_{c} \nabla c+\vec{u} c\right)=0
$$

with $D_{c}$, the diffusivity constant, arbitrarily set to $1 \mathrm{e}^{-9} \mathrm{~m}^{2} \cdot \mathrm{s}^{-1}$. 


\subsection{Results and discussion}

\subsubsection{Heater performance, micropit activity and device characterization}

The performances of the integrated ITO-based heater and micropits were first evaluated individually. A single glass layer containing micropits only (no microchannel) was placed in a tank with supersaturated $\mathrm{CO}_{2}$ solution, and outgassing was induced by reducing the pressure in the tank. The microbubbles formed along the micropit pattern and were regularly spaced apart (Figures 5.3A.and B.). These images confirmed that the microcavities were active nucleation spots. Thermal imaging using an IR camera (FLIR ONE Gen 3 Pro - IOS, resolution $0.1 \mathrm{~K}$ ) revealed uniform heating of the microfluidic device (Figure 5.3C.) by the integrated ITO heater.

After assembling the 3-layered stack, the microfluidic devices were calibrated for current, power and, most importantly, temperature as a function of the applied voltage. An external power supply was connected to the pushpin connections touching the Pt-contacts of the device and the voltage was increased in steps of 1 or $2 \mathrm{~V}$, starting from $5 \mathrm{~V}$. From the graphs for current and power in Figure 5.4, an estimated resistance of 60-85 Ohm was determined. These graphs also demonstrate that the electric properties varied between the multiple devices, likely due to the fabrication process, suggesting, for example, a non-uniform ITO layer. As ITO was sputtered device by device, variations in the ITO layer could have occurred between the samples. Therefore, every individual device must be calibrated before use.

A.

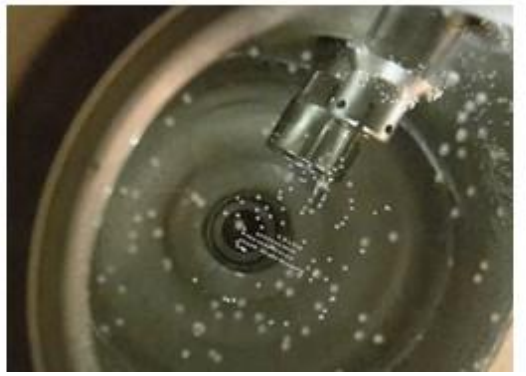

B.

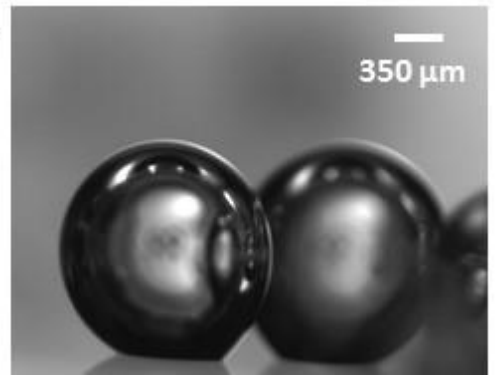

c.

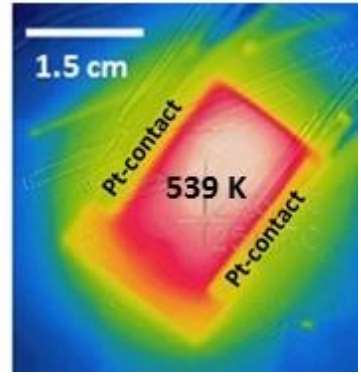

Figure 5.3 Bubble nucleation in a supersaturated $\mathrm{CO}_{2}$ /water mixture on a glass substrate containing only the hydrophobic pits, observed from the top (A) and side (B). The bubbles were generated along the meandering pattern formed by the hydrophobic pits etched in the glass substrate. C. ITO heater, thermal image (IR camera), with red/white indicating a temperature of $539 \mathrm{~K}$, whereas the surroundings in blue were at room temperature. This image indicated a homogeneous temperature field between the electrodes. 

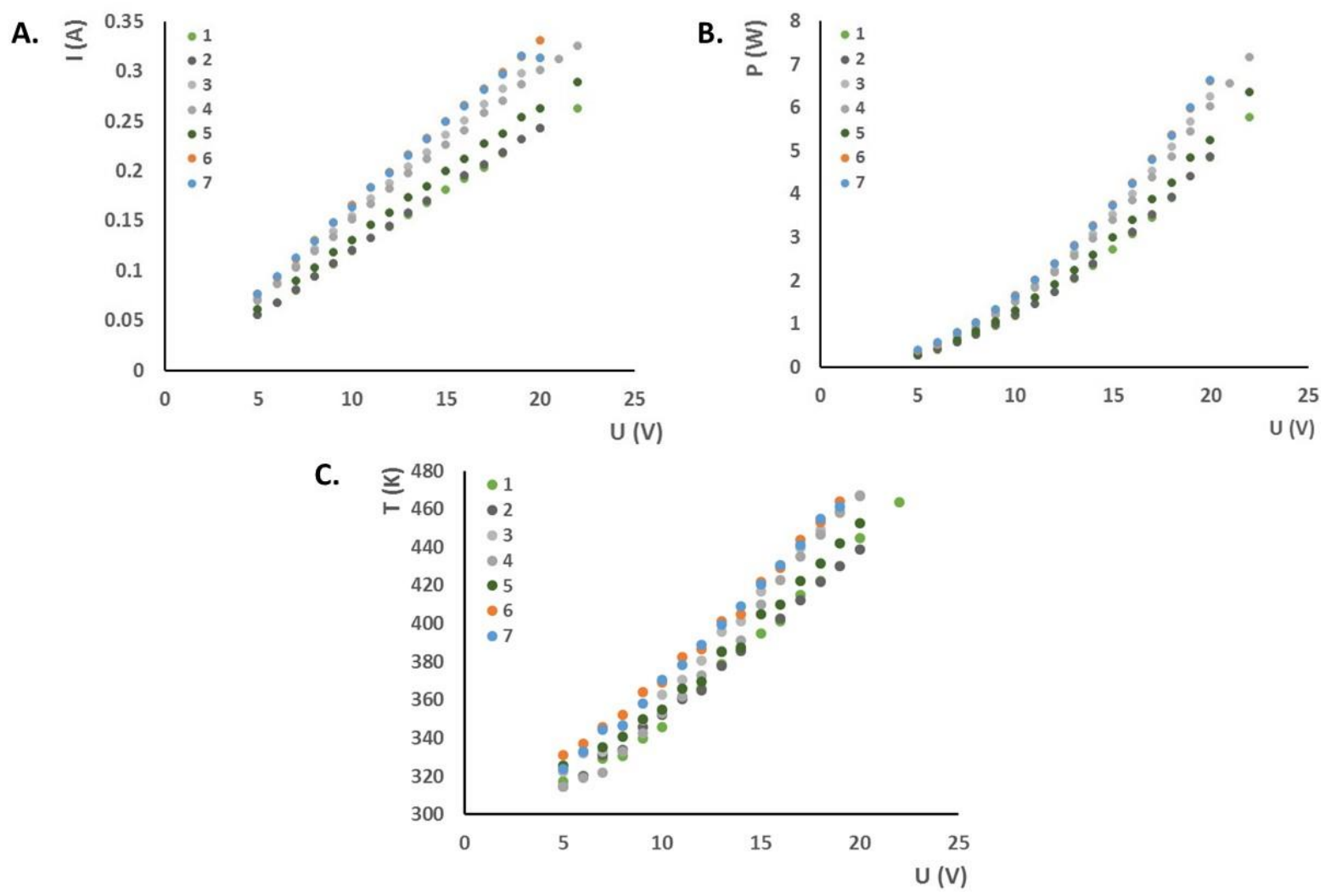

Figure 5.4 Calibration of the integrated ITO-heater: Recorded current (A.), power (B.) and temperature (C.) as a function of the applied voltage to the Pt-contacts on 7 different microfluidic devices placed in the chip holder.

\subsubsection{Controlling bubble formation}

Initially, vapor bubbles were created in closed microchannels by boiling pure MilliQ water, and two devices were compared, with and without micropits. In the reference design without micropits, explosive boiling was observed at $388 \mathrm{~K}$, where a gas phase suddenly forms in the liquid phase (Figure 5.5A.). These results are in good agreement with those reported in Chapter 3. As discussed in that chapter, the additional $15 \mathrm{~K}$ is indicative of superheating, which is consistent with the observed boiling mechanism. In devices with micropits, however, the first vapor bubbles already formed at $358 \mathrm{~K}$ (Figure 5.5B.). These results indicate that the presence of micropits reduced the energy barrier for bubble nucleation, which is consistent with nucleate boiling.

However, during boiling, bubbles rapidly formed in the liquid phase and therefore the bubble size was difficult to control. Formation of gas bubbles out of a supersaturated $\mathrm{CO}_{2} / \mathrm{H}_{2} \mathrm{O}$ solution by 
A.

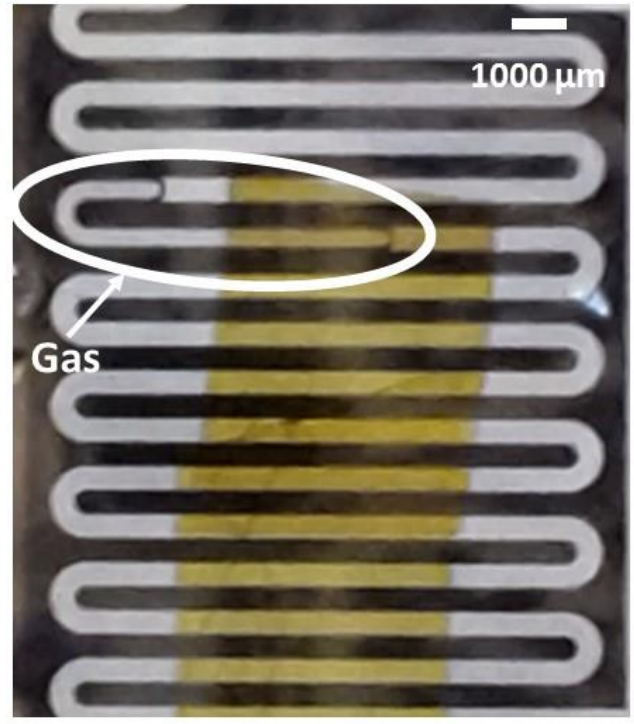

B.

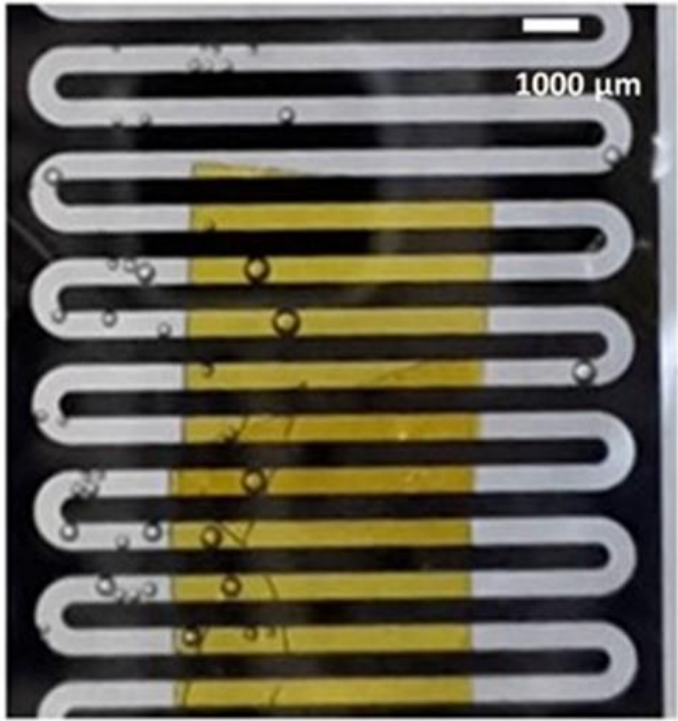

Figure 5.5 Vapor bubbles forming in a microchannel without (A.) and with micropits (B.). Without micropits, explosive boiling was observed at $388 \mathrm{~K}$, where a large gas plug formed in the liquid phase. With micropits, however, nucleate boiling was achieved at $358 \mathrm{~K}$, where multiple small bubbles formed in the liquid phase. Kapton tape, in yellow, was used to hold the thermocouple in place.

slowly increasing the temperature at constant ambient pressure was a more gentle approach; the $\mathrm{CO}_{2}$ solubility decreases when increasing the temperature, resulting in outgassing, bubble nucleation and bubble growth. Using this approach, the first bubbles were observed at a temperature as low as $318 \mathrm{~K}$, which continued growing as long as heat was applied to the device (See also Figure 5.7).

To study possible FITC dextran concentration gradients at the bubble/interface and at the catalytic surface, however, a steady-state regime is desired, i.e. with static, non-growing or departing bubbles. To obtain such a scenario, the microchannel was first completely filled with a $\mathrm{CO}_{2} / \mathrm{H}_{2} \mathrm{O}$ solution perfused at $10 \mu \mathrm{L} \cdot \mathrm{min}^{-1}$. Next, outgassing was induced by shortly heating the device to 318-323 K as before, without liquid flow. By switching off the heat right after the first bubbles were observed, the bubbles could be kept smaller than the channel hydraulic diameter $(512 \mu \mathrm{m})$. Therefore, the liquid could still flow freely pass the gas bubbles, preventing local pressure buildup. 


\subsubsection{Bubble stability and micropit deactivation}

The microbubble pinning strength in the microchannel was qualitatively characterized by gradually increasing the liquid flow rate. Surprisingly, the bubbles remained fixed at their location in the microchannel at least up to a flow rate of $250 \mu \mathrm{L} \cdot \mathrm{min}^{-1}$, indicating strong pinning. The bubbles could be removed, however, by large gas slugs, which formed when a bubble was growing to a size larger than the microchannel hydraulic diameter (bubble length larger than $512 \mu \mathrm{m})$. Furthermore, these results suggest that bubbles were pinned beyond the micropit radius, and therefore had a larger departure radius than was calculated. To prevent large bubbles from forming, slightly larger microchannel can be designed, while keeping the micropit radius the same. Larger slugs also

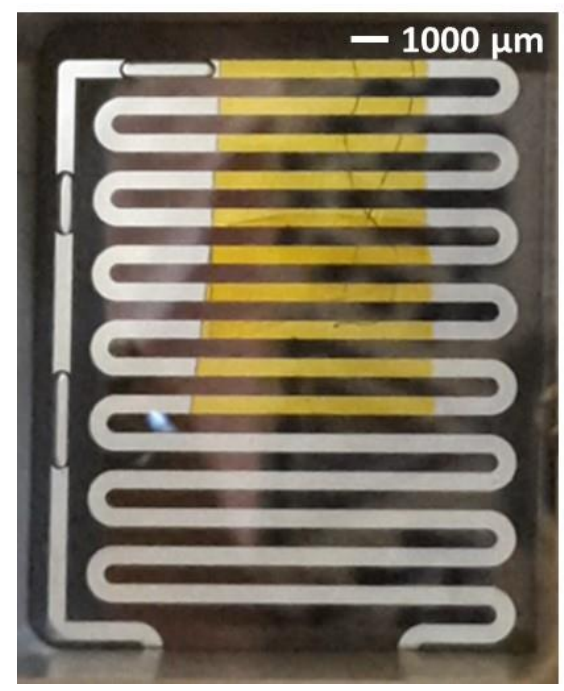

Figure 5.6 Outgassing occurring at the channel inlet: Large $\mathrm{CO}_{2}$ bubbles already forming at the inlet of the device (bottom left of the figure).

often formed at the inlet of the device (Figure 5.6), indicating that outgassing occurred as soon as the cold liquid came into contact with the heated device.

Repeating the nucleation experiments revealed that micropit deactivation occurred after about 20 cycles of nucleation (Figure 5.7). The exact deactivation mechanism is unclear, although two different phenomena could contribute to the deactivation: filling of the micropits with water, or removal of the hydrophobic fluorocarbon layer in the micropits. Water could fill the micropits either due to a too high hydrodynamic pressure, or through formation of a small microjet after a bubble has collapsed. ${ }^{27}$ Furthermore, the force associated with the microjet could remove the fluorocarbon layer. Altogether, once the gas, which was trapped in the micropit, was removed, no new bubbles could grow from that particular cavity.

However, it was possible to reactivate the micropits by first clearing the microfluidic device of the liquid and subsequently heating it at $448 \mathrm{~K}$ for 45 minutes. Next, the microchannel was carefully refilled with liquid at a flow rate of $10 \mu \mathrm{L} \cdot \mathrm{min}^{-1}$, to ensure the hydrodynamic pressure was low enough so that the liquid was not forced in the micropits. Nucleation experiments were then resumed, showing that the micropits could temporarily be re-activated for around 5 cycles (Figure 5.7E). 


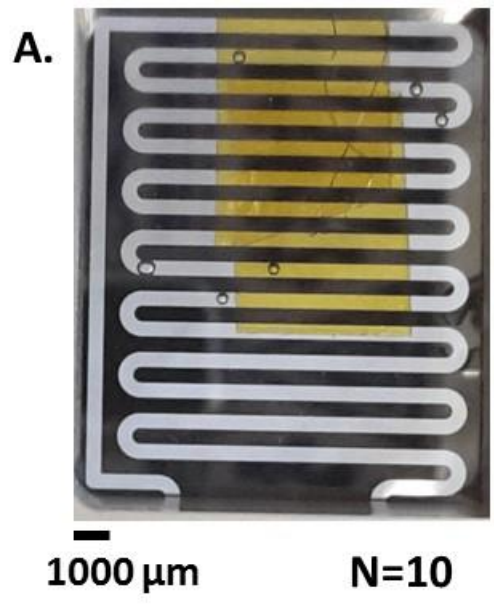

B.

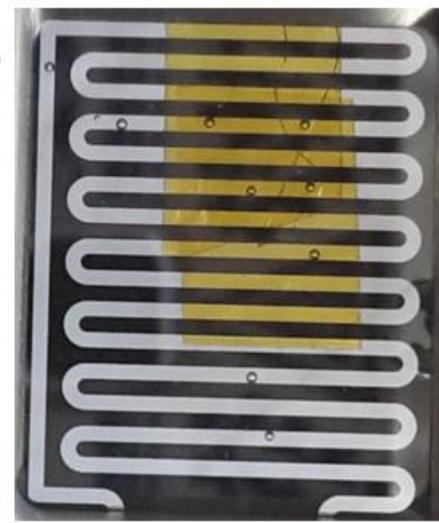

$\mathbf{N}=17$
C.

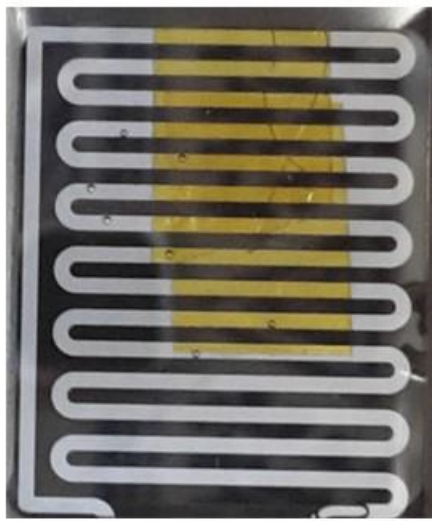

$\mathbf{N}=19$
D.

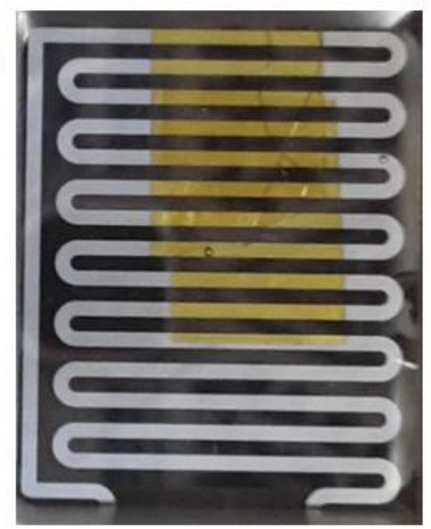

$\mathbf{N}=\mathbf{2 0}$
E.

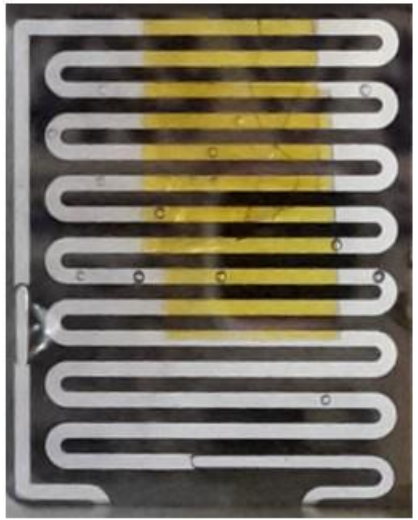

After reactivation

Figure 5.7 Micropit deactivation and reactivation, with $\mathrm{N}$ being the number of nucleation cycles. After about 20 cycles (D), almost no nucleation was observed. The micropits were successfully reactivated by heating for 45 minutes at $448 \mathrm{~K}(\mathrm{E})$.

\subsubsection{Bubble distribution in the microchannel}

Bubbles typically formed 550 to $1000 \mu \mathrm{m}$ from each other (Figure 5.7), which could be explained by the local pressure increase associated with bubble nucleation: if a bubble would nucleate close to another bubble, it would have to overcome a slightly higher pressure. This hypothesis was further supported by the observation that a bubble in close proximity to another, already growing bubble, nucleated at a later time than the first bubble. 


\subsubsection{Bubble geometry: confocal microscopy}

To investigate the bubble geometry in more detail as well as their specific location in the channel, confocal microscopy imaging was performed. First, bubbles were formed by $\mathrm{CO}_{2}$ outgassing, as described previously. Next, the device in its chip holder was placed onto the confocal microscope stage and the fluidic channel was infused with a $0.001 \mathrm{wt} \%$ FITC dextran solution at a flow rate of $10 \mu \mathrm{L} \cdot \mathrm{min}^{-1}$.

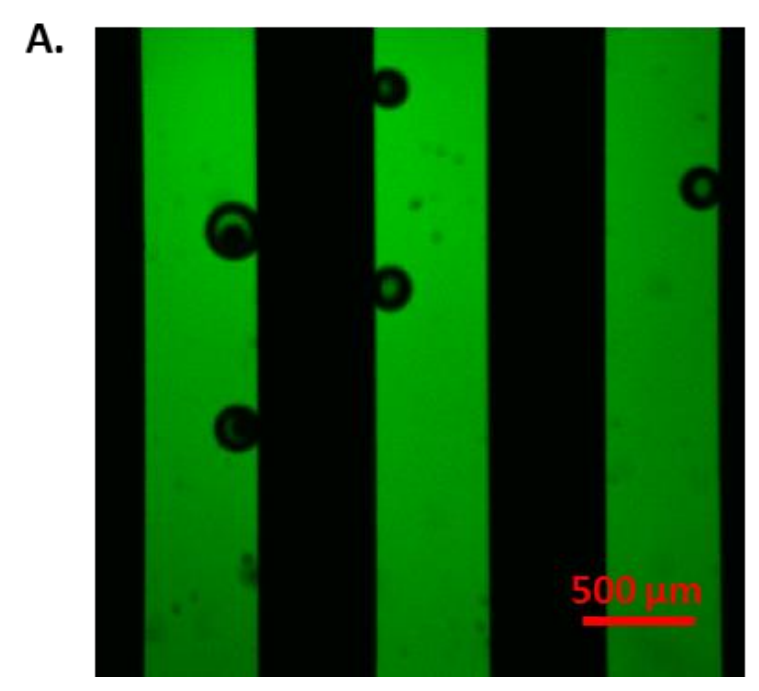

B.

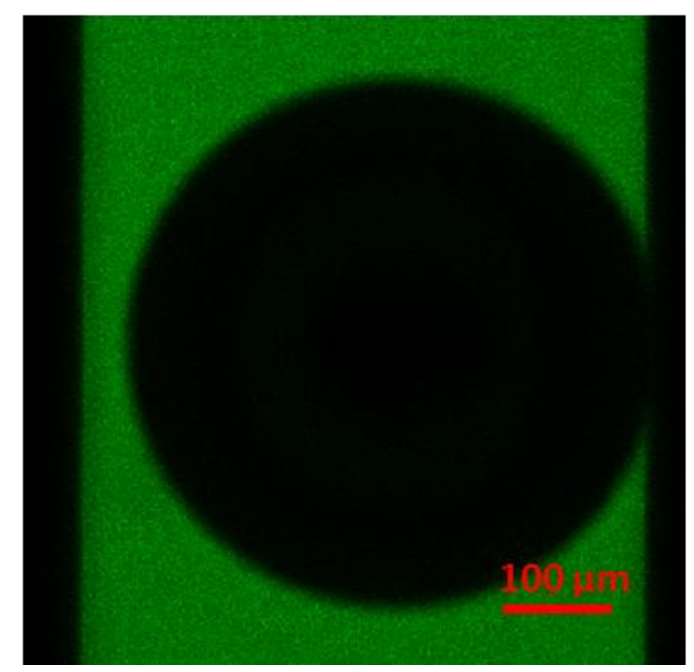

Figure 5.8 Confocal microscopy images of the microfluidic channel showing multiple bubbles (A.) or a single bubble (B.) when infusing FITC dextran at a flow rate of $10 \mu \mathrm{L} \cdot \mathrm{min}^{-1}$ (bottom view).

Figure 5.8A. presents a section of the microfluidic channel with several bubbles. It was observed that bubbles systematically nucleated from the side of the channel instead of from the bottom, as was expected, despite the presence of hydrophobic micropits. As discussed in Chapter 3, dryetching of silicon gives rise to defects on the silicon walls, or so-called scallops, which can also be hydrophobic due to remaining fluorocarbon residues. Examination of both the micropits and the microchannel wall using high-resolution SEM demonstrated that the micropits were very similar in size as the etching defects, around $2 \mu \mathrm{m}$, Figure 5.9. Overall, both the intentional micropits and the scallops can equally act as nucleation sites for the bubbles.

A single bubble was further studied to detect if any FITC dextran concentration gradients would occur on the bubble interface and near the pinning area of the bubble to the microchannel wall. No FITC dextran concentration gradient could be observed in this channel without a catalytic reaction. 

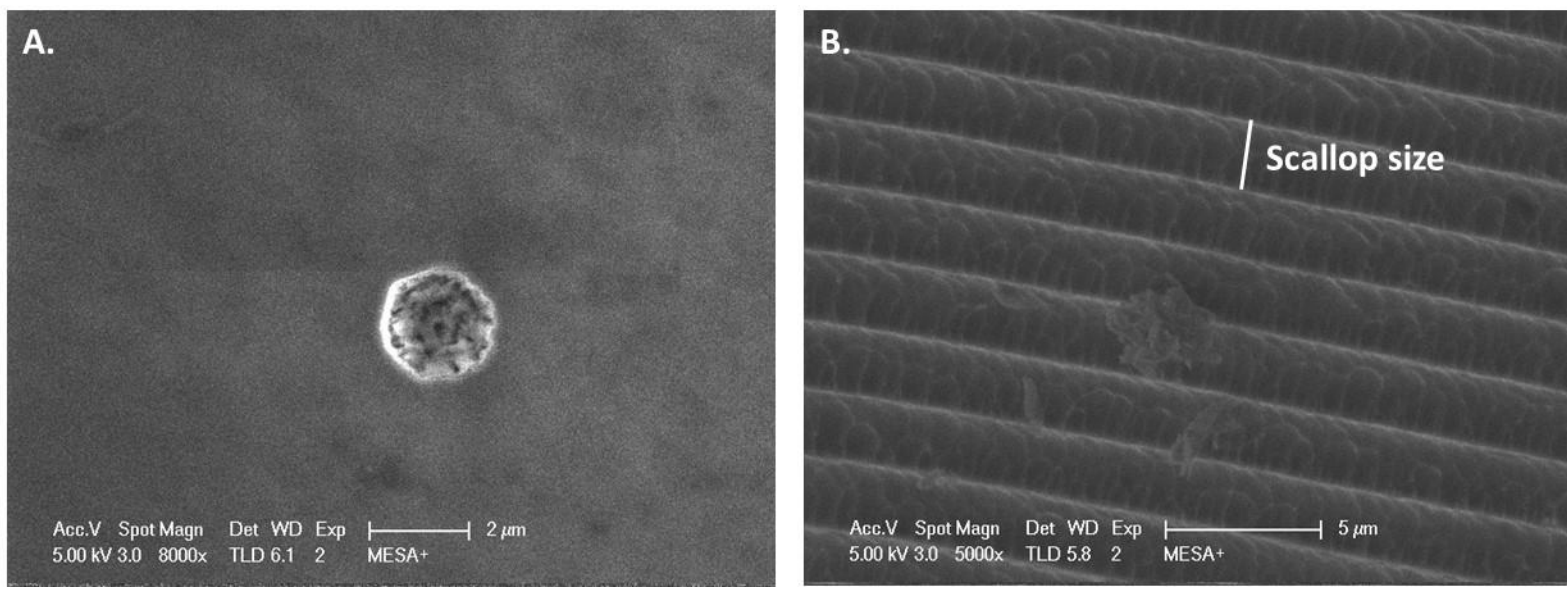

Figure 5.9 SEM images of a hydrophobic micropit etched in glass (A.) and etching scallops (B.). Both structures were roughly $2-\mu \mathrm{m}$ in size.

\subsubsection{Numerical investigation of the momentum and transport}

To determine if there was a FITC dextran gradient present, a 3D-model was developed in COMSOL Multiphysics 5.3a. Figure 5.10 depicts the fluid and concentration profiles of an arbitrary selected substrate $\mathrm{C}$ for a $500-\mu \mathrm{m}$ by $525-\mu \mathrm{m}$ microchannel with two $180-\mu \mathrm{m}$ radius bubbles in absence of a catalytic layer. The fluid velocity reached its maximum value of $20.10^{-5}$ $\mathrm{m} \cdot \mathrm{s}^{-1}$ at the top of the bubbles.

Negligible concentration differences of 0.99 to $1.01 \mathrm{~mol} . \mathrm{m}^{-3}$ were found, which is in accordance with the experimental data in Figure 5.8. Tailing was observed after the bubbles, which has also been reported in the literature for other objects obstructing fluid flow. ${ }^{28}$

\subsubsection{Influence of pinning location of spherical bubbles on the catalytic surface}

Next, a catalytic layer was incorporated in the model to evaluate the effect of bubbles on the conversion for a first-order catalytic reaction. In the presence of a catalytic layer, a larger concentration gradient was expected as a result of the chemical reaction. The conversion was evaluated in four configurations, (i) with no bubbles, and with bubbles and a catalytic layer (ii) on the same wall as on which the bubbles were pinned, (iii) on the opposite wall to that with the bubbles, or (iv) on the side wall with respect to the pinned bubble. 
A. Velocity $\left[\mathrm{m} \cdot \mathrm{s}^{-1}\right]$

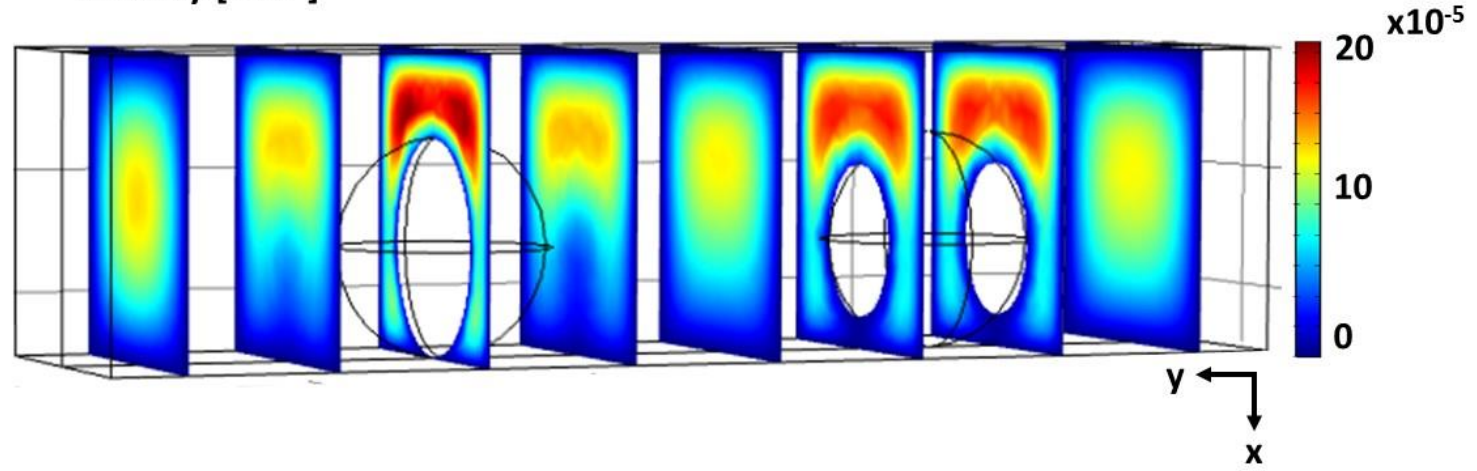

B. Concentration $\left[\mathrm{mol} . \mathrm{m}^{3}\right]$

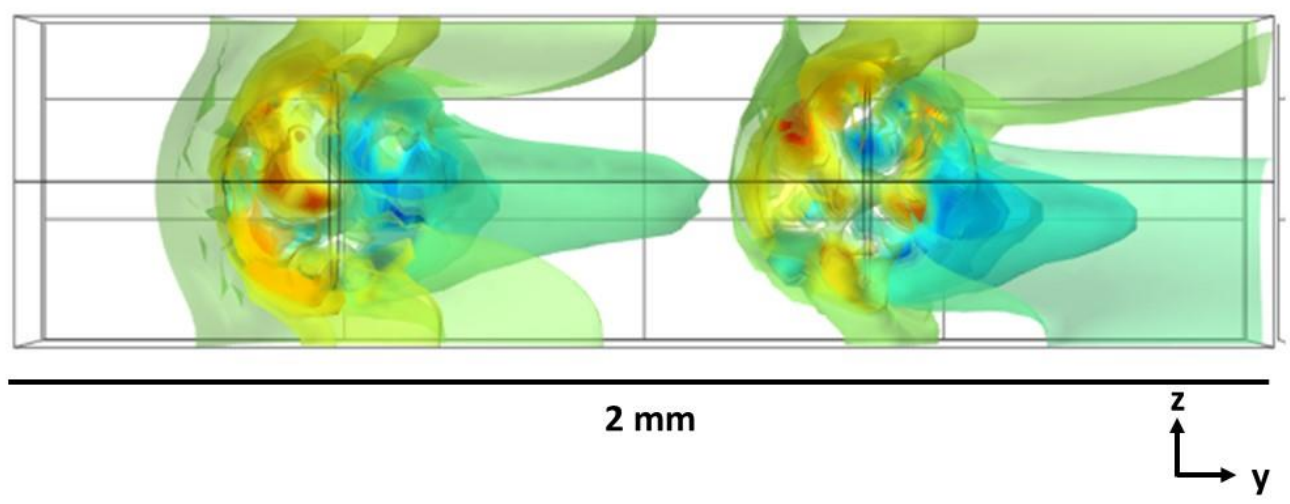

$\mathbf{x} 10^{-3}$

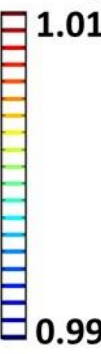

Figure 5.10 Velocity (A., perspective from the side) and concentration (B., bottom view) profile in a microchannel with two $180-\mu \mathrm{m}$ radius bubbles without a catalytic layer. The velocity is at the highest at the top of the bubbles. A negligible concentration difference of $0.02 \mathrm{~mol} . \mathrm{m}^{-3}$ was found, which is in accordance with the experimental data in Figure 5.8.

In all configurations, the substrate conversion was determined by dividing the outlet concentration by the inlet concentration of substrate $\mathrm{C}$. Table 5.1 summarizes the results and shows that compared to the configuration without bubbles, the conversion increased in all configurations, except for the case where the catalyst was on the same wall as the bubbles.

The difference in conversion between the four configurations can be explained by the directionality of the convective flow, as shown in Figure 5.11, which depicts the velocity arrow plots for the four discussed configurations, with the catalytic wall indicated in red. For the configuration without bubbles (Figure 5.9B.), the velocity, and therefore the convective transport was directed from the inlet to the outlet of the microreactor, without any change in the fluid direction. When catalyst was on the side wall with respect to the bubbles (Figure 5.9C.), the fluid was forced around the bubble 
Table 5.1 Conversion of substrate $\mathrm{C}$ in a model catalytic reaction for four different configurations of the catalytic layer with respect to the wall on which bubbles were pinned.

\begin{tabular}{lc}
\hline Geometry & Conversion \\
\hline No bubbles & 0.355 \\
Catalyst on the same wall as the bubbles & 0.326 \\
Catalyst on the side wall with respect to the bubbles & 0.382 \\
Catalyst on the opposite wall from the bubbles & 0.444 \\
\hline
\end{tabular}

towards the catalyst. The increase in velocity towards the catalyst resulted in a slight increase in conversion, from 0.355 to 0.382 .

However, when the catalytic layer was on the same wall on which bubbles were pinned, the conversion decreased to 0.326 . In this configuration, the fluid was pushed towards the small area between the top of the bubble and the top channel wall, which is opposite to the catalyst, resulting in a lower conversion. As the fluid was directed towards the wall opposite to the one on which the bubbles were pinned, locating the catalytic layer on that wall resulted in a higher conversion of 0.444 .

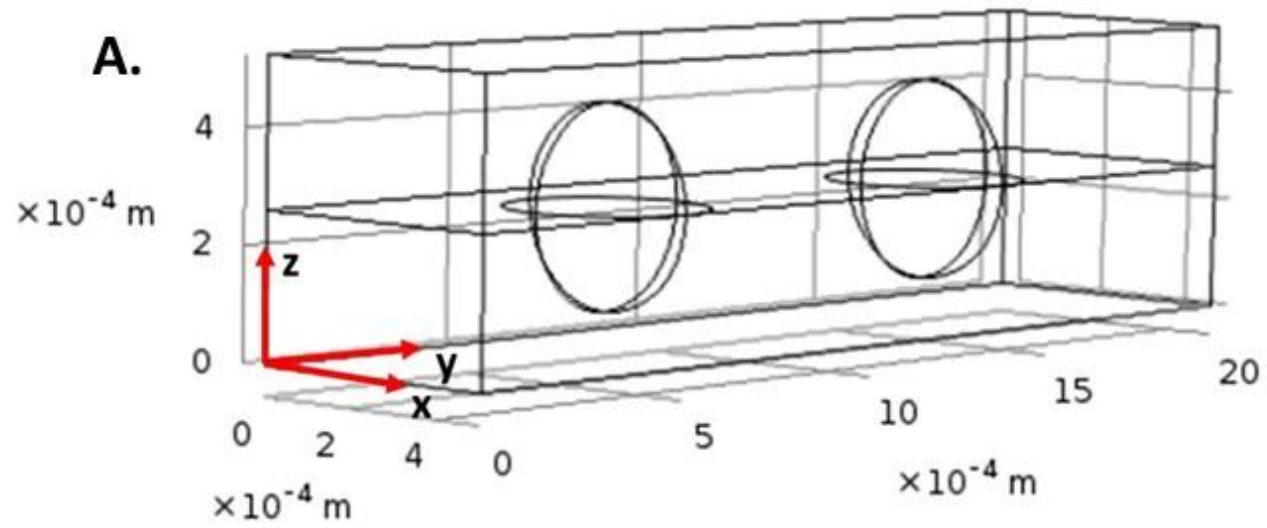

B.

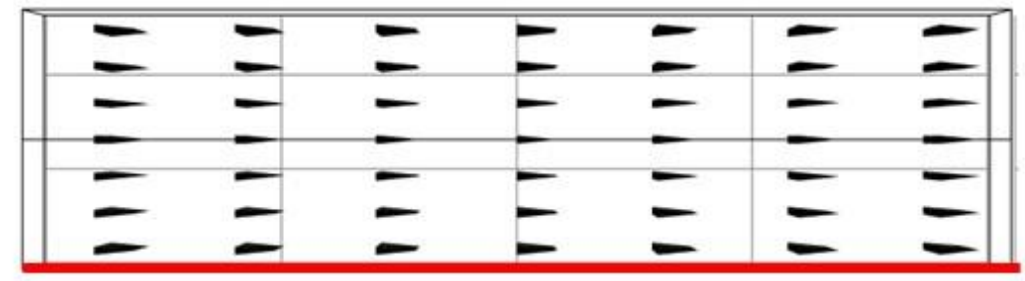

No bubbles, zy-plane 
C.

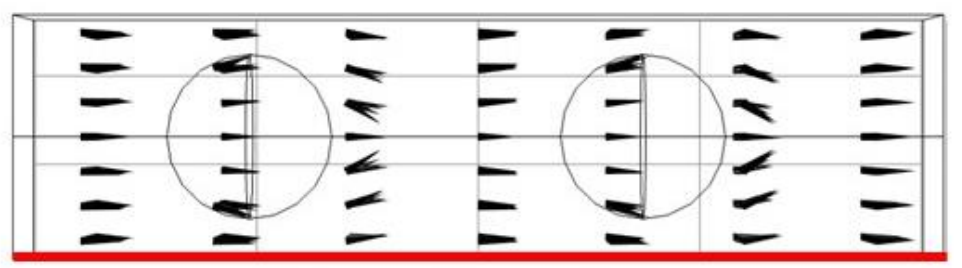

Side wall, yz-plane

D.

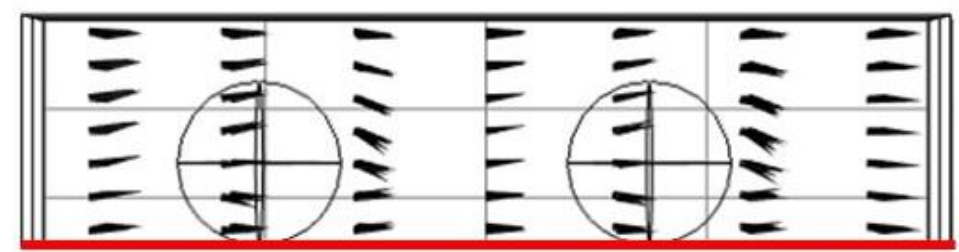

Pinning wall, $x y$-plane

\section{E.}

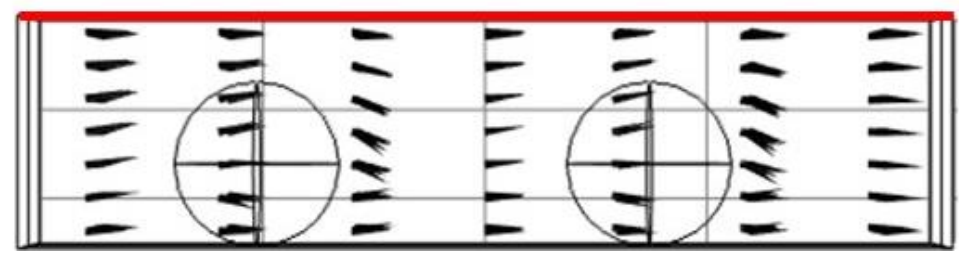

Figure 5.11 Direction of the velocity in the considered microreactor section without bubbles (B.) or with two bubbles (C., D., and E.). The location of the catalytic wall, indicated in red, was varied between the three geometries with respect to the wall on which the bubbles were pinned.

\subsection{Conclusion}

Transport phenomena in multiphase microsystems in which gas bubbles grow on a catalytic surface in a liquid phase are complex. In this chapter, a microfluidic device with hydrophobic micropits was fabricated as a possible approach to control bubble nucleation, growth and departure, to ultimately control transport phenomena. By supplying heat to the microsystem pinned bubbles were created in the microchannel either through boiling or $\mathrm{CO}_{2}$ outgassing, which were stable at least up to a flow rate of $250 \mu \mathrm{L} \cdot \mathrm{min}^{-1}$.

Confocal microscopy imaging using FITC dextran revealed that these bubbles nucleated from the etching defects on the channel wall instead of the well-defined micropits. Furthermore, no FTIC dextran gradient was observed near the wall or on the bubble/liquid phase. The absence of a gradient was confirmed by 3D numerical simulations in COMSOL Multiphysics. However, when 
a catalytic layer was introduced on the wall opposite to the one on which the bubbles were pinned in the COMSOL model, the substrate conversion was increased to 0.444 compared to 0.355 in a channel without bubbles. In that configuration, the fluid flow was directed towards the catalytic surface, which resulted in a higher convection near the catalyst and therefore an increase in conversion. Overall, this work demonstrates the importance of controlling the bubble formation in multiphase microreactor to ultimately increase the reactor efficiency.

\section{Acknowledgements}

Many thanks to Dr. Axel Guenther and the members of the Guenther Laboratory, University of Toronto, Canada, where I performed the experimental part of this chapter as a visiting PhD-student for 4.5 months. I would also like to thank Stefan Schlautmann for the microfabrication, Dr. Jeff Wood for his support during the numerical simulations and Dr. Alváro Moreno Soto for his help with the bubble nucleation on the single glass substrate.

\section{References}

1. Cortright, R. D.; Davda, R. R.; Dumesic, J. A., Hydrogen from catalytic reforming of biomass-derived hydrocarbons in liquid water. Nature 2002, 418 (6901), 964-967.

2. Chheda, J. N.; Huber, G. W.; Dumesic, J. A., Liquid-phase catalytic processing of biomassderived oxygenated hydrocarbons to fuels and chemicals. Angewandte Chemie-International Edition 2007, 46 (38), 7164-7183.

3. Davda, R. R.; Shabaker, J. W.; Huber, G. W.; Cortright, R. D.; Dumesic, J. A., A review of catalytic issues and process conditions for renewable hydrogen and alkanes by aqueous-phase reforming of oxygenated hydrocarbons over supported metal catalysts. Applied Catalysis BEnvironmental 2005, 56 (1-2), 171-186.

4. $\quad$ Prakash, M.; Gershenfeld, N., Microfluidic Bubble Logic. Science 2007, 315 (5813), 832835.

5. Meng, D. D.; Kim, J.; Kim, C.-J., A degassing plate with hydrophobic bubble capture and distributed venting for microfluidic devices. Journal of Micromechanics and Microengineering 2006, $16(2), 419-424$.

6. Chung, S. K.; Zhao, Y.; Cho, S. K., On-chip creation and elimination of microbubbles for a micro-object manipulator. Journal of Micromechanics and Microengineering 2008, 18 (9). 
7. Li, J.; Wang, Y. X.; Chen, H. S.; Wan, J. D., Electrowetting-on-dielectrics for manipulation of oil drops and gas bubbles in aqueous-shell compound drops. Lab on a Chip 2014, 14 (22), 43344337.

8. Gogolides, E.; Ellinas, K.; Tserepi, A., Hierarchical micro and nano structured, hydrophilic, superhydrophobic and superoleophobic surfaces incorporated in microfluidics, microarrays and lab on chip microsystems. Microelectron. Eng. 2015, 132, 135-155.

9. Yong, J.; Singh, S. C.; Zhan, Z.; Chen, F.; Guo, C., How To Obtain Six Different Superwettabilities on a Same Microstructured Pattern: Relationship between Various Superwettabilities in Different Solid/Liquid/Gas Systems. Langmuir 2019, 35 (4), 921-927.

10. Jung, W.-B.; Yun, G.-T.; Kim, Y.; Kim, M.; Jung, H.-T., Relationship between Hydrogen Evolution and Wettability for Multiscale Hierarchical Wrinkles. ACS Applied Materials \& Interfaces 2019, 11 (7), 7546-7552.

11. Xu, J. J.; Chen, B. D.; Xie, T. Z. Z., Experimental and theoretical analysis of bubble departure behavior in narrow rectangular channel. Progress in Nuclear Energy 2014, 77, 1-10.

12. Lee, W.; Son, G.; Jeong, J. J., Numerical Analysis of Bubble Growth and Departure from a Microcavity. Numerical Heat Transfer Part B-Fundamentals 2010, 58 (5), 323-342.

13. Ou, J.; Perot, B.; Rothstein, J. P., Laminar drag reduction in microchannels using ultrahydrophobic surfaces. Physics of Fluids 2004, 16 (12), 4635-4643.

14. Bocquet, L.; Lauga, E., A smooth future? Nature Materials 2011, 10, 334.

15. Vinogradova, O. I.; Dubov, A. L., Superhydrophobic textures for microfluidics. Mendeleev Commun. 2012, 22 (5), 229-236.

16. Karatay, E.; Tsai, P. A.; Lammertink, R. G. H., Rate of gas absorption on a slippery bubble mattress. Soft Matter 2013, 9 (46), 11098-11106.

17. Gong, S.; Cheng, P.; Quan, X., Two-dimensional mesoscale simulations of saturated pool boiling from rough surfaces. Part I: Bubble nucleation in a single cavity at low superheats. Int. J. Heat Mass Transfer 2016, 100, 927-937.

18. Jensen, M. J.; Goranovic, G.; Bruus, H., The clogging pressure of bubbles in hydrophilic microchannel contractions. Journal of Micromechanics and Microengineering 2004, 14 (7), 876883.

19. Mohammadi, M.; Sharp, K. V., The Role of Contact Line (Pinning) Forces on Bubble Blockage in Microchannels. J. Fluids Eng. 2015, 137 (3), 0312081-312087. 
20. Oguz, H. N.; Prosperetti, A., DYNAMICS OF BUBBLE-GROWTH AND DETACHMENT FROM A NEEDLE. J. Fluid Mech. 1993, 257, 111-145.

21. Yamamoto, K.; Ogata, S., Control of the bubble departure diameter by saw-tooth surfaces. Colloids and Surfaces a-Physicochemical and Engineering Aspects 2014, 460, 377-385.

22. van der Linde, P.; Penas-Lopez, P.; Soto, A. M.; van der Meer, D.; Lohse, D.; Gardeniers, H.; Rivas, D. F., Gas bubble evolution on microstructured silicon substrates. Energy \& Environmental Science 2018, 11 (12), 3452-3462.

23. Engineering Toolbox Water - Density, Specific Weight and Thermal Expansion Coefficient. https://www.engineeringtoolbox.com/water-density-specific-weight-d_595.html.

24. Engineering Toolbox Air - Density, Specific Weight and Thermal Expansion Coefficient at Varying Temperature and Constant Pressures. https://www.engineeringtoolbox.com/airdensity-specific-weight-d_600.html.

25. Engineering Toolbox Surface Tension of Water in contact with Air. https://www.engineeringtoolbox.com/water-surface-tension-d_597.html.

26. COMSOL AB COMSOL Multiphysics 5.3a, AB, COMSOL: Stockholm, Sweden, 2018.

27. Borkent, B. M.; Gekle, S.; Prosperetti, A.; Lohse, D., Nucleation threshold and deactivation mechanisms of nanoscopic cavitation nuclei. Physics of Fluids 2009, 21 (10).

28. Lee, H.; Gupta, A.; Hatton, T. A.; Doyle, P. S., Creating Isolated Liquid Compartments Using Photopatterned Obstacles in Microfluidics. Physical Review Applied 2017, 7 (4). 
CHAPTER 5 


\title{
Controlled catalyst deposition
}

\begin{abstract}
Several techniques have been reported in the literature to deposit heterogeneous catalysts in microchannels, which can be classified as wet chemical and physical deposition methods. In this chapter, both washcoating, a wet chemical method, and spark ablation, a physical deposition method, were investigated to coat a microchannel with $\mathrm{TiO}_{2}$ and $\mathrm{Cu} / \mathrm{CuO}$, respectively. For washcoating, $\mathrm{a} \mathrm{TiO}_{2}$ sol-gel was perfused through the microchannel to create a thin porous layer at the channel wall. Spark ablation, however, is based on nanoparticle generation from metal electrodes under high voltages, which were then deposited on the substrate with uniform distribution along the microchannel. These two types of layers were characterized by SEM and methylene blue degradation was employed as a model reaction to evaluate the activity of the $\mathrm{Cu} / \mathrm{CuO}$ catalytic layer.
\end{abstract}

Part of this chapter has been adapted from:

Ripken, R.M.; Schlautmann, S., Roels, P., Weber, K., Gardeniers, J.G.E. and Le Gac, S. Controlled catalyst deposition microchannels, In preparation 


\subsection{Introduction}

Aqueous-Phase Reforming (APR) requires a heterogeneous catalyst, which typically consists of a metal active phase on an oxide support. The catalyst should not only be able to perform at typical APR reaction conditions (up to $550 \mathrm{~K}$ and up to $50 \mathrm{bar}$ ), ${ }^{2}$ but also be selective towards hydrogen formation. The catalyst should promote $\mathrm{C}-\mathrm{C}$ cleavage and the water-gas-shift, rather than $\mathrm{C}-\mathrm{O}$ bond scission followed by hydrogenation, which would result in alkane production. ${ }^{3-4} \mathrm{Pt}, \mathrm{Ni}, \mathrm{Ru}$, $\mathrm{Rh}, \mathrm{Pd}$, Ir metals on a $\mathrm{SiO}_{2}$ support, ${ }^{4}$ and $\mathrm{Pt}$ on $\mathrm{ZrO}_{2}, \mathrm{TiO}_{2}, \mathrm{Al}_{2} \mathrm{O}_{3}$ and $\mathrm{ZnO}$ catalyst supports ${ }^{3}$ have been investigated, of which $\mathrm{Pt} / \mathrm{Al}_{2} \mathrm{O}_{3}$, was found to result in good hydrogen selectivity (up to $100 \%)$ and reasonable turnover frequencies (14 per min). ${ }^{3-4}$

When performing APR in a microfluidic reactor, the heterogeneous catalyst can be introduced in different configurations, of which a packed bed ${ }^{6-7}$ is probably the most commonly used. However, this approach suffers from high pressure drops and uncontrolled fluid flows. ${ }^{8}$ Alternatively, a reactor can be filled with a monolith or a catalytic foam, ${ }^{9-10}$ where higher flows can be applied as a result of the porous structure of the catalyst. ${ }^{8}$ However, less catalyst is present compared to the packed-bed geometry and pores easily get clogged, resulting in a loss of catalytic activity. ${ }^{8} \mathrm{~A}$ third possibility is to deposit the catalyst as a thin layer on the microchannel wall. ${ }^{11-12}$ Although the catalyst loading is lower compared to the other two approaches, the other limitations (pressure drop and clogging of the catalyst pores) are circumvented by depositing the catalyst as a thin film. ${ }^{8}$

A wide variety of thin-layer deposition techniques exists today, which can be roughly divided in chemical deposition methods, such as electroplating ${ }^{13}$ and spin coating, ${ }^{14}$ and physical deposition methods, such as sputtering. ${ }^{15}$ These procedures are often executed in a clean room environment, which is not always available, while adding costs.

A relatively easy chemical deposition technique not requiring a clean room to deposit a catalytic thin layer is washcoating, ${ }^{12,16}$ which has also been successfully applied for the catalytic coating of microchannels for APR. ${ }^{12}$ Here, a slurry, or sol-gel consisting of $\mathrm{AlO}(\mathrm{OH})$ particles, a small amount of acid to prevent particle agglomeration and in some cases a binder molecule, is flushed through the microchannel, where it is subsequently deposited. Many factors affect the quality of the final layer: the solid content of the slurry, its viscosity, the residence time of the sol-gel in the channel, the particle size, and the $\mathrm{pH} \cdot{ }^{17-18}$ Furthermore, wet chemical methods often suffer from diffusion limitations, resulting in long production times and inhomogeneous layers. ${ }^{19}$ Still, 
washcoated layers typically have high porosity. ${ }^{14}$ For example, Ambrosetti et al. reported very high pore densities with cell sizes of $580-\mu \mathrm{m}$ in diameter. ${ }^{18}$ Altogether, washcoating is a favorable method to deposit catalytic (support) materials.

In addition to chemical deposition methods, spark discharge generation has been investigated as a physical method to deposit a metal active phase with a uniform distribution over the microchannels. Spark discharge generation continuously produces pure nanoparticles, with a welldefined size and composition at ambient pressure. A high-frequency spark between two electrodes ablates material from the electrode surfaces, and the resulting vapor of atoms is transported through a nozzle using an inert carrier gas. ${ }^{20-21}$ During the residence time from the electrodes to the substrate, the atoms collide and form primary particles (nm-size). The high density of particles in the carrier gas results in agglomeration into larger clusters before deposition. Primary particle size can be controlled by manipulating the spark power or flowrate of the carrier gas (Figure 6.1). ${ }^{20}$ Finally, the nanoparticles are deposited on the substrate by inertial impact.

Compared to other physical deposition techniques, creating nanoparticles by electrical discharge is lower in operational and material costs, which are two major disadvantages of physical deposition methods. ${ }^{20,22}$ In addition, electrical discharge is more flexible in terms of materials, catalyst geometry (for example a core-shell structure), and particle size. In principle, every electrically conducting material can be used to create particles, including alloys.

In this chapter, both washcoating and spark ablation were explored to deposit $\mathrm{TiO}_{2}$ and $\mathrm{Cu} / \mathrm{CuO}$ on silicon substrates. During deposition a masking layer was employed to ensure that the catalyst was solely present in the microchannel. The morphology of the layers was characterized using SEM. The adhesion of the $\mathrm{TiO}_{2}$ layer to the substrate was studied by submersion of the device in boiling water, and the presence of $\mathrm{Cu} / \mathrm{CuO}$ was confirmed by energy-dispersive $\mathrm{X}$-ray (EDX) spectroscopy. Lastly, the activity of the $\mathrm{Cu} / \mathrm{CuO}$ was studied using $\mathrm{UV}$-assisted methylene blue degradation as a model reaction. 


\subsection{Materials and Methods}

\subsubsection{Microfluidic devices}

Microfluidic channels were manufactured in silicon with the same design and procedure as described in Chapter 3. The microfluidic device contained either a 250- $\mu \mathrm{m}$ wide, $250-\mu \mathrm{m}$ deep, $40-\mathrm{cm}$ long channel or a channel of 500- $\mu \mathrm{m}$ in width, $250-\mu \mathrm{m}$ in depth and $20-\mathrm{cm}$ in length. Two wafers were prepared: one was used as received, while on the second wafer a $500-\mathrm{nm}$ thick $\mathrm{SiO}_{2}$ layer was uniformly grown by wet oxidization in an oven at $1423 \mathrm{~K}$ for $26 \mathrm{~min}$.

For washcoating, the channels were covered using UV-cured foil, instead of bonding the processed silicon substrate to a glass wafer. For extra support during the catalyst deposition, the silicon substrate was clamped to either another silicon device or a polydimethylsiloxane (PDMS) slab before placing it into the same chip holder as used in Chapter 3. This PDMS layer was prepared by mixing the monomer (Permacol RTV-615 A) and curing agent (Permacol RTV615-B) in a 10:1 $\mathrm{wt} / \mathrm{wt}$ ratio after degassing in a dessicator. The solution was poured into a petri dish, and degassed again. PDMS was cured at $353 \mathrm{~K}$ for 2 hours in an oven, after which it was removed from the mold and cut to the same size as the silicon device.

For deposition using spark ablation, the channels were left open. To ensure that nanoparticles were only deposited in the microchannel, a photoresist layer was spray coated onto the silicon device. After spray coating, photolithography was performed to remove any photoresist from inside the microchannels. After $\mathrm{Cu} / \mathrm{CuO}$ deposition, the photoresist protective mask was removed by rinsing the device with acetone and isopropanol, followed by sonication in acetone to remove any remaining residues. The processed silicon wafer was anodically bonded (673 K, $1000 \mathrm{~V}, 15 \mathrm{~min})$ to the MEMPax glass top layer. After catalytic testing, a small strip was diced out of the glass top layer, so that the catalytic layer was accesible for SEM characterization.

\subsubsection{Dynamic washcoating}

The crystal phase of aluminium oxide often changes to boehmite under hydrothermal conditions, ${ }^{23-}$ 24 as employed during APR. This change in crystallinity might affect the catalytic activity and, therefore, $\mathrm{TiO}_{2}$ was used here as a model catalyst support instead of the conventionally used $\mathrm{Al}_{2} \mathrm{O}_{3}$. A commercial $30 \mathrm{wt} \% \mathrm{TiO}_{2}$ suspension in water (AERODISP VP DISP W $2730 \mathrm{X}$ ) was diluted in distilled water to obtain a $5 \mathrm{wt} \% \mathrm{TiO}_{2}$ solution. 
The dynamic washcoating procedure was adapted from D'Angelo et al. ${ }^{12}$ First, the fluidic channel was perfused with $1 \mathrm{M} \mathrm{KOH}$ at a flow rate of $30 \mu \mathrm{L} \cdot \mathrm{min}^{-1}$ for 15 minutes to clean the channel from any contaminants, followed by distilled water under the same conditions to remove any remaining $\mathrm{KOH}$. The device containing the $250-\mu \mathrm{m}$ wide channel was filled with the titania suspension, which was then displaced by air using a syringe pump at 10 or $30 \mu \mathrm{L} \cdot \mathrm{min}^{-1}$. Devices with a $500-$ $\mu \mathrm{m}$ wide channel were filled with the same suspension, followed by displacement at $30 \mu \mathrm{L} \cdot \mathrm{min}^{-1}$. The titania layer was calcined at $573 \mathrm{~K}$ for 8 hours, with a heating and cooling rate of $2 \mathrm{~K} \cdot \mathrm{min}^{-1}$. The titania layers were characterized by SEM, before and after exposure to boiling water for 30 minutes.

\subsubsection{Spark ablation}

The catalytic nanomaterial was produced using the VSP-G1 Nanoparticle Generator and immobilized as a nanoporous layer using the Nanostructured Material Printer VSP-P1, both from VSPARTICLE B.V., Delft, The Netherlands. This novel printing technology combines Spark

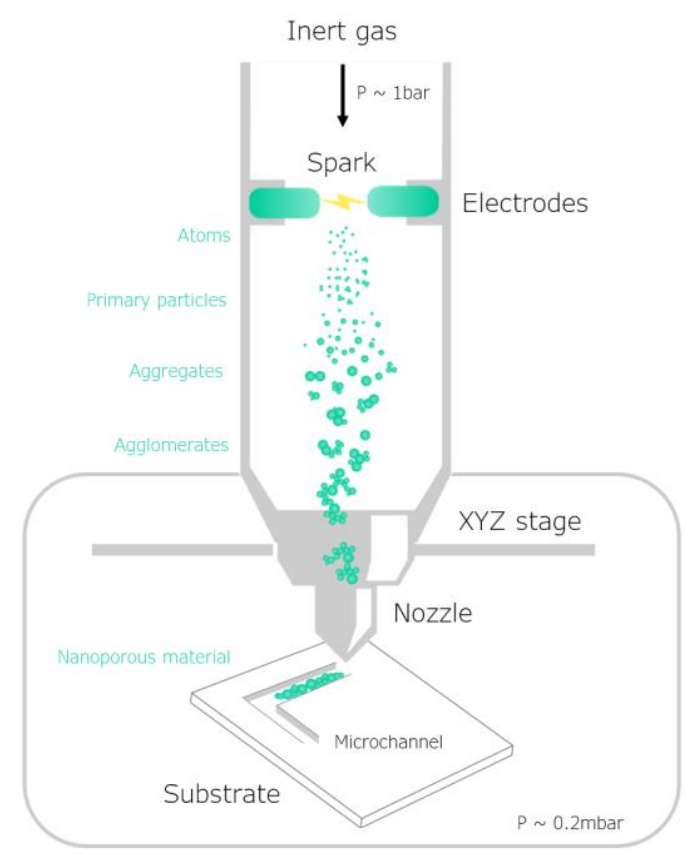

Figure 6.1 Nanostructured material printer from VSParticle (schematic). High voltages are applied between two electrodes, creating a spark and ablating atoms from the electrodes. An inert carrier gas transports the nanoparticle vapor to a nozzle. During the residence time before deposition, the atoms collide and form primary particles The particles are then deposited on the substrate containing the microchannel. As the device is placed on a mobile XYZ-stage, the position of the device relative to the nozzle is varied. 
Discharge Generation (SDG) ${ }^{20-21}$ to produce highly pure nanoparticles in an aerosol. Depending on the ablation power and the residence time in the nozzle, particles of several nanometres up to agglomerates larger than $100 \mathrm{~nm}$ can be formed. Inertial impact was used to deposit the nanoparticles from the aerosol directly into the microchannels (See Figure 6.1). In the set-up depicted in Figure 6.1, the nozzle was fixed to an XYZ-stage allowing local printing of the catalytic material, omitting post-processing steps.

The channel pattern of the device was imported into the VSP-P1 operational software to control the XYZ-stage. After placing Cu-electrodes (99.999\% purity) into the system, the $\mathrm{N}_{2}$ carrier gas (99.999\% purity) was connected and the system was warmed-up for 30 minutes. Next, the microfluidic device was loaded onto the XYZ-stage, so that the distance from the nozzle to the bottom of the channel was $1 \mathrm{~mm} \pm 0.5 \mathrm{~mm}$. The deposition chamber was then closed and the pressure reduced to 0.2 mbar. $\mathrm{Cu} / \mathrm{CuO}$ was deposited at a printing speed of $2 \mathrm{~mm} . \mathrm{min}^{-1}$. The ablation power and the carrier gas flow rate were varied between the samples, as listed in Table 6.1.

Table 6.1 Ablation power and carrier gas flow per device.

\begin{tabular}{lcc}
\hline Entry number and substrate & $\begin{array}{c}\text { Ablation power } \\
{[\mathbf{k V} ; \mathbf{m A}]}\end{array}$ & $\begin{array}{c}\text { Carrier gas flow rate } \\
{\left[\mathbf{L . m i n} \mathbf{m}^{-1}\right]}\end{array}$ \\
\hline 1. $\mathrm{SiO}_{2}$-coated & $1.3 ; 8$ & 11 \\
2. $\mathrm{SiO}_{2}$-coated & $1.3 ; 10$ & 0.7 \\
3. $\mathrm{SiO}_{2}$-coated & $1.3 ; 10$ & 0.7 \\
4. Si with native oxide & $1.3 ; 10$ & 0.7 \\
5. Si with native oxide & $1.3 ; 10$ & 0.7 \\
\hline
\end{tabular}

\subsubsection{UV-assisted degradation of methylene blue}

To evaluate the catalytic activity of the $\mathrm{Cu} / \mathrm{CuO}$ deposited in the microchannel, UV-assisted methylene blue degradation was selected as a model reaction. In the literature, it is reported that 

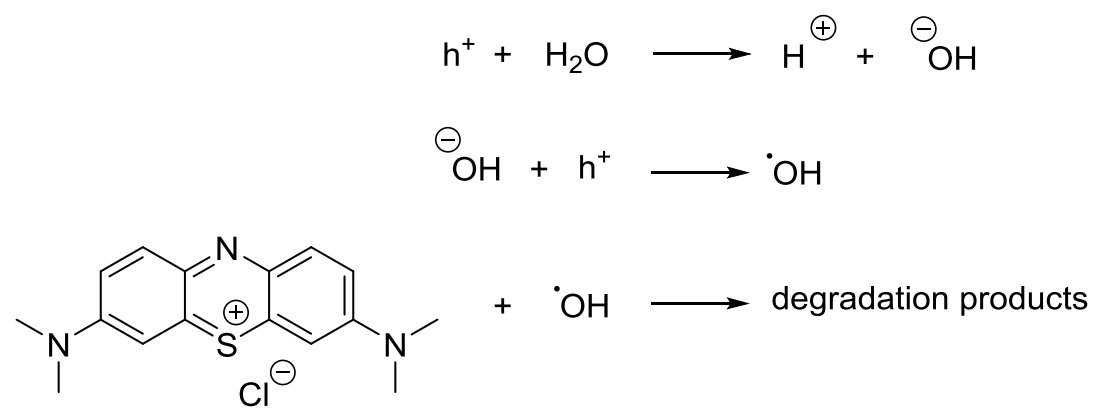

Scheme 6.1 Photocatalytic degradation of methylene blue (simplified). First, upon UV-irradation, a hole $\left(\mathrm{h}^{+}\right)$is created in the catalyst, which splits water into $\mathrm{H}^{+}$and $\mathrm{OH}^{-}$. Next, $\mathrm{OH}^{-}$reacts with another hole, forming a hydroxyl radical. This radical reacts with the methylene blue, yielding various degradation products, including $\mathrm{CO}_{2}, \mathrm{NO}_{3}{ }^{-}$, $\mathrm{NH}_{4}{ }^{+}$and $\mathrm{SO}_{4}{ }^{2-} .{ }^{1}$ Adapted from Abdellah et al. ${ }^{5}$

$\mathrm{CuO}$, when impregnated on $\mathrm{SiO}_{2}$, generates hydroxyl radicals upon $\mathrm{UV}$ irradiation, which in turn degrade methylene blue, see also Scheme 6.1. ${ }^{25-26}$

First, various methylene blue/MilliQ water solutions with concentrations between 1.6 and 0.32 mg.L $\mathrm{L}^{-1}$ were prepared for calibration purposes of the UV-VIS spectrometer. Next, a spectrum of the solution with the highest concentration was recorded in the 400 and $900 \mathrm{~nm}$ range, revealing that the maximum absorbance of methylene blue was $650 \mathrm{~nm}$.

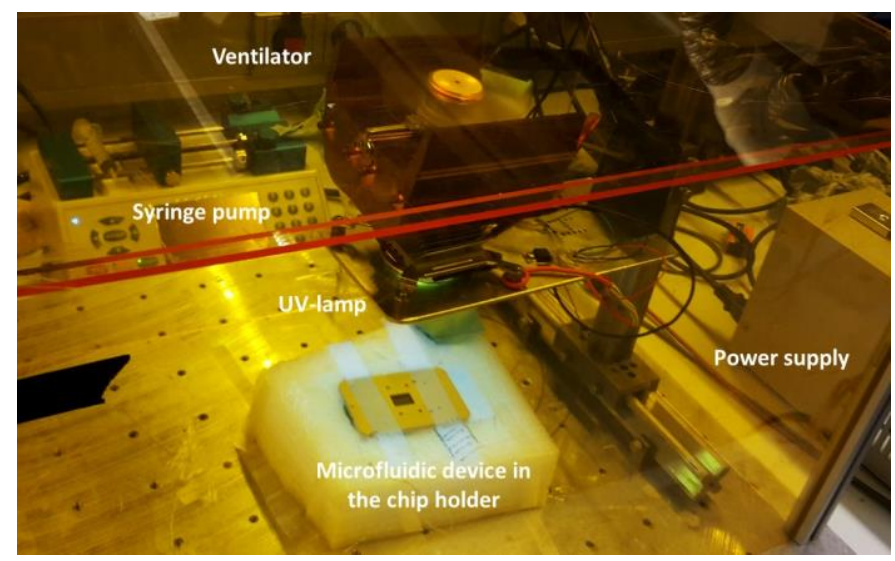

Figure 6.2 UV-set-up used for catalytic activity testing. A UV-lamp irradiated the device in the chip holder, promoting methylene blue degradation.

An UV set-up was built to perform the photocatalytic reaction (Figure 6.2). The device was placed into the same chip holder as used in Chapter 3, so that it was levelled horizontally. A $100 \mathrm{~W}$ UV LED lamp $\left(\lambda_{\max }=370 \mathrm{~nm}\right)$, placed $13 \mathrm{~cm}$ above the device was used for the irradiation of the solution flowing through the microchannel. The lamp, equipped with a ventilator for cooling, was 
connected to an external power supply. The UV section of the set-up was placed in a yellow Plexiglas box for safety. During photocatalysis, a $1.6 \mathrm{mg} . \mathrm{L}^{-1}$ methylene blue/water solution was infused at 10 or $5 \mu \mathrm{L} \cdot \mathrm{min}^{-1}$ by a syringe pump, corresponding to a residence time of 4.5 or 6 minutes. Samples were collected at the outlet of the device and analysed by UV-VIS spectroscopy. A $\mathrm{SiO}_{2}$-coated and a $\mathrm{Si}$ device with native oxide without $\mathrm{Cu} / \mathrm{CuO}$ acted as reference substrates, while MilliQ was the reference solution.

\subsection{Results and discussion}

\subsubsection{Washcoating}

Three silicon substrates containing a microfluidic channel were coated with commercial $\mathrm{TiO}_{2}$ using the dynamic washcoating technique. After calcination, SEM images were collected before and after the adhesion test. Figure 6.3 shows SEM images of several sections of a coated microfluidic channel, before and after the adhesion test (See also Appendix A1.). In all three cases, a relatively dense layer of 0.5 to $2.5-\mu \mathrm{m}$ thick was observed across the channel. No significant morphological differences in the layer were found before and after the adhesion test, suggesting good adherence of the $\mathrm{TiO}_{2}$ layer. However, in some places the titania layer was damaged, which is likely to have occurred when the UV-foil was peeled off or during dicing of the devices.

A.

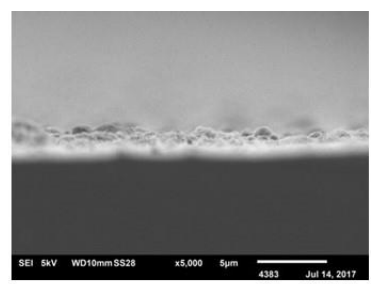

B.

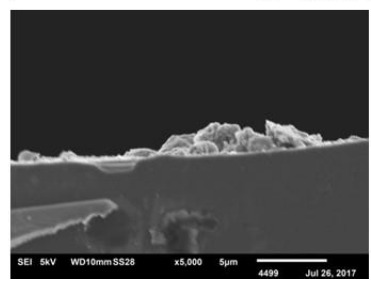

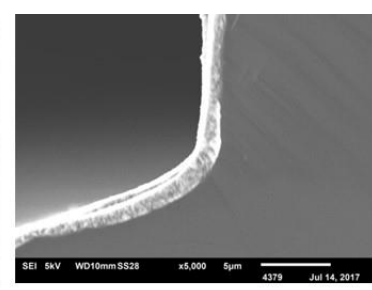

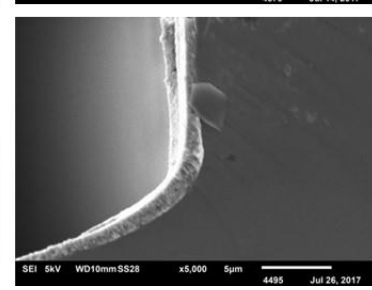

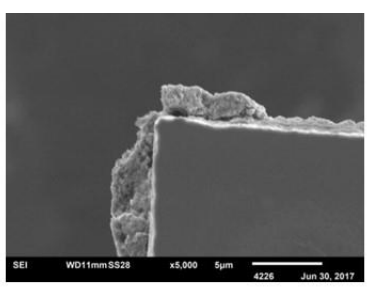

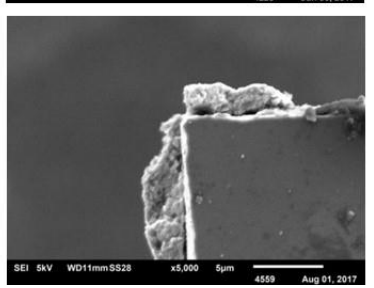

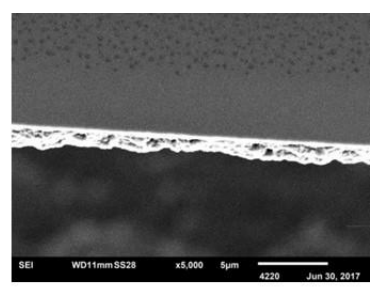

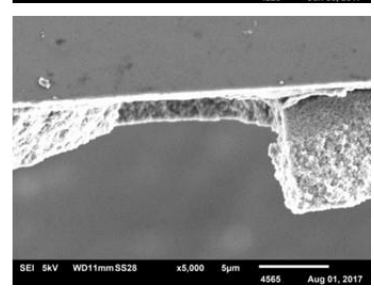

Figure 6.3. SEM analysis of a $250-\mu \mathrm{m}$ wide microchannel coated with a $5 \mathrm{wt} \% \mathrm{TiO}_{2}$ at a displacement rate of $30 \mu \mathrm{L} \cdot \mathrm{min}^{-1}$, before (A.) and after (B.) the adhesion test.

Based on the SEM images, the layer thickness was estimated (Table 6.2). Increasing the mean air displacement velocity, while keeping the same channel dimensions, resulted in a thicker titania layer. The layer thickness can be evaluated by the relation proposed by Taylor: ${ }^{27}$ 


$$
m=\left(\frac{\mu u}{\gamma}\right)^{\frac{1}{2}}
$$

where $m$ is the fraction that is deposited, $\mu$ the viscosity of the sol-gel, $u$ the mean air displacement velocity and $\gamma$ the surface tension. A higher mean displacement velocity resulted in a thicker $\mathrm{TiO}_{2}$ layer, which is in accordance with Equation 6.1.

Table 6.2. Layer thickness on the bottom corner of channels for different deposition parameters

\begin{tabular}{|c|c|c|c|}
\hline $\begin{array}{c}\text { Channel } \\
\text { width }(\mu \mathrm{m})\end{array}$ & $\begin{array}{c}\text { Air displacement flow } \\
\text { rate }\left(\mu \mathrm{L} \cdot \mathrm{min}^{-1}\right)\end{array}$ & 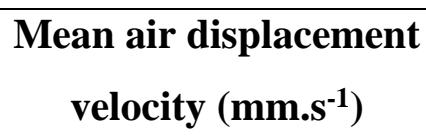 & $\begin{array}{c}\text { Layer thickness } \\
(\mu \mathrm{m})\end{array}$ \\
\hline 250 & 10 & 2.67 & $0.5-1.0$ \\
\hline 250 & 30 & 8 & $2.0-2.5$ \\
\hline 500 & 30 & 4 & $0.5-1.0$ \\
\hline
\end{tabular}

\subsubsection{Catalytic activity of $\mathrm{Cu} / \mathrm{CuO}$ nanoparticles deposited using spark ablation}

To deposit the $\mathrm{Cu} / \mathrm{CuO}$ metal active phase in the microchannel with a uniform distribution along the microchannel and good control over the particle size, the nanostructured material printer (VSPP1), developed by VSPARTICLE, was used. One $\mathrm{SiO}_{2}$-coated and one $\mathrm{Si}$ device with native oxide were used to evaluate the catalytic activity of the $\mathrm{Cu} / \mathrm{CuO}$ particles towards $\mathrm{UV}$-assisted degradation of methylene blue.

Table 6.3 presents the methylene blue conversion for the different devices, with $\mathrm{Cu} / \mathrm{CuO}$ nanoparticles deposited on some of them. For the devices without $\mathrm{Cu} / \mathrm{CuO}$, a conversion around 0.5 was achieved, whereas for the devices with $\mathrm{Cu} / \mathrm{CuO}$ a lower conversion was determined (with the exception of one $\mathrm{Cu} / \mathrm{CuO} / \mathrm{SiO}_{2}$ measurement). However, overall, a large spreading was observed in the conversion data, from which no robust conclusion can be drawn. It might be related to the difference in light intensity that is reaching the catalytic layer. For further research a light sensor should be included in the microfluidic reactor near the catalytic layer to locally determine the light intensity. Still, these results suggest that the presence of $\mathrm{Cu} / \mathrm{CuO}$ did not catalyze the methylene blue degradation, in contrast to our expectations. 
Table 6.3 Methylene blue conversion at different flow rates and with different substrates, with and without $\mathrm{Cu} / \mathrm{CuO}$ nanoparticles

\begin{tabular}{lccc}
\hline Substrate & $\begin{array}{c}\text { Residence time } \\
{[\mathbf{m i n}]}\end{array}$ & $\begin{array}{c}\text { A } \\
{[-]}\end{array}$ & Calculated conversion \\
\hline $\begin{array}{l}\text { Methylene blue } \\
\text { before reaction }\end{array}$ & 4.5 & 0.589 & \\
$\mathrm{Cu} / \mathrm{CuO} / \mathrm{SiO}_{2}$ & 4.5 & 0.270 & 0.58 \\
& 6 & 0.434 & 0.28 \\
& 4.5 & 0.334 & 0.46 \\
$\mathrm{Cu} / \mathrm{CuO} / \mathrm{Si}$ with native oxide & 4.5 & 0.407 & 0.35 \\
& 6 & 0.473 & 0.24 \\
$\mathrm{SiO}{ }_{2}$-coated & 4.5 & 0.493 & 0.24 \\
$\mathrm{Si}$ with native oxide & 4.5 & 0.311 & 0.50 \\
\hline
\end{tabular}

\subsubsection{SEM analysis of the $\mathrm{Cu} / \mathrm{CuO}$ layer}

To characterize the morphology of the $\mathrm{Cu} / \mathrm{CuO}$ layer before and after bonding, SEM images were recorded of device $1\left(\mathrm{SiO}_{2}\right.$-coated see also Table 6.1) at a magnification of 6500x (A.) and 50000x (B.). The images revealed a highly porous layer with cluster sizes around $120 \mathrm{~nm}$, which were homogeneously distributed in the microchannel, see Figure 6.4. Furthermore, as these pictures were taken after bonding, it was concluded that the layer was not affected by the used anodic bonding procedure.

SEM analysis and energy-dispersive X-ray (EDX) spectroscopy were performed on the remaining samples which were prepared on different days, before and after catalysis. Figure 6.5 presents two different $\mathrm{SiO}_{2}$-coated substrates, with in $\mathrm{A}$. and $\mathrm{B}$. the substrate before bonding and reaction and in C. the substrate after reaction. Directly after deposition, nanoparticles around $5 \mathrm{~nm}$ in diameter were uniformly distributed over the microchannel, while almost no nanoparticles were observed after catalysis. The decrease in the amount of nanoparticles could have two causes: either $\mathrm{Cu} / \mathrm{CuO}$ was not properly deposited on this particular substrate, or nanoparticles were lost upon liquid 

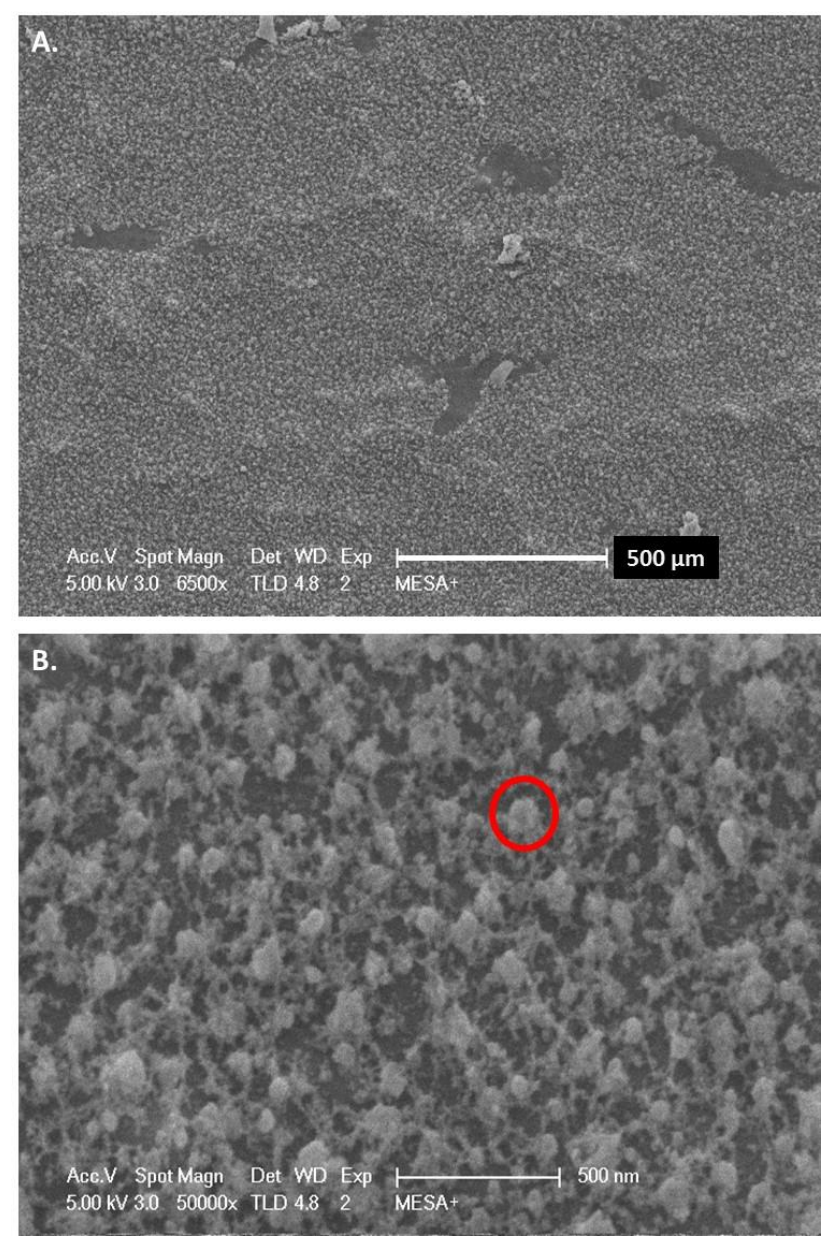

Figure 6.4. $\mathrm{SEM}$ analysis of the $\mathrm{Cu} / \mathrm{CuO}$ layer deposited in a $\mathrm{SiO}_{2}$-coated microchannel at a 6500 and a 50000 magnification, after bonding. A highly porous layer was deposited with $\mathrm{Cu} / \mathrm{CuO}$-cluster (red circle) around 120 $\mathrm{nm}$ in size.

perfusion, which would indicate poor adhesion. For the $\mathrm{Cu} / \mathrm{CuO} / \mathrm{Si}$ device with native oxide similar results were obtained.

Besides nanoparticles, larger $\mathrm{Cu} / \mathrm{CuO}$ clusters (of $260 \mathrm{~nm}$ and $2700 \mathrm{~nm}$ in size, Figure 6.6) were observed on both the $\mathrm{Si}$ with native oxide and $\mathrm{SiO}_{2}$-coated substrates, which could have formed already in the spark ablation set-up as a result of the low flow rate of the carrier gas and low residence time. Although the 5-nm particles were too small for EDX analysis, elemental analysis could be performed on these larger clusters. EDX analysis confirmed the presence of $\mathrm{Cu}(50 \mathrm{wt} \%$ ), but also revealed oxygen (17\%), Si (8 wt $\%$ ) and C (22 wt $\%)$. The presence of oxygen might indicate either $\mathrm{CuO}$ or native oxide on the $\mathrm{Si}$-substrate. Carbon contamination possibly occurred during the installation of the electrodes in the spark ablation set-up. 

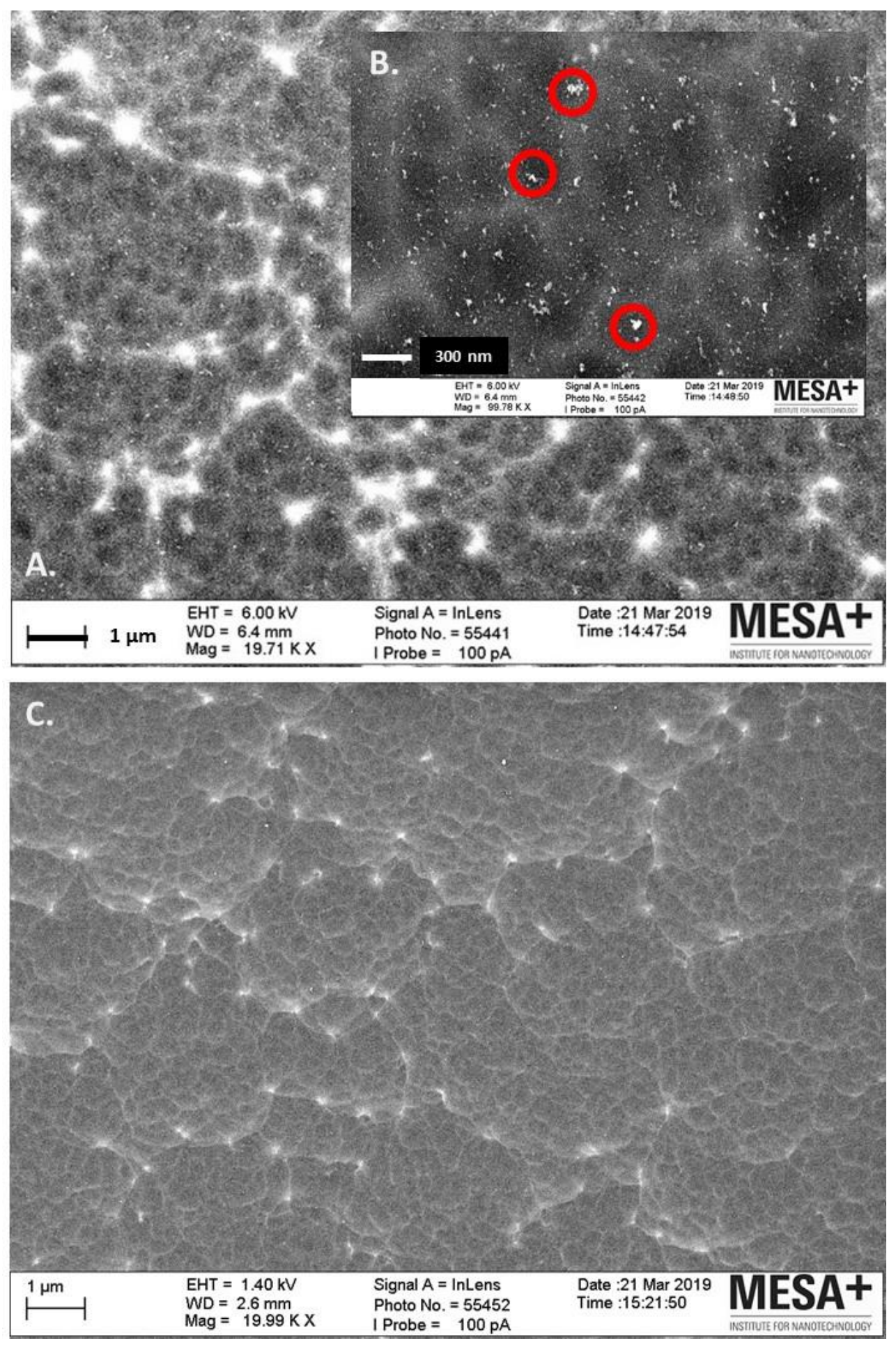

Figure 6.5. SEM analysis of the $\mathrm{Cu} / \mathrm{CuO}$ layer deposited in $\mathrm{SiO}_{2}$-coated microchannels of two different samples, before (A. and B.) and after catalysis (C.). Small clusters around $40 \mathrm{~nm}$ in diameter are indicated in B. Before reaction, particles around $5 \mathrm{~nm}$ in diameter were detected, whereas no particles were visible after catalysis. 

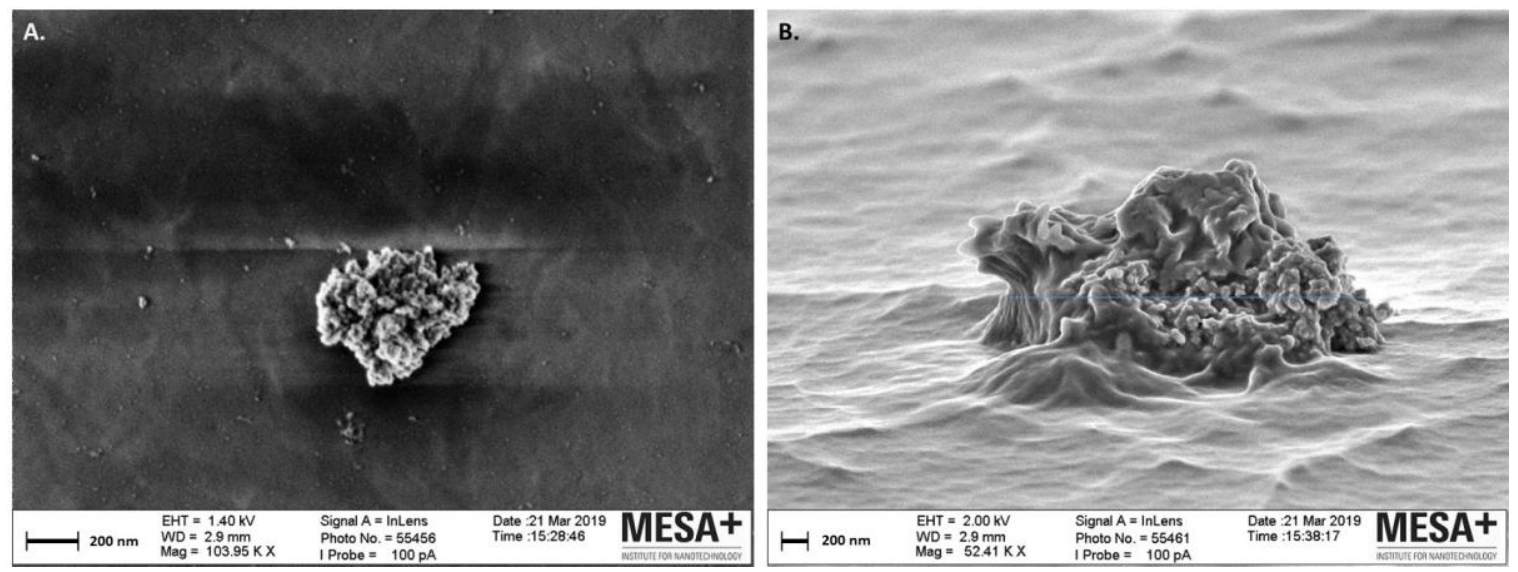

Figure 6.6. $\mathrm{SEM}$ images of the $\mathrm{Cu} / \mathrm{CuO}$ clusters on the $\mathrm{Si}$ substrate with native oxide before bonding and reaction, with in $\mathrm{A}$. a $\mathrm{Cu} / \mathrm{CuO}$ cluster of $260-\mathrm{nm}$ in diameter, top view and a $2700-\mathrm{nm}$ wide $\mathrm{Cu} / \mathrm{CuO}$ cluster side view in B.

\subsection{Conclusion}

In this chapter, washcoating, a chemical deposition technique, and spark ablation, a physical deposition technique, were investigated to deposit a $\mathrm{TiO}_{2}$ catalyst support layer and a $\mathrm{Cu} / \mathrm{CuO}$ metal active phase, respectively, in $\mathrm{Si}$ with native oxide or $\mathrm{SiO}_{2}$-coated microchannels. $\mathrm{A} \mathrm{TiO}_{2}$ layer up to $2.5 \mu \mathrm{m}$ in thickness could be deposited with good adhesion to the microchannel wall. The uniformity of the layer, however, must still be improved. $\mathrm{Cu} / \mathrm{CuO}$ deposition by spark ablation resulted in 5-nm particles, which were homogeneously distributed over the substrate surface, compatible with anodic bonding. UV-assisted methylene degradation suggested that the $\mathrm{Cu} / \mathrm{CuO}$ particles might be inactive for this particular reaction, although the data was obtained with poor reproducibility. SEM analysis revealed that most $\mathrm{Cu} / \mathrm{CuO}$-particles were either not properly deposited, or removed after catalysis. Still, the combination of these two methods is promising for controlled catalyst deposition in microchannels.

\section{Acknowledgements}

I would like to thank Erilia Yusnitha and Aura Visan for the collaboration on the washcoating procedure. I also thank VSParticle B.V. for the collaboration on the physical deposition using spark ablation, Vincent de Boer for building the UV-set-up and Mark Smithers for the high resolution SEM imaging. 


\section{References}

1. Houas, A.; Lachheb, H.; Ksibi, M.; Elaloui, E.; Guillard, C.; Herrmann, J.-M., Photocatalytic degradation pathway of methylene blue in water. Applied Catalysis B: Environmental 2001, 31 (2), 145-157.

2. Chheda, J. N.; Huber, G. W.; Dumesic, J. A., Liquid-phase catalytic processing of biomassderived oxygenated hydrocarbons to fuels and chemicals. Angewandte Chemie-International Edition 2007, 46 (38), 7164-7183.

3. Davda, R. R.; Shabaker, J. W.; Huber, G. W.; Cortright, R. D.; Dumesic, J. A., A review of catalytic issues and process conditions for renewable hydrogen and alkanes by aqueous-phase reforming of oxygenated hydrocarbons over supported metal catalysts. Applied Catalysis BEnvironmental 2005, 56 (1-2), 171-186.

4. Davda, R. R.; Shabaker, J. W.; Huber, G. W.; Cortright, R. D.; Dumesic, J. A., Aqueousphase reforming of ethylene glycol on silica-supported metal catalysts. Applied Catalysis B: Environmental 2003, 43 (1), 13-26.

5. Abdellah, M. H.; Nosier, S. A.; El-Shazly, A. H.; Mubarak, A. A., Photocatalytic decolorization of methylene blue using TiO2/UV system enhanced by air sparging. Alexandria Engineering Journal 2018, 57 (4), 3727-3735.

6. Iliuta, I.; Hamidipour, M.; Schweich, D.; Larachi, F., Two-phase flow in packed-bed microreactors: Experiments, model and simulations. Chem. Eng. Sci. 2012, 73, 299-313.

7. He, W.; Fang, Z.; Zhang, K.; Tu, T.; Lv, N.; Qiu, C.; Guo, K., A novel micro-flow system under microwave irradiation for continuous synthesis of 1,4-dihydropyridines in the absence of solvents via Hantzsch reaction. Chem. Eng. J. 2018, 331, 161-168.

8. Munirathinam, R.; Huskens, J.; Verboom, W., Supported Catalysis in Continuous-Flow Microreactors. Adv. Synth. Catal. 2015, 357 (6), 1093-1123.

9. Zhu, L.; Fu Tan, C.; Gao, M.; Ho, G. W., Design of a Metal Oxide-Organic Framework (MoOF) Foam Microreactor: Solar-Induced Direct Pollutant Degradation and Hydrogen Generation. Adv. Mater. 2015, 27 (47), 7713-7719.

10. Chen, G.; Zhu, X.; Chen, R.; Liao, Q.; Ye, D.; Feng, H.; Liu, J.; Liu, M., Gas-liquid-solid monolithic microreactor with Pd nanocatalyst coated on polydopamine modified nickel foam for nitrobenzene hydrogenation. Chem. Eng. J. 2018, 334, 1897-1904. 
11. Tanimu, A.; Jaenicke, S.; Alhooshani, K., Heterogeneous catalysis in continuous flow microreactors: A review of methods and applications. Chem. Eng. J. 2017, 327, 792-821.

12. D'Angelo, M. F. N.; Ordomsky, V.; Paunovic, V.; van der Schaaf, J.; Schouten, J. C.; Nijhuis, T. A., Hydrogen Production through Aqueous-Phase Reforming of Ethylene Glycol in a Washcoated Microchannel. ChemSusChem 2013, 6 (9), 1708-1716.

13. Schlesinger, M., Electroplating. In Kirk-Othmer Encyclopedia of Chemical Technology.

14. Krebs, F. C., Fabrication and processing of polymer solar cells: A review of printing and coating techniques. Sol. Energy Mater. Sol. Cells 2009, 93 (4), 394-412.

15. Kaiser, N., Review of the fundamentals of thin-film growth. Appl. Opt. 2002, 41 (16), 3053-3060.

16. Echave, F. J.; Sanz, O.; Montes, M., Washcoating of micro-channel reactors with PdZnO catalyst for methanol steam reforming. Applied Catalysis a-General 2014, 474, 159-167.

17. Stefanescu, A.; van Veen, A. C.; Mirodatos, C.; Beziat, J. C.; Duval-Brunel, E., Wall coating optimization for microchannel reactors. Catal. Today 2007, 125 (1-2), 16-23.

18. Ambrosetti, M.; Balzarotti, R.; Cristiani, C.; Groppi, G.; Tronconi, E., The Influence of the Washcoat Deposition Process on High Pore Density Open Cell Foams Activation for CO Catalytic Combustion. Catalysts 2018, 8 (11).

19. Van Bui, H.; Grillo, F.; van Ommen, J. R., Atomic and molecular layer deposition: off the beaten track. Chem. Commun. 2017, 53 (1), 45-71.

20. Hontanon, E.; Palomares, J. M.; Stein, M.; Guo, X. A.; Engeln, R.; Nirschl, H.; Kruis, F. E., The transition from spark to arc discharge and its implications with respect to nanoparticle production. J. Nanopart. Res. 2013, 15 (9).

21. Tabrizi, N. S.; Ullmann, M.; Vons, V. A.; Lafont, U.; Schmidt-Ott, A., Generation of nanoparticles by spark discharge. J. Nanopart. Res. 2009, 11 (2), 315-332.

22. Makhlouf, A. S. H., 1 - Current and advanced coating technologies for industrial applications. In Nanocoatings and Ultra-Thin Films, Makhlouf, A. S. H.; Tiginyanu, I., Eds. Woodhead Publishing: 2011; pp 3-23.

23. Ravenelle, R. M.; Copeland, J. R.; Kim, W.-G.; Crittenden, J. C.; Sievers, C., Structural Changes of $\gamma$-Al2O3-Supported Catalysts in Hot Liquid Water. ACS Catalysis 2011, 1 (5), 552561. 
24. Xiong, H.; Pham, H. N.; Datye, A. K., Hydrothermally stable heterogeneous catalysts for conversion of biorenewables. Green Chemistry 2014, 16 (11), 4627-4643.

25. Batista, A. P. L.; Carvalho, H. W. P.; Luz, G. H. P.; Martins, P. F. Q.; Goncalves, M.; Oliveira, L. C. A., Preparation of $\mathrm{CuO} / \mathrm{SiO} 2$ and photocatalytic activity by degradation of methylene blue. Environmental Chemistry Letters 2010, 8 (1), 63-67.

26. Kakhki, R. M.; Ahsani, F.; Mir, N., Enhanced photocatalytic activity of CuO-SiO2 nanocomposite based on a new $\mathrm{Cu}$ nanocomplex. Journal of Materials Science-Materials in Electronics 2016, 27 (11), 11509-11517.

27. Taylor, G. I., Deposition of a viscous fluid on the wall of a tube. J. Fluid Mech. 2006, 10 (2), 161-165. 


\section{A6.1 SEM analysis of washcoated silicon substrates}

A.
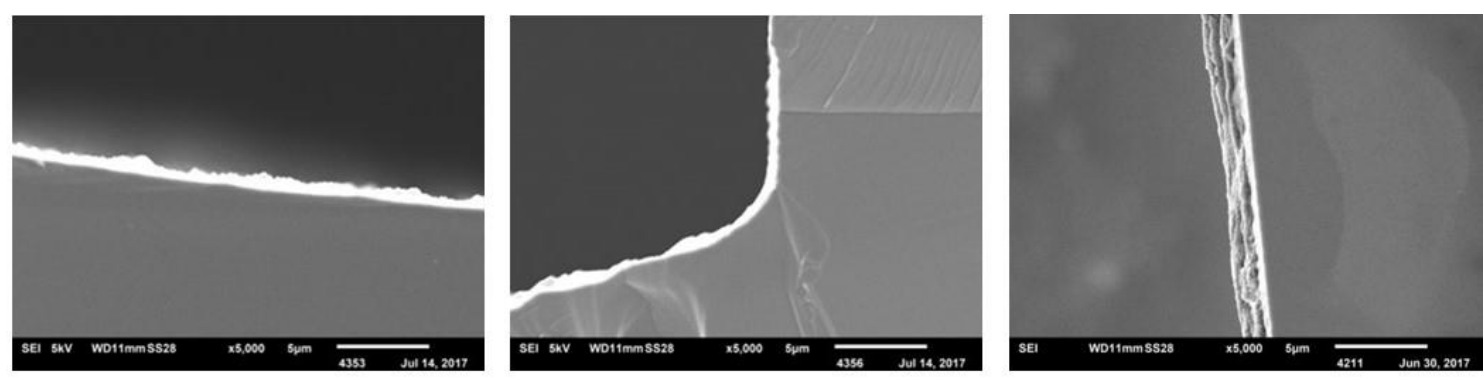

B.
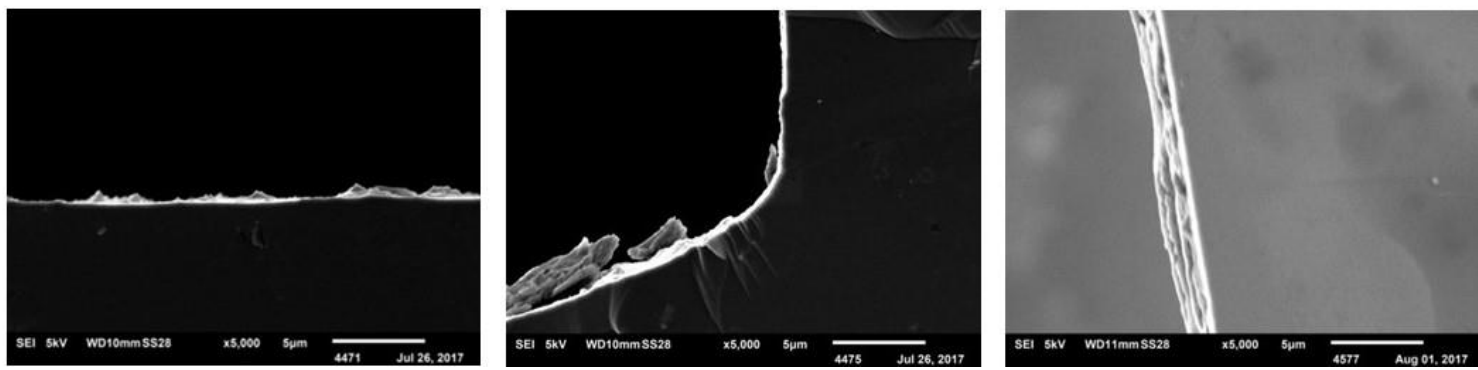

Figure A6.1. SEM analysis of 250- $\mu \mathrm{m}$ wide microchannel coated with $5 \mathrm{wt} \% \mathrm{TiO}_{2}$ at a displacement rate of 10 $\mu \mathrm{L} . \mathrm{min}^{-1}$, before (A) and after (B) the adhesion test.

A.
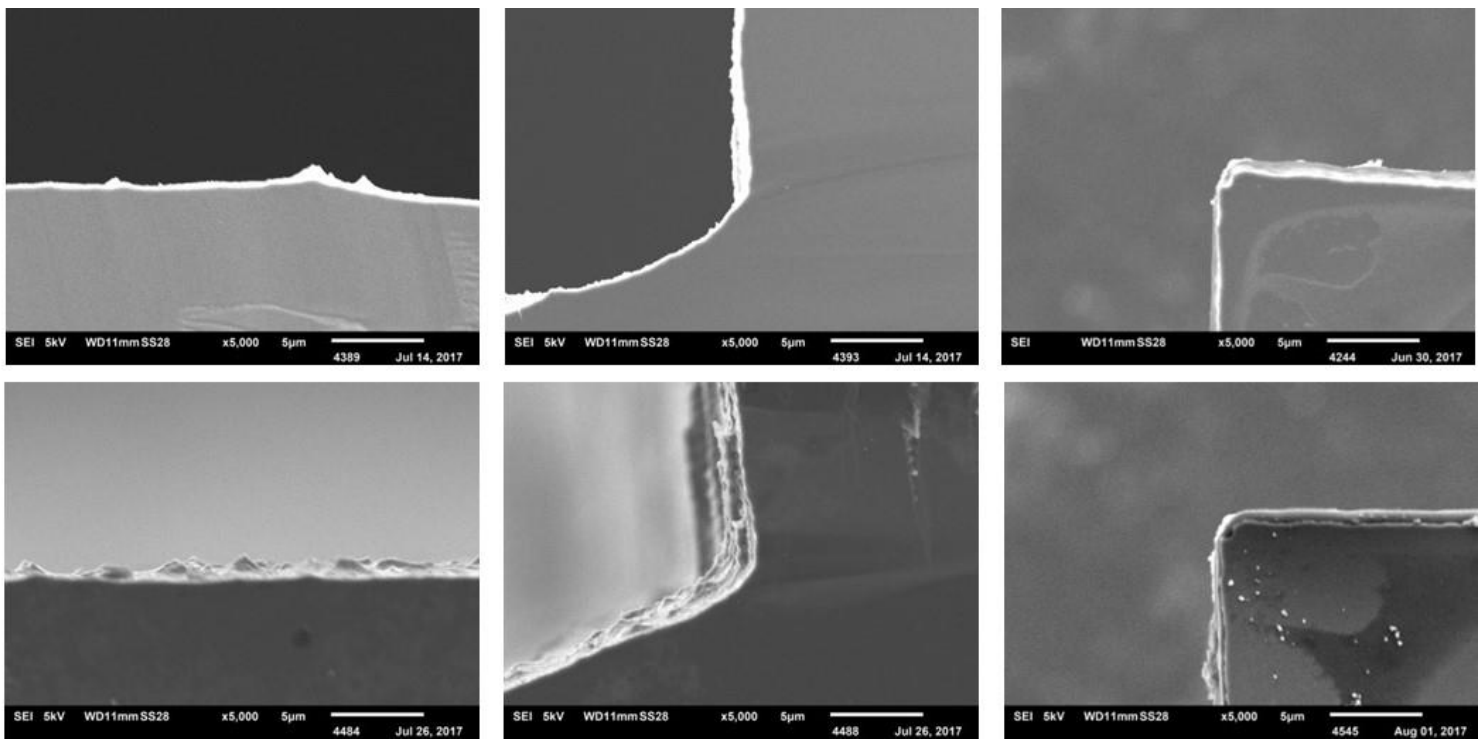

Figure A6.2. SEM analysis of 500- $\mu \mathrm{m}$ wide microchannel coated with $5 \mathrm{wt} \% \mathrm{TiO}_{2}$ at a displacement rate of 30 $\mu \mathrm{L} \cdot \mathrm{min}^{-1}$, before (A) and after (B) the adhesion test. 
CHAPTER 6 


\title{
Fabrication of a gas/liquid microseparator with ultra-low dead volume
}

\begin{abstract}
Separation of the gas and the liquid phases at a reactor outlet is relatively straightforward at a large scale using, for example, gravity-based methods. In contrast, downstream processing of gaseous and liquid products leaving a microreactor, however, gives rise to many different challenges, especially when inline analysis is performed after the reaction. At the microscale, throughput is typically low and valuable data are lost if there is significant dead volume. For example, for kinetic analysis by gas chromatography, concentrations are averaged over longer time scales in order to collect enough sample to overcome the detection limit. In this chapter, the fabrication of a modular microseparator based on a micropillar membrane, while minimizing the dead volume is described.
\end{abstract}




\subsection{Introduction}

In Aqueous-Phase Reforming (APR), a combination of gases is produced in the bulk liquid phase. ${ }^{1-}$ ${ }^{3}$ Depending on the reaction coordinates and the catalyst selectivity towards the desired hydrogen, products like $\mathrm{CO}, \mathrm{CO}_{2}, \mathrm{CH}_{4}$ and short $\mathrm{C}$-chain carbohydrates and derivatives thereof can be found. ${ }^{4-5}$ For kinetic analysis, but also to obtain pure hydrogen, the first step in the work-up of the reaction mixture is to separate the gaseous products from the liquid phase reaction mixture.

On a large scale, separation occurs, for example, via gravity settling, where the gas/liquid mixture enters a vessel in which light gases rise to the top of the vessel, while more dense and heavier liquids remain on the bottom. ${ }^{6}$ Other industrial gas/liquid separators are based on membranes, ${ }^{7}$ or a combination of gravitational and centrifugal forces (cyclone separators). ${ }^{8}$

However, for a microfluidic reactor with a typical throughput in the order of microliters per minute, these separator designs are impractical. Microreactors are particularly interesting for research purposes and analysis of small sample volumes, ${ }^{9-11}$ such as elucidation of reaction mechanisms and kinetic studies. ${ }^{12-13}$ For these purposes, it is very important that the gas/liquid separation is both fast and efficient for the small throughput volume, so that small concentration differences can be measured with good temporal resolution. In this respect, the dead volume of the device and its size plays an important role: gas and/or liquid might accumulate in these spaces, resulting in loss of information due to sample averaging, ${ }^{14}$ and/or chromatographic peak broadening. ${ }^{15}$

In the literature, several modular microfluidic devices for gas/liquid separation have been presented, often based on membrane technology. A relatively straightforward design was presented by Xu and co-workers, ${ }^{16}$ where a hydrophobic acrylic co-polymer membrane was placed on top of the fluidic channel. A more intricate device was designed by Lochovksy et al. ${ }^{17}$ where gas bubbles were first trapped by an array of pillars in a chamber of 1-mm in diameter and subsequently removed via a vacuum channel after permeating through a PDMS membrane. This type of devices is, however, mechanically fragile, as a result of the membrane and they require a delicate assembly procedure. Furthermore, the gas bubbles must have a certain size (bubble length must exceed the channel diameter), ${ }^{16}$ limiting the range of void fractions that can be separated.

More mechanically robust microseparators are based on small side-channels that are positioned perpendicular to the main fluidic channel, as is the case in a T-junction. The multiphase flow passes 
the junction where the liquid is extracted. The gas plug only penetrates temporarily into the side channel, after which it regains its shape and continues flowing through the main channel. This separation method is only possible when the pressure difference between the gas and the liquid phase remains smaller than the surface tension. ${ }^{18}$ A more sophisticated example of this principle was reported by Zenith et al. ${ }^{19}$ where the extraction of the liquid was aided by the hydrophilicity of the side capillary and the hydrophobic main channel.

These microfluidic systems are all limited in terms of volumetric flow rate and void fraction. ${ }^{19}$ Therefore, multiple capillary designs have been investigated. ${ }^{20} \mathrm{~A}$ variation on this capillary channel approach is a micropillar membrane, for which the separation parameters have been modeled in detail. ${ }^{21-22}$ An array of micropillars form a barrier for the gas, while allowing the liquid to permeate. When the liquid fraction in the gas phase is decreasing as a result of the separation, gas plugs will eventually merge and complete separation will be achieved. In the simulations, breakthrough of the gas phase into the liquid phase channel was observed only when the capillary pressure limit was exceeded by the gas bubbles. ${ }^{22}$

Although the devices described by $\mathrm{Xu}^{21}$ and $\mathrm{An}^{22}$ showed promising simulation results, to the best of our knowledge, such micropillar designs have not yet been evaluated experimentally. In this chapter, the fabrication and early testing for such a modular microseparator is described. Special attention was given to the configuration of the in- and outlets when designing the separation module, since dead volume typically forms at those locations.

\subsection{Design and fabrication}

\subsubsection{Design requirements}

With the final application in mind, the gas/liquid separator must be able to handle a wide range of void fractions, varying from bubbly flow to Taylor flow (see also Chapter 3), and effectively separate at low flow rates starting at $10 \mu \mathrm{L} \cdot \mathrm{min}^{-1}$. Furthermore, the device should be able to process high flow rates (up to $1 \mathrm{~mL} \cdot \mathrm{min}^{-1}$ ) without compromising its mechanical stability. To be compatible with a wide range of chemical reactions, the materials of which the device is made must be chemically inert. For kinetic applications, the dead volume is of utmost importance and must be below $0.1 \mu \mathrm{L}$, which corresponds to $1 \%$ of the minimum flow rate that the microseparator 
must be able to handle. The modularity of the device would allow connecting this microseparator to the outlet of any microreactor.

\subsubsection{Channel and micropillar parameters}

Based on the work of $\mathrm{Xu}^{21}$ and $\mathrm{An},{ }^{22}$ the microseparator consists of one straight, symmetric channel of 8000 microns in length with a micropillar structure downstream, Figure 7.1. All parameters and their values are listed in Table 7.1. All the features were fabricated in a silicon substrate, which was covered by a glass layer. As the micropillars have the same height as the microchannel itself, the pillars were connected both to the bottom and the glass cover of the channel, giving the pillar structures extra mechanical strength.

Table 7.1 Microseparator parameters and their corresponding symbols and values, as depicted in Figure 7.1.

\begin{tabular}{llcc}
\hline Parameter & & Symbol & Value $(\boldsymbol{\mu m})$ \\
\hline Channel height & & $H$ & 100 \\
Main channel & Width & $D$ & 201 \\
& Length & $L$ & 3000 \\
Separation section & Separation length & $L_{s}$ & 5000 \\
& Gas channel width & $D_{G}$ & 38 \\
& Liquid channel width & $D_{L}$ & 115 \\
Micropillars & Width & $P_{y}$ & 5 \\
& Length & $P_{x}$ & 5 \\
& Spacing & $P S_{x}$ & 5 \\
\hline
\end{tabular}

\subsubsection{In- and outlets}

The device required at least three connections: one inlet for the gas/liquid stream, one outlet for the gas phase and one outlet for the liquid phase. To reduce the number of required connections, 


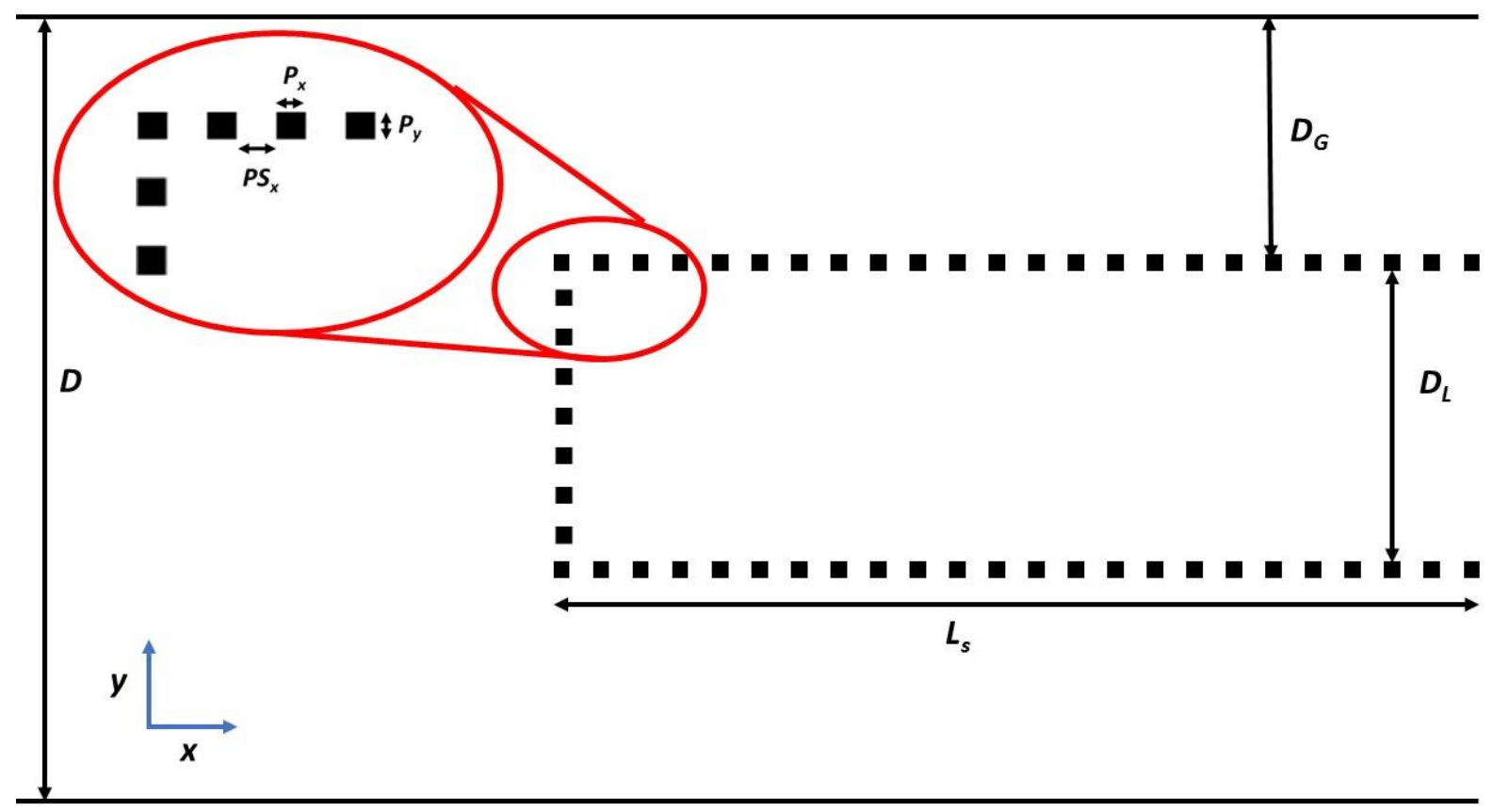

Figure 7.1 Schematic of the separation micropillar membrane, with the flow direction from the left to the right (top view). $D, D_{G}, D_{L}$ are the widths of the main channel, the gas channel and the liquid channel, respectively. $P_{s}$, $P_{y}$ and $P S_{x}$ are pillar dimensions in the $x$ and $y$ direction and the pillar spacing.

the gas outlet streams were recombined into a single outlet (Figure 7.2), with the added advantage that an outlet stream of larger volume is more likely to exceed the detection limit of the intended analysis method.

Commonly in microfluidic devices, the in- and outlet tubings are perpendicular to the channel, so that the liquid flow must change direction, which can give rise to dead volume in the sharp corners. In this design, the gas/liquid inlet and the gas outlets were in the same xy-plane as the channel, therefore circumventing this effect, ${ }^{23}$ while the liquid outlet was created through the silicon

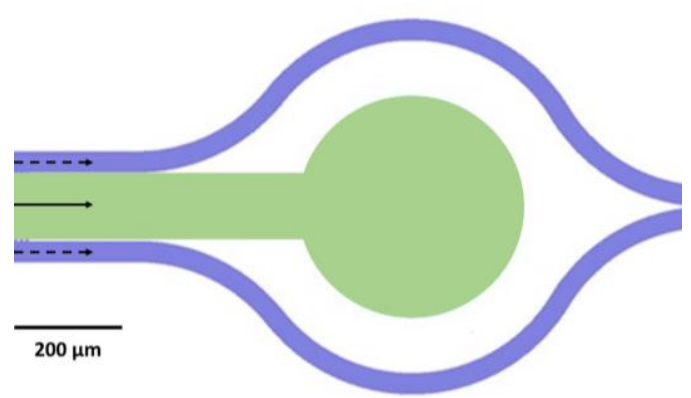

Figure 7.2 Outlet, with in green the liquid outlet and in purple the combined gas outlets.

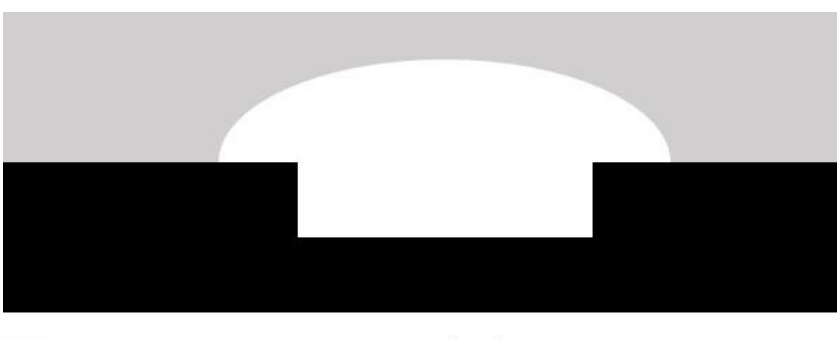

Glass
Figure 7.3 Cross-section of the in- and outlet configuration without a capillary, cross-section. Not to scale. 
substrate. Specifically, fused silica capillaries (OD $360 \mu \mathrm{m}$; ID $200 \mu \mathrm{m}$ ) were glued into the device for which dedicated structures were included in the design. To align the capillary with the gas outlet channel, both the silicon layer containing the channel and the glass cover were processed (Figure 7.3).

\subsubsection{Processing the silicon wafer}

As previously mentioned, the device itself consisted of two layers. All the structures were manufactured in silicon (with a thin native oxide layer), which was subsequently covered by a glass wafer. These materials are compatible with a wide range of chemicals and structures are usually better defined in silicon than in for example polydimethylsiloxane (PDMS). The transparency of the glass allowed optical monitoring of the separation process.

As the channels for the capillaries are deeper than the main and separation channel, a buried mask was used. First, a 500-nm thick silicon oxide layer was grown on the silicon wafer $(10-\mathrm{cm}$ diameter, $\langle 100\rangle$, p/boron-doped, 525- $\mu \mathrm{m}$ thick, Okmetic) by wet oxidation. Secondly, after a photolithography step, all structures were plasma etched into the $\mathrm{SiO}_{2}$ layer. Afterwards, the photoresist used for photolithography was stripped from the silicon substrate. In the second photolithography step, the capillary pattern was transferred onto the substrate, followed by DRIE etching $80 \mu \mathrm{m}$-deep into the silicon. After stripping the photoresist, a last and final DRIE etching step was performed with only the $\mathrm{SiO}_{2}$ acting as a hard mask. In this step, the separation channel was etched $100 \mu \mathrm{m}$-deep into the silicon, while the capillary channel reached the desired $180-\mu \mathrm{m}$ depth.

The outlet for the separated liquid stream was fabricated on the back side of the silicon wafer. After photolithography, the hole was DRIE etched completely through the wafer, reaching the channel. Afterwards the photoresist was stripped, and the wafer was submerged in 50\% $\mathrm{HF}$ to remove any remaining $\mathrm{SiO}_{2}$. To remove any fluorocarbon residue deposited during the DRIE process, a fresh 200-nm oxide layer was grown and subsequently stripped in $50 \% \mathrm{HF}$. See Appendix A7.1 for the fabrication details. 


\subsubsection{Processing the glass top layer}

The glass cover (MEMPax, 10-cm diameter, 500- $\mu \mathrm{m}$ thick, Schott AG) did not require as much processing as the silicon wafer: only the capillary in- and outlets were powder-blasted (Figure 7.3). One side of the wafer was covered with Adwill dicing foil for protection, whereas the desired pattern was transferred onto the substrate via lithography with Harke i-HE foil. $\mathrm{Al}_{2} \mathrm{O}_{3}$ particles (29 $\mu \mathrm{m}$ in diameter) were used to powder blast the features into the glass. Next, the foils were manually removed and the wafer was cleaned in an ultrasonic bath. See Appendix A7.1 for the fabrication details.

\subsubsection{Post-processing}

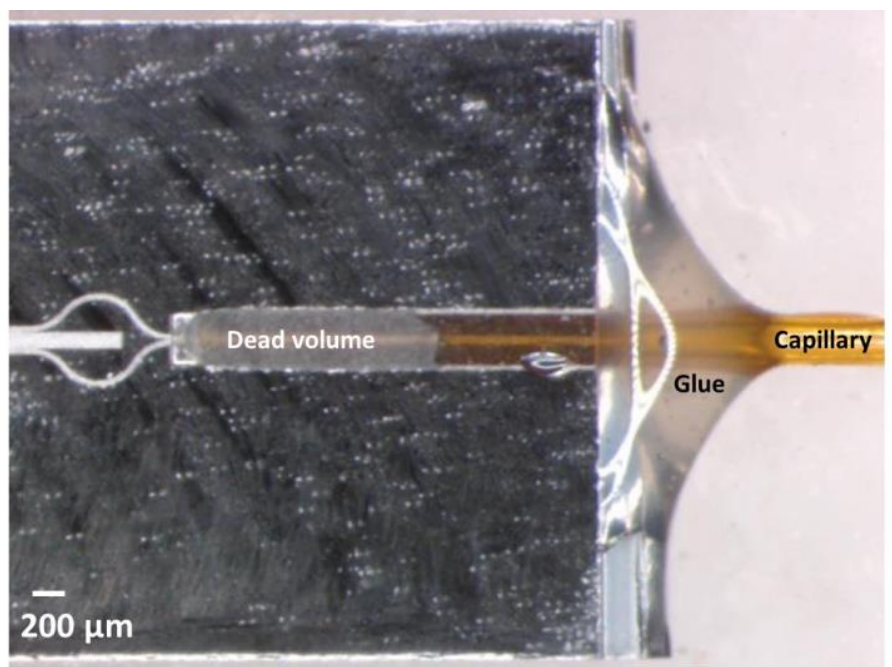

Figure 7.4 Outlet of the device with glued-in capillary. The glue has not fully penetrated into the capillary canal, forming a dead volume.
The post-processing consisted of bonding the wafers, followed by dicing of the wafer into individual chips and gluing the capillaries into the dedicated channels. First, both wafers were cleaned in piranha for 15 minutes, followed by alignment of the silicon and glass wafers and anodic bonding at $673 \mathrm{~K}$ and $1000 \mathrm{~V}$ in vacuum. Secondly, the wafer stack was diced to obtain the individual devices. This step is not straightforward, as dicing cannot be

done along the in- and outlets (Figure 7.5): dicing would inevitably lead to particle formation that could clog the in- and outlets. Therefore, the in- and outlets of the devices were placed inlet to outlet on the wafer. First, a row of devices was diced free from the rest of the wafer. Secondly, a small indent as a breaking line was diced along the in- and outlets, to finally obtain the individually devices.

Finally, the capillaries were glued into the devices. The capillaries were cut to size using a diamond cutter resulting in a straight plane, cleaned with isopropyl alcohol and inserted into the device. 


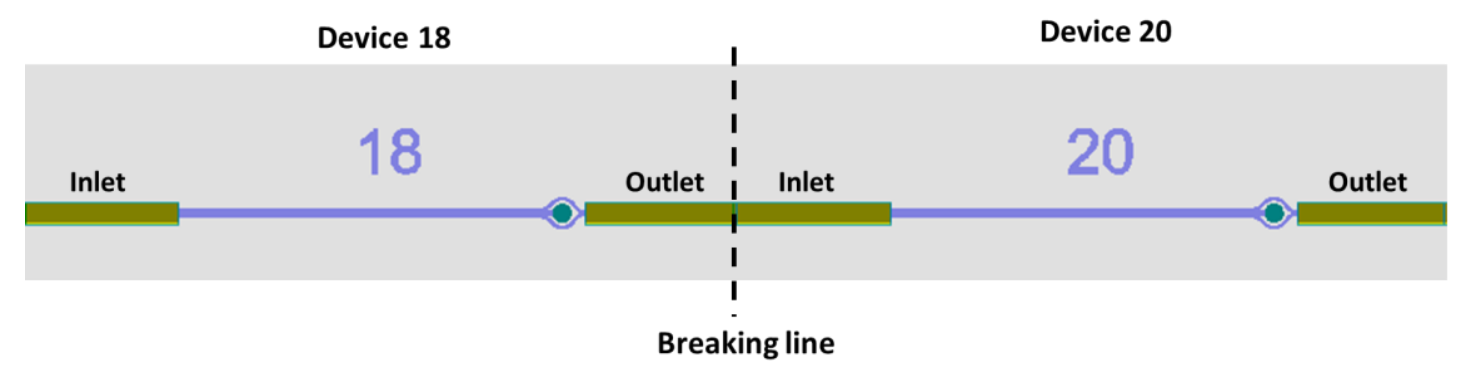

Figure 7.5 Two devices are placed inlet to outlet in the wafer design. The devices are first diced along the long side of the devices, followed by gentle breaking along the indicated line to obtain the individual microseparators.

Then a small drop of epoxy glue (Aradite Rapid ${ }^{\mathrm{TM}}$ ) was added to where the capillary exits the device. By turning the capillary and moving it in and out of the channel, the glue was driven in the capillary channel of the device. The glue must fill all the space between the capillary channel and the capillary itself, otherwise a dead volume is created at the connection (Figure 7.4). The estimated dead volume in a device where the glue was applied correctly was $0.06 \mu \mathrm{L}$, which fulfills the design requirement of $0.1 \mu \mathrm{L}$ or lower.

\subsection{Results and discussion}

\subsubsection{Nanowire formation}

After manufacturing the channels and micropillars in the silicon substrate, SEM analysis revealed a net of nanowires between the two channels of different depths (Figure 7.6). These wires are likely formed during the DRIE etching step with the buried mask. During DRIE etching, fluorocarbon is deposited with every iteration, and scallops in the order of several microns are formed. In the second DRIE step, the separation channel and the deeper capillary channel were etched. The nanowires are likely formed in this second etching step as a result of the scallops that were created in the first etching step (Figure 7.7).

These nanowires are undesirable for the intended gas/liquid separation, as they could block the bubbles from entering the device, locally increasing the pressure. Furthermore, the nanowires can break and resulting debris can obstruct the channel. By optimizing the DRIE procedure, smaller scallops and therefore more fragile wires can be formed. The thinner wires could be removed in an ultrasonic bath before bonding the silicon wafer with its glass cover. 


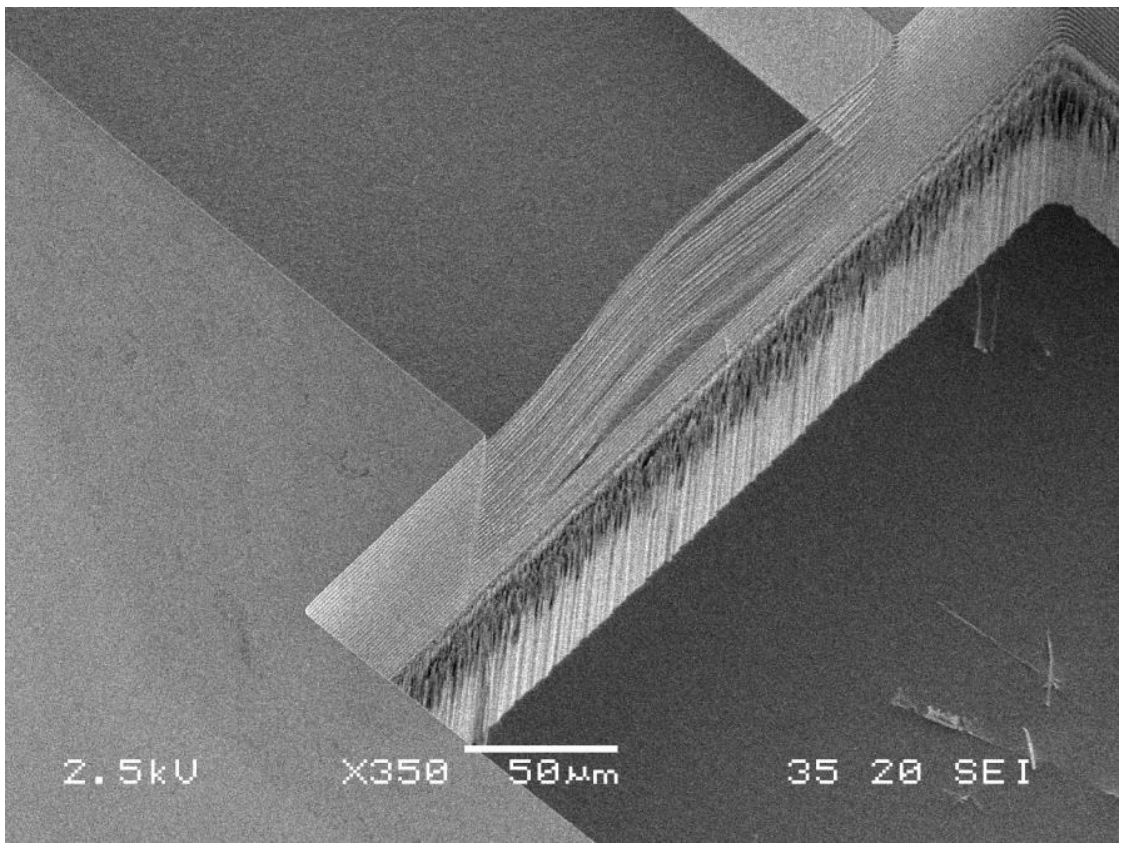

Figure 7.6 A net of nanowires was observed at the transition of the separation channel and the deeper capillary connection channel. SEM image.

A.

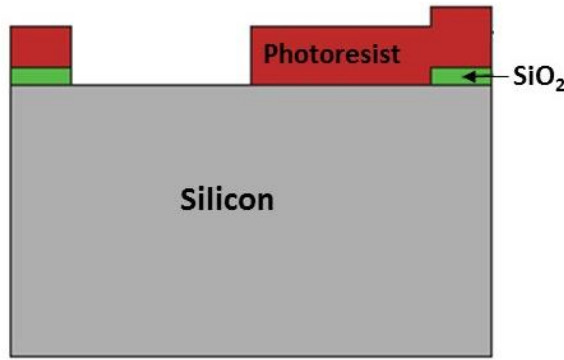

B.

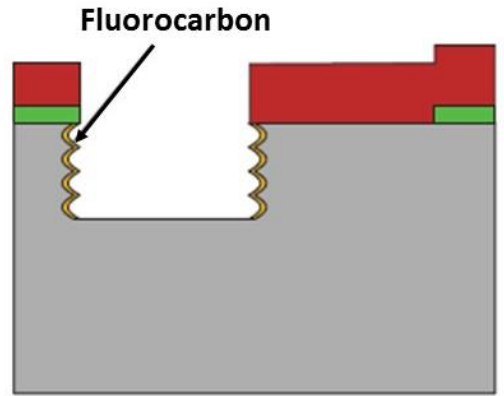

c.

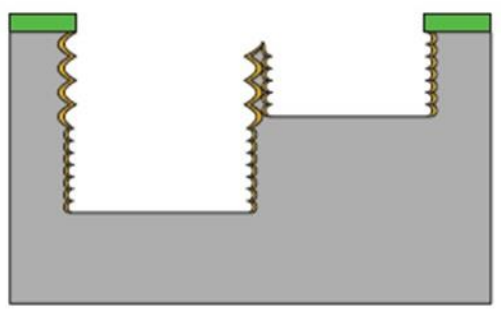

D.

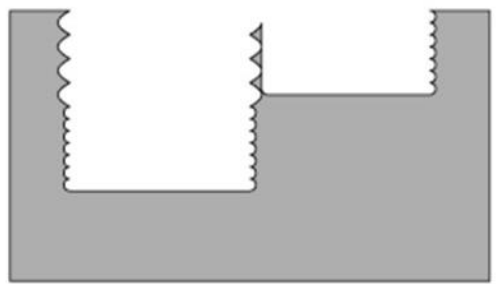

Figure 7.7 Formation of nanowires (schematic), with the photoresist in red, $\mathrm{SiO}_{2}$ in green, and fluorocarbon in yellow. First, the photoresist and the $\mathrm{SiO}_{2}$ hard mask are patterned according to design (A.), followed by DRIE etching of the deeper capillary channel (B.). After removal of the photoresist, a second etching step forms the separation channel and deepens the capillary channel (C.). The scallops formed in the dry-etching create the nanowires between the two channels (D.). 
Although the network of nanowires is undesired for phase separation, other applications would benefit greatly from such a nanonet. Silicon nanonets, as formed in this chapter, exhibit excellent transistor properties, which are interesting for sensing applications based on charge differences, such as $\mathrm{pH}^{24}$ and DNA hybridization. ${ }^{25}$ Nanonets can also act as traps, as was demonstrated with bacteria. ${ }^{26}$ The nanowire network can also be functionalized with for example noble metals for electrochemistry ${ }^{27}$ or biomolecules for fluorescent sensing. ${ }^{28}$ Nanowires exhibit higher thermal and electrical conductivity, ${ }^{27}$ compared to the conventional nanoparticles.

\subsubsection{Mechanical stability of the micropillars during fabrication}

The most fragile structures in the device are the micropillars (aspect ratio $H / P_{y}=20$ ), which are freestanding before the silicon substrate is bonded to glass. SEM analysis of the devices revealed negative tapering of the micropillars (Figure 7.8. A.), which has also been reported in the literature. ${ }^{29-31}$ Negative tapering is caused by the ratio of the etching ion, $\mathrm{SF}_{6}$, and the passivating ion, $\mathrm{C}_{4} \mathrm{~F}_{8}$ (fluorocarbon), which is in favor of the etching ion. ${ }^{30}$ During etching, the silicon substrate

A.

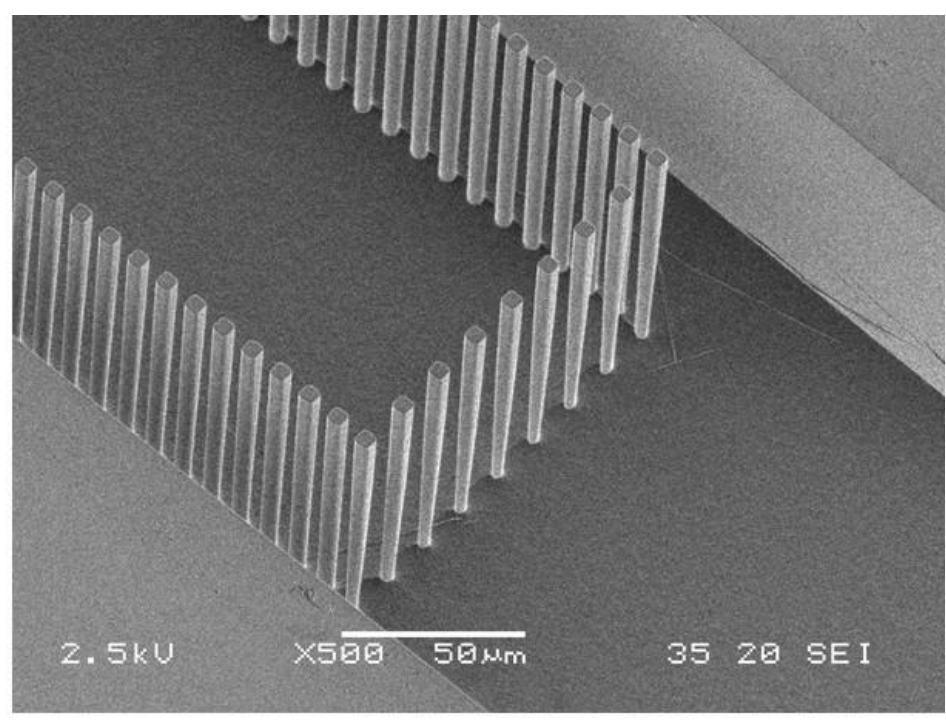

B.

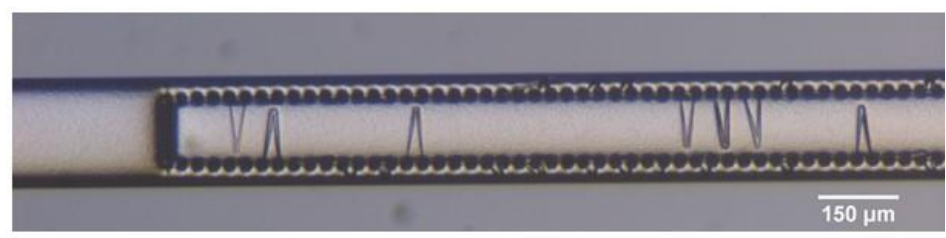

Figure 7.8 SEM image of tapered micropillars (A.) and optical microscope image of broken micropillars (top view, B.). 
is negatively charged, whereas the ions that etch the substrate are positively charged. As a result, the ion trajectory becomes bent, etching faster at the bottom of the micropillars. ${ }^{31-32}$ This negative tapering renders the micropillars more fragile, resulting in breaking of the pillars in some cases (Figure 7.8. B.).

\subsubsection{Mechanical stability of the micropillars: liquid pressure}

The mechanical stability of the micropillars was evaluated after bonding of the device. After connecting the microseparator to a syringe pump, MilliQ water containing a green food dye (2 vol\%) was flushed through the channel. The flow rate was gradually increased starting from 10

A.

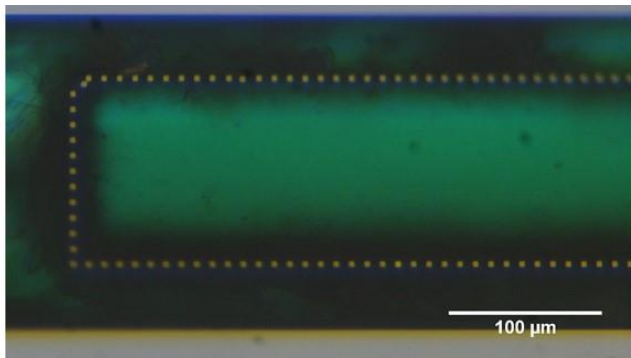

$1000 \mu \mathrm{LL} \cdot \mathrm{min}^{-1}$

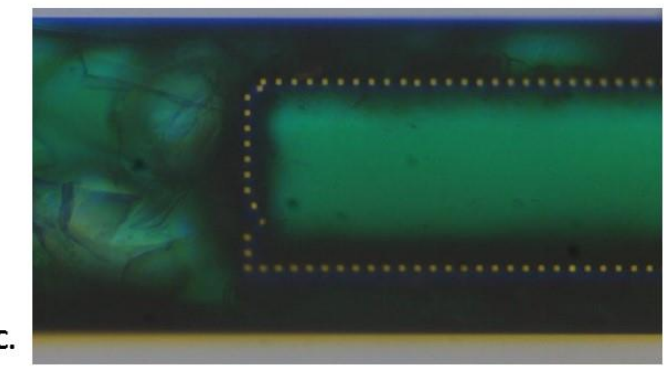

$1200 \mu \mathrm{L} \cdot \mathrm{min}^{-1}$

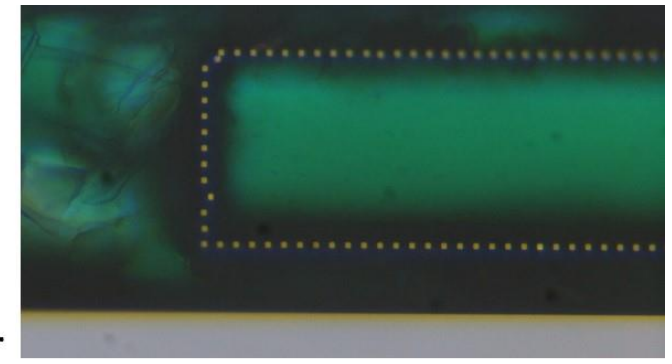

$1100 \mu \mathrm{L} . \mathrm{min}^{-1}$

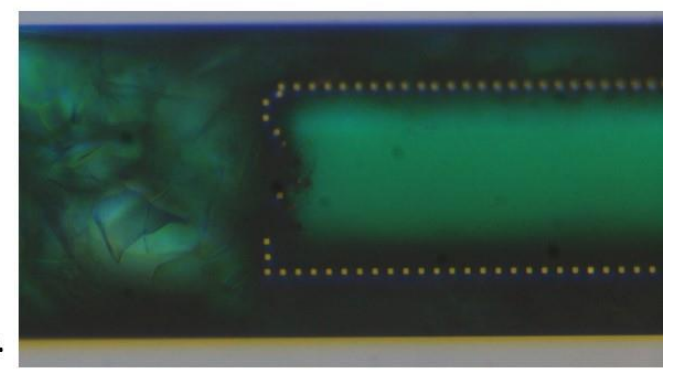

$1300 \mu \mathrm{L} . \mathrm{min}^{-1}$

Figure 7.9 Failure of the micropillar structures at increasing flow rate. First, the pillars at the corners are broken (A.), followed by the pillars in the center (B.-D.). Flow direction from left to right.

$\mu \mathrm{L} . \mathrm{min}^{-1}$ until the micropillars started to break at flow rates of $1000 \mu \mathrm{L} \cdot \mathrm{min}^{-1}$ and higher (Figure 7.9). The corner pillars of the micropillar membrane were the first to break under the fluid flow, as expected, as the shear stress is the highest at the solid boundaries of the device. This was followed by the collapse of the central pillars, which is consistent with a parabolic flow profile: the velocity and pressure in the liquid is highest at the centre of the channel. Overall, the device was able to operate at flow rates between 10 and $1000 \mu \mathrm{L} \cdot \mathrm{min}^{-1}$, fulfilling the initial requirements. 


\subsection{Conclusion}

In this chapter the fabrication of a modular gas/liquid microseparator based on a micropillar membrane is described. Here, liquid can flow through the micropillar membrane, whereas gas bubbles would not permeate as a result of their surface tension. Pillars with a high aspect ratio of 20 could withstand flow rates up to $1000 \mu \mathrm{L} \cdot \mathrm{min}^{-1}$. The in- and outlets consisted of capillaries glued into the device in the same plane as the channel, which not only reduced the dead volume to $0.06 \mu \mathrm{L}$, but also prevented flow disturbances.

\section{Acknowledgements}

I would like to thank Constantijn Rip for his valuable contribution to this project during his bachelor assignment.

\section{References}

1. Cortright, R. D.; Davda, R. R.; Dumesic, J. A., Hydrogen from catalytic reforming of biomass-derived hydrocarbons in liquid water. Nature 2002, 418 (6901), 964-967.

2. Davda, R. R.; Shabaker, J. W.; Huber, G. W.; Cortright, R. D.; Dumesic, J. A., A review of catalytic issues and process conditions for renewable hydrogen and alkanes by aqueous-phase reforming of oxygenated hydrocarbons over supported metal catalysts. Applied Catalysis BEnvironmental 2005, 56 (1-2), 171-186.

3. Chheda, J. N.; Huber, G. W.; Dumesic, J. A., Liquid-phase catalytic processing of biomassderived oxygenated hydrocarbons to fuels and chemicals. Angewandte Chemie-International Edition 2007, 46 (38), 7164-7183.

4. Kirilin, A. V.; Tokarev, A. V.; Kustov, L. M.; Salmi, T.; Mikkola, J. P.; Murzin, D. Y., Aqueous phase reforming of xylitol and sorbitol: Comparison and influence of substrate structure. Applied Catalysis a-General 2012, 435, 172-180.

5. Luo, N. J.; Fu, X. W.; Cao, F. H.; Xiao, T. C.; Edwards, P. P., Glycerol aqueous phase reforming for hydrogen generation over Pt catalyst - Effect of catalyst composition and reaction conditions. Fuel 2008, 87 (17-18), 3483-3489. 
6. Wilkinson, D.; Waldie, B.; Mohamad Nor, M. I.; Yen Lee, H., Baffle plate configurations to enhance separation in horizontal primary separators. Chem. Eng. J. 2000, 77 (3), 221-226.

7. Chabanon, E.; Belaissaoui, B.; Favre, E., Gas-liquid separation processes based on physical solvents: opportunities for membranes. Journal of Membrane Science 2014, 459, 52-61. 8. Rosa, E. S.; França, F. A.; Ribeiro, G. S., The cyclone gas-liquid separator: operation and mechanistic modeling. Journal of Petroleum Science and Engineering 2001, 32 (2), 87-101.

9. Temiz, Y.; Lovchik, R. D.; Kaigala, G. V.; Delamarche, E., Lab-on-a-chip devices: How to close and plug the lab? Microelectron. Eng. 2015, 132, 156-175.

10. Liu, D.; Zhang, H.; Fontana, F.; Hirvonen, J. T.; Santos, H. A., Current developments and applications of microfluidic technology toward clinical translation of nanomedicines. Advanced Drug Delivery Reviews 2018, 128, 54-83.

11. Zhang, J.; Yan, S.; Yuan, D.; Alici, G.; Nguyen, N. T.; Warkiani, M. E.; Li, W. H., Fundamentals and applications of inertial microfluidics: a review. Lab on a Chip 2016, 16 (1), 1034.

12. Reizman, B. J.; Jensen, K. F., Feedback in Flow for Accelerated Reaction Development. Acc. Chem. Res. 2016, 49 (9), 1786-1796.

13. Grant, J.; Goudarzi, S. H.; Mrksich, M., High-Throughput Enzyme Kinetics with 3D Microfluidics and Imaging SAMDI Mass Spectrometry. Anal. Chem. 2018, 90 (21), 13096-13103. 14. Bings, N. H.; Wang, C.; Skinner, C. D.; Colyer, C. L.; Thibault, P.; Harrison, D. J., Microfluidic Devices Connected to Fused-Silica Capillaries with Minimal Dead Volume. Anal. Chem. 1999, 71 (15), 3292-3296.

15. Li, J.; Thibault, P.; Bings, N. H.; Skinner, C. D.; Wang, C.; Colyer, C.; Harrison, J., Integration of Microfabricated Devices to Capillary Electrophoresis-Electrospray Mass Spectrometry Using a Low Dead Volume Connection: Application to Rapid Analyses of Proteolytic Digests. Anal. Chem. 1999, 71 (15), 3036-3045.

16. $\mathrm{Xu}, \mathrm{J}$;; Vaillant, R.; Attinger, D., Use of a porous membrane for gas bubble removal in microfluidic channels: physical mechanisms and design criteria. Microfluidics and Nanofluidics 2010, 9 (4-5), 765-772.

17. Lochovsky, C.; Yasotharan, S.; Gunther, A., Bubbles no more: in-plane trapping and removal of bubbles in microfluidic devices. Lab on a Chip 2012, 12 (3), 595-601. 
18. Chen, H. X.; Xu, J. L.; Yan, Y. Y.; Zhou, W. N., Phase separation and flow pattern modulation with a T-type micro-drainage system. Appl. Therm. Eng. 2017, 122, 214-226.

19. Zenith, F.; Kraus, M.; Krewer, U., Model-based analysis of micro-separators for portable direct methanol fuel-cell systems. Computers \& Chemical Engineering 2012, 38, 64-73.

20. Roydhouse, M. D.; Pradas, M.; Al-Rifai, N.; Azizi, B.; Cao, E. H.; Kalliadasis, S.; Gavriilidis, A., Operating ranges of gas-liquid capillary microseparators: Experiments and theory. Chem. Eng. Sci. 2014, 114, 30-39.

21. Xu, J. L.; An, B.; Sun, D. L., The phase separation in a rectangular microchannel by micromembrane. Appl. Therm. Eng. 2015, 88, 172-184.

22. An, B.; Xu, J. L., Investigation on a micro-pin-fin based membrane separator. Int. J. Heat Mass Transfer 2016, 95, 426-439.

23. Tiggelaar, R. M.; Benito-Lopez, F.; Hermes, D. C.; Rathgen, H.; Egberink, R. J. M.; Mugele, F. G.; Reinhoudt, D. N.; van den Berg, A.; Verboom, W.; Gardeniers, H., Fabrication, mechanical testing and application of high-pressure glass microreactor chips. Chem. Eng. J. 2007, $131(1-3), 163-170$.

24. Jin, B.; Lee, G.-Y.; Park, C.; Kim, D.; Choi, W.; Yoo, J.-W.; Pyun, J.-C.; Lee, J.-S., Electrical Characteristics and pH Response of a Parylene-H Sensing Membrane in a Si-Nanonet Ion-Sensitive Field-Effect Transistor. Sensors (Basel, Switzerland) 2018, 18 (11), 3892.

25. Nguyen, T. T. T.; Legallais, M.; Morisot, F.; Cazimajou, T.; Stambouli, V.; Mouis, M.; Salem, B.; Ternon, C., First evidence of superiority of Si nanonet field effect transistors over multiparallel Si nanowire ones in view of electrical DNA hybridization detection. Materials Research Express 2019, 6 (1).

26. De Cesare, F.; Di Mattia, E.; Macagnano, A., Fishing bacteria with a nanonet. Mater. Today 2017, 20 (5), 284-285.

27. Liu, R.; Yu, X.; Zhang, G.; Zhang, S.; Cao, H.; Dolbecq, A.; Mialane, P.; Keita, B.; Zhi,

L., Polyoxometalate-mediated green synthesis of a 2D silver nanonet/graphene nanohybrid as a synergistic catalyst for the oxygen reduction reaction. Journal of Materials Chemistry A 2013, 1 (38), 11961-11969.

28. Kim, T.; Park, J.; Jin, H. J.; Lee, H.; Byun, K.-E.; Lee, C.-S.; Kim, K. S.; Hong, B. H.; Kim, T. H.; Hong, S., Graphene nanonet for biological sensing applications. Nanotechnology 2013, 24 (37), 375302. 
29. Grigoras, K.; Sainiemi, L.; Tiilikainen, J.; Säynätjoki, A.; Airaksinen, V. M.; Franssila, S., Application of ultra-thin aluminum oxide etch mask made by atomic layer deposition technique. Journal of Physics: Conference Series 2007, 61, 369-373.

30. Ayari-Kanoun, A.; Aydinoglu, F.; Cui, B.; Saffih, F., Silicon nanostructures with very large negatively tapered profile by inductively coupled plasma-RIE. Journal of Vacuum Science \& Technology B 2016, 34 (6), 06KD01.

31. Boer, M. J. d.; Gardeniers, J. G. E.; Jansen, H. V.; Smulders, E.; Gilde, M.; Roelofs, G.; Sasserath, J. N.; Elwenspoek, M., Guidelines for etching silicon MEMS structures using fluorine high-density plasmas at cryogenic temperatures. Journal of Microelectromechanical Systems 2002, $11(4), 385-401$.

32. Jansen, H.; Boer, M. d.; Legtenberg, R.; Elwenspoek, M., The black silicon method: a universal method for determining the parameter setting of a fluorine-based reactive ion etcher in deep silicon trench etching with profile control. Journal of Micromechanics and Microengineering 1995, 5 (2), 115-120. 


\section{A7.1 Process flow}

\section{ILP: In-line Processing}

\begin{tabular}{|l|l|}
\hline MIP: Metal-free & UCP: Ultra Clean \\
Processing & Processing
\end{tabular}

Step Level Process/Basic flow

Substrate Silicon

(\#subs 102)
NL-CLR-Wafer Storage Cupboard

Orientation: <100>

Diameter: $100 \mathrm{~mm}$

Thickness: $525 \mu \mathrm{m}+/-25 \mu \mathrm{m}$

Polished: Double side (DSP)

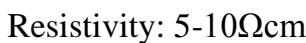

Type: p/boron

\section{film1940: Wet Oxidation of Silicon (B2)}

2

3

4

$\begin{array}{ll}\text { MFP } & \text { Quick Dump Rinse } \\ \text { (QDR) }\end{array}$

(\#rinse002)

5

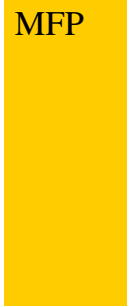

Cleaning in $69 \%$

$\mathrm{HNO}_{3}\left(95{ }^{\circ} \mathrm{C}\right)$

(\#clean003)

\section{Cleaning in $99 \% \mathrm{HNO}_{3}$ NL-CLR-WB14}

(\#clean001)

Purpose: removal of organic traces.

- Beaker 1: $99 \% \mathrm{HNO}_{3}$

- Time $=5 \mathrm{~min}$

\section{Cleaning in 99\% $\mathrm{HNO}_{3}$ NL-CLR-WB14}

(\#clean002)

Purpose: removal of organic traces.

- Beaker 2: $99 \% \mathrm{HNO}_{3}$

- Time $=5 \mathrm{~min}$

\section{NL-CLR-Wetbenches}

Purpose: removal of traces of chemical agents.

Recipe 1 Quick dump rinsing (QDR)

Recipe 2 Cascade rinsing for fragile wafers

Rinse until message 'End of rinsing process' is shown on the touchscreen of the QDR, else repeat the rinsing process.

\section{NL-CR-WB14}

Purpose: removal of metallic traces.

- Beaker 3A or 3B: $69 \% \mathrm{HNO}_{3}$

- Temperature $=95^{\circ} \mathrm{C}$

- Time $=10 \mathrm{~min}$ 
6

7

8

MFP Etching in 1\% HF
(\#etch127)

9

\section{MFP}

Quick Dump Rinse (QDR)

(\#rinse002)

Substrate drying

(WB14)

(\#dry022)
Quick Dump Rinse

(QDR)

(\#rinse002)

\section{NL-CLR-Wetbenches}

Purpose: removal of traces of chemical agents.

Recipe 1 Quick dump rinsing (QDR)

Recipe 2 Cascade rinsing for fragile wafers

Rinse until message 'End of rinsing process' is shown on the touchscreen of the QDR, else repeat the rinsing process.

\section{NL-CLR-WB14}

Optional drying step. After the QDR, you can transfer your substrates directly to a Teflon carrier and strip the native $\mathrm{SiO} 2$ in $1 \% \mathrm{HF}$ (WB15).

\section{Single substrate drying:}

1. Use the single-wafer spinner

Settings: $2500 \mathrm{rpm}, 60 \mathrm{sec}$ (including $45 \mathrm{sec}$ nitrogen purge)

2. Use the nitrogen gun (fragile wafers or small samples)

\section{Batch drying of substrates:}

The Semitool uses the following standard procedure:

- Rinse: $30 \mathrm{sec}(600 \mathrm{rpm})$

- Q-rinse: $10.0 \mathrm{M} \Omega$ (600 rpm)

- Purge: $10 \mathrm{sec}$ (600 rpm)

- Drying: $280 \mathrm{sec}(1600 \mathrm{rpm})$

Note: it is obligatory to apply a single rinsing step in the QDR before using the Semitool!

\section{NL-CLR-WB15}

Purpose: remove native $\mathrm{SiO} 2$ from silicon.

Beaker: $1 \% \mathrm{HF}$

Temperature: room temperature

Time $=1 \mathrm{~min}$

This step is obligatory for the MESA+ monitor wafer (if applicable, see Equipment database).

\section{NL-CLR-Wetbenches}

Purpose: removal of traces of chemical agents.

Recipe 1 Quick dump rinsing (QDR)

Recipe 2 Cascade rinsing for fragile wafers

Rinse until message 'End of rinsing process' is shown on the touchscreen of the QDR, else repeat the rinsing process. 


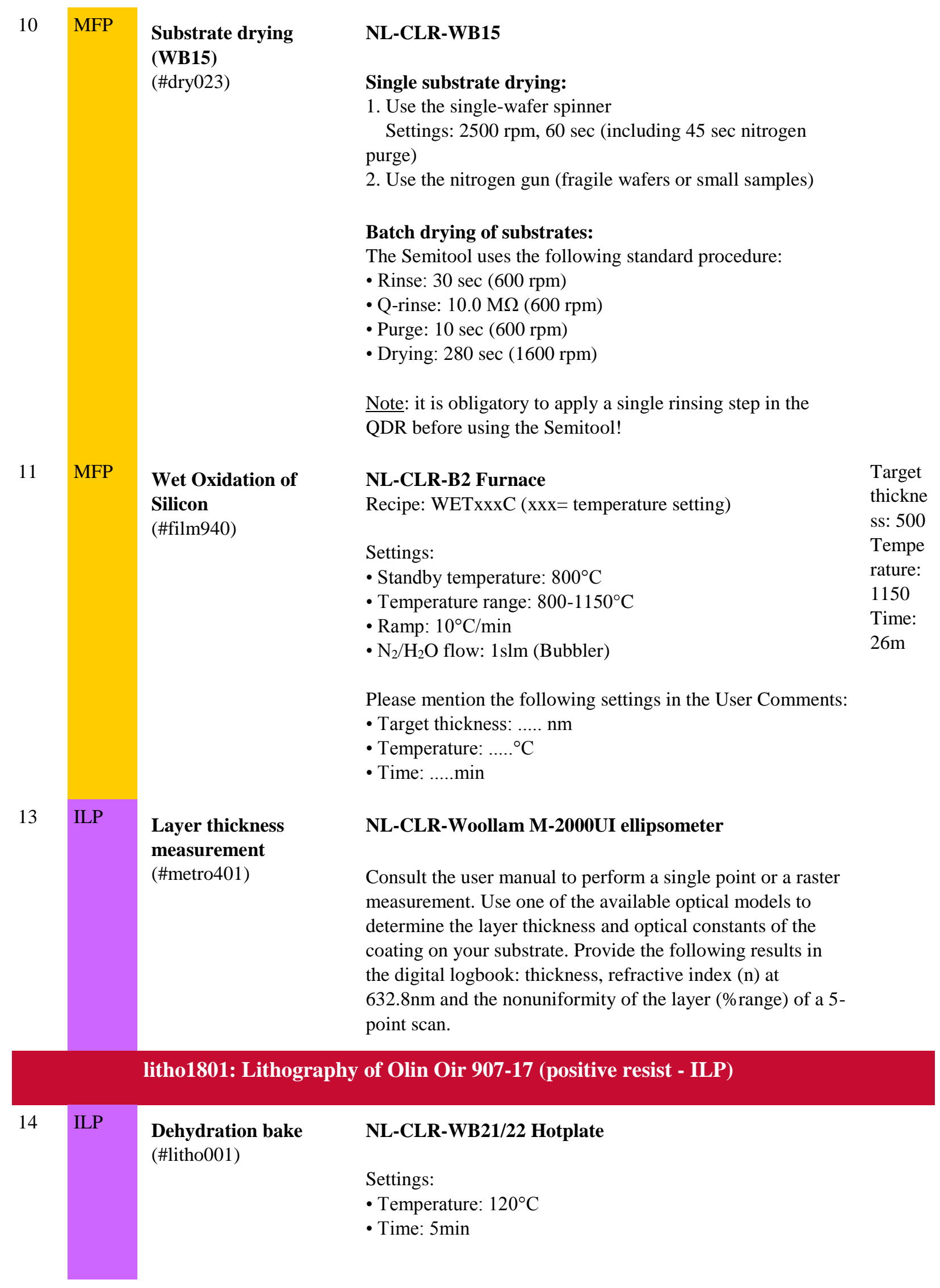




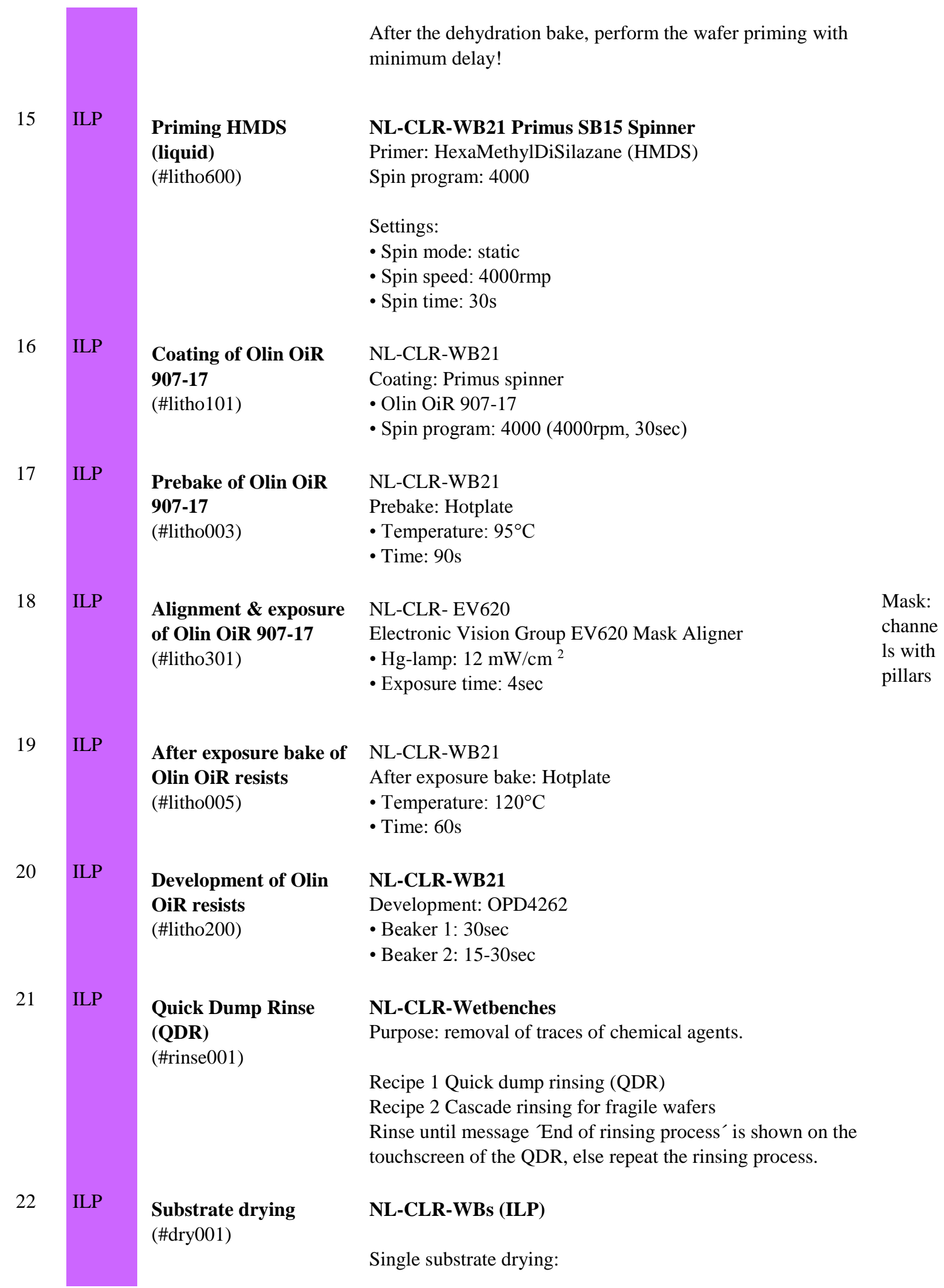




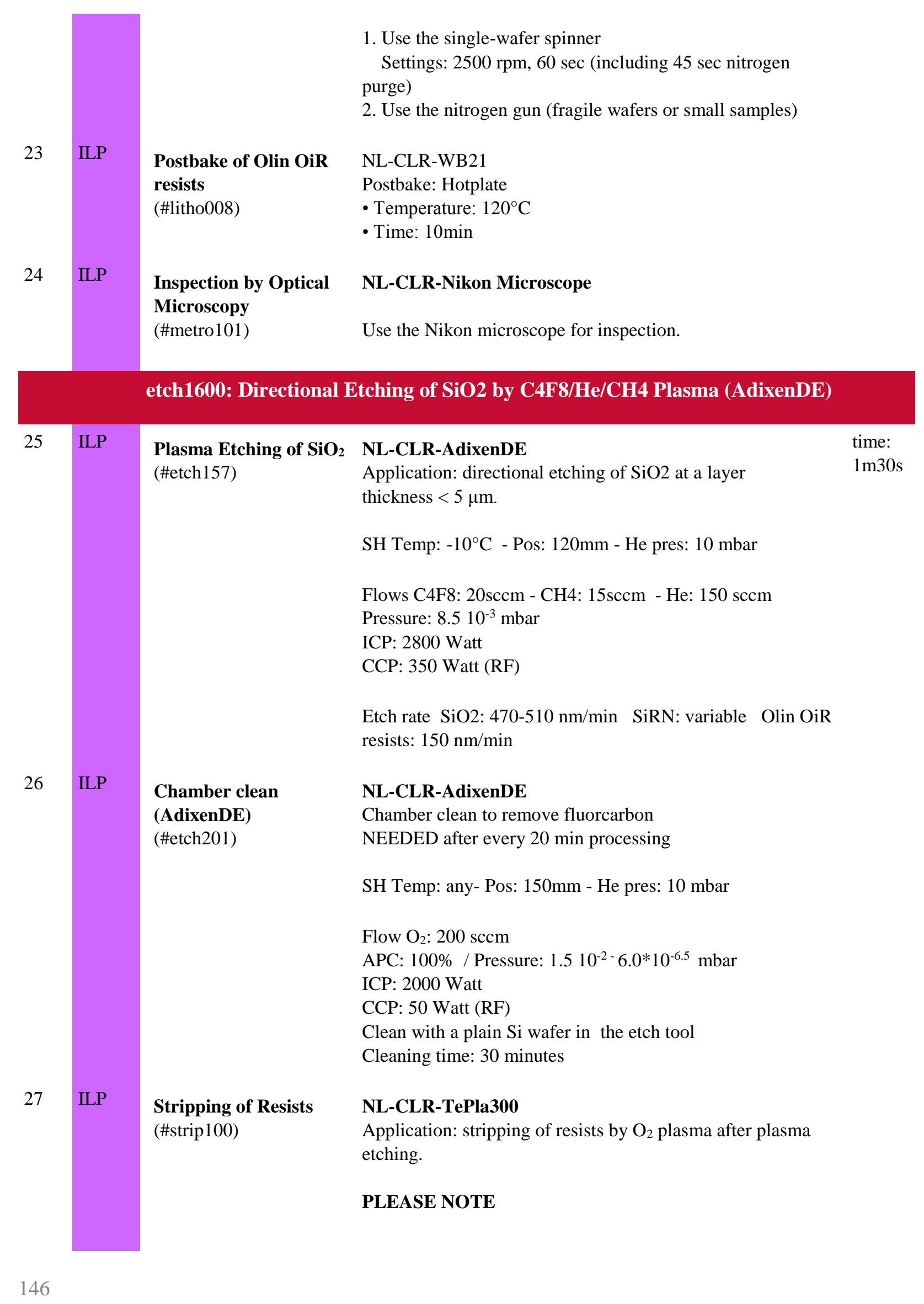


1. RESTRICTION: do not strip resists on chromium in the TePla300, but instead use the TePla360 (choose: recipe 041). 2. BACKUP: TePla300 down? Contact the administrator if you can continue your processing in the TePla360.

\begin{tabular}{|l|c|c|c}
\hline Step & $\begin{array}{c}\mathbf{O}_{2} \\
(\mathbf{s c c m})\end{array}$ & $\begin{array}{c}\mathbf{N}_{2} \\
(\mathbf{s c c m})\end{array}$ & $\begin{array}{c}\mathbf{P} \\
(\mathbf{m b a r})\end{array}$ \\
\hline Preheating & 0 & 500 & 1.0 \\
\hline Stripping of resist & 500 & 0 & 1.0 \\
\hline
\end{tabular}

* Select one of the following recipes to strip the resist, depending on the thickness of the resist, treatment of the resist and the number of wafers. Use the abort option in the last step if you sample requires a shorter stripping time.

Recipe 01: time $=10 \mathrm{~min}$

Recipe 02: time $=30 \mathrm{~min}$

Recipe 04: time $=60 \mathrm{~min}$

\section{NL-CLR-WB09}

Purpose: removal of metal traces originating from plasma tools in order to protect the cleaning efficiency of the wet benches. For this reason, RCA-2 is compulsory in case you continue:

- cleaning in the Pre-Furnace Clean (WB14-MFP)

- processing in the Ultra-Clean Line - Front End (WB12-

$\mathrm{UCP})$

- processing in the Ultra-Clean Line - Back End (WB13$\mathrm{UCP})$

Chemicals: $\mathrm{HCl}: \mathrm{H}_{2} \mathrm{O}_{2}: \mathrm{H}_{2} \mathrm{O}$ (1:1:5 vol.\%)

\section{PLEASE NOTE}

1. CAUTION: do not process substrates with metal patterns in RCA-2.

2. NO REUSE: reuse of RCA- 2 is forbidden! Contact the administrator in case there is no empty RCA- 2 beaker available in WB09.

Procedure:

- Pour $1500 \mathrm{ml} *$ of DI water into the beaker

- Turn on the stirrer

- Add 300ml* of Hydrogen Chloride ( $\mathrm{HCl})$

- Heat up the solution to $70^{\circ} \mathrm{C}$ (setpoint heater $=80^{\circ} \mathrm{C}$ )

- Slowly add $300 \mathrm{ml} *$ of Hydrogen Peroxide $\left(\mathrm{H}_{2} \mathrm{O}_{2}\right)$ 
29

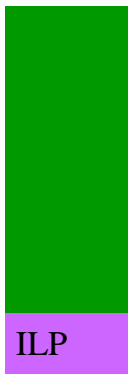

Quick Dump Rinse (QDR)

(\#rinse001)

30

ILP

Substrate drying

(\#dry001)
- Submerge your samples as soon as the temperature is above $70^{\circ} \mathrm{C}$

- Time $=15 \mathrm{~min}$

* Use a glass graduated cylinder of $500 \mathrm{ml}$ to measure the volume of the chemicals.

\section{NL-CLR-Wetbenches}

Purpose: removal of traces of chemical agents.

Recipe 1 Quick dump rinsing (QDR)

Recipe 2 Cascade rinsing for fragile wafers

Rinse until message 'End of rinsing process' is shown on the touchscreen of the QDR, else repeat the rinsing process.

\section{NL-CLR-WBs (ILP)}

Single substrate drying:

1. Use the single-wafer spinner

Settings: $2500 \mathrm{rpm}, 60 \mathrm{sec}$ (including $45 \mathrm{sec}$ nitrogen purge)

2. Use the nitrogen gun (fragile wafers or small samples)

\section{litho1801: Lithography of Olin Oir 907-17 (positive resist - ILP)}

\section{$31 \quad$ ILP}

32

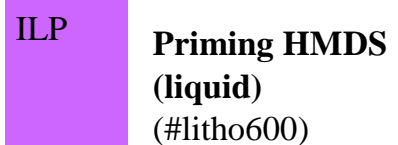

33
Dehydration bake (\#litho001)

(\#litho600)

Coating of Olin OiR

907-17

(\#litho101)

\section{NL-CLR-WB21/22 Hotplate}

Settings:

- Temperature: $120^{\circ} \mathrm{C}$

- Time: $5 \mathrm{~min}$

After the dehydration bake, perform the wafer priming with minimum delay!

\section{NL-CLR-WB21 Primus SB15 Spinner}

Primer: HexaMethylDiSilazane (HMDS)

Spin program: 4000

Settings:

- Spin mode: static

- Spin speed: 4000rpm

- Spin time: $30 \mathrm{~s}$

NL-CLR-WB21

Coating: Primus spinner

- Olin OiR 907-17

- Spin program: 4000 (4000rpm, 30sec) 


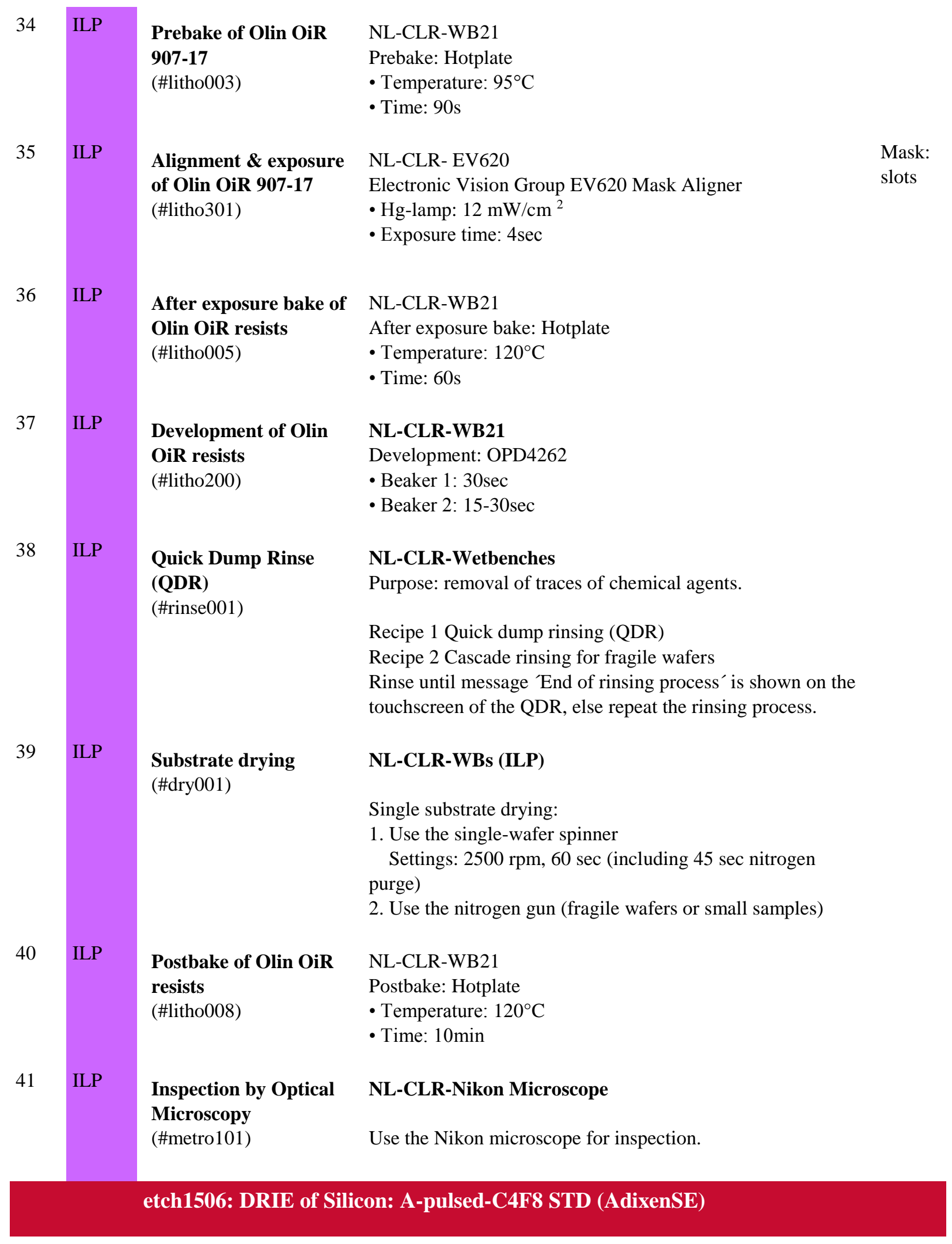


DRIE of Si A pulsed $\mathrm{C} 4 \mathrm{F8}$ at $-\mathbf{4 0}^{\circ} \mathrm{C}$ (\#etch175)
NL-CLR-Adixen SE

Application: trenches, wafer through using thick photoresist (908-35)

Use: C4F8 flow and CCP for tuning process.

SH temp: $-40^{\circ} \mathrm{C}-$ Pos: $110 \mathrm{~mm}-\mathrm{He}$ pres: $10 \mathrm{mbar}$

\begin{tabular}{|c|c|c|c|c|c|}
\hline \multicolumn{2}{|c|}{ Parameters } & \multicolumn{2}{|c|}{ Etch } & \multicolumn{2}{|c|}{ Deposition } \\
\hline \multicolumn{2}{|l|}{ Gas } & \multicolumn{2}{|r|}{$\mathrm{SF}_{6}$} & \multicolumn{2}{|r|}{$\mathrm{C}_{4} \mathrm{~F}_{8}$} \\
\hline \multicolumn{2}{|c|}{ Flow (sccm) } & \multicolumn{2}{|r|}{500} & \multicolumn{2}{|r|}{175} \\
\hline \multicolumn{2}{|c|}{ Time (sec) } & \multicolumn{2}{|r|}{4} & \multicolumn{2}{|r|}{0.5} \\
\hline \multicolumn{2}{|l|}{ Priority } & \multicolumn{2}{|r|}{2} & \multicolumn{2}{|r|}{1} \\
\hline \multicolumn{2}{|l|}{ APC \% } & \multicolumn{2}{|r|}{15} & \multicolumn{2}{|r|}{15} \\
\hline \multicolumn{2}{|c|}{ ICP (Watt) } & \multicolumn{2}{|r|}{2500} & \multicolumn{2}{|r|}{2500} \\
\hline \multicolumn{2}{|c|}{ CCP (Watt) } & \multicolumn{2}{|r|}{20} & \multicolumn{2}{|r|}{20} \\
\hline \multicolumn{2}{|c|}{ Pulsed (msec) } & \multicolumn{2}{|c|}{ 20on/80off } & \multicolumn{2}{|c|}{ 20on/80off } \\
\hline $\begin{array}{l}\mathrm{CCP} \\
{[\mathbf{w}]}\end{array}$ & \multicolumn{2}{|c|}{$\begin{array}{c}\text { On/off }[\mathrm{m} \\
\text { sec }]\end{array}$} & $\begin{array}{c}\text { C4F8 } \\
\text { [sscm] }\end{array}$ & $\begin{array}{l}\text { Er resist } \\
\text { nm/min }\end{array}$ & $\begin{array}{c}\text { silicon } \\
\{\text { um/min }]\end{array}$ \\
\hline 20 & \multicolumn{2}{|c|}{$20 / 180$} & 20 & $33-50$ & 10 \\
\hline 20 & \multicolumn{2}{|c|}{$35 / 165$} & 25 & 80 & 10 \\
\hline
\end{tabular}

ILP Cleaning of chamber (\#etch202)

\section{NL-CLR-AdixenSE}

Purpose: cleaning of the chamber by removal of fluorcarbon residues

Application: post-processing after etching your substrates with fluorocarbon plasmas

SH temperature: any temperature

Pos: 200mm

He pres: $10 \mathrm{mbar}$

\begin{tabular}{|l|l|}
\hline Parameters & Value \\
\hline Gas & $\mathrm{O}_{2}$ \\
\hline Flow (sccm) & 200 \\
\hline APC \% & 100 \\
\hline ICP (Watt) & 2000 \\
\hline CCP (Watt) & 50 RF (SH gen 1) \\
\hline Wafer & Si dummy wafer \\
\hline
\end{tabular}

depth:

$100 \mu \mathrm{m}$ , time:

$5 \mathrm{~min}$ 


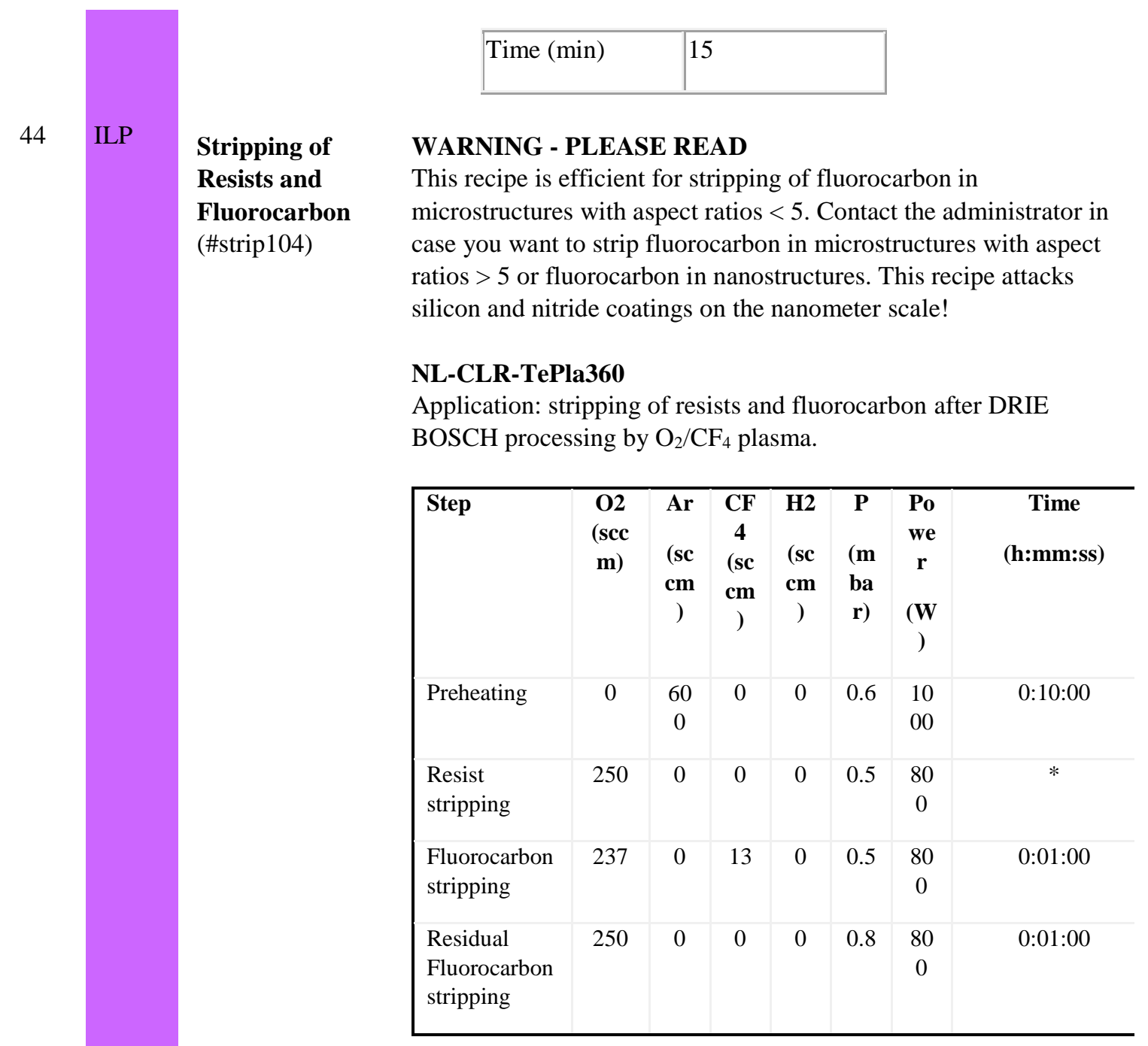

* Select one of the following recipes depending on the thickness of the resist, treatment of the resist and the number of wafers.

Recipe 035: time $=10 \mathrm{~min}$

Recipe 037: time $=20 \mathrm{~min}$

Recipe 036: time $=60 \mathrm{~min}$

BACKUP: The TePla300 is not a backup for this processing! If the TePla360 is down, contact the administrator.

PLEASE NOTE It is mandatory to remove metal traces originating from plasma tools in RCA-2 (residue1505), e.g. plasma etching or stripping in $\mathrm{O}_{2}$ plasma, in case you:

- continue with UCP processing

- continue with high-temperature processing (MFP) 


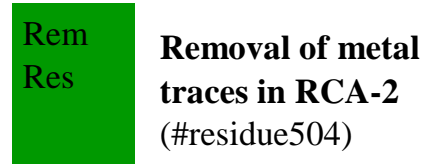

46

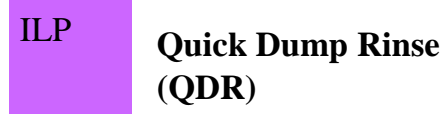

47

\section{traces in RCA-2}

(\#residue504) (\#rinse001)

\section{NL-CLR-WB09}

Purpose: removal of metal traces originating from plasma tools in order to protect the cleaning efficiency of the wet benches. For this reason, RCA-2 is compulsory in case you continue:

- cleaning in the Pre-Furnace Clean (WB14-MFP)

- processing in the Ultra-Clean Line - Front End (WB12-

$\mathrm{UCP})$

- processing in the Ultra-Clean Line - Back End (WB13$\mathrm{UCP})$

Chemicals: $\mathrm{HCl}: \mathrm{H}_{2} \mathrm{O}_{2}: \mathrm{H}_{2} \mathrm{O}(1: 1: 5$ vol.\%)

\section{PLEASE NOTE}

1. CAUTION: do not process substrates with metal patterns in RCA-2.

2. NO REUSE: reuse of RCA- 2 is forbidden! Contact the administrator in case there is no empty RCA- 2 beaker available in WB09.

Procedure:

- Pour $1500 \mathrm{ml} *$ of DI water into the beaker

- Turn on the stirrer

- Add 300ml* of Hydrogen Chloride ( $\mathrm{HCl})$

- Heat up the solution to $70^{\circ} \mathrm{C}$ (setpoint heater $=80^{\circ} \mathrm{C}$ )

- Slowly add $300 \mathrm{ml}^{*}$ of Hydrogen Peroxide $\left(\mathrm{H}_{2} \mathrm{O}_{2}\right)$

- Submerge your samples as soon as the temperature is above $70^{\circ} \mathrm{C}$

- Time $=15 \mathrm{~min}$

* Use a glass graduated cylinder of $500 \mathrm{ml}$ to measure the volume of the chemicals.

\section{NL-CLR-Wetbenches}

Purpose: removal of traces of chemical agents.

Recipe 1 Quick dump rinsing (QDR)

Recipe 2 Cascade rinsing for fragile wafers

Rinse until message 'End of rinsing process' is shown on the touchscreen of the QDR, else repeat the rinsing process.

\section{NL-CLR-WBs (ILP)}

\section{(\#dry001)}

Single substrate drying:

1. Use the single-wafer spinner

Settings: $2500 \mathrm{rpm}, 60 \mathrm{sec}$ (including $45 \mathrm{sec}$ nitrogen purge)

2. Use the nitrogen gun (fragile wafers or small samples) 
etch1652: DRIE of silicon: Bosch HARS (SPTS Pegasus)

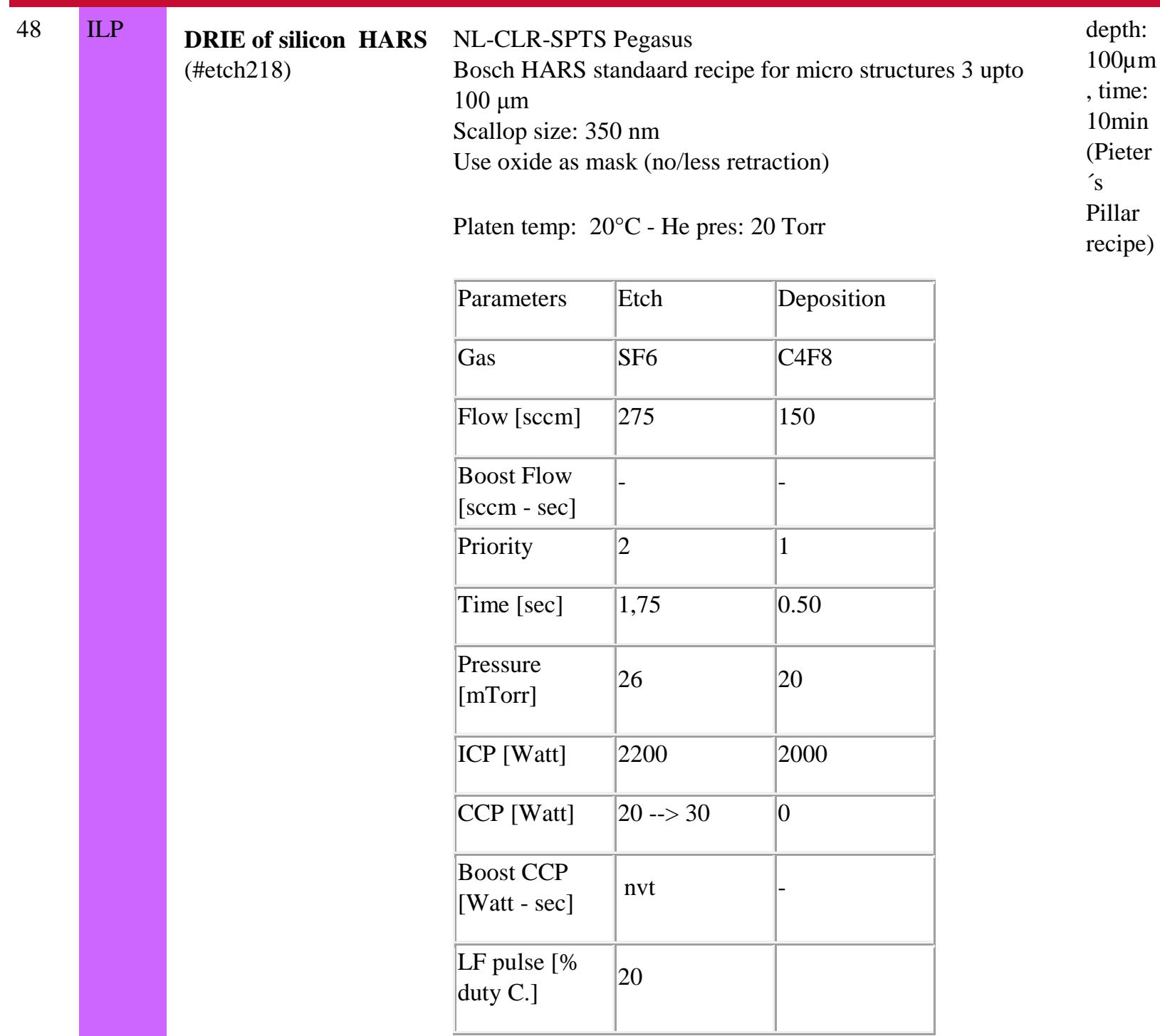

Etch rate $\mathrm{Si}: 10 \mu \mathrm{m} / \mathrm{min}$, Olin907: $80 \mathrm{~nm} / \mathrm{min}$

49

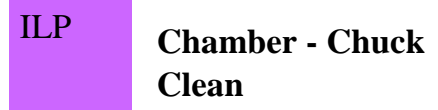

(\#etch219)

\section{NL-CLR-SPTS Pegasus}

Application: removal of organic and fluorocarbon residues from the chuck and chamber wall.

Recipe: Clean after use

Parameters:

$\mathrm{O}_{2}$ flow: $200 \mathrm{sccm}$

Pressure: $5 \mathrm{mTorr}$

ICP: $2500 \mathrm{~W}$

CCP: 20W (RF) 


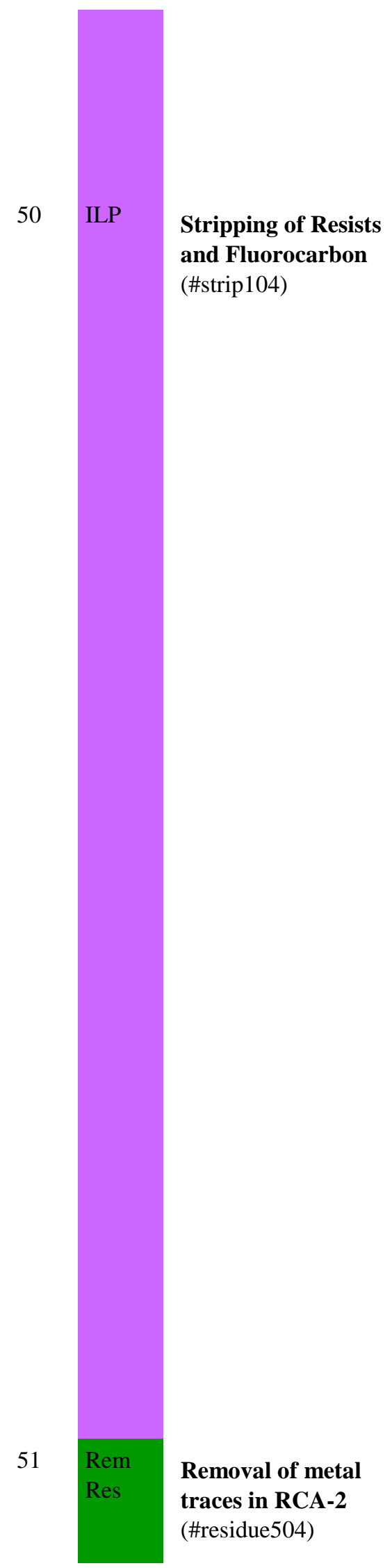

Time: $15 \mathrm{~min}$

Note: after cleaning the substrate holder temp will be set to $20^{\circ} \mathrm{C}$. Perform this recipe at the moment you are done etching.

\section{WARNING - PLEASE READ}

This recipe is efficient for stripping of fluorocarbon in microstructures with aspect ratios $<5$. Contact the administrator in case you want to strip fluorocarbon in microstructures with aspect ratios $>5$ or fluorocarbon in nanostructures. This recipe attacks silicon and nitride coatings on the nanometer scale!

\section{NL-CLR-TePla360}

Application: stripping of resists and fluorocarbon after DRIE $\mathrm{BOSCH}$ processing by $\mathrm{O}_{2} / \mathrm{CF}_{4}$ plasma.

\begin{tabular}{|lcc|}
\hline Step & $\begin{array}{c}\text { O2 } \\
\text { (sccm) }\end{array}$ & $\begin{array}{c}\text { Ar } \\
\text { (sccm) }\end{array}$ \\
Preheating & 0 & 600 \\
Resist stripping & 250 & 0 \\
Fluorocarbon stripping & 237 & 0 \\
Residual Fluorocarbon stripping & 250 & 0 \\
\hline
\end{tabular}

* Select one of the following recipes depending on the thickness of the resist, treatment of the resist and the number of wafers.

Recipe 035: time $=10 \mathrm{~min}$

Recipe 037: time $=20 \mathrm{~min}$

Recipe 036: time $=60 \mathrm{~min}$

BACKUP: The TePla300 is not a backup for this processing! If the TePla360 is down, contact the administrator.

PLEASE NOTE It is mandatory to remove metal traces originating from plasma tools in RCA-2 (residue1505), e.g. plasma etching or stripping in $\mathrm{O}_{2}$ plasma, in case you:

- continue with UCP processing

- continue with high-temperature processing (MFP)

\section{NL-CLR-WB09}

Purpose: removal of metal traces originating from plasma tools in order to protect the cleaning efficiency of the wet 


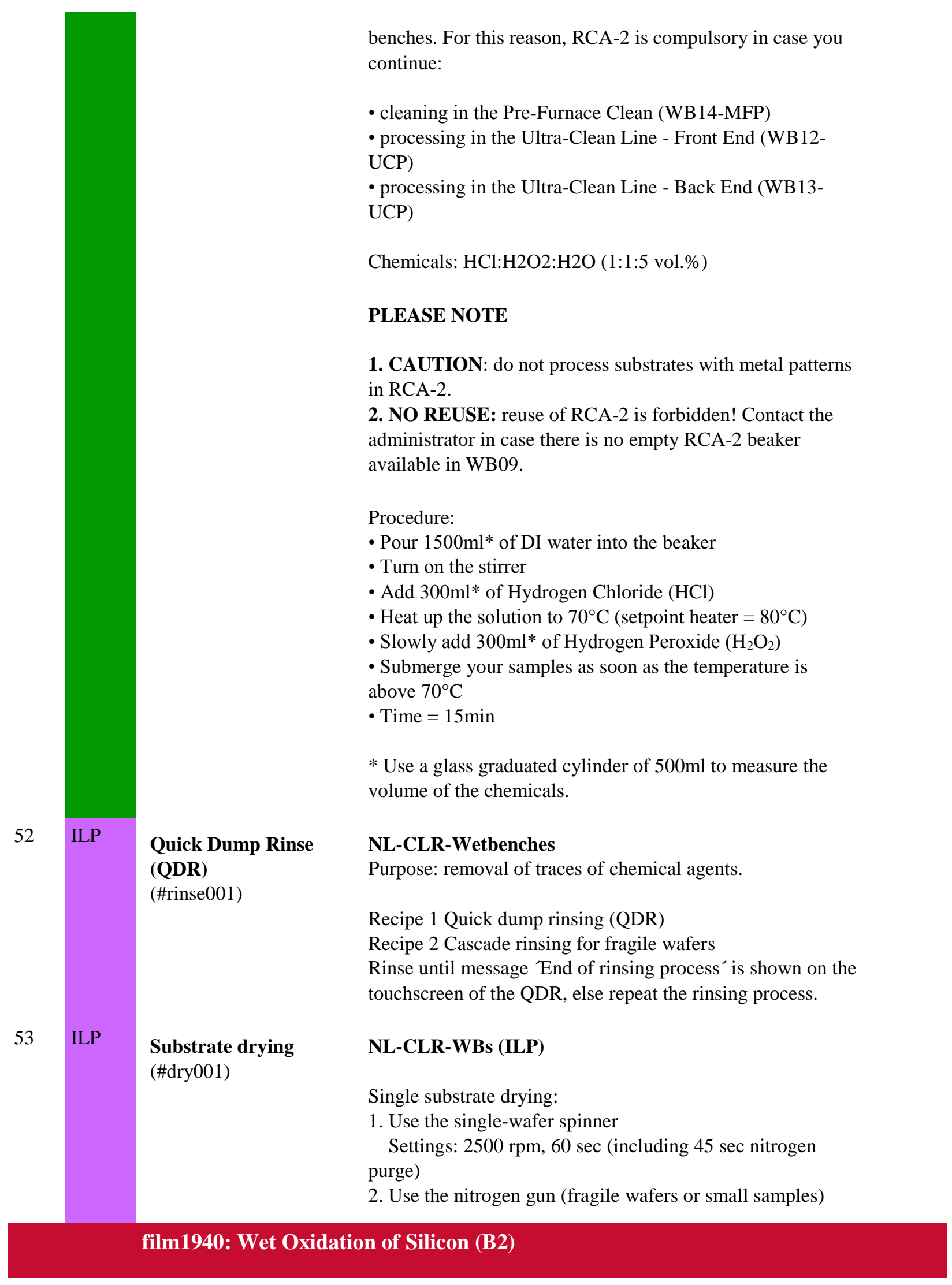




\begin{tabular}{|c|c|c|c|}
\hline 54 & MFP & $\begin{array}{l}\text { Cleaning in } 99 \% \mathrm{HNO}_{3} \\
(\# \text { clean001) }\end{array}$ & $\begin{array}{l}\text { NL-CLR-WB14 } \\
\text { Purpose: removal of organic traces. } \\
\text { - Beaker 1: } 99 \% \mathrm{HNO}_{3} \\
\text { - Time }=5 \mathrm{~min}\end{array}$ \\
\hline 55 & MFP & $\begin{array}{l}\text { Cleaning in } 99 \% \mathrm{HNO}_{3} \\
(\# \text { clean002) }\end{array}$ & $\begin{array}{l}\text { NL-CLR-WB14 } \\
\text { Purpose: removal of organic traces. } \\
\text { - Beaker } 2: 99 \% \mathrm{HNO}_{3} \\
\text { - Time }=5 \mathrm{~min}\end{array}$ \\
\hline 56 & MFP & $\begin{array}{l}\text { Quick Dump Rinse } \\
\text { (QDR) } \\
\text { (\#rinse002) }\end{array}$ & $\begin{array}{l}\text { NL-CLR-Wetbenches } \\
\text { Purpose: removal of traces of chemical agents. } \\
\text { Recipe } 1 \text { Quick dump rinsing (QDR) } \\
\text { Recipe } 2 \text { Cascade rinsing for fragile wafers } \\
\text { Rinse until message 'End of rinsing process' is shown on the } \\
\text { touchscreen of the QDR, else repeat the rinsing process. }\end{array}$ \\
\hline 57 & MFP & $\begin{array}{l}\text { Cleaning in } 69 \% \\
\text { HNO }_{3}\left(95^{\circ} \mathrm{C}\right) \\
(\# \text { clean003) }\end{array}$ & $\begin{array}{l}\text { NL-CR-WB14 } \\
\text { Purpose: removal of metallic traces. } \\
\text { - Beaker } 3 \mathrm{~A} \text { or } 3 \mathrm{~B}: 69 \% \mathrm{HNO}_{3} \\
\text { - Temperature }=95{ }^{\circ} \mathrm{C} \\
\text { - Time }=10 \mathrm{~min}\end{array}$ \\
\hline 58 & MFP & $\begin{array}{l}\text { Quick Dump Rinse } \\
\text { (QDR) } \\
\text { (\#rinse002) }\end{array}$ & $\begin{array}{l}\text { NL-CLR-Wetbenches } \\
\text { Purpose: removal of traces of chemical agents. } \\
\text { Recipe } 1 \text { Quick dump rinsing (QDR) } \\
\text { Recipe } 2 \text { Cascade rinsing for fragile wafers } \\
\text { Rinse until message 'End of rinsing process' is shown on the } \\
\text { touchscreen of the QDR, else repeat the rinsing process. }\end{array}$ \\
\hline 5 & MFP & $\begin{array}{l}\text { Substrate drying } \\
\text { (WB14) } \\
(\# \text { dry022) }\end{array}$ & $\begin{array}{l}\text { NL-CLR-WB14 } \\
\text { Optional drying step. After the QDR, you can transfer your } \\
\text { substrates directly to a Teflon carrier and strip the native } \\
\text { SiO2 in } 1 \% \text { HF (WB15). } \\
\text { Single substrate drying: } \\
\text { 1. Use the single-wafer spinner } \\
\text { Settings: } 2500 \mathrm{rpm}, 60 \mathrm{sec} \text { (including } 45 \text { sec nitrogen } \\
\text { purge) } \\
\text { 2. Use the nitrogen gun (fragile wafers or small samples) } \\
\text { Batch drying of substrates: } \\
\text { The Semitool uses the following standard procedure: }\end{array}$ \\
\hline
\end{tabular}




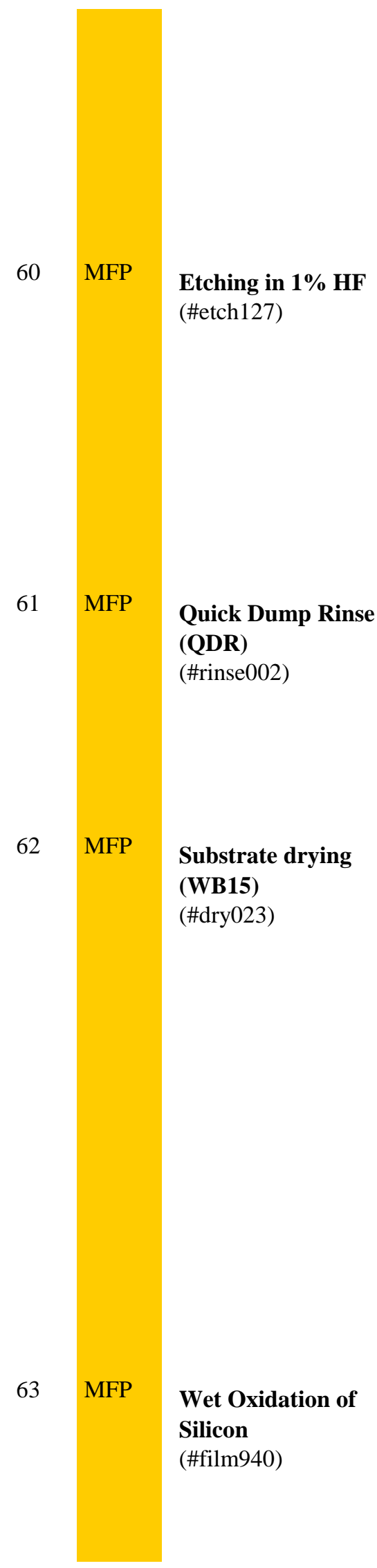

- Rinse: $30 \mathrm{sec}(600 \mathrm{rpm})$

- Q-rinse: $10.0 \mathrm{M} \Omega(600 \mathrm{rpm})$

- Purge: $10 \mathrm{sec}(600 \mathrm{rpm})$

- Drying: $280 \mathrm{sec}(1600 \mathrm{rpm})$

Note: it is obligatory to apply a single rinsing step in the QDR before using the Semitool!

NL-CLR-WB15 $\quad 50 \%$

Purpose: remove native $\mathrm{SiO} 2$ from silicon. $\mathrm{HF}$

Beaker: $1 \% \mathrm{HF}$

Temperature: room temperature

Time $=1 \mathrm{~min}$

instead

of $1 \%$

to strip

the

This step is obligatory for the MESA+ monitor wafer (if applicable, see Equipment database).

$\mathrm{SiO} 2$

layer, time: 1

$\mathrm{m}$

\section{NL-CLR-Wetbenches}

Purpose: removal of traces of chemical agents.

Recipe 1 Quick dump rinsing (QDR)

Recipe 2 Cascade rinsing for fragile wafers

Rinse until message 'End of rinsing process' is shown on the touchscreen of the QDR, else repeat the rinsing process.

\section{NL-CLR-WB15}

\section{Single substrate drying:}

1. Use the single-wafer spinner

Settings: $2500 \mathrm{rpm}, 60 \mathrm{sec}$ (including $45 \mathrm{sec}$ nitrogen purge)

2. Use the nitrogen gun (fragile wafers or small samples)

\section{Batch drying of substrates:}

The Semitool uses the following standard procedure:

- Rinse: $30 \mathrm{sec}$ (600 rpm)

- Q-rinse: $10.0 \mathrm{M} \Omega$ (600 rpm)

- Purge: $10 \mathrm{sec}(600 \mathrm{rpm})$

- Drying: $280 \mathrm{sec}(1600 \mathrm{rpm})$

Note: it is obligatory to apply a single rinsing step in the QDR before using the Semitool!

\section{NL-CLR-B2 Furnace}

Recipe: WETxxxC $(\mathrm{xxx}=$ temperature setting $)$

Oxidat

ion

step to

Settings:

- Standby temperature: $800^{\circ} \mathrm{C}$

remov

e

"nano 
- Temperature range: $800-1150^{\circ} \mathrm{C}$

- Ramp: $10^{\circ} \mathrm{C} / \mathrm{min}$

- $\mathrm{N}_{2} / \mathrm{H}_{2} \mathrm{O}$ flow: 1slm (Bubbler)

Please mention the following settings in the User Comments:

- Target thickness:

- Temperature: ...... ${ }^{\circ} \mathrm{C}$

- Time: .....min wires"

in

combi

nation

with

HF

etch.

Target

thickne

ss: 500

Tempe

rature:

1150

Time:

$26 \mathrm{~m}$
$65 \quad$ ILP

Layer thickness measurement (\#metro401)

\section{NL-CLR-Woollam M-2000UI ellipsometer}

Consult the user manual to perform a single point or a raster measurement. Use one of the available optical models to determine the layer thickness and optical constants of the coating on your substrate. Provide the following results in the digital logbook: thickness, refractive index (n) at $632.8 \mathrm{~nm}$ and the nonuniformity of the layer (\%range) of a 5point scan.

\section{etch1205: Etching in 50\% HF (WB02/09/10-private use)}

Substrate drying (\#dry001)

(QDR)

(\#rinse001)
NL-CLR-WB9/10

Use private beaker

HF 50\% standard

Temp.: room temperature

- Si3N4-H2 = $0.64 \mathrm{~nm} / \mathrm{min}$

- $\mathrm{SiRN}-\mathrm{G}^{\#}($ nanolab$)=3.1-3.5 \mathrm{~nm} / \mathrm{min}$

- $\mathrm{SiO}_{2}=1 \mu \mathrm{m} / \mathrm{min}$

\section{NL-CLR-Wetbenches}

Purpose: removal of traces of chemical agents.

Recipe 1 Quick dump rinsing (QDR)

Recipe 2 Cascade rinsing for fragile wafers

Rinse until message 'End of rinsing process' is shown on the touchscreen of the QDR, else repeat the rinsing process.

\section{NL-CLR-WBs (ILP)}

Single substrate drying:

1. Use the single-wafer spinner time:

$1 \mathrm{~m}$ 
Settings: $2500 \mathrm{rpm}, 60 \mathrm{sec}$ (including $45 \mathrm{sec}$ nitrogen purge)

2. Use the nitrogen gun (fragile wafers or small samples)

strip1400: Stripping of resists in Piranha (WB09)

69

Stripping polymers and resists

(\#strip400)

70

ILP Quick Dump Rinse (QDR)

(\#rinse001)

71

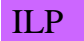

Substrate drying

(\#dry001)
NL-CLR-WB09

Purpose: stripping of polymers and resists in Piranha.

$\mathrm{H}_{2} \mathrm{SO}_{4}: \mathrm{H}_{2} \mathrm{O}_{2}$ (3:1) vol\%

Procedure:

- Put $1500 \mathrm{ml}$ Sulfuric acid $\left(\mathrm{H}_{2} \mathrm{SO}_{4}\right)$ in the beaker

- SLOWLY ADD $500 \mathrm{ml}$ Hydrogen peroxide $\left(\mathrm{H}_{2} \mathrm{O}_{2}\right)$ in order to avoid a temperature rise above $95^{\circ} \mathrm{C}$

- Start cleaning your samples at a temperature of $95^{\circ} \mathrm{C}$

- Time $=15 \mathrm{~min}$

PIRANHA IS FOR ONE-TIME USE. ALWAYS PREPARE A FRESH SOLUTION BEFORE

CLEANING YOUR SUBSTRATES!

\section{OPERATIONAL TEMPERATURE IS $95^{\circ} \mathrm{C}$.}

\section{NL-CLR-Wetbenches}

Purpose: removal of traces of chemical agents.

Recipe 1 Quick dump rinsing (QDR)

Recipe 2 Cascade rinsing for fragile wafers

Rinse until message 'End of rinsing process' is shown on the touchscreen of the QDR, else repeat the rinsing process.

\section{NL-CLR-WBs (ILP)}

Single substrate drying:

1. Use the single-wafer spinner

Settings: $2500 \mathrm{rpm}, 60 \mathrm{sec}$ (including $45 \mathrm{sec}$ nitrogen purge)

2. Use the nitrogen gun (fragile wafers or small samples) togethe

r with

glass

wafer,

time:

$15 \mathrm{~m}$

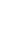

\section{.}


CHAPTER 7 


\title{
8 \\ Electrochemical study of photocatalytic reforming of biomass
}

\begin{abstract}
Hydrogen can be sustainably produced from biomass using Photocatalytic Reforming (PhCR). In this process, only sunlight is required as an external energy source to form hydrogen on the surface of a semiconductor material. PhCR is still in its infancy, so that many aspects of this process are unknown and, therefore, currently under investigation. Here, the catalyst reforming potential (bandgap) and the $\mathrm{PhCR}$ reaction mechanism of four biomass model solutions (10 wt $\%$ of ethylene glycol, glycerol, xylose or xylitol in MilliQ) were studied in a beaker set-up using cyclic voltammetry. HPLC analysis was used to analyze the liquid phase products. Ethylene glycol and xylose likely react at the catalytic surface, corresponding to the mechanism proposed in the literature. Next, a microfluidic device to study PhCR was designed and fabricated.
\end{abstract}




\subsection{Introduction}

Hydrogen is receiving increasing attention as a renewable energy vector to eventually replace fossil fuels. ${ }^{1-2}$ However, for hydrogen to be truly sustainable, the energy required to produce it, must come from renewable energy resources, such as sunlight, wind or hydrothermal energy. In this context, sunlight is investigated as a driving force for hydrogen production.

A well-known example of a photocatalytic reaction is water-splitting, where a water molecule is decomposed into hydrogen and oxygen. ${ }^{4-5}$ A semiconductor material that acts as the catalytic material is a key component in this reaction. In semiconductor materials, such as $\mathrm{TiO}_{2}{ }^{7-8}$ an electron $\left(\mathrm{e}^{-}\right)$is promoted from the valence band $(\mathrm{VB}$, the highest occupied molecular orbital or HOMO), to the conduction band ( $\mathrm{CB}$, lowest unoccupied molecular orbital or LUMO), when the irradiated sunlight is equal or higher in energy than the catalyst bandgap (Schemes 8.1A. and B.). A positive charge, or hole $\left(\mathrm{h}^{+}\right)$, is left behind in the LUMO, whereas the higher energy level becomes negatively charged by the promoted electron. This process is called charge separation. Each of the charges can subsequently take part in a redox half-reaction.
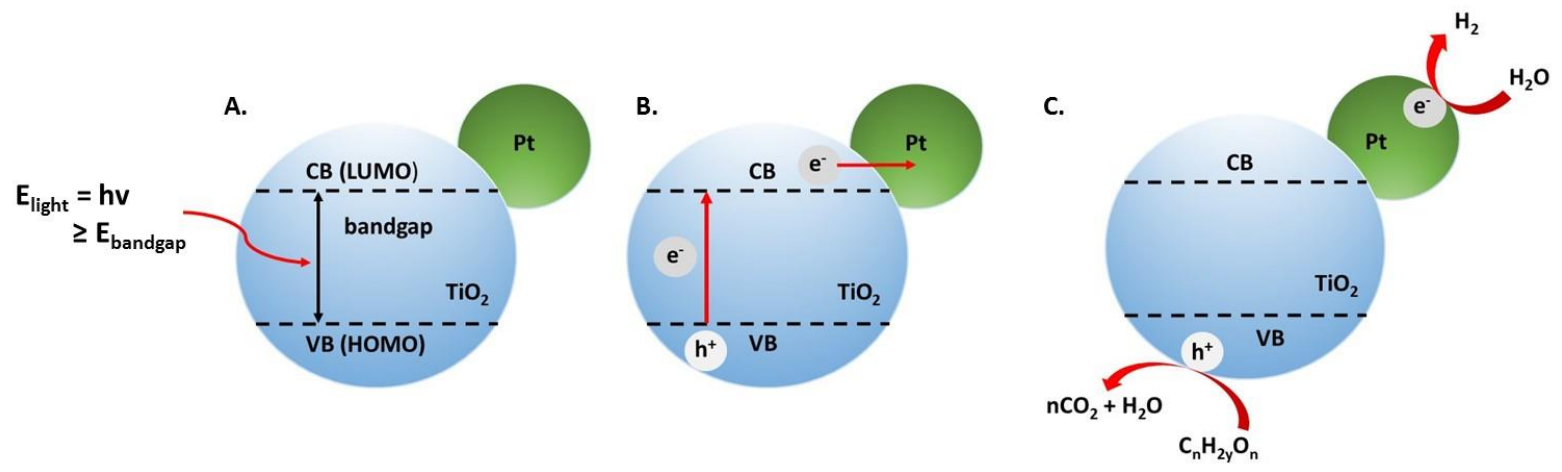

Scheme 8.1 Principle of photocatalytic reforming. In the first two steps, light with an energy equal or higher than the semiconductor bandgap promotes an electron (e) from the valence band (VB) to the conduction band (CB), leaving behind a positively charged hole $\left(\mathrm{h}^{+}\right)(\mathrm{A}$.). The electron from the $\mathrm{CB}$ diffuses to the platinum, preventing electron-hole recombination (B.). Water is reduced at the Pt side, whereas the biomass $\left(\mathrm{C}_{n} \mathrm{H}_{2 y} \mathrm{O}_{n}\right)$ is oxidized at the remaining hole at the $\mathrm{TiO}_{2}$ semiconductor (C.). Adapted from Melo et al. ${ }^{3}$

Typically, $\mathrm{Pt}$, but also $\mathrm{Cu}, \mathrm{Pd}$ and $\mathrm{Au}$, is added as a co-catalyst to a $\mathrm{TiO}_{2}$ support. ${ }^{9-10}$ The metal/semiconductor bond forms a Schottky barrier, so that the probability of the recombination of the electron and hole is minimized. ${ }^{3,11}$ 
Water splitting occurs via two half-reactions, in which the negative charge resides on the cathode, where $\mathrm{H}^{+}$is reduced to $\mathrm{H}_{2}$, while $\mathrm{OH}^{-}$is oxidized into $\mathrm{O}_{2}$ and $\mathrm{H}_{2} \mathrm{O}$ at the positively charged anode (Scheme 8.2). Overall, per mole of water, 2 moles of $\mathrm{H}_{2}$ and half a mole of $\mathrm{O}_{2}$ are produced.

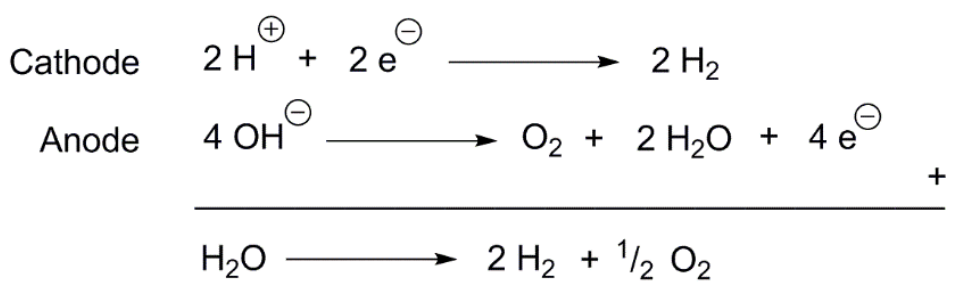

Scheme 8.2 Half-reactions for water splitting

Although hydrogen production from water splitting is highly promising, this process suffers from many limitations. The Gibbs free energy for this reaction is $+238 \mathrm{~kJ}$ per mole of $\mathrm{H}_{2} \mathrm{O},{ }^{3}$ indicating a thermodynamically unfavored process. Also, hydrogen and oxygen form an explosive mixture and therefore must be separated by an ion-selective membrane, ${ }^{12}$ so that the half-reactions take place on different sides of the membrane: the positive ions are still permeating through the membrane and between the cathode and anode, but the gaseous products form separately at the cathode and anode. ${ }^{13}$ This membrane is often not only fragile, but it could also result in diffusion limitations ${ }^{14}$ and therewith higher overpotentials for the redox reaction.

Circumventing these issues, Photocatalytic Reforming (PhCR) is based on the same principle as water splitting (Scheme 8.3). Similarly, hydrogen is formed at the anode, but biomass derivatives instead of hydroxide ions are oxidized at the cathode (Scheme 8.1.C.). ${ }^{3,14}$ The biomass can consist of otherwise discarded waste streams, ${ }^{12}$ but also of pollutants in the water. Therefore, PhCR not only produces hydrogen, but also contributes to cleaning the water. In addition, the products of this reaction, $\mathrm{CO}_{2}$ and $\mathrm{H}_{2}$, do not form an aggressive mixture. Furthermore, $\mathrm{PhCR}$ is thermodynamically favored compared to water splitting, as the Gibbs free energy is less positive. ${ }^{3,}$ 15

$$
\mathrm{C}_{n} \mathrm{H}_{2 y} \mathrm{O}_{\mathrm{n}}+\mathrm{nH}_{2} \mathrm{O} \underset{\text { catalyst }}{\stackrel{\mathrm{hv}}{\longrightarrow}} \mathrm{nCO}_{2}+(\mathrm{y}+\mathrm{n}) \mathrm{H}_{2}
$$

Scheme 8.3 Photocatalytic reforming of biomass derivatives 
So far, the required potential for PhCR (bandgap) remains unknown and the exact reaction mechanism is still a matter of debate. However, hydroxyl radicals are likely to play an important role in the reforming process. ${ }^{15-17}$ A microreactor would be ideal to study these aspects: in microreactors, compared to conventional microscale reactors, light has a path length in the order of micrometers to cover before reaching the catalyst. ${ }^{18-19}$ As a result, the light intensity is more optimally used in microscale reactors.

In this chapter, the activity of four model substrates, ethylene glycol, glycerol, xylose and xylitol towards photocatalytic reforming and the associated reaction mechanism were investigated. The required bandgap of the semiconductor catalyst as well as the reaction mechanism were studied by electrochemical methods employing cyclic voltammetry in a beaker set-up. In parallel, several microfluidic devices were designed and fabricated to analyze the products of the half-reactions separately and to eventually elucidate the PhCR reaction mechanism.

\subsection{Materials and Methods}

\subsubsection{Biomass model solutions}

Four biomass model substrate solutions were prepared, consisting of $1 \mathrm{M}$ of either ethylene glycol, glycerol, D-xylose or xylitol (all purchased from Sigma-Aldrich) in MilliQ water. The substrates were used as received. MilliQ water without any substrate acted as a reference. To all solutions $0.5 \mathrm{M} \mathrm{H}_{2} \mathrm{SO}_{4}$ was added until a $\mathrm{pH}$ of 0.5 was reached to ensure electrical conductivity of the solution.

\subsubsection{Cyclic voltammetry and electrochemical conversion in a beaker set-up}

Two Pt-wires (0.25 $\mathrm{mm}$ in diameter) acting as the working and counter electrodes were placed 2 $\mathrm{cm}$ from each other and submerged by $3.4 \mathrm{~cm}$ in a $30 \mathrm{~mL}$ beaker. A reference electrode, $\mathrm{Ag} / \mathrm{AgCl}$ in a $3 \mathrm{M} \mathrm{KCl}$ solution, was added to ensure reliable and reproducible results. Voltammograms were recorded using a PalmSens potentiostat in a 3-electrode mode at a scan rate of $50 \mathrm{mV} . \mathrm{s}^{-1}$ and at room temperature. To evaluate conversion and liquid phase product formation, a potential of 3 $\mathrm{V}$ was applied using an external power supply (TENMA 72-2935) to the solution at room temperature. Samples were taken after 30 minutes and subsequently every hour, for up to 6 hours. 
The samples were analyzed by HPLC (Aminex HPX-87H column, refractive index detector) at Utrecht University.

To test the microfluidic device and its gas/liquid separation efficiency, biomass model solutions were infused at a flow rate of $100 \mu \mathrm{L} \cdot \mathrm{min}^{-1}$ and a potential of $3 \mathrm{~V}$ was applied between the two electrodes to simulate the potential of the $\mathrm{Pt} / \mathrm{TiO}_{2}$ catalyst. The $\mathrm{TiO}_{2}$ bandgap is $3.2 \mathrm{eV}$, ${ }^{20}$ which can be reduced to $2.9 \mathrm{eV}$ (3\% wt Pt loading) ${ }^{21}$ or even $2.7 \mathrm{eV}$ (1.7 wt $\%$ Pt loading). ${ }^{22}$ Optical microscopy was used to monitor the gas formation and gas/liquid separation in the microreactor.

\subsection{Results and discussion}

\subsubsection{Cyclic voltammetry}

Figure 8.1 presents the cyclic voltammograms obtained for the four biomass model solutions, recorded at a scan rate of $50 \mathrm{mV} \cdot \mathrm{s}^{-1}$. The potential was applied in a range of 0 to $1.23 \mathrm{~V}$, which corresponds to the reduction and oxidation potential of water. ${ }^{4,}{ }^{12}$ Applying a higher potential would lead to a substantial amount of gas formation on the electrodes due to water splitting, which would compromise the accuracy of the measurements. Positive currents indicate oxidation, whereas negative currents refer to reduction. The potential at the $x$-axis has been recalculated as a function of the reverse hydrogen electrode (RHE) for comparison with literature data according to:

$$
E(R H E)=E_{A g / A g C l}+0.059 p H+E_{A g / A g C l}^{\theta}
$$

with $E_{A g / A g C l}^{\theta}$ being the standard potential of the $\mathrm{Ag} / \mathrm{AgCl}$ electrode of 0.199 and a $\mathrm{pH}$ of 0.5 . On the $y$-axis, the measured activity of the substrates is expressed as the current per $\mathrm{cm}^{2} \mathrm{Pt}$-electrode surface area.

All four graphs are asymmetric at the $x$-axis, indicating that the substrate reacts at the electrode surface instead of in the solution. For all four substrates a large negative peak was observed at $0.026 \mathrm{~V} v s$. RHE, which corresponds to the half reaction producing hydrogen in an acidic environment. ${ }^{23}$ Apart from this peak, glycerol and xylose did not show any activity in the scanned potential range. 
A.

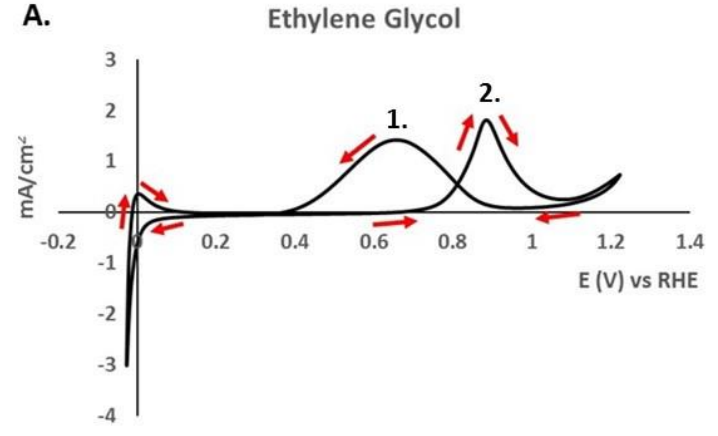

c.

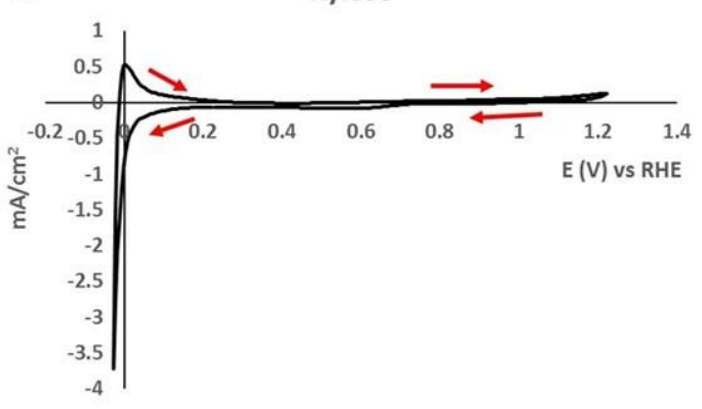

B.

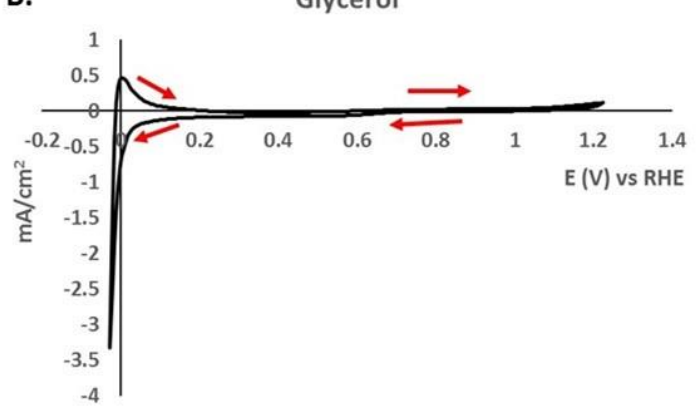

D.

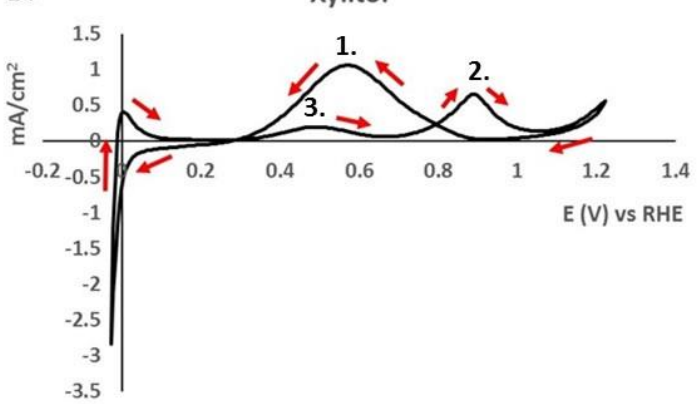

Figure 8.1 Cyclic voltammograms for $1 \mathrm{M}$ solutions of ethylene glycol (A.), glycerol (B.), xylose (C.) and xylitol (D.) The large negative peak in all four graphs at $0.026 \mathrm{~V}$ indicates the half reaction for hydrogen production in an acidic environment.

However, additional peaks were observed in the voltammograms of ethylene glycol (Figure 8.1 A.) and xylitol (Figure 8.1 D.), which are indicated with numbers (See also Table 8.1). The voltammogram of ethylene glycol was in good agreement with literature data, ${ }^{24}$ where a similarly shaped graph was reported. The voltammograms of xylitol and ethylene glycol are comparable, both showing two oxidation peaks (ethylene glycol peaks 1 and 2 and xylitol peaks 1 and 2), which could suggest a similar reforming mechanism. Apart from the negative hydrogen production peak at $0.026 \mathrm{~V}$, almost all remaining peaks can be attributed to $\mathrm{CO}$ oxidation. ${ }^{24-25}$ Peak 3 of xylitol could indicate $\mathrm{CO}$ surface poisoning. ${ }^{26}$

\subsubsection{Mechanism of PhCR}

Based on these $\mathrm{CV}$ measurements, some information on the (electrochemical) reaction mechanisms can be derived. A mechanism involving adsorption of the biomass substrate on the catalytic surface now seems to be the most likely, as illustrated in Scheme 8.4. ${ }^{6,15}$ 
Table 8.1 Peaks demonstrating substrate activity during cyclic voltammetry measurements. The different peaks and their possible attribution are indicated for the used model substrates.

\begin{tabular}{lccc}
\hline Substrate & Peak number & Potential (V) vs RHE & Indicating \\
\hline All substrates & & 0.026 & Hydrogen production \\
Ethylene glycol (EG) & 1 & 0.653 & CO oxidation \\
& 2 & 0.889 & CO oxidation \\
Xylitol (XOL) & 1 & 0.569 & CO oxidation \\
& 2 & 0.889 & CO oxidation \\
& 3 & 0.494 & CO surface poisoning \\
\hline
\end{tabular}

An electron of a primary hydroxyl group of the biomass substrate recombines with a hole at the anode side of the catalyst. Simultaneously, a proton of the same hydroxyl group is abstracted by the negative charge of the catalyst, releasing an alkoxide radical derivative of the substrate. This radical subsequently reacts with another substrate molecule, yielding an alcohol and an alcohol radical. The alcohol radical can then coordinate with the catalyst in a similar fashion as in the first reaction step, yielding an aldehyde and a proton. In parallel, water reacts with a hole on the catalytic surface, forming a proton and a hydroxyl radical. The aldehyde reacts with this hydroxyl radical to form an acid intermediate, which is photocatalytically reformed into $\mathrm{CO}_{2}$ and $\mathrm{H}_{2}$.

In this mechanism, the half-reaction taking place at the cathode is the reduction of $\mathrm{H}^{+}$, which has a standard potential of $0.0 \mathrm{~V} v s$. RHE. The half-reaction at the anode produces a hydroxyl radical, which has a standard potential of $+2.27 \mathrm{~V}$. ${ }^{3}$ Therefore, when a potential of $2.27 \mathrm{~V}$ is applied during cyclic voltammetry measurements, the biomass substrate can be reformed until the formation of the aldehyde intermediate. The standard potential for substrate oxidation when absorbed on the catalytic surface, is likely in the order of -1.00 to $-0.50 \mathrm{~V}$. Liu et al. ${ }^{27}$ reported $-0.65 \mathrm{~V}$ and -0.72 $\mathrm{V}$ for the oxidation of 1-propanol and 2-propanol respectively on a similar Pt-electrode as used in this study. The theoretical standard cell potential for this reaction step ranges from +0.50 to +1.00 $\mathrm{V}$, which is in the range of the $\mathrm{CV}$ measurements performed in this chapter. Based on these data, and the fact that xylose and glycerol did not show any activity during the $\mathrm{CV}$ measurements, these substrates are either inactive for photocatalytic reforming, or react via a different mechanism. 


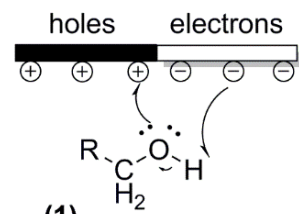

(1)

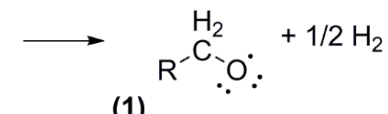

(1)
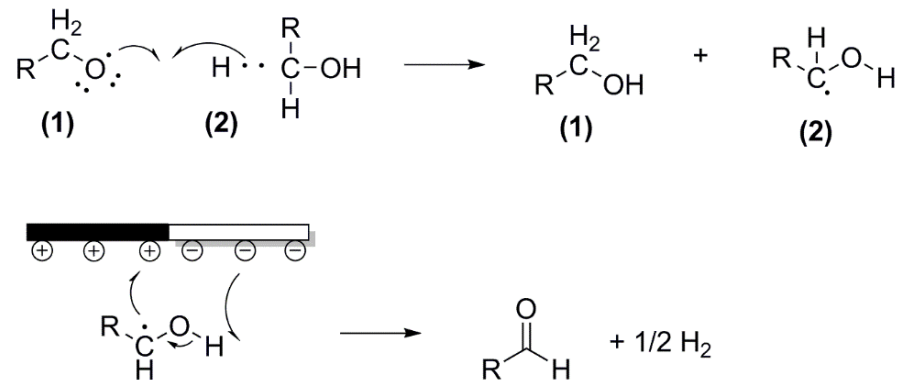

(2)

(3)

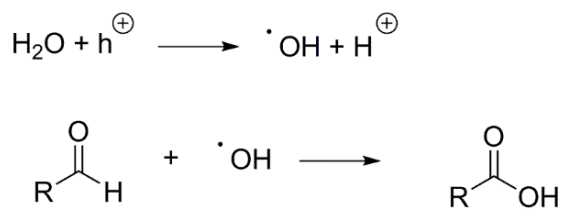

(3)

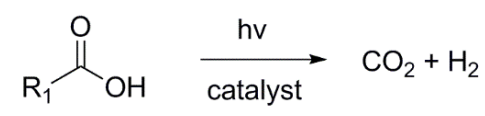

Scheme 8.4 Proposed mechanism for photocatalytic reforming of alcohols and sugars. The biomass substrate coordinates to the hole at the catalytic surface, interacting directly with the catalyst. ${ }^{6}$ Next, the resulting biomass radical reacts with a second biomass molecule. The substrate biomass undergoes several radical reactions until an aldehyde is formed. This aldehyde subsequently reacts with another hydroxyl radical, forming an acid. This acid is photocatalytically transformed into hydrogen and $\mathrm{CO}_{2}$.

However, ethylene glycol and xylitol activity was observed during CV experiments, indicating that these substrates could be reformed following the mechanism described above.

\subsubsection{Biomass conversion}

To confirm the reactivity of ethylene glycol (EG), the conversion of the substrate was evaluated by applying $3 \mathrm{~V}$ to the solution in a beaker set-up, followed by HPLC analysis of the liquid phase (Figure 8.2). At least two products were formed after 6 hours of reaction, indicated with A. and B. The area-under-the-curve (A.U.C.) directly relates to the product concentration. A rough estimate of $12 \%$ EG conversion was calculated, taking into account the total charge that passed through the 


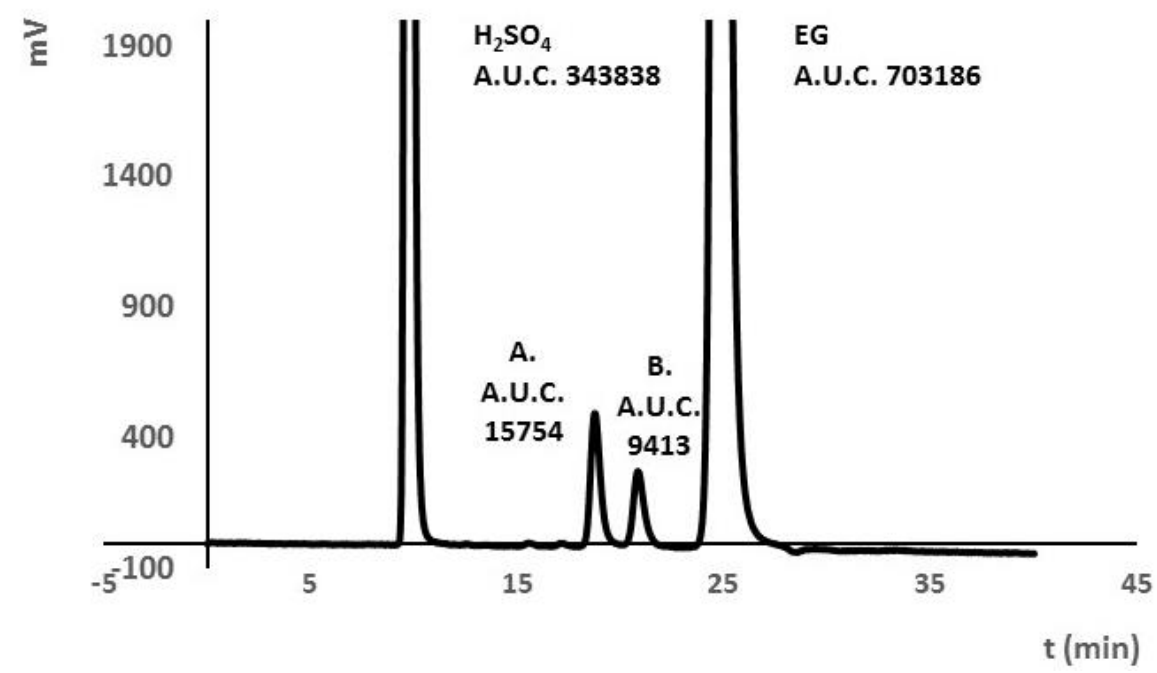

Figure 8.2 HPLC analysis of the liquid phase of a $1 \mathrm{M} \mathrm{EG}$ substrate solution after applying $3 \mathrm{~V}$ for 6 hours. The area under the curve (A.U.C.) is indicated. Four peaks were found compared to the HPLC chromatogram recorded at $\mathrm{t}=0 \mathrm{~min}$, which corresponded to $\mathrm{H}_{2} \mathrm{SO}_{4}$ which was added to ensure electrical conductivity, two product peaks (A. and B.) and ethylene glycol (EG).

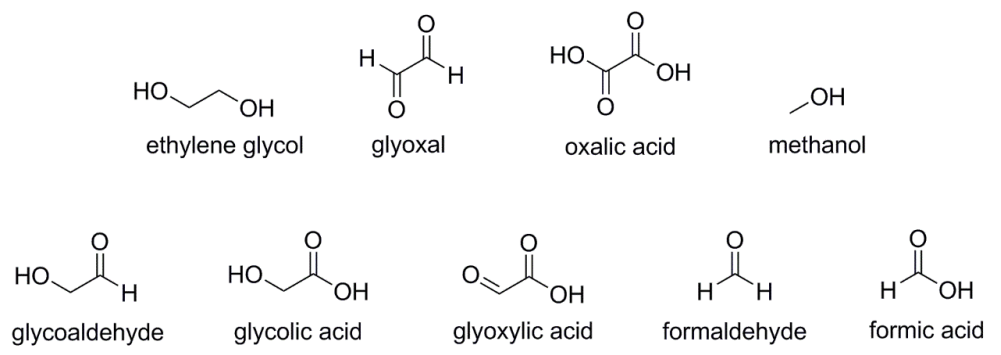

Figure 8.3 Possible liquid phase intermediates formed during photocatalytic reforming of ethylene glycol.

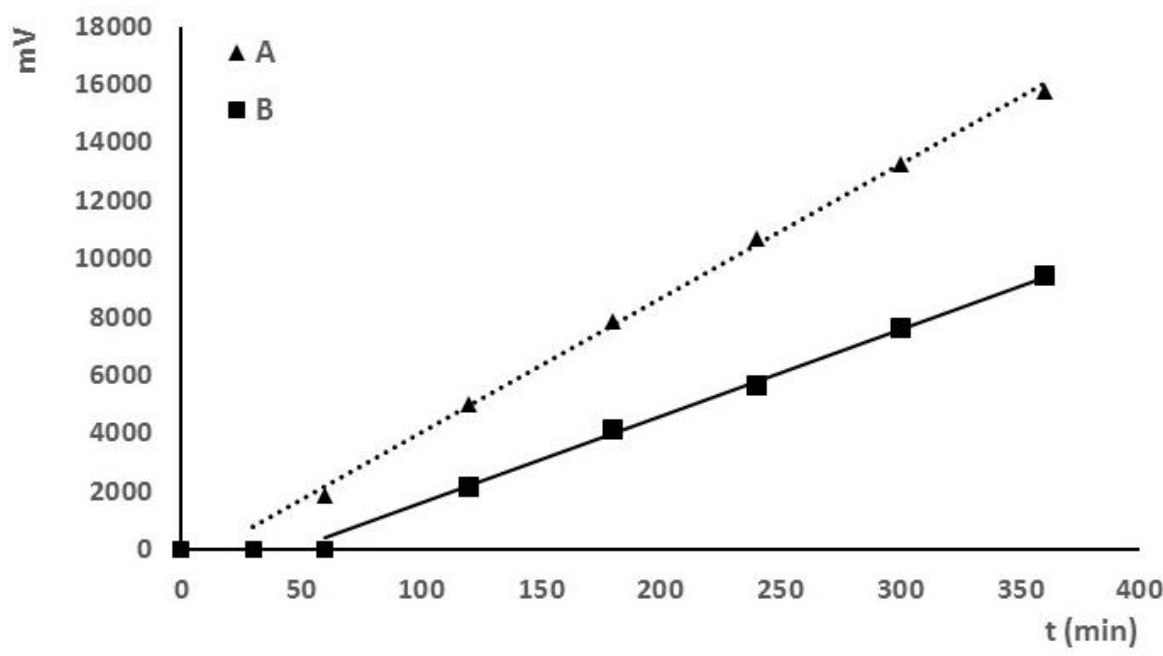

Figure 8.4 Concentration of products A. and B. as a function of time. 
solution and assuming 100\% Faradaic efficiency, which indicates that PhCR presents slow kinetics.

Based on the proposed mechanism, several intermediates are possible (Figure 8.3), of which most of them have also been identified experimentally in previous studies. ${ }^{25}$ The concentrations of A. and B. were monitored over 6 hours, which increased linearly over time (Figure 8.4). As the estimated ethylene glycol conversion was low, compounds A and B are probably intermediates formed early on in the reforming mechanism. As A. was detected after a shorter reaction time, it is likely to be glycoaldehyde, which is closest structurally to ethylene glycol. The aldehyde group can then react to form the corresponding acidic derivative, or the other hydroxyl group can react, resulting in a di-aldehyde. Based on this assumption, B. is either glycolic acid or glyoxal (Figure $8.3)$.

\subsubsection{Microfluidic device}

In addition to the beaker set-up, a microfluidic device was designed (Figure 8.5) to study the reaction mechanism and determine the required catalyst bandgap. In this device, the products of the half-reactions could be collected and analyzed separately, since the gas phase and liquid phase products could be separated in the device.

The silicon/glass microfluidic device consisted of two connected fluidic channels (in blue, 100$\mu \mathrm{m}$ deep and 500- $\mu \mathrm{m}$ wide), separated by hydrophilic microchannels of $2-\mu \mathrm{m}$ in width, $2-\mu \mathrm{m}$ in depth and $40 \mu \mathrm{m}$ in length (zoom-in, Figures 8.5 A. and B.). These microchannels allowed for free ion transport, whereas any gaseous products would not permeate (see Chapter 7 for the concept), so that the gas phase products could be analyzed separately. A larger, shared fluidic chamber was located near the inlet, to ensure equal pressure between the separated channels. The inlet and outlets are indicated in green. The reaction occurs at the Pt-contacts (gray), onto which a voltage can be applied. Larger electrical Pt-pads of $0.5 \mathrm{~cm}$ by $2 \mathrm{~cm}$ in size were fabricated on the overhanging glass layer at the long sides of the device.

Separation of the gaseous and liquid products occurred through either a PDMS membrane (Figure 8.5 A) which was placed over outlets 4 and 5, a micropillar membrane (zoom-in Figure 8.5 B.) or 

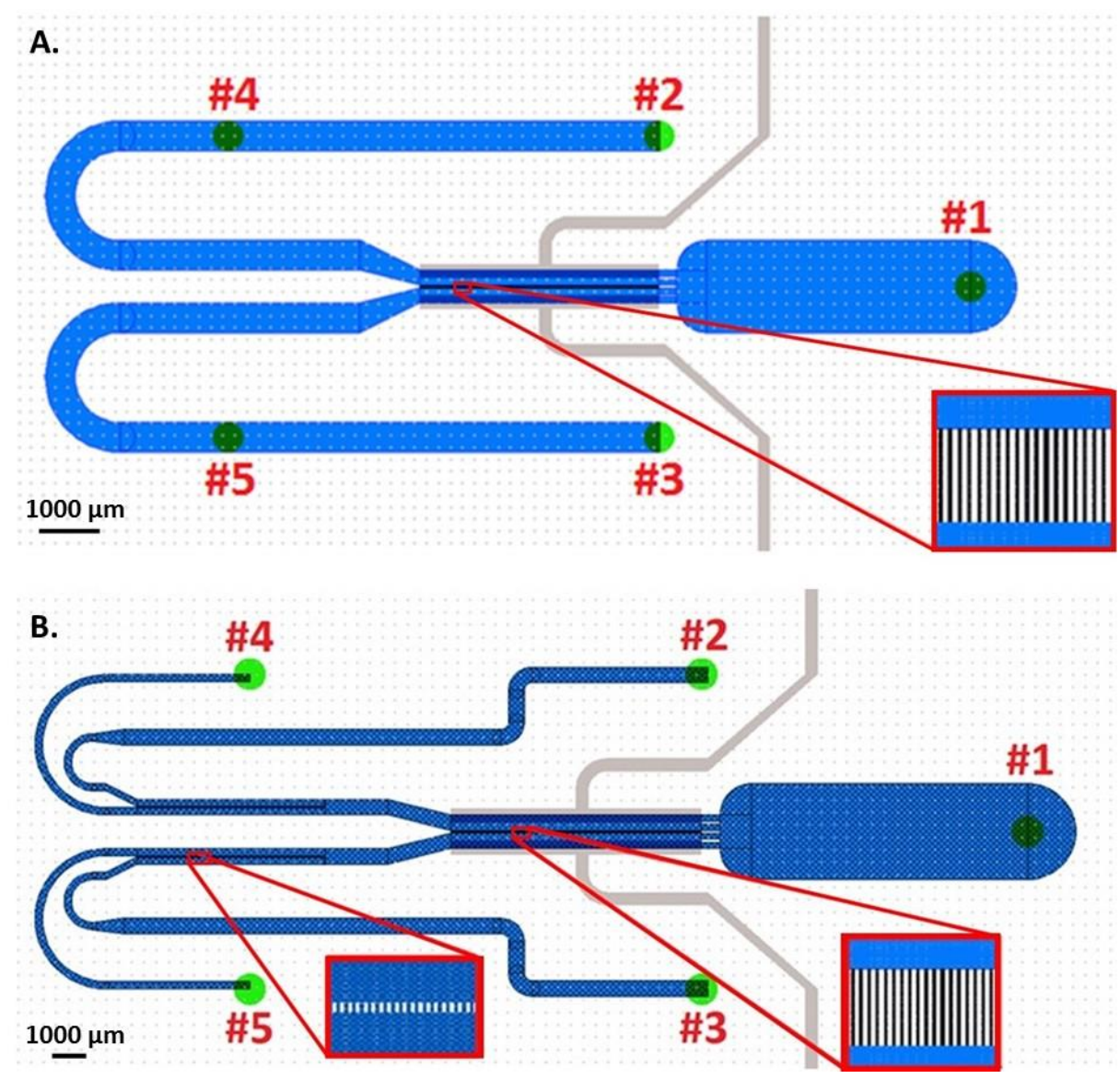

Figure 8.5 Clewin design of the microreactor for voltammetry measurements and mechanistic studies, with gas/liquid separation by means of a PDMS membrane (A.). or a micropillar array (B.) The main liquid channels, the platinum electrodes, and the gas/liquid separation channel are depicted in blue, in gray, and in black respectively. The inlet and outlets are indicated in green and numbered 1-5 with \#1 being the inlet and \#2-\#5 being outlets.

a combination of the two. The micropillars had a foot print of $5 \mu \mathrm{m}$ by $10 \mu \mathrm{m}$ and the same height as the microchannel. Liquid could permeate through the hydrophilic pillars, whereas the gas phase could not. The gaseous products could be collected at outlets 4 and 5 , and the liquid phase products at outlets 2 and 3 .

\subsubsection{Device fabrication}

\section{Silicon wafer}

A 250-nm thick layer of $\mathrm{SiO}_{2}$ was grown on a silicon $\langle 100\rangle$ wafer by wet oxidation. Next, the main liquid and separation microchannels were dry-etched into the $\mathrm{SiO}_{2}$ layer after performing 
photolithography. An embedded mask was created on top of the $\mathrm{SiO}_{2}$ by a photolithography step with a mask containing only the main channels, followed by dry-etching of the main channel 100$\mu \mathrm{m}$ deep into the silicon. The $\mathrm{SiO}_{2}$ layer was then removed and reapplied by wet oxidation to form a 250-nm thick $\mathrm{SiO}_{2}$ layer in the channels. On the back side of the silicon wafer, the in- and outlets were fabricated after photolithography, by dry-etching of the $\mathrm{SiO}_{2}$ layer and the silicon. Again, the $\mathrm{SiO}_{2}$ layer was stripped and reapplied to cover the silicon with $250-\mathrm{nm} \mathrm{SiO} \mathrm{S}_{2}$, rendering the device electrically insulated.

\section{Glass wafer}

The electrodes were manufactured on the glass layer of the microfluidic device. To ensure a flat surface, allowing the anodic bonding with the silicon layer, the electrodes were concealed into the glass layer. First, 200-nm deep structures were etched into the glass by buffered HF. Next, 5-nm tantalum was sputtered as an adhesion layer, followed by a 200-nm thick platinum layer that acted as the electrodes and the $4 \mathrm{~mm}$ by $10 \mathrm{~mm}$ Pt-contacts. After sonication, the glass and silicon layer were anodically bonded and diced into the individual devices.

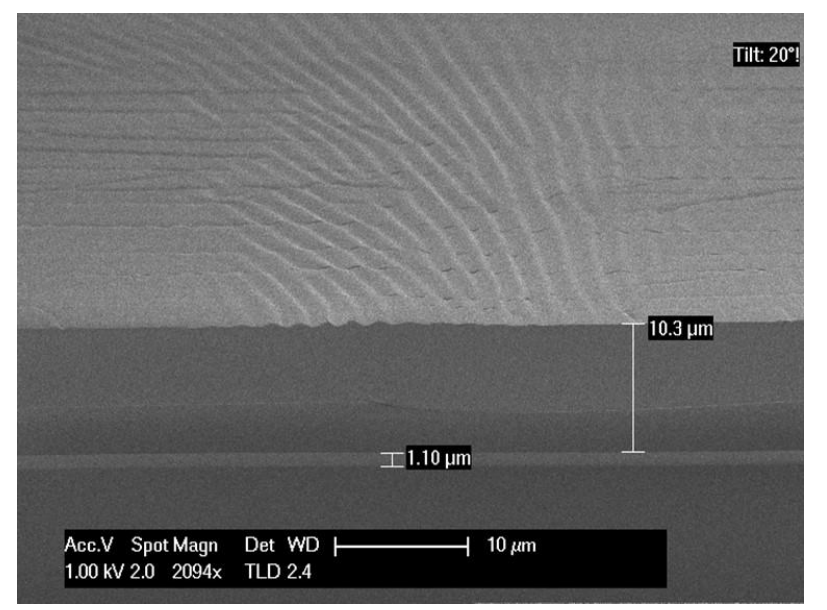

Figure 8.6 SEM image of the PDMS membrane (crosssection). Here the membrane has a uniform thickness of $10.3 \mu \mathrm{m}$ and the sacrificial mask layer has a uniform thickness of $1.1 \mu \mathrm{m}$.
Post-processing and PDMS membrane fabrication

On the silicon wafer, areas which were over the electrical contact pads were etched at the same time as the main channels. This etching created free floating sections of silicon of $5 \mathrm{~mm}$ by $20 \mathrm{~mm}$ on the two long sides of the chip after dicing. These sections were carefully removed by hand just before the experiment, which resulted in an overhanging glass section on the chip with the electrical contact pads. 
To separate the gaseous from the liquid phase products, a polydimethylsiloxane (PDMS) membrane was fabricated. PDMS precursor and curing agent were mixed at a 10:1 weight ratio and subsequently spin-coated on a silicon wafer coated with photoresist $(4000 \mathrm{rpm}, 30 \mathrm{~s})$ as a sacrificial layer. The PDMS was cross-linked in an oven at $353 \mathrm{~K}$ for 5 hours. By submerging the wafer in acetone the photoresist was dissolved, releasing the PDMS membrane. Tweezers were used to place the membrane over the designated outlets.

\subsubsection{Microfluidic set-up}

A dedicated chip holder was fabricated for the fluidic and electronic connections. PEEK was chosen as a material, as this material should not degrade during intense UV-radiation. The bottom plate contained an optical window to monitor the formation of gaseous products inside the fluidic channel. The bottom plate was connected to a top plate containing the fluidic and electronic connections, tightened by 6 screws. PEEK tubing (1/32") was screwed into the top plate with ferrules, acting as the fluidic connection. O-ring sealing should prevent any leakages. Pushpins touching the

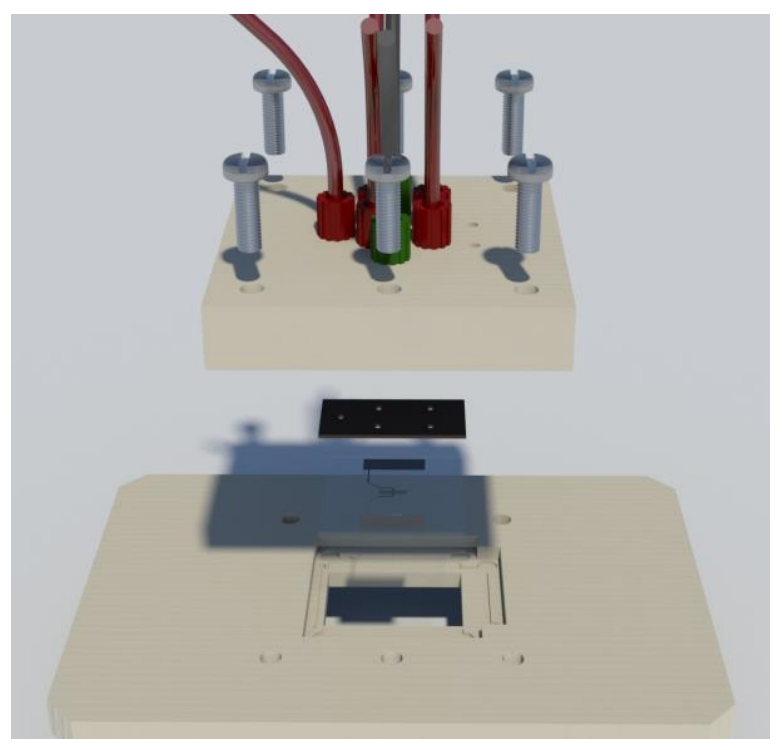

Figure 8.6 Exploded view of both the chip holder and the microfluidic device.

Pt-contacts on the microfluidic device were connected to either a potentiostat (PalmSens) during the cyclic voltammetry or to a benchtop power supply (TENMA 72-2935).

\subsubsection{Testing of the microfluidic device}

Application of $3 \mathrm{~V}$ at the Pt-electrodes of the microfluidic device resulted in gas formation in the microchannel (Figure 8.7). The fluid flow of $100 \mu \mathrm{L} \cdot \mathrm{min}^{-1}$, however, was insufficient to remove the gas bubbles from the Pt-electrodes, resulting in fluid obstruction, pressure built-up and 


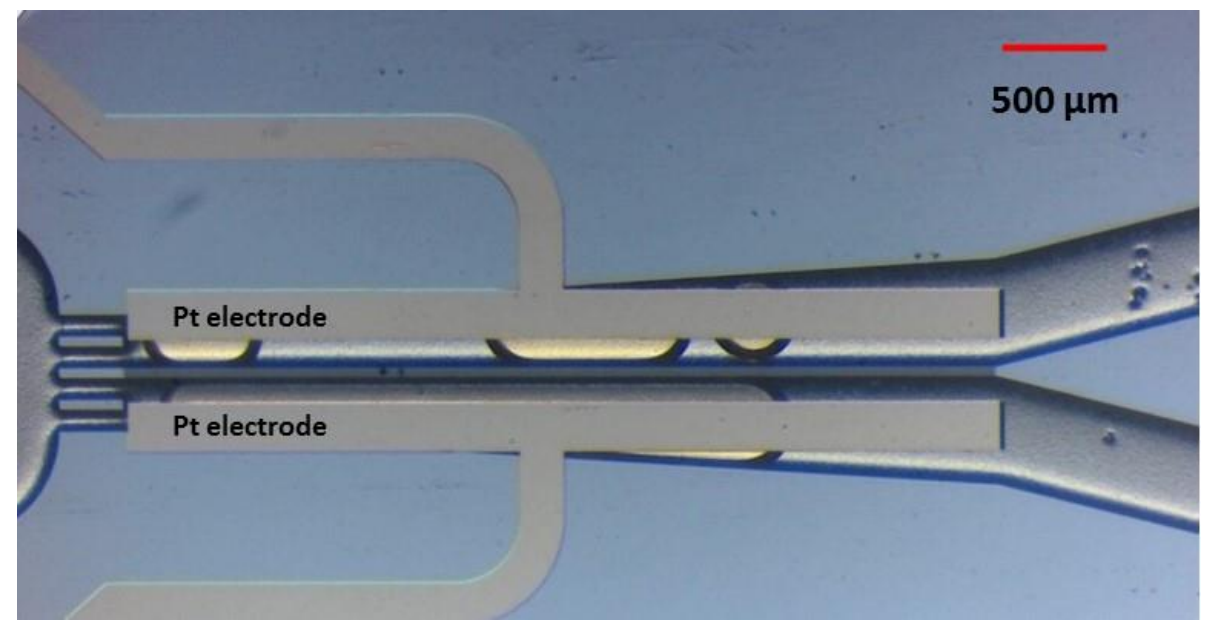

Figure 8.7 Microfluidic device with gas generation at the Pt electrodes. The bubbles are obstructing the microchannel, resulting in pressure built-up and irregular fluid flow downstream.

irregular flow velocity in the microchannel. Furthermore, the bubbles blocked the electrode for further reaction.

Compared to the gas/liquid microseparator described in Chapter 7, here, the phase separation takes place in the same device where the reaction is performed. The first separation method used an array of micropillars. During experiments, gas did not permeate the micropillar membrane, but, unfortunately, the gas stream still contained liquid. A longer separation section could solve this issue.

The second gas/liquid separation employed a PDMS membrane over the outlet of the device. No gas could be collected at the outlets: liquid filled the gap in the silicon forming the outlet, therefore blocking the path to the outlet for the passing gas phase (Figure 8.8). For the same reason, combining the micropillar array with the PDMS membrane was unsuccessful. In an improved design the membrane should be placed on the other side of the silicon that than on the one which it was now, to prevent the liquid from

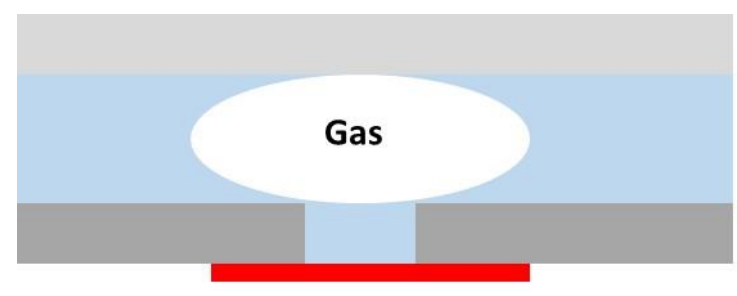

\section{Silicon \\ PDMS membrane \\ Liquid \\ Glass}

Figure 8.8 Cross-section of microchannel with an outlet covered by a PDMS membrane. Liquid filled the outlet, preventing the gas extraction by the membrane. blocking the outlet. 


\subsection{Conclusion}

In this chapter, photocatalytic reforming of biomass for renewable hydrogen production was investigated electrochemically. Both cyclic voltammetry and the electrochemical reaction were performed using $1 \mathrm{M}$ solutions of ethylene glycol, glycerol, xylose and xylitol in a beaker set-up. Only ethylene glycol and xylitol showed activity in the voltage range studied during CV $(0-1.23$ $\mathrm{V} v s$. RHE). Based on literature data and HPLC analysis of the liquid phase products, ethylene glycol was converted by $12 \%$ after 6 hours, indicating slow kinetics. Two intermediates were detected, possibly glycoaldehyde and glycolic acid or the di-aldehyde, which supported the mechanism for ethylene glycol proposed in the literature.

A first step was made to design and fabricate a microfluidic device to study $\mathrm{PhCR}$, in which the products of the half-reaction could be analyzed separately. A micropillar array and a PDMS membrane were investigated to separate the gas and liquid streams, of which the micropillar array proved to be more suitable. In an improved design, a longer separation section is suggested to fully separate the two phases.

\section{Acknowledgements}

I would like to thank Vincent de Boer for his creativity and hard work that led to the results presented in this chapter. Furthermore, I thank Dr. Fang Liu, Utrecht University, for the HPLC analysis, and Stefan Schlautmann and Hai Le The for their help during the microfabrication.

\section{References}

1. Balat, H.; Kirtay, E., Hydrogen from biomass - Present scenario and future prospects. Int. J. Hydrogen Energy 2010, 35 (14), 7416-7426.

2. Hanley, E. S.; Deane, J. P.; Gallachoir, B. P. O., The role of hydrogen in low carbon energy futures-A review of existing perspectives. Renewable \& Sustainable Energy Reviews 2018, 82, 3027-3045.

3. Melo, M. D.; Silva, L. A., Photocatalytic Production of Hydrogen: an Innovative Use for Biomass Derivatives. Journal of the Brazilian Chemical Society 2011, 22 (8), 1399-1406. 
4. Qi, J.; Zhang, W.; Cao, R., Solar-to-Hydrogen Energy Conversion Based on Water Splitting. Advanced Energy Materials 2018, 8 (5).

5. Christoforidis, K. C.; Fornasiero, P., Photocatalytic Hydrogen Production: A Rift into the Future Energy Supply. Chemcatchem 2017, 9 (9), 1523-1544.

6. $\quad$ Fu, X.; Long, J.; Wang, X.; Leung, D. Y. C.; Ding, Z.; Wu, L.; Zhang, Z.; Li, Z.; Fu, X., Photocatalytic reforming of biomass: A systematic study of hydrogen evolution from glucose solution. Int. J. Hydrogen Energy 2008, 33 (22), 6484-6491.

7. Ge, M.; Cai, J.; Iocozzia, J.; Cao, C.; Huang, J.; Zhang, X.; Shen, J.; Wang, S.; Zhang, S.; Zhang, K.-Q.; Lai, Y.; Lin, Z., A review of TiO2 nanostructured catalysts for sustainable $\mathrm{H} 2$ generation. Int. J. Hydrogen Energy 2017, 42 (12), 8418-8449.

8. Ahmad, H.; Kamarudin, S. K.; Minggu, L. J.; Kassim, M., Hydrogen from photo-catalytic water splitting process: A review. Renewable and Sustainable Energy Reviews 2015, 43, 599-610. 9. Kumaravel, V.; Mathew, S.; Bartlett, J.; Pillai, S. C., Photocatalytic hydrogen production using metal doped TiO2: A review of recent advances. Applied Catalysis B: Environmental 2019, 244, 1021-1064.

10. Melvin, A. A.; Illath, K.; Das, T.; Raja, T.; Bhattacharyya, S.; Gopinath, C. S., M-Au/TiO2 $(\mathrm{M}=\mathrm{Ag}, \mathrm{Pd}$, and $\mathrm{Pt})$ nanophotocatalyst for overall solar water splitting: role of interfaces. Nanoscale 2015, 7 (32), 13477-13488.

11. Navarro, R. M.; Sánchez-Sánchez, M. C.; Alvarez-Galvan, M. C.; Valle, F. d.; Fierro, J. L. G., Hydrogen production from renewable sources: biomass and photocatalytic opportunities. Energy \& Environmental Science 2009, 2 (1), 35-54.

12. Kuehnel, M. F.; Reisner, E., Solar Hydrogen Generation from Lignocellulose. Angew. Chem. Int. Ed. 2018, 57 (13), 3290-3296.

13. Dahl, S.; Chorkendorff, I., SOLAR-FUEL GENERATION Towards practical implementation. Nature Materials 2012, 11 (2), 100-101.

14. Chai, Z.; Zeng, T.-T.; Li, Q.; Lu, L.-Q.; Xiao, W.-J.; Xu, D., Efficient Visible Light-Driven Splitting of Alcohols into Hydrogen and Corresponding Carbonyl Compounds over a Ni-Modified CdS Photocatalyst. J. Am. Chem. Soc. 2016, 138 (32), 10128-10131.

15. Li, D.; Li, X. Y.; Gong, J. L., Catalytic Reforming of Oxygenates: State of the Art and Future Prospects. Chem. Rev. 2016, 116 (19), 11529-11653. 
16. Chang, C.-J.; Lin, Y.-G.; Weng, H.-T.; Wei, Y.-H., Photocatalytic hydrogen production from glycerol solution at room temperature by $\mathrm{ZnO}-\mathrm{ZnS} /$ graphene photocatalysts. Appl. Surf. Sci. 2018, 451, 198-206.

17. Nomikos, G. N.; Panagiotopoulou, P.; Kondarides, D. I.; Verykios, X. E., Kinetic and mechanistic study of the photocatalytic reforming of methanol over $\mathrm{Pt} / \mathrm{TiO} 2$ catalyst. Applied Catalysis B: Environmental 2014, 146, 249-257.

18. Knowles, J. P.; Elliott, L. D.; Booker-Milburn, K. I., Flow photochemistry: Old light through new windows. Beilstein Journal of Organic Chemistry 2012, 8, 2025-2052.

19. Yusuf, A.; Garlisi, C.; Palmisano, G., Overview on microfluidic reactors in photocatalysis: Applications of graphene derivatives. Catal. Today 2018, 315, 79-92.

20. Li, Z.; Luo, W.; Zhang, M.; Feng, J.; Zou, Z., Photoelectrochemical cells for solar hydrogen production: current state of promising photoelectrodes, methods to improve their properties, and outlook. Energy \& Environmental Science 2013, 6 (2), 347-370.

21. Garcia-Zaleta, D. S.; Torres-Huerta, A. M.; Dominguez-Crespo, M. A.; Garcia-Murillo, A.; Silva-Rodrigo, R.; Gonzalez, R. L., Influence of Phases Content on Pt/TiO2, Pd/TiO2 Catalysts for Degradation of 4-Chlorophenol at Room Temperature. Journal of Nanomaterials 2016.

22. Guayaquil-Sosa, J. F.; Calzada, A.; Serrano, B.; Escobedo, S.; de Lasa, H., Hydrogen Production via Water Dissociation Using Pt-TiO2 Photocatalysts: An Oxidation-Reduction Network. Catalysts 2017, 7 (11).

23. Wang, Q.; Cha, C.-S.; Lu, J.; Zhuang, L., The electrochemistry of "solid/water" interfaces involved in PEM-H2O reactors Part I. The "Pt/water" interfaces. PCCP 2009, 11 (4), 679-687.

24. Bayer, D.; Berenger, S.; Joos, M.; Cremers, C.; Tubke, J., Electrochemical oxidation of C2 alcohols at platinum electrodes in acidic and alkaline environment. Int. J. Hydrogen Energy 2010, 35 (22), 12660-12667.

25. Wang, H.; Jusys, Z.; Behm, R. J., Electrochemical oxidation kinetics and mechanism of ethylene glycol on a carbon supported Pt catalyst: A quantitative DEMS study. J. Electroanal. Chem. 2006, 595 (1), 23-36.

26. Matos, J. P. F.; Proença, L.; Lopes, M. I. S.; Fonseca, I. T. E., The electrochemical oxidation of xylitol on Pt (111) in acid medium. J. Electroanal. Chem. 2004, 571 (1), 111-117. 27. Liu, J.; Ye, J.; Xu, C.; Jiang, S. P.; Tong, Y., Electro-oxidation of methanol, 1-propanol and 2-propanol on Pt and Pd in alkaline medium. J. Power Sources 2008, 177 (1), 67-70. 
CHAPTER 8 


\section{9 \\ Conclusion and outlook}

Part of this chapter is adapted from:

Ripken, R.M. et al, The hydrogen economy: can APR contribute? In preparation 


\subsection{Conclusion}

In this dissertation, various thermodynamic aspects related to Aqueous-Phase Reforming (APR), as well as various reactor designs have been discussed. Here, process technological aspects have been investigated at the microscale using both theoretical and experimental methods, mainly focusing on the thermodynamics and transport phenomena involved in APR. Four biomass solutions, $10 \mathrm{wt} \%$ of ethylene glycol, glycerol, xylose or xylitol in MilliQ water have been considered throughout this thesis to study these properties and phenomena.

Hydrogen has been identified as an interesting alternative for fossil fuels. Conventionally, hydrogen is produced from natural gas or oil, using high temperature and pressure methods such as steam reforming. In 2002, Aqueous-Phase Reforming was introduced by Cortright et al, to reform oxygenated carbohydrates into hydrogen at more environmental friendly reaction conditions (Chapter 1).

The energy demand of APR was evaluated in terms of the enthalpy and Gibbs free energy of reaction, which were found to be in the same order of magnitude as that of steam reforming (Chapter 2). Importantly, the liquid phase state of water that reacts in the water-gas-shift (WGS) reaction has to be taken into account, while calculating the reaction thermodynamics. In the literature, the WGS is often considered in the vapor phase, whereas our work has demonstrated that water as a reagent is in the liquid phase at APR reaction conditions.

To experimentally study the phase transition of the four biomass model solutions, a high-pressure high-temperature microreactor was developed (Chapter 3). The boiling point of pure water was not significantly affected by the addition of the biomass model substrates. Furthermore, different gas/liquid flow regimes were observed in the microreactor, depending on the gas void fraction. As during APR gas products $\left(\mathrm{H}_{2}, \mathrm{CO}, \mathrm{CO}_{2}, \mathrm{CH}_{4}\right)$ form in a liquid phase, this study provided insights into the possible flow regimes that could occur at low or high gas production rates, such as bubbly flow, Taylor flow and annular flow.

Furthermore, the formation of gas in a liquid phase can affect heat and mass transfer: gaseous products form bubbles on the catalytic surface, blocking the catalyst and therefore preventing further reaction. In addition, the slip length on the bubble/liquid interface is in the same order of magnitude as the microreactor dimensions (microns), and can therefore not be neglected at the microscale. Using a 2D-numerical model in COMSOL Multiphysics 5.3a, we demonstrated that in the presence of bubbles having a hemispherical or elliptical geometry (contact angle $<90^{\circ}$ ), the 
chemical conversion is strongly dependent on the catalytic surface coverage by the bubbles (Chapter 4). As APR is an endothermic reaction, the temperature in the microchannel was found to be directly dependent on the conversion.

However, in hydrophilic catalytic microsystems and when using water as a solvent, bubbles adopt a spherical geometry with large contact angles. Therefore, the pinned bubbles only have a very small contact area with the catalytic surface, compared to the geometry considered in Chapter 4. We hypothesized that transport phenomena could be enhanced in a microreactor with spherical bubbles. To ultimately control the transport phenomena in such a microsystem, bubble nucleation has to be mastered. For this purpose, a microfluidic device was developed with hydrophobic pits, which would act as preferential nucleation sites for the bubbles (Chapter 5). In addition, 3Dnumerical COMSOL simulations demonstrated that the transport phenomena in these microsystems were enhanced, when the catalytic layer was on a different wall than the one on which the bubbles were pinned.

An essential feature in APR microreactors is the solid, immobilized catalyst, which was omitted in the previous chapters. Both a wet chemical, washcoating, and a physical deposition technique, spark ablation, were explored to deposit the catalyst in a microchannel (Chapter 6). Washcoating seemed to be promising to deposit the model catalyst support, $\mathrm{TiO}_{2}$, and a relatively uniform layer could be achieved with good adhesion to the microchannel wall. To deposit the active phase, a physical deposition method was chosen: spark ablation provided high control over the $\mathrm{Cu} / \mathrm{CuO}$ catalyst distribution over the microchannel.

When APR is performed in the microfluidic devices described in this thesis, the outlet stream of the microreactor comprises both a liquid and a gas phase. To obtain the desired hydrogen, or to analyze the gas and liquid phase products individually, these two phases must be separated. For inline analysis, and kinetic measurements in particular, samples must not be averaged over time. For this application, a modular gas/liquid separator based on a micropillar membrane was successfully fabricated, with an estimated dead volume as low as $0.06 \mu \mathrm{L}$ (Chapter 7).

Although APR is already a great improvement over conventional hydrogen production methods in terms of energy demand, it is still dependent on a substantial amount of externally supplied heat. This heat is often supplied by external energy carriers, which are not necessarily sustainable. From this perspective, photocatalytic reforming $(\mathrm{PhCR})$ of biomass, where biomass is reformed into hydrogen and $\mathrm{CO}_{2}$ using only sunlight, is an attractive alternative. The exact reaction mechanism 
and the required catalyst bandgap for PhCR still remain unknown. Cyclic voltammetry in a beaker set-up demonstrated that ethylene glycol and xylitol are likely reformed via adsorption on the catalytic surface, followed by reaction with a hydroxyl radical (Chapter 8). In parallel, first steps towards studying these aspects with a microfluidic device were successfully taken.

\subsection{Outlook}

Currently, APR has been mainly performed in research facilities, whereas the end goal is to implement APR commercially at a large scale, so that hydrogen becomes widely available to all layers of society, from citizens to industry. To the best of my knowledge, Virent Inc., ${ }^{1}$ which was co-founded by Cortright, is the only company using APR commercially as part of a more extended bioreforming process. In the following section, a number of aspects related to the commercialization of hydrogen in general and to that of APR specifically are discussed.

\subsubsection{Biomass feedstock}

As already briefly mentioned in the general introduction (Chapter 1), APR feedstock must be carefully selected to minimize interference with competing food chains. From a sustainability perspective, biomass waste streams would therefore be the best option. This feed, however, consists of a wide range of biomass substrates and includes impurities, such as salts and fatty acids, ${ }^{2-3}$ which may interfere with APR. So far, only a few studies have been conducted on APR of crude glycerol mixtures ${ }^{2-4}$ and biomass polymers. ${ }^{5-6}$ In addition, the ratio of the biomass building blocks (lignin, hemicellulose and cellulose) differs strongly between plant varieties, ${ }^{7-8}$ which requires a robust APR process to cope with these chemical varieties. Therefore, APR must be further developed to process a wider feedstock range of varied composition. Growing dedicated crops with a well-defined composition would be a more feasible sustainable short-term solution. Ideal for this purpose would be fast growing plants, such as algae or water plants. ${ }^{9}$ Not only would a large amount of biomass be produced in a short amount of time, but also the turnover time from $\mathrm{CO}_{2}$ to biomass, from biomass to hydrogen and back to $\mathrm{CO}_{2}$ would be reduced. ${ }^{10}$ 


\subsubsection{Energy source to drive APR}

Next to the biomass feedstock, APR is very much dependent on an external energy source to drive the reaction. Although APR requires less energy than the more conventional steam reforming to produce hydrogen, the enthalpy of reaction for APR model substrates can still be as high as +600 kJ.mole ${ }^{-1}$ substrate. ${ }^{11}$ However, this enthalpy of reaction only refers to the cracking and the watergas-shift reaction, whereas APR of crude biomass can comprise many more steps, such as purification of intermediates. ${ }^{12}$ All these steps must be included in the energy balance.

Some of the required external energy can originate from secondary processes: energy released by exothermic reactions can be utilized to drive endothermic ones. For example, the APR plant could be located next to another chemical production site, or maybe even a power station. Chemical processes that rely on hydrogen, such as ammonia production, would be particularly interesting for this purpose. This organization is beneficial to both APR and the ammonia production: APR utilizes the excess energy from the ammonia production, and the hydrogen is formed close to where it is needed.

Still, recycling of the excess energy produced in other processes does not make APR fully sustainable, and hydrogen renewable. To achieve true sustainability, this additional amount of energy must be provided by renewable energy sources, such as sun light, wind or hydropower. Which renewable energy is the most suitable, depends on the plant location (Chapter 1).

\subsubsection{Production scale: centralized and decentralized production}

A third point to consider when commercializing APR or hydrogen is the production scale. At this moment, it is highly unlikely that APR could provide all the required hydrogen singlehandedly: for APR to contribute significantly to the hydrogen market, to both the chemical and fuel industries, the production scale has to be large (See also section 1.1.2.). Two production scales, or strategies, can be distinguished on how to produce hydrogen: centralized or decentralized production.

Centralized production refers to one facility servicing a large number of clients, maybe even at the national level. The hydrogen would then be produced at a large scale, ${ }^{13}$ and building- and equipment investments shared between the customers. Furthermore, centralized production 


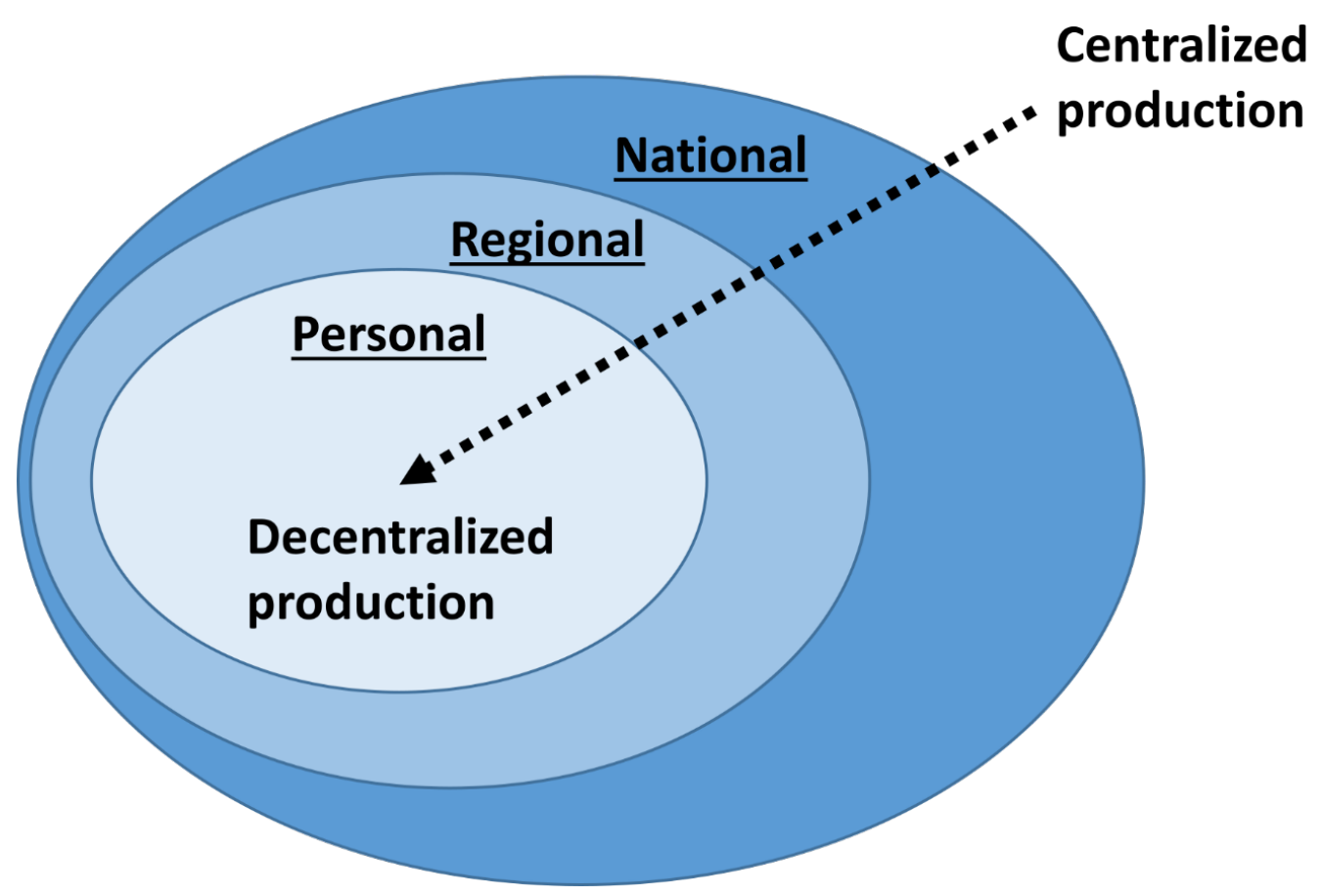

Figure 9.1 Schematic of decentralized compared to centralized levels and their corresponding organizational levels.

benefits from the economy of scale: producing hydrogen in bulk compensates the plant's operational and feedstock costs. ${ }^{14-15}$ The drawback, however, are the increased transportation costs. $^{13,15}$

Production closer to where the product is needed, serving a smaller number of clients on a regional level, results in a higher number of smaller plants. This organization level corresponds to decentralized production. ${ }^{14,16}$ An advantage of this approach is that the production sites can be personalized to the needs of the near-by end-user(s) ${ }^{16}$ such as the biomass feedstock composition or hydrogen purity. However, building multiple specialized smaller plants could lead to higher design and building costs. Some transportation costs are involved in decentralized production at a regional level, but to lesser extent than for centralized hydrogen production. ${ }^{13,15}$

Personal or domestic hydrogen production would be the most extreme form of decentralized production. For example, a car equipped with its own APR unit, producing hydrogen in-situ, which is directly used in a hydrogen fuel cell. The external energy could be supplied by, for example, a solar panel on the roof. Once the process is running, the heat released from the combustion of the hydrogen can be recycled to drive APR. This personalized approach would circumvent the 
customization and/or building of new infrastructure for plant-based hydrogen production altogether.

A highly decentralized approach could become reality by introduction of microfluidic systems developed specifically for domestic APR. However, these microscale devices would not produce large amounts of $\mathrm{H}_{2}$ at a high throughput, even at high reaction yields and reactor efficiency. An average car with a 200 horsepower engine translates to a power around $150 \mathrm{~kW}$, or $150 \mathrm{~kJ} . \mathrm{s}^{-1}$, while the enthalpy of formation $(1 \mathrm{bar} ; 273 \mathrm{~K})$ of water vapor is $-241 \mathrm{~kJ}^{\mathrm{mol}} \mathrm{mol}^{-1}$. So, $0.62 \mathrm{~mol}$ of hydrogen would have to be combusted every second to move the car. It is highly unlikely that, in a microreactor the long chain of consecutive steps (hydrolysis, possible hydrogenation, and APR), can be performed fast enough to supply hydrogen at this rate.

Considering all the above, hydrogen production using APR at the regional level is probably the most viable at this stage: multiple plants, with high production rates, which can still be adapted to the regional requirements, such as type of feedstock. The transportation and infrastructural costs will be higher than for decentralized production at the domestic scale, but still feasible.

\subsubsection{Infrastructure}

The hydrogen transport is another (technological) aspect that has to be addressed before hydrogen can be commercialized. Utilization of existing natural gas pipelines to transport hydrogen would be the most self-evident possibility. This pipeline network and gas connections are already in place, in which compressed nature gas is transported at a pressure between 14 and 103 bar. ${ }^{17}$ The same pipelines would be suitable for compressed hydrogen transport. ${ }^{15}$

When hydrogen is to be used as a fuel, for example, in cars, tank station logistics will require adjustments. First of all, the tank station has to be supplied with hydrogen, either through pipelines, or by fuel vehicles over the road. Trucks would transport hydrogen in dedicated high-pressure tanks, that can withstand the $300-400$ bar pressure that is required to keep the hydrogen in a liquid state. ${ }^{15}$ Furthermore, the tank nozzle will be adapted: conventional nozzles are suitable for liquids at relatively low pressure, not for liquid hydrogen pressurized at hundreds of bar. 


\subsubsection{It all comes down to the hydrogen price}

An important incentive for consumers to choose hydrogen instead of other fuels, would be the hydrogen price. Calculations of the total hydrogen production costs using APR starting from a xylitol syrup were performed by Sladkovskiy et al, ${ }^{18}$ who determined a price of 12.97 dollar per kilo of hydrogen. Almost $92 \%$ of these costs was accounted for by the xylitol syrup feedstock. ${ }^{18}$

However, by using biomass waste streams the feedstock price would be significantly reduced. When the external energy is supplied by using excess energy from other chemical processes, energy costs should also be lowered. Also the use of non-noble metals, such as Ni instead of Pt or Pd for the APR catalyst, would reduce the hydrogen price. ${ }^{19-20}$ As a result, a price of 4 dollar per kilo of hydrogen should be achievable, ${ }^{18}$ so that $\mathrm{H}_{2}$ becomes competitive with the price of crude oil (2.20 dollar per kilo). ${ }^{21}$

The remaining costs for transport and storage are likely more difficult to reduce. Large investments, both corporate and scientific, are required to improve APR/hydrogen technology as well as the infrastructure before hydrogen can be used by a wide range of consumers. National governments, businesses and even global partners would need to collaborate in order to make the hydrogen economy a reality.

\section{References}

1. Virent http://www.virent.com/ (accessed 04-03-2019).

2. Boga, D. A.; Liu, F.; Bruijnincx, P. C. A.; Weckhuysen, B. M., Aqueous-phase reforming of crude glycerol: effect of impurities on hydrogen production. Catalysis Science \& Technology 2016, 6 (1), 134-143.

3. Lehnert, K.; Claus, P., Influence of Pt particle size and support type on the aqueous-phase reforming of glycerol. Catal. Commun. 2008, 9 (15), 2543-2546.

4. Remón, J.; Giménez, J. R.; Valiente, A.; García, L.; Arauzo, J., Production of gaseous and liquid chemicals by aqueous phase reforming of crude glycerol: Influence of operating conditions on the process. Energy Convers. Manage. 2016, 110, 90-112. 
5. Zhang, J.; Yan, W.; An, Z.; Song, H.; He, J., Interface-Promoted Dehydrogenation and Water-Gas Shift toward High-Efficient H2 Production from Aqueous Phase Reforming of Cellulose. ACS Sustainable Chemistry \& Engineering 2018, 6 (6), 7313-7324.

6. Wen, G.; Xu, Y.; Liu, Q.; Wang, C.; Liu, H.; Tian, Z., Preparation of Ce-modified Raney Ni Catalysts and Their Application in Aqueous-Phase Reforming of Cellulose. Catal. Lett. 2011, 141 (12), 1851-1858.

7. Vassilev, S. V.; Baxter, D.; Andersen, L. K.; Vassileva, C. G., An overview of the chemical composition of biomass. Fuel 2010, 89 (5), 913-933.

8. Huber, G. W.; Dumesic, J. A., An overview of aqueous-phase catalytic processes for production of hydrogen and alkanes in a biorefinery. Catal. Today 2006, 111 (1), 119-132.

9. Cheng, X.; Ooms, M. D.; Sinton, D., Biomass-to-biocrude on a chip via hydrothermal liquefaction of algae. Lab on a Chip 2016, 16 (2), 256-260.

10. Martens, J. A.; Bogaerts, A.; De Kimpe, N.; Jacobs, P. A.; Marin, G. B.; Rabaey, K.; Saeys, M.; Verhelst, S., The Chemical Route to a Carbon Dioxide Neutral World. Chemsuschem 2017, $10(6), 1039-1055$.

11. Ripken, R. M.; Meuldijk, J.; Gardeniers, J. G. E.; Le Gac, S., Influence of the Water Phase State on the Thermodynamics of Aqueous-Phase Reforming for Hydrogen Production. Chemsuschem 2017, 10 (24), 4909-4913.

12. Melin, K.; Kohl, T.; Koskinen, J.; Hurme, M., Performance of biofuel processes utilising separate lignin and carbohydrate processing. Bioresour. Technol. 2015, 192, 397-409.

13. Levin, D. B.; Chahine, R., Challenges for renewable hydrogen production from biomass. Int. J. Hydrogen Energy 2010, 35 (10), 4962-4969.

14. Balat, M., Potential importance of hydrogen as a future solution to environmental and transportation problems. Int. J. Hydrogen Energy 2008, 33 (15), 4013-4029.

15. Ferreira-Aparicio, P.; Benito, M. J.; Sanz, J. L., New Trends in Reforming Technologies: from Hydrogen Industrial Plants to Multifuel Microreformers. Catalysis Reviews 2005, 47 (4), 491-588.

16. Prince-Richard, S.; Whale, M.; Djilali, N., A techno-economic analysis of decentralized electrolytic hydrogen production for fuel cell vehicles. Int. J. Hydrogen Energy 2005, 30 (11), 1159-1179.

17. NatGas The Transportation of Natural Gas. http://naturalgas.org/naturalgas/transport/. 
18. Sladkovskiy, D. A.; Godina, L. I.; Semikin, K. V.; Sladkovskaya, E. V.; Smirnova, D. A.; Murzin, D. Y., Process design and techno-economical analysis of hydrogen production by aqueous phase reforming of sorbitol. Chemical Engineering Research \& Design 2018, 134, 104-116.

19. Holladay, J. D.; Hu, J.; King, D. L.; Wang, Y., An overview of hydrogen production technologies. Catal. Today 2009, 139 (4), 244-260.

20. Chen, G.-y.; Li, W.-q.; Chen, H.; Yan, B.-b., Progress in the aqueous-phase reforming of different biomass-derived alcohols for hydrogen production. Journal of Zhejiang UniversitySCIENCE A 2015, 16 (6), 491-506.

21. OilPrice OilPrice https://oilprice.com/ (accessed 25-03-2019). 


\section{Samenvatting}

Klimaatverandering, wat zeer waarschijnlijk veroorzaakt door menselijke activiteit, is één van de belangrijkste uitdagingen waar de wereldbevolking nu mee te maken heeft. De uitstoot van $\mathrm{CO}_{2}$ en andere broeikasgassen, die vaak worden gevormd tijdens de verbranding van fossiele brandstoffen, moet drastisch worden ingeperkt om daarmee verdere temperatuurstijging op aarde en alle ernstige gevolgen daarvan te voorkomen. Om dit te bereiken, zal de wereld van fossiele brandstoffen over moeten gaan op duurzame alternatieven.

Waterstof is veelbelovend als vervanging voor fossiele brandstoffen. Echter, waterstof wordt nog altijd voornamelijk geproduceerd uit uitputtende en onduurzame bronnen, zoals gas en kool, door middel van processen die plaatsvinden op hoge temperatuur en druk. In dit proefschrift is het omzetten van biomassa dat is opgelost in een vloeibare waterfase (Aqueous-Phase Reforming, APR) onderzocht als mogelijke route voor waterstofproductie. Met name de fysisch-chemische en proces-technologische aspecten van dit proces zijn bekeken. Tijdens APR worden zuurstofrijke koolwaterstoffen, zoals suikers en polyalcoholen, omgevormd tot waterstof op relatief lage temperatuur $(<550 \mathrm{~K})$ en druk $(<55 \mathrm{bar})$. Een algemene introductie tot dit onderwerp is gegeven in Hoofdstuk 1.

De benodigde energie voor de omzetting van vier biomassa modelverbindingen, 10 gewichtsprocent ethyleen glycol, glycerol, xylose en xylitol in MilliQ water, zijn berekend in Hoofdstuk 2, in termen van de enthalpie en de Gibbs vrije energie. Aangezien het hierbij van belang is dat de juiste aggregatietoestand van de reactanten in acht wordt genomen, is eerst de fasetoestand van deze modeloplossingen bepaald. Water dat reageert in APR wordt in de literatuur in de gasfase beschouwd, terwijl resultaten in dit proefschrift laten zien dat juist een vloeistoffase moet worden gebruikt in thermodynamische berekeningen. De energieberekening met de juiste, vloeibare fasetoestand van de oplossingen toonde aan dat de omzetting in vloeibare fase vergelijkbaar is met die van de omzetting in de gasfase, in tegenstelling tot wat er is beschreven in de literatuur.

In Hoofdstuk 3 is de ontwikkeling van een hoge druk en hoge temperatuur microfluïdische opstelling beschreven om de faseovergangen ook experimenteel te bepalen. De concentratie van de model biomassacomponenten was dermate laag dat de faseovergangen vergelijkbaar waren met 
die van puur water. Daarbij is ook gekeken naar het kookmechanisme (explosief koken in tegenstelling tot het koken vanuit een kernpunt) en de daaropvolgende gas/vloeistof stromingsregimes. Deze stromingspatronen dienden als model voor de stromingspatronen die kunnen ontstaan als gevolg van de vorming van waterstofgas en -bellen tijdens APR.

De vorming en aanwezigheid van zulke bellen in de vloeistoffase hebben een negatief effect op het warmte- en massatransport in een microreactor, des te meer wanneer deze bellen zich vormen op het katalytisch oppervlak. In Hoofdstuk 4 zijn zowel het moment van de vloeistof als de transportverschijnselen numeriek bestudeerd voor een 2D-microreactor door middel van COMSOL Multiphysics 5.3a simulaties. Specifiek is er gekeken naar bellen die een contacthoek kleiner dan $90^{\circ}$ hebben met het katalytisch oppervlak. Zowel de conversie als het warmtetransport verliep minder effectief als gevolg van het grote contactoppervlak van deze bellen met de katalysator, in vergelijking tot een situatie zonder bellen.

In realistische, hydrofiele katalystische microsystemen waarin water wordt gebruikt als oplosmiddel, vormen echter bellen met een grotere contacthoek. Hierdoor is het contactoppervlak van de bel met de katalytische laag kleiner in vergelijking tot het contactoppervlak van bellen met een kleinere contacthoek. In Hoofdstuk $\mathbf{5}$ is numeriek aangetoond dat deze bolvormige bellen niet langer het katalytische oppervlak en de reactie blokkeren. Om uiteindelijk de transportverschijnselen te kunnen beïnvloeden, zal de belvorming moeten plaatsvinden onder gecontroleerde omstandigheden. In hetzelfde hoofdstuk is een microreactor beschreven met hydrofobe nucleatieplaatsen voor dit specifieke doel. Daarnaast toonden de numerieke simulaties in hoofdstuk 5 aan dat het massatransport toeneemt wanneer het katalytisch oppervlak zich op een andere wand bevindt dan de wand waar de bellen worden gevormd.

Om waterstofbellen te kunnen generen, moet een heterogene katalysator worden geïntroduceerd in de APR microreactor. In Hoofdstuk 6 zijn zowel een nat chemische als een fysische techniek beschreven om een katalysator lokaal in een microkanaal te deponeren: "washcoating" is gebruikt om een $\mathrm{TiO}_{2}$ basislaag te deponeren, terwijl een techniek gebaseerd op ablatie ten gevolge van elektrische vonken is onderzocht voor de depositie van de katalytisch actieve $\mathrm{Cu}$-component.

Het analyseren van zowel de gasvormige als vloeibare producten van APR vergt het scheiden van deze twee fasen. In Hoofdstuk 7 is een gas/vloeistof module ontwikkeld voor dit doeleinde. Deze module is gebaseerd op een membraan bestaande uit micropillaren en heeft een geschat dood 
volume van $0.06 \mu \mathrm{L}$. Deze module kan worden gebruikt voor vloeistofsnelheden tot $1000 \mu \mathrm{L} . \mathrm{min}^{-}$ ${ }^{1}$ en is ideaal voor de directe koppeling met analyseapparatuur.

Hoewel de omzetting in vloeibare fase minder energie vergt in vergelijking tot omzetting in de gasfase, wordt er nog steeds een substantiële hoeveelheid energie verbruikt door de APR-reactor. Deze energie komt van externe bronnen, die niet per definitie duurzaam is opgewekt. Fotokatalytische omzetting (Photocatalytic Reforming, PhCR) is onderzocht als alternatief proces in Hoofdstuk 8, waarbij enkel zonlicht wordt gebruikt als externe energiebron. In het bijzonder is gekeken naar de fotokatalytische activiteit van de vier bovengenoemde biomassa model substraten en hun reactiemechanismen. Verder is er een nieuwe microreactor gepresenteerd om de elektrochemische eigenschappen van de reactie te bestuderen, waarbij de gas- en vloeistofstromen gescheiden worden in diezelfde reactor.

Tot slot zijn de belangrijkste conclusies en de mogelijke commercialisering van het omzetting van biomassa opgelost in een vloeibare waterfase besproken in Hoofdstuk 9. Onder andere de grondstoffen, de energiebronnen en de productieschaal komen daar aan bod. 


\section{Acknowledgements}

Over four years ago, I moved to Enschede to start my PhD-life. Little did I know what was waiting for me... Now, looking back, I would describe this period one of the toughest, but, more importantly, also as one of the most satisfactory. Trained as a chemist, I had to learn so much about engineering and that learning curve was steep at times. I would like to thank all those people that helped me along this way, as I now dare to call myself a chemist, but with some engineering expertise.

First of all, merci Séverine, for giving me the opportunity to make the transition from chemistry to microscale engineering and helping me to color in my PhD-project. Literally, in some cases: I have most certainly discovered a new passion for yellow! Also a big thank you for correcting all those pieces of writing and lifting my papers to a new level. Thanks for all the good discussions, including those about art or dance, and all the coffees we shared.

I would also like to thank my promotor and other supervisor, Han, voor al het advies wanneer ik weer eens vast kwam te zitten, het vertrouwen in mij en de vrijheid die je me hebt gegeven om mijn eigen interesses te volgen deze vier jaar.

Two chapters in this thesis are fully based on the work of my bachelor student Constantijn Rip and my master student Vincent de Boer, who were brave enough to join me on my journey. Constantijn, het concept voor de gas/vloeistof microseparator ligt er nu en ik heb goede hoop dat dit verder wordt ontwikkeld. Tot op vandaag heb ik nog altijd geen bedrijf gevonden die deze module verkoopt met een dergelijk klein dood volume als dat jij hebt weten te bereiken. Vincent, bedankt voor jouw eindeloze creativiteit en zelfstandigheid. Ondanks dat het geen makkelijk project was, heb jij meerdere microreactoren ontwikkeld voor de fotokatalytische omzetting van biomassa, waar naar mijn mening de toekomst ligt voor waterstofproductie uit biomassa. Furthermore, Aura and Erilia, I enjoyed our collaboration on the catalyst deposition techniques: Chapter 6 includes some promising results which could help chemists and engineers to design and fabricate catalytic microreactors.

Jeff, thank you for your valuable contribution to Chapters 4 and 5. The discussions with you have not only taught me so much about transport and interfacial phenomena, but also on how to correctly simulate these properties. Thank you for your patience with me and the collaboration! 
During these four years I had the opportunity to work in a multidisciplinary and international team, not only within the MCEC consortium, but also at the University of Twente. For me, the scientific discussions, but also the informal contact has not only taught me a lot of science, but also gave me the best support and friends that I could I have wished for. Hai, nobody dances the Macarena like you do! Miguel, Aura, Vasilis, Corentin, Christina, Jean-Baptiste, and Bastien, thank you so much for all the great lunch breaks and the fun we had! I will miss you a lot!

The multidisciplinarity of my project also led me to several UT-laboratories. First of all, the combined IDS/MCS lab. Remco, ik ben bijzonder blij dat je me wilde helpen met de elektronische componenten van mijn set-up, zonder jou had ik ongetwijfeld een keer de stroom doen uitvallen. I would also like to thank Jarno, Dennis, Henk-Willem, Marcel and Dirk for sharing the lab space with me and tolerating my choice of music and incidentally my noises of frustration. Also a big thank you to the MCS group members, for the nice chats in the hallways and many many good cakes.

Another space at the UT invaluable for my PhD was the Nanolab cleanroom, where all my microfluidic devices were fabricated. Stefan, thank you for all those hours in the cleanroom and brainstorming with me about the chip design. I learned a lot, and I enjoyed working together.

Not only did I work in many laboratories, I also I changed offices 5 times over the years. Luckily, I had my office mates who moved with me. Thank you Adithya and Yawar for introducing me to AMBER and the UT. Carlo, your arrival was a fresh Italian wind in a mainly French office! Bastien and Jean-Baptiste, mes amis français. Sacré bleu, I had some much fun with you! Jean-Baptiste, merci for scientifically growing up with me these four year. Thank you for sharing your knowledge with me and teaching me that there is a definite difference between brie and camembert. Also, I won't forget our experiments on dry-ice bubbles. We still have work to do on the coloring though... I am happy that you agreed to be my paranymph.

I even got the chance to spend some time abroad and in 2018, I left Twente for the Guenther Lab in Toronto, Canada for 4.5 months. Axel, thank you for giving me this chance, and to fulfill my long-term dream to do research abroad. Shashi, Wuyang, Brady, Kelvin, Nima and Dan, thank you so much for welcoming me, I still look back at the lunches under what we started to call 'our tree', in company of the cheeky birds in love with Greek food. Thanks for showing me the real Toronto and the great restaurants, I never knew I could eat so much sushi.... 
Regardless of my whereabouts, the connection with my "studybuddies" from Nijmegen stayed strong. Bart, Elles, Ton, Joske en Wouter, bedankt voor alle die mooie momenten, spelletjes, en goede slechte grappen. Joske, daarnaast wil ik jou ook bedanken dat je aan mijn zijde wil staan tijdens mijn verdediging. Het is fijn om iemand die mij zo goed kent naast me te mogen hebben staan.

I am so lucky to always have had the support of my family. Mam, pap, Chris en Lise, zo ontzettend fijn om thuis te kunnen komen bij jullie en op de bank te kunnen ploffen, Syra op schoot en Stapper aan mijn voeten. Jullie onvoorwaardelijke steun en het kunnen delen van mijn mijlpalen hebben mijn promotietijd zoveel mooier gemaakt.

Lieve Bart, of we nu naast elkaar op de bank zaten of dat er dat zo'n kleine $6000 \mathrm{~km}$ tussen zat, je was en bent er altijd voor me. "Komt goed" zei je altijd als het even tegen zat en dat is het ook gekomen. Heel erg bedankt voor telkens dat laatste zetje, zo fijn om alles met je te kunnen delen. Zo is elke reis is bijzonder en ik heb ontzettend veel zin om met jou verder de wereld te gaan ontdekken.

Liefs, Renée

May, 2019 


\section{About the author}

Renée Maria Ripken was born on the $14^{\text {th }}$ of January 1991, in Waalwijk, The Netherlands. In 2009, she graduated from Het Stedelijk Gynasium van 's Hertogenbosch, after which she started her Bachelor Chemistry at the Radboud University in Nijmegen. She obtained her Bachelor degree in 2012, studying Grignard reaction intermediates in a microfluidic system using inline FTIR analysis during her graduation project.

Renée continued with a Master at the same university, where she was involved in the UltraSense NMR program in the group of Prof. Floris Rutjes and Dr. Martin Feiters. During her Master project, she developed a gas/liquid

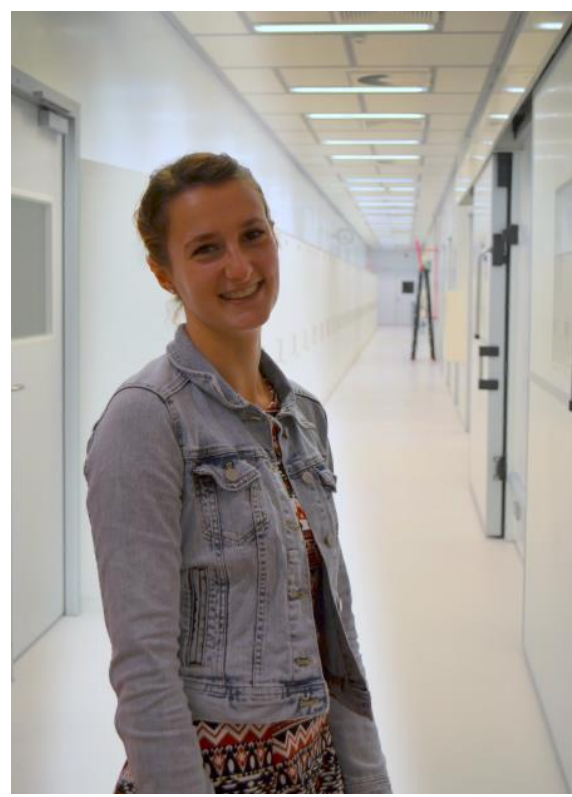
microfluidic system integrated with a stripline NMR to hyperpolarize biomarkers for early medical diagnostics. This project was a collaboration between the Radboud University Nijmegen and FutureChemistry B.V. Renée came first into contact with process technology during a short internship at Eindhoven University of Technology in the group of Prof. Jaap Schouten. There she worked on the modeling of the Spinning Disc Reactor. In 2015, she obtained her Master degree bene meritum.

In the same year Renée started as a PhD-student in the Applied Microfluidics for BioEngineering group and the Mesoscale Chemical Systems group under the supervision of Prof. Dr. Ir. Séverine Le Gac and Prof. Dr. Han Gardeniers at the University of Twente. Her project was part of the Multiscale Catalysis for Energy Conversion (MCEC) consortium, a collaboration between the University of Twente, Utrecht University and Eindhoven University of Technology. During her 4year PhD, Renée studied Aqueous-Phase Reforming of biomass for renewable hydrogen at the microscale. The thermodynamics and process technological aspects were investigated using both theoretical and experimental methods. In the same period, she gained international experience in the group of Dr. Axel Guenther at the University of Toronto, Canada during 4.5 months. Renée will publicly defend her PhD-thesis on June $28^{\text {th }}, 2019$ in Enschede, The Netherlands. 


\section{Scientific output}

\section{List of publications}

Ripken, R. M.; Wood, J.A.; Gardeniers, J. G. E.; Le Gac, S., Aqueous-Phase Reforming in a microreactor: the role of surface bubbles Submitted 2019

Ripken, R. M.; Schlautmann, S.; Sanders, R. G. P.; Gardeniers, J. G. E.; Le Gac, S., Monitoring phase transition of aqueous biomass model substrates by high-pressure and hightemperature microfluidics. ELECTROPHORESIS 2019, 40 (4), 563-570

DOI: $10.1002 /$ elps.201800431

Ripken, R. M.; Wood, J.A.; Schlautmann, S.; Guenther, A.; Gardeniers, J. G. E.; Le Gac, S., Controlled bubble nucleation in gas-liquid-solid catalytic microsystems for enhanced mass transport MicroTAS, Proceedings 2018

Ripken, R. M.; Gardeniers, J. G. E.; Le Gac, S., Real-time monitoring of complex multiphase behavior in a high pressure and high temperature microfluidic chip. MicroTAS, Proceedings 2017

Ripken, R. M.; Meuldijk, J.; Gardeniers, J. G. E.; Le Gac, S., Influence of the Water Phase State on the Thermodynamics of Aqueous-Phase Reforming for Hydrogen Production. Chemsuschem 2017, 10 (24), 4909-4913

DOI: $10.1002 /$ cssc. 201700189

van Gool, J. J. F.; van den Broek, S. A. M. W.; Ripken, R. M.; Nieuwland, P. J.; Koch, K.; Rutjes, F. P. J. T., Highly Controlled Gas/Liquid Processes in a Continuous Lab-Scale Device. Chemical Engineering \& Technology 2013, 36 (6), 1042-1046

DOI: $10.1002 /$ ceat.201200553 


\section{Conference contributions}

Oral presentations

Ripken, R.M.; Wood, J.A.; Gardeniers, J.G.E.; Le Gac, S.

Influence of bubble growth at the catalytic surface on heat and mass transfer in gas-liquidsolid microreactors

$15^{\text {th }}$ International Conference on Micro Reaction Technology (IMRET)

21-24 October, 2018, Karlsruhe, Germany

Ripken, R.M.; Wood, J.A.; Gardeniers, J.G.E.; Le Gac, S.

Influence of bubble growth at the catalytic surface on the heat and mass transfer in gasliquid-solid microsystems

Annual Meeting American Institute for Chemical Engineers (AIChE)

29 October - 3 November, 2017, Minneapolis, USA

Ripken, R.M.; Gardeniers, J.G.E.; Le Gac, S.

Real-Time Monitoring of Complex Multiphase Behavior in a High Pressure High Temperature Microfluidic Chip

Annual Meeting American Institute for Chemical Engineers (AIChE)

29 October - 3 November, 2017, Minneapolis, USA

\section{Poster presentations}

Ripken, R.M.; Wood, J.A.; Schlautmann, S.; Guenther, A.; Gardeniers, J.G.E.; Le Gac, S.

Controlled bubble nucleation in gas-liquid-solid catalytic microsystems for enhanced mass transport

\section{MicroTAS}

11-15 November, 2018, Kaohsiung, Taiwan

Ripken, R.M.; de Boer, V.J.H.W,.; Gardeniers, J.G.E.; Le Gac, S.

Photocatalytic reforming of biomass for hydrogen production

Annual Meeting American Institute for Chemical Engineers (AIChE)

29 October to 3 November, 2017, Minneapolis, USA 
Ripken, R.M.; Gardeniers, J.G.E.; Le Gac, S.

Real-Time Monitoring of Complex Multiphase Behavior in a High Pressure High Temperature Microfluidic Chip

MicroTAS

22-26 October, 2017, Savannah, USA

de Boer, V.J.H.W.; Ripken, R.M.; Gardeniers, J.G.E.; Le Gac, S.

Photocatalytic reforming of biomass for hydrogen production

Netherlands Chemistry and Catalysis Conference (NCCC)

6-8 March, 2017, Noordwijkerhout, The Netherlands

Ripken, R.M.; Gardeniers, J.G.E.; Le Gac, S.

Thermodynamic study of aqueous phase reforming for hydrogen production

Netherlands Chemistry and Catalysis Conference (NCCC)

7-9 March 2016, Noordwijkerhout, The Netherlands

Ripken, R.M.; van Weerdenburg, B.J.A.; Tijssen, K,; Burgers, R.M.; van den Broek, S.A.M.W.; Nieuwland, P.J.; van Bentum, P.J.M.; Kentgens, A.P.M.; Feiters, M.C.; Rutjes, F.P.J.T.

In-Line Monitoring of Continuous Gas-Liquid Reaction by NMR

Netherlands Chemistry and Catalysis Conference (NCCC)

10-12 March, 2014, Noordwijkerhout, The Netherlands

\section{Awards}

\section{Ripken, R.M}

Second place at the AIChE 2017 Sustainability Poster session Photocatalytic reforming of biomass for hydrogen production Annual Meeting American Institute for Chemical Engineers (AIChE)

29 October - 3 November, 2017, Minneapolis, USA 
